\title{
THE USE OF DIGITAL TECHNOLOGIES \\ IN AN ENGLISH-MEDIUM INSTRUCTION CONTEXT: \\ A case study of Vietnamese higher education teachers and students
}

BY

VO DOAN THO

\begin{abstract}
A thesis
submitted to the Victoria University of Wellington

in fulfilment of the requirements for the degree of

Doctor of Philosophy
\end{abstract}

VICTORIA UNIVERSITY OF WELLINGTON 


\section{Abstract}

English-medium instruction (EMI) is a global trend in higher education which coincides with the digital age. This thesis examines the uses of digital technologies in an EMI context in Vietnamese higher education. It explores how teachers and students used digital technologies and how they perceived the development of students' learning through digital technologies in the EMI environment.

The methodological approach taken was a qualitative multiple case study underpinned by an interpretive paradigm. Each case included one subject teacher and a class of 40 to 50 students in an undergraduate economics-related courses taught in English. Data from the four cases were collected during the first year of EMI implementation, from August to December 2017 from three sources of information: teacher semi-structured interviews, classroom observations, and student focus-group discussions. The data were abductively analysed following the process of constructing themes suggested by Vaismoradi et al. (2016) and adapted from the thematic analysis method of Braun and Clarke (2012).

Within this context, the teachers and students used a range of digital technologies for teaching and learning activities. The technologies included digital devices (e.g. computers, laptops, smartphones, and tablets), search engines (Google, and Wikipedia), presentation tools (PowerPoint, and Prezi), organisation tools (Google drive, and Dropbox), social networks (YouTube, and Facebook), and the learning management system (LMS). The teachers used technology to address challenges they faced through EMI teaching. Their practice with technology included curating and developing materials with digital resources, presenting subject matter with multimedia and organising classes with cloud storage and the LMS for uploading materials or communicating with the students. They believed that using technology improved their students' understanding of content knowledge, learning of English vocabulary, engagement and motivation. The students expressed confidence in using digital technologies for learning within and beyond the classroom. They reported deploying technology to search for materials, upload and download information and resources, and to organise lesson content. They proactively used technology to personalise their learning by accessing informal online activities and engaging with collective learning networks, which enabled them to collaborate and gain support for learning. The students believed that digital technologies played an integral part in enhancing their understanding of subject matter and improving their English vocabulary and skills.

Teachers and students became agentic as they adapted to the new EMI context. The teachers endeavoured to adjust their teaching in response to changes including the neoliberal 
system in $\mathrm{HE}$, the rapid technological development and practices demanded by the change of instruction language. Access to digital resources appeared to enable them to independently make pedagogical decisions and take a proactive role in EMI programmes. However, there were few substantive changes in pedagogical practice. Different influences which possibly reduced the teachers' professional agency in completely changing pedagogy with technology included their technological, content, and pedagogical knowledge and beliefs, or conflicting influences from Confucian educational practices, belief in a teacher-centred and content-driven approach, and the exam-oriented system. The students had a strong sense of agency as proactive learners in the digital age. They were autonomous in their learning with innovative uses of technology in the EMI environment. Those uses of technology offered them collective support and facilitated them to independently cope with many changes in the EMI learning context. This raises some implications not only for institutional policy for professional development which encourages teachers' collaboration but also for the learning support scheme and teaching practices which offer students opportunities to access collaborative support and tasks.

The ROAD-MAPPING framework (Dafouz \& Smit, 2020) shed light on the multifaceted nature of EMI programmes in the Vietnamese context. It highlighted the impact of glocalisation in shaping EMI policies in Vietnamese HE institutions. The introduction of EMI at the participating university was the policy makers' response to internationalisation where global academic programmes were imported into this local context. A number of contextual factors influenced the process of EMI implementation such as the predominant role of Vietnamese as language of instruction in most academic programmes, the lack of focus on English development and requirement in EMI curriculum and language policy, the textbook-based system, and inadequate preparation for both subject teachers and students. These characteristics in the Vietnamese education context shaped EMI teaching practices in which the teachers and students focussed on disciplinary knowledge and expected English skills to follow. This suggests the synergy of 'global' and 'local' factors needs careful attention if EMI is to work in practice. 


\section{Acknowledgements}

I would like to take this opportunity to acknowledge the following people for their valuable contributions to the writing and completion of this thesis.

My deep appreciation and respect to my supervisors, Dr. Louise Starkey and Dr. Margaret Gleeson, for their endless support, guidance, and encouragement. They facilitated me during the entire process with their practical advice, constructive feedback, and dedicated supervision. Thank you to the teacher and student participants for their willingness to share their experiences and let me into their thoughts and their classrooms. They contributed their input, time and effort to make this study possible. I would like to express my gratitude to the principal of the university, and the heads of schools for giving me permission and supporting me to conduct my research.

Special thanks go to the Vietnamese Government and Victoria University of Wellington, who granted me the scholarship enabling the study. To the School of Education and the Wellington Faculty of Graduate Research (VUW), who provided me with great support during my study. To my university and my school in Vietnam, who motivated me to pursue my goals. To Argo Trust managers, staff and residents, who inspired me with their values in life.

I am also grateful to Dr. Kabini Sanga, who gave me helpful guidance in qualitative research, and to the learning advisors, especially Dr. Deborah Laurs and Kirsten Reid, for giving me invaluable advice on academic writing and speaking. I would like to thank Dr. Tien Ho and Dr. Le Nguyen for the reference they provided to support my study application. Sincere thanks go to Dr. Shelley Dawson and Dr. Luke Santamaria, for helping me with the proofreading of my thesis. I thank all of my PhD friends and officemates, but give special mention to Nga Ngo and Rana Daoud, for their sharing of understandings and advice to me. Thank you to my best friends and colleagues in Vietnam for their encouragement. I must acknowledge Adrienne and Leon for being kind and supportive during my stay at their house.

My family has always been by my side during my academic journey. I would like to acknowledge their unconditional love and support. In particular, I would like to thank my parents, my in-laws, and my mom, to whom I own a debt of gratitude. Thank you to my siblings, my nieces, and my nephews, who keep communicating with me and encouraging me in my hard time. Finally, my heartfelt thanks go to my dear wife, Thu Dang, who always believes in my ability, offers me care, understanding and acceptance, and shares with me everything. Thank you all for always being there. 


\section{Contents}

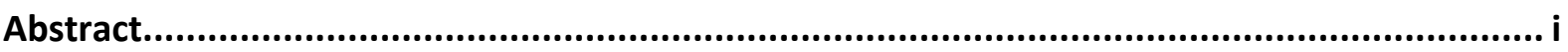

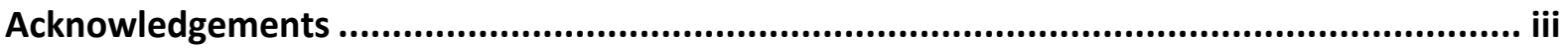

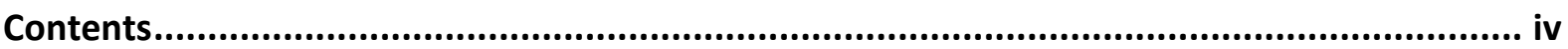

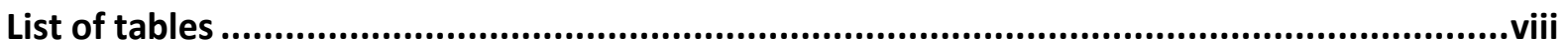

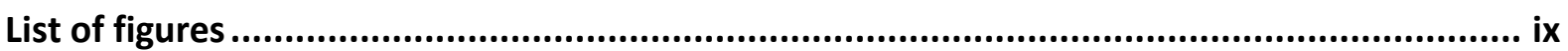

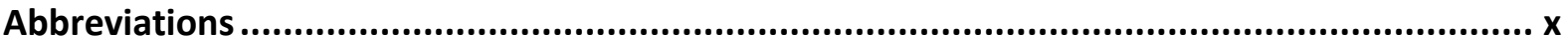

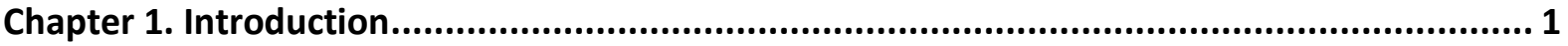

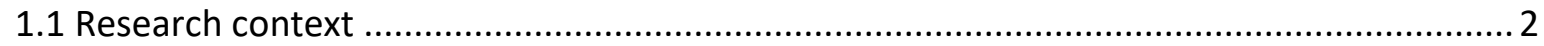

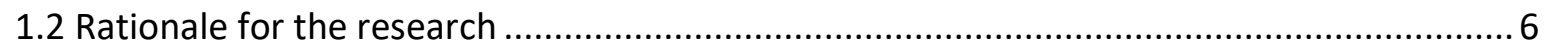

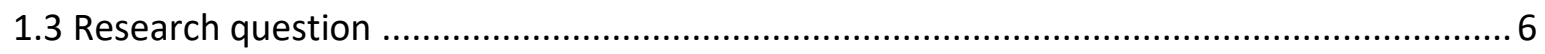

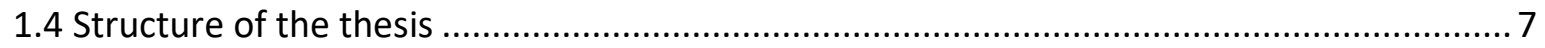

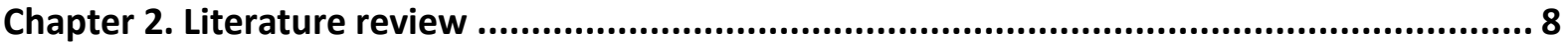

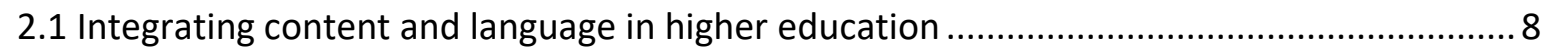

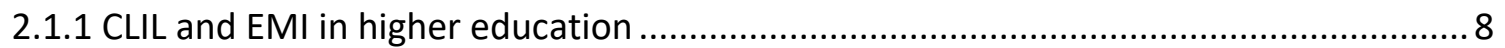

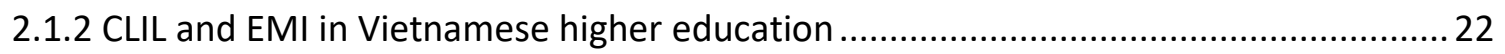

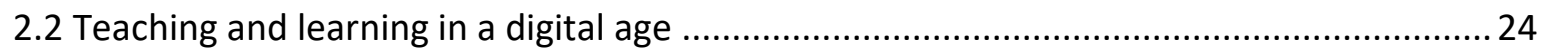

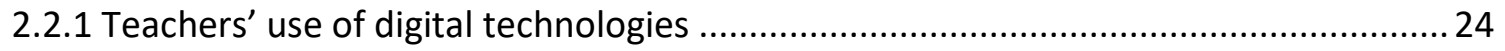

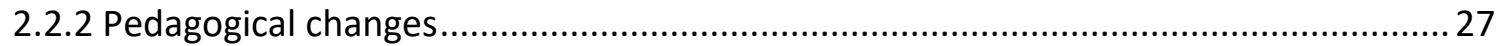

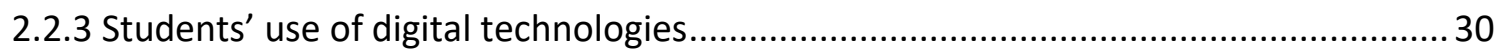

2.2.4 Factors influencing the integration of digital technologies........................................ 31

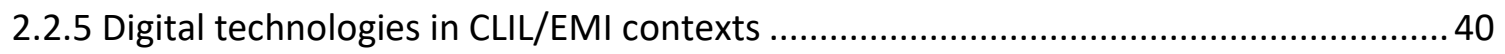

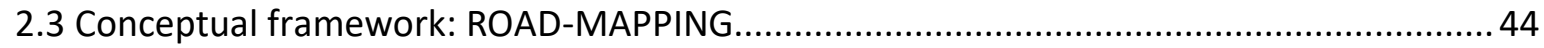

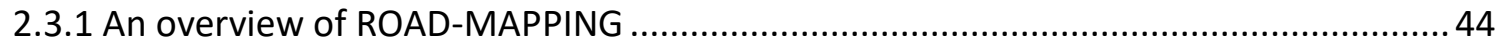

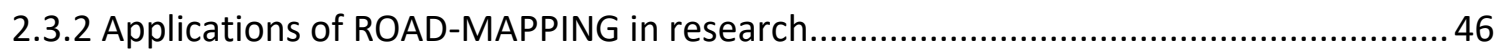

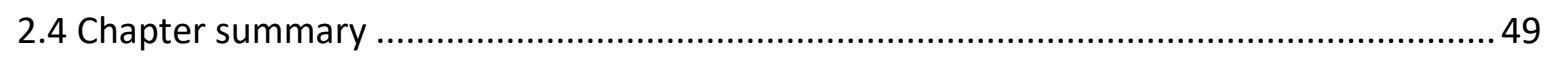

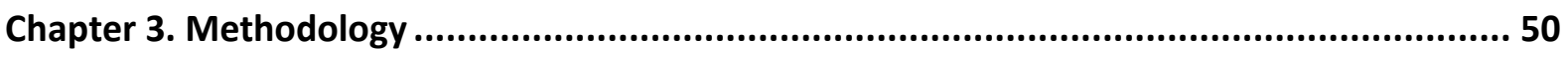

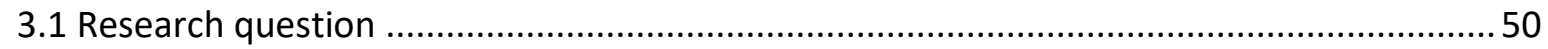

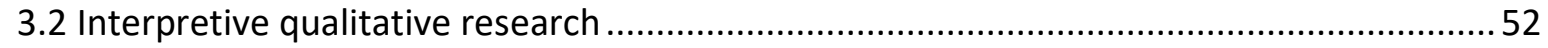

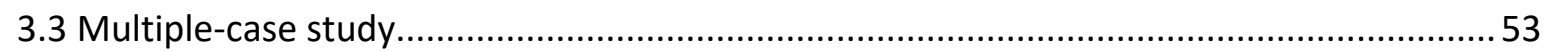

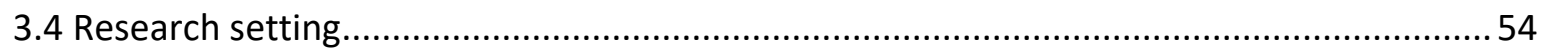

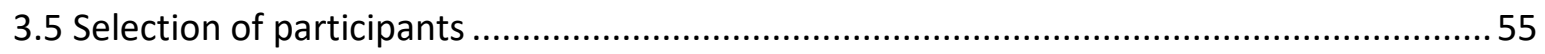

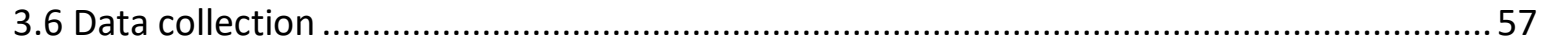




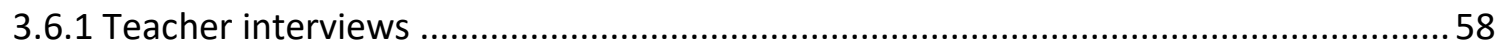

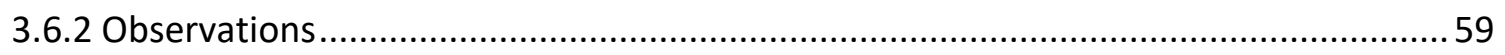

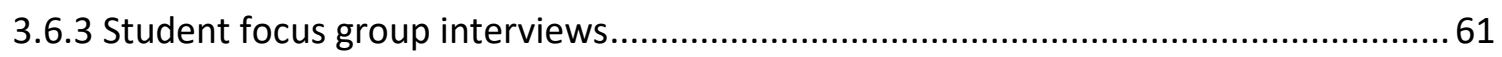

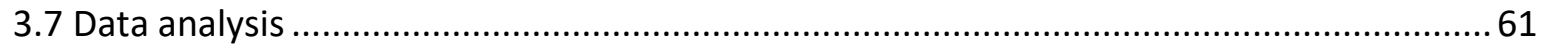

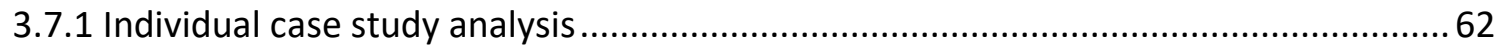

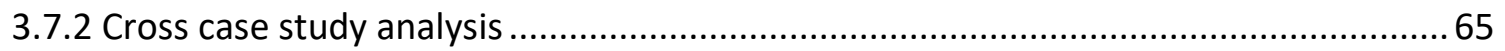

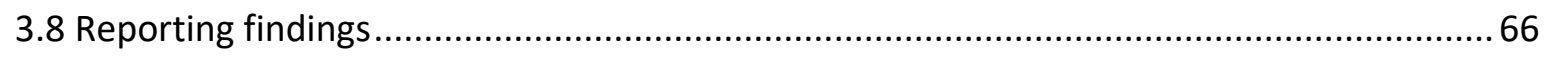

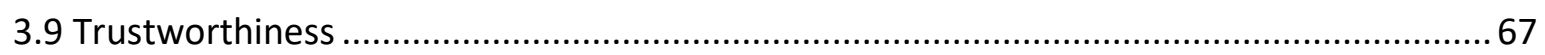

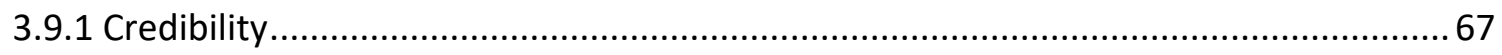

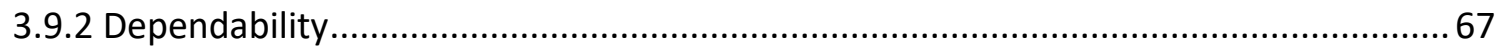

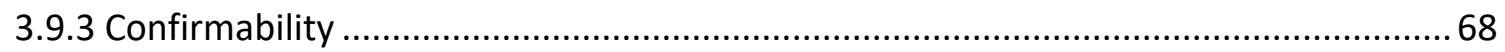

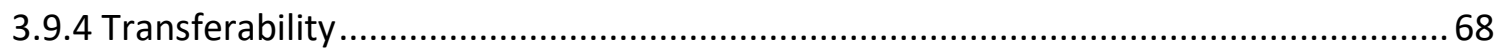

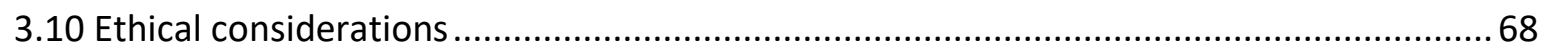

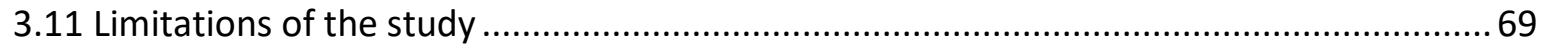

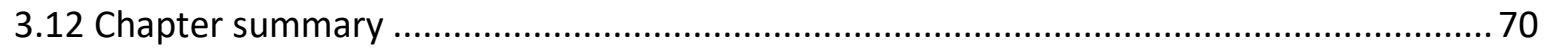

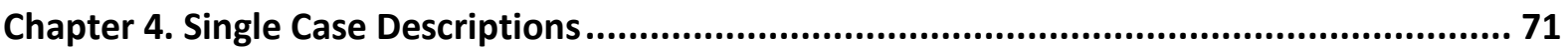

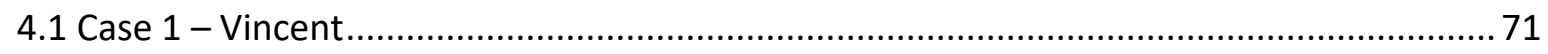

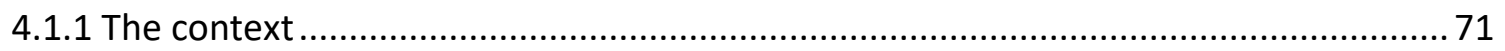

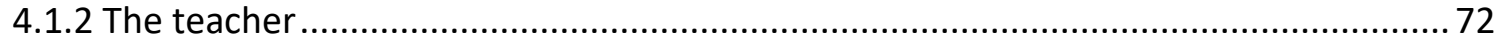

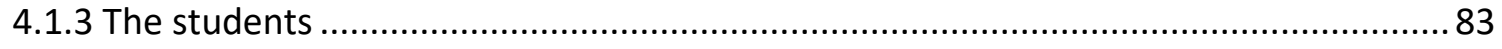

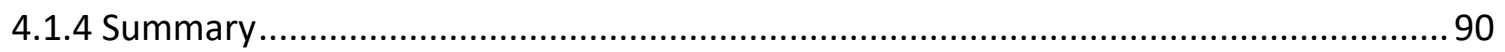

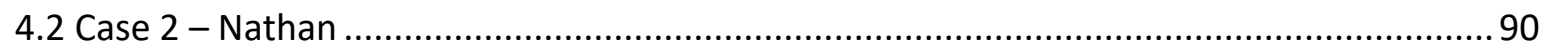

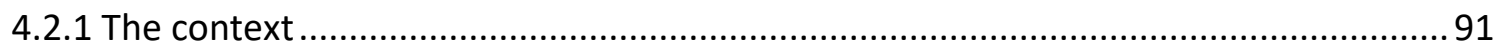

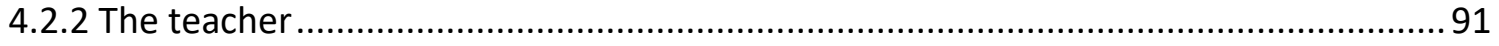

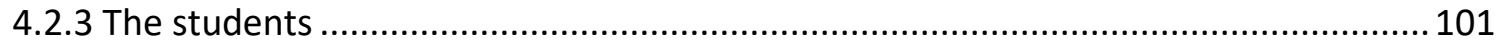

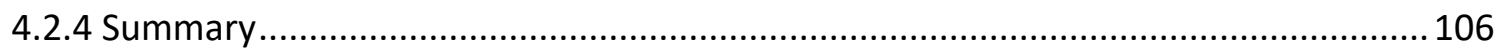

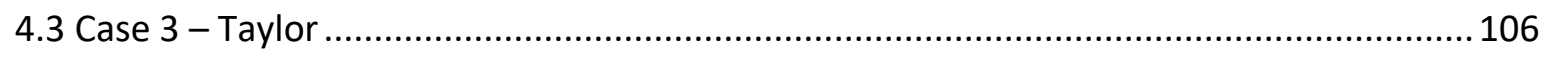

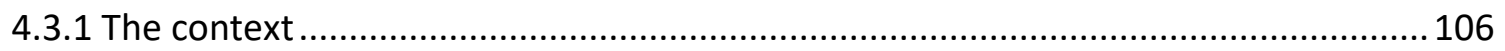

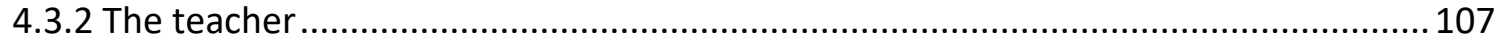

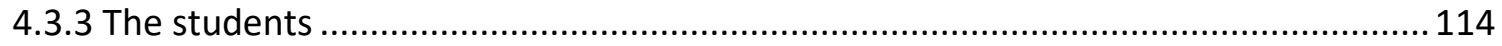

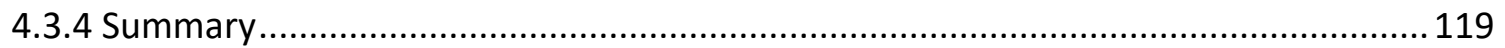

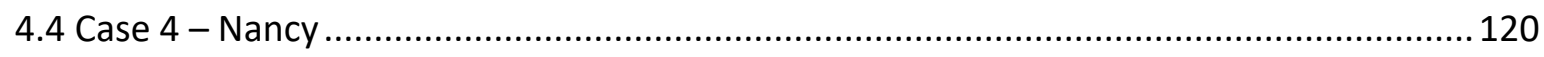

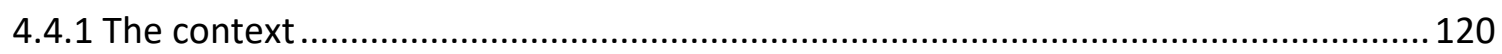




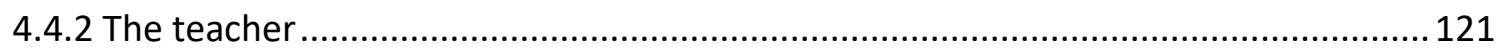

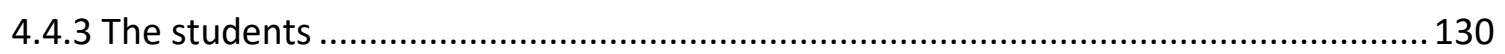

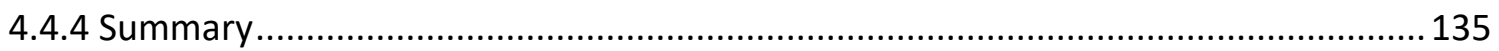

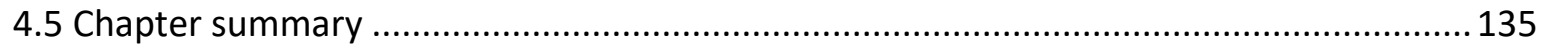

Chapter 5. Cross Case Analysis ...................................................................... 137

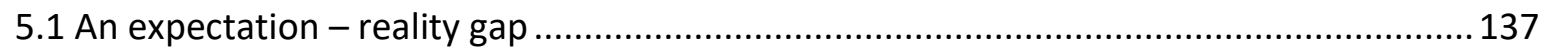

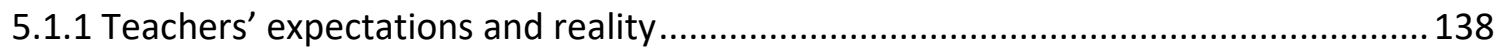

5.1 .2 Students' expectations and reality .......................................................... 141

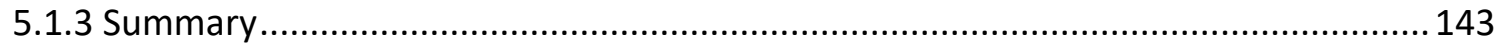

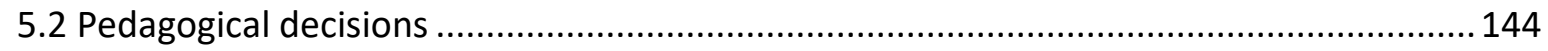

5.2.1 Making new content accessible to learners ................................................. 144

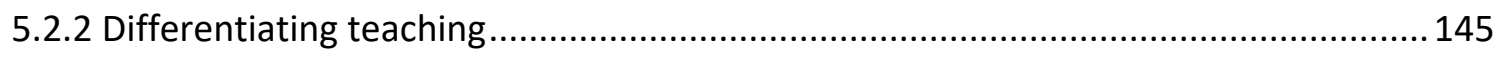

5.2.3 Facilitating students' analytical thinking and problem-based learning................... 148

5.2.4 Engaging students to collaborative and interactive learning ............................... 150

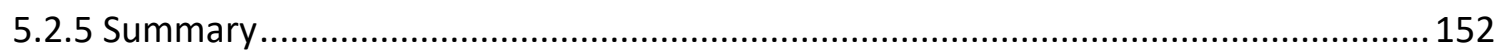

5.3 Integration of content, language and digital technologies influencing pedagogy ........... 155

5.3.1 Integrating English as a language of instruction into teaching subject matter ..........155

5.3.2 Integrating digital technologies into teaching subject matter ............................ 158

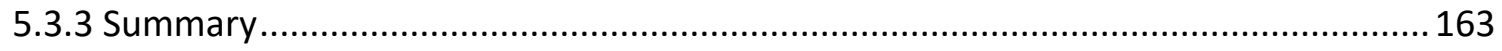

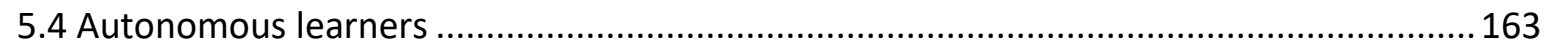

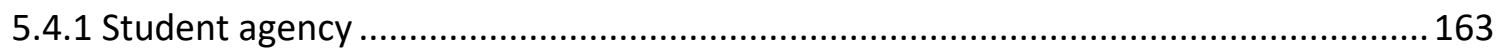

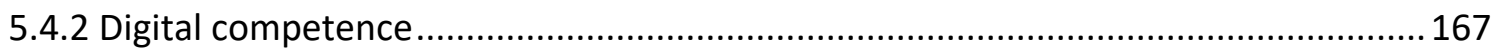

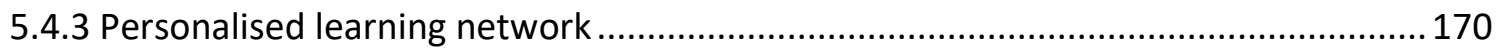

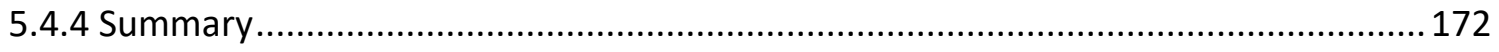

5.5 Summary of key findings in response to research questions ................................ 173

5.5.1 How do teachers and students use digital technologies in an EMI environment? ....173

5.5.2 How do teachers perceive their students' learning to have developed through digital technologies in an EMI environment?

5.5.3 How do students perceive their learning to have developed through digital technologies in the EMI environment? .......................................................... 174

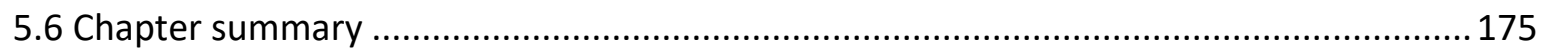

Chapter 6. Discussion.............................................................................. 177

6.1 The implementation of EMI programmes in the Vietnamese context........................177 


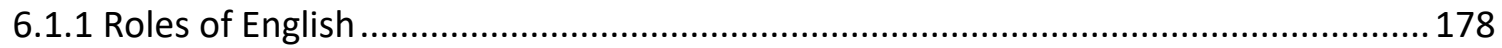

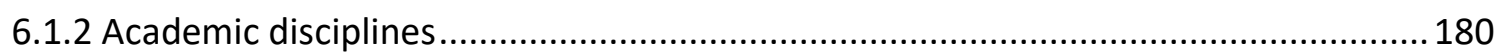

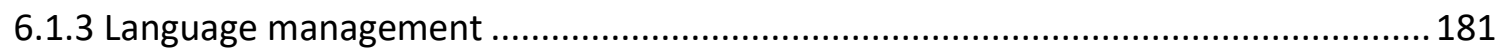

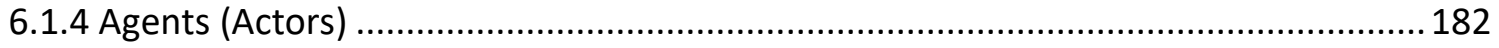

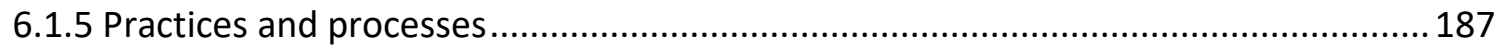

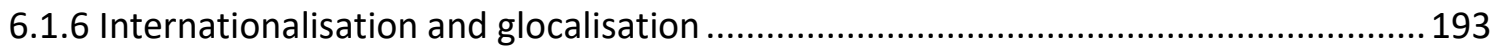

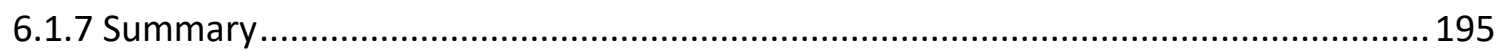

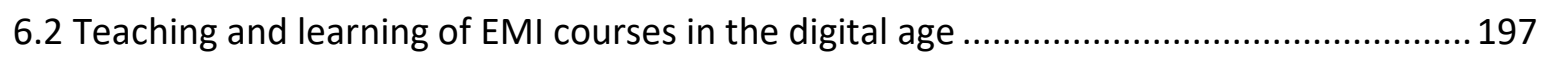

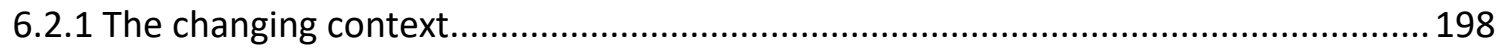

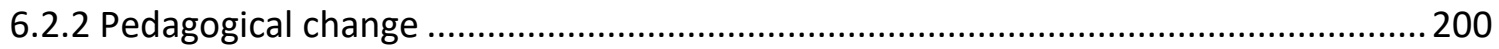

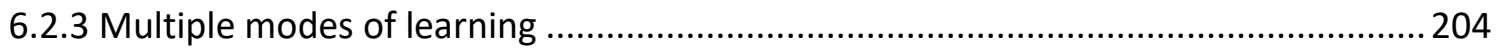

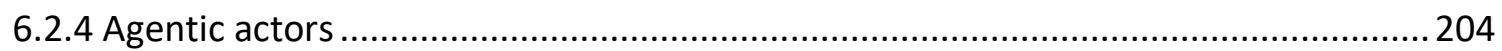

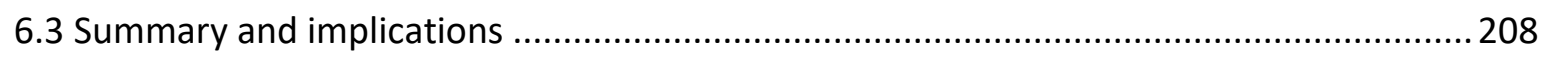

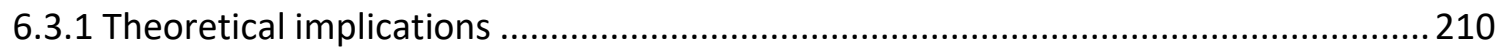

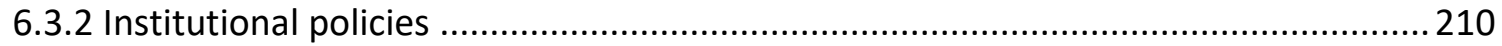

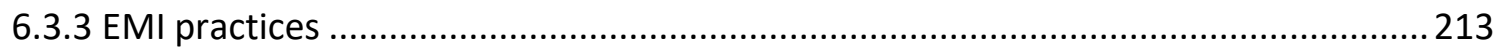

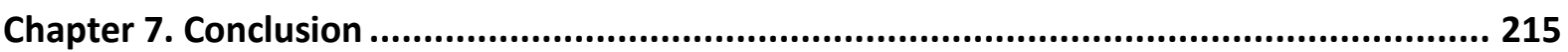

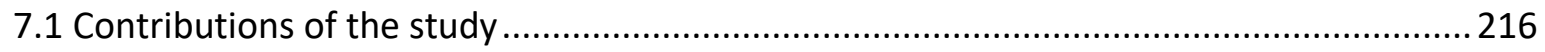

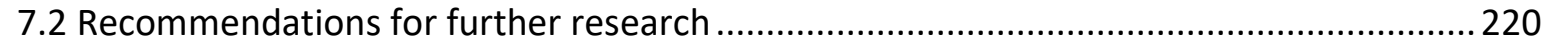

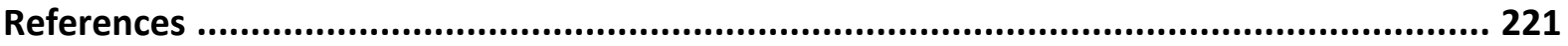

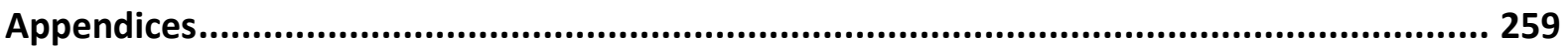




\section{List of tables}

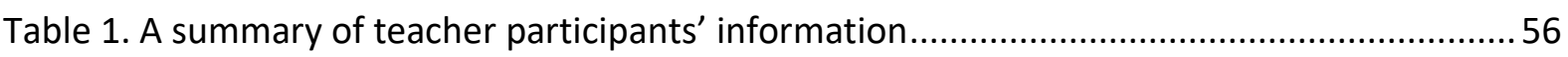

Table 2. A summary of students participating in focus group discussions ...................................57

Table 3. Dates of contacting participants and collecting data................................................... 58

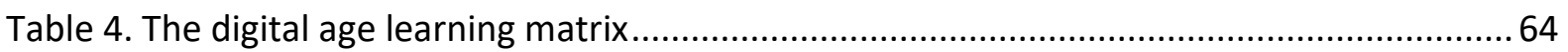

Table 5. The framework of principles in content-based language teaching and learning ............65

Table 6. Classroom observation (Vincent): Summary of teaching steps ...................................... 75

Table 7. Vincent's teaching activities compared to content-based English teaching principles ... 80

Table 8. Vincent's use of digital technologies to support her teaching ...................................... 81

Table 9. A summary of students' profiles regarding their confidence from the most (1) to the

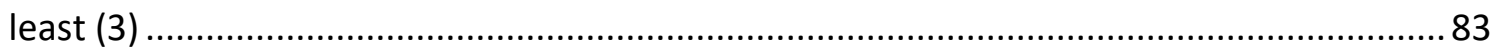

Table 10. Students' use of digital technologies in the digital age learning matrix ........................ 89

Table 11. Classroom observation (Nathan): Summary of teaching steps ...................................... 94

Table 12. Nathan's teaching activities compared to content-based English teaching principles 98

Table 13. Nathan's use of digital technologies to support his teaching .......................................99

Table 14. A summary of students' profiles regarding their confidence from the most (1) to the

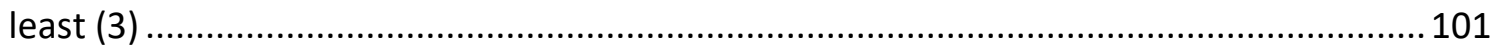

Table 15. Students' use of digital technologies in the digital age learning matrix ......................104

Table 16. Classroom observation (Taylor): Summary of teaching steps ...................................109

Table 17. Taylor's teaching activities compared to content-based English teaching principles. 112

Table 18. Taylor's use of digital technologies to support her teaching.

Table 19. A summary of students' profiles regarding their confidence from the most (1) to the

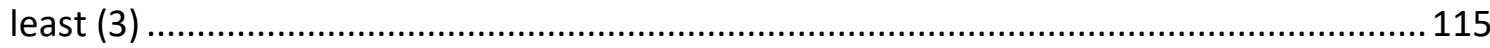

Table 20. Students' use of digital technologies in the digital age learning matrix .....................118

Table 21.. Classroom observation (Nancy): Summary of teaching steps ..................................123

Table 22. Nancy's teaching activities compared to content-based English teaching principles. 127

Table 23. Nancy's use of digital technologies to support her teaching.....

Table 24. A summary of students' profiles regarding their confidence from the most (1) to the

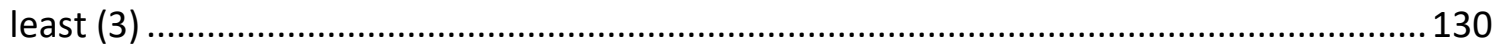

Table 25. Students' use of digital technologies in the digital age learning matrix ...................... 133

Table 26. The summary of the teachers' pedagogical decisions in an EMI economics course ... 153

Table 27. The teachers' use of English and Vietnamese in class .............................................158

Table 28. The teachers' use of digital technologies in their teaching .......................................162 


\section{List of figures}

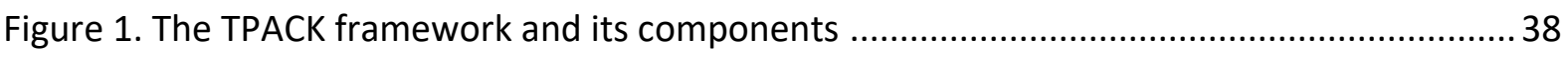

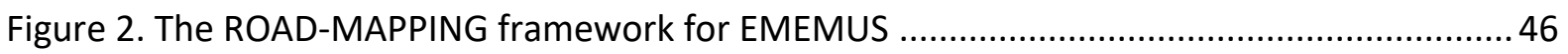

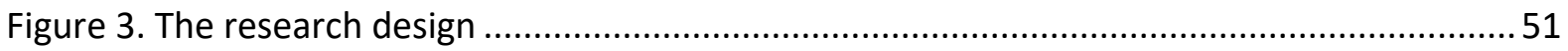

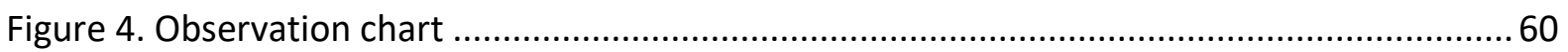

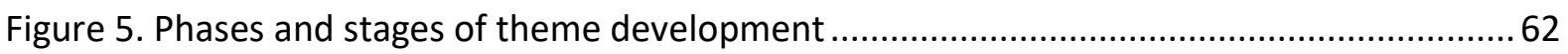

Figure 6. An example of the construction of potential themes................................................. 66

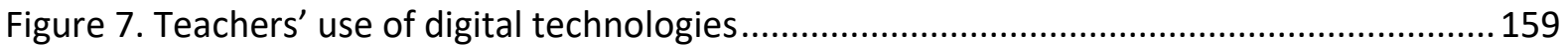

Figure 8. Students' digital competence demonstrated in their learning ...................................168

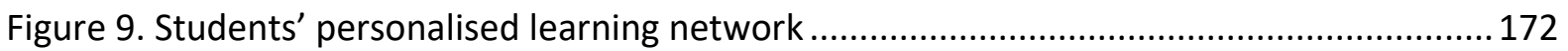

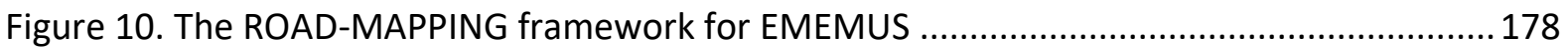

Figure 11. EMI in Vietnamese contexts through the ROAD-MAPPING framework.....................195

Figure 12. The changing context affecting pedagogical practices in the digital age ...................198

Figure 13. Digital technologies affecting EMI practices.........................................................213 


\section{Abbreviations}

CEFR

CLIL

EDO

EFL

EME

EMEMUS

EMI

ESP

ESS

GEPT

HE

ICLHE

ICT

IELTS

L1

L2

LMS

$\mathrm{MOI}$

NFL2020

PCK

TAM

TCK

TOEIC

TPACK

TPK

UTAUT

VLE
: Common European Framework of Reference

: Content and Language Integrated Learning

: English Discoveries Online

: English as a Foreign Language

: English-Medium Education

: English-Medium Education in Multilingual University Settings

: English-Medium Instruction

: English for Specific Purposes

: Education Support System

: General English Proficiency Test

: Higher Education

: Integrating Content and Language in Higher Education

: Information, Communication, and Technology

: The International English Language Testing System

: first language

: second language

: Learning Management System

: Medium of Instruction

: National Foreign Language 2020

: Pedagogical Content Knowledge

: Technology Acceptance Model

: Technological Content Knowledge

: The Test of English for International Communication

: Technological, Pedagogical, and Content Knowledge

: Technological Pedagogical Knowledge

: The Unified Theory Acceptance and Use of Technology

: Virtual Learning Environment 


\section{Chapter 1. Introduction}

Globalisation has influenced many sectors of society including higher education (HE) in different ways. Globalisation has led to developments in the knowledge industry, a burgeoning use of digital technologies, an increased mobility of people, and an orientation towards a market economy (Zajda \& Rust, 2016). The globalisation of HE is reflected through the integration of technologies and neoliberal policies in academic and administrative activities. HE institutions in a globalised world tend to be digitised through educational technology, social media, or artificial intelligence, and marketised "through performance-based appraisals, increasing competition between and within institutions, and pressures to reduce costs through the commodification of research outputs" (Larsen, 2016, p. 397). This has resulted in changes in HE contexts which influence teachers' and students' academic practices.

Hand in hand with globalisation, the process of internationalisation has also gained much attention in the HE sector. Universities have created strategies to "develop and strengthen relations across national borders" (Larsen, 2016, p. 398). These strategies are associated with increased student intake, recruitment of international students, promotion of institutional profiles and ranking, enhanced international collaboration in research and curriculum development, and a new focus on foreign language instruction (Delgado-Márquez et al., 2013). Accordingly, developing competency in English as a global language is viewed as an essential strategy in the process of internationalisation.

More and more universities are implementing English-medium instruction (EMI) programmes, "an educational system where content is taught through English in contexts where English is not used as the primary, first, or official language" (Rose \& McKinley, 2018, p. 114). Given that many HE institutions are using "Englishisation" (Dafouz \& Smit, 2020) to internationalise their programmes, EMI has become intertwined with internationalisation (Kirkpatrick, 2011). This worldwide trend includes multilingual Asian countries (Kirkpatrick, 2011) such as Vietnam (Tran \& Nguyen, 2018).

My thesis explores EMI implementation in the digital age in a Vietnamese context. I conducted case study research at one Vietnamese university which implemented EMI programmes in their mainstream curriculum. I investigated how teachers and students experienced teaching and learning in EMI courses, and how and why they used digital technologies for academic purposes in the context of EMI. To do this, I collected data from four teachers and four groups of students in EMI courses through semi-structured interviews, classroom observations, and student focus-group discussions. The findings provide insight into EMI practices and the integration of digital technologies 
into teaching and learning activities in the context of a Vietnamese university in times of globalisation and heightened internationalisation.

\subsection{Research context}

Vietnam is the third largest nation in Southeast Asia sharing borders with China in the north, Laos in the northwest, and Cambodia in the southwest. It is a multilingual country with a population of almost 96 million people and 54 ethnic groups. Vietnam is a one-party socialist country officially espousing communism but with a market economy. The National Assembly is the highest state power of the Socialist Republic of Vietnam and it is the sole organisation for assuring the people's constitutional and legislative rights. Confucianism is the foundation of Vietnamese culture and this "national philosophy in politics, education and social life of the Chinese people" (MacKinnon \& Le, 2014 , p. 149) is long-established in Vietnamese society. Confucian values such as the appreciation of harmony and stability, the importance of power distance, and collectivism influence the beliefs and behaviours of many Vietnamese people. In recent decades, while Western cultures and the Communist Party of Vietnam have begun to influence Vietnamese culture, the core values of Confucianism is still dominant in Vietnamese society (Truong et al., 2017). The Vietnamese education system is an area reflecting Confucian values.

Political, cultural and economic values are strongly mirrored in the education sector. Politically, all educational institutions must comply with the national laws and regulations. For example, in $\mathrm{HE}$, the National Assembly issued the HE law (2002) that details principles for the operation of tertiary institutions. Every university has to set their policies in compliance with this. Culturally, Vietnamese education reflects values of Confucianism such as a desire for whole-life learning, an interest in repetitive learning, and limited interest in practical activities (Tran, 1995, as cited in MacKinnon \& Le, 2014). Despite the recent influence of Western individualism, Vietnamese culture maintains its traditional focus on humanity and harmony. This shapes the identity and worldviews of the Vietnamese people toward education, especially HE (MacKinnon \& Le, 2014). With respect to economic values, since the country's Doi Moi (Economy Renovation or Reform) in 1986, Vietnam has opened its economy to the world and established relationships with different countries. Participating in the World Trade Organisation and committing to the General Agreement on Trade in Services has seen Vietnam's rapid economic growth through developing international trade and a number of private, joint venture and foreign direct investment companies. This has not only facilitated the integration of Vietnam into the global market but has also raised demand for a 
workforce with multilingual competence. As a result, the government has set new objectives for educational institutions focussing on the process of internationalisation and the promotion of English language teaching and learning.

The HE system has undergone significant changes over the last decades to become a cornerstone of transformation in Vietnam. Since Doi Moi, HE institutions have adapted in response to globalisation and neoliberalism, marketisation, and technological development. Universities have gained more autonomy and moved away from the subsidised system (Pham \& Fry, 2004), to diversifying their income "by introducing tuition fees, commercialisation of research activities, and massification of HE” (Nguyen, 2009, p. 2). Recently, the process of massification in HE has been reflected in a vast increase in student enrolments "from roughly 133,000 in 1987 to more than two million in 2010" (Do \& Do, 2014, p. 51). However, neoliberalism and growing market competition have put pressure on university authorities, academic programmes, financial resources, and student recruitment as universities try to respond to these changes. This pressure was intensified by the $H E$ Renovation Project for the 2006-2020 period, where the Ministry of Education and Training stated that HE needs "better fulfil its mission of training highly qualified human resources to meet the country's socio-economic development requirements and people's learning needs" (Nguyen \& Nguyen, 2008, p. 20). This called for the reform of the HE system in Vietnam.

In the Strategy of Education Development for Vietnam 2011-2020 issued by the Prime Minister (2012), internationalisation is considered as one of eight specified initiatives for enhancing the development of the nation's HE sector. The process of internationalising HE institutions is expected to enhance "the capacity and standard of the HE system, assist universities to keep pace with international and regional developments", and facilitate Vietnam in the augmentation of human resources and integration in the globalised world (Tran \& Nguyen, 2018, p. 94). In order to follow this process, universities have strategised expanding and enhancing international collaboration in delivering and developing academic programmes, as well as supporting student and staff mobility (Nguyen \& Tran, 2018). Accordingly, focus has been paid to the internationalisation of curriculum via transnational curriculum. To do this, Vietnamese HE institutions commonly import curricula from foreign English speaking partner universities, which has led to the increasing use of EMI to achieve internationalisation goals (Tran \& Nguyen, 2018).

The promotion of EMI is crucial to the internationalisation of HE in Vietnam. The implementation of EMI offers Vietnamese universities opportunities to enhance the quality of their 
teaching and learning, promote an institutional profile as multilingual and international-focused, and attract more domestic and international students. This was officially initiated in the National Foreign Language 2020 (NFL2020) project of promoting English capability promulgated by the Vietnamese government. In this project, five core objectives were mandated for the education system including: (1) establishing a proficiency framework compatible with the Common European Framework of Reference (CEFR); (2) implementing compulsory English language education from Grade 3; (3) instituting English as a Medium of Instruction (MOI) for maths and science in upper secondary schools; (4) improving English teachers' English language proficiency and understanding of language pedagogy and language acquisition; and (5) delivering programs in English at selected universities (Vietnamese Government, 2008). To meet the last objective, several Vietnamese universities have mandated EMI programmes in their curriculum (Nguyen et al., 2017).

Different types of EMI programmes have been implemented in Vietnamese HE. English as a medium of instruction was first introduced and implemented in the form of Content and Language Integrated Learning (CLIL) for primary and secondary students in 1998, and EMI was first adopted in programmes taught jointly by domestic and foreign HE institutions in the early 1990s (Nguyen et al., 2017). This type of foreign EMI-based programme is called Chương trình Liên kết (Joint Programmes) and aims to attract Vietnamese students wanting to experience foreign style tertiary programmes and gain overseas qualifications provided at domestic HE institutions (Tran \& Nguyen, 2018, p. 98). Another type of EMI programme called Chương trình Tiên tiến (Advanced Programmes) was developed through cooperation between domestic and foreign universities in which the degree was awarded by the domestic university. The third type is the domestic EMI-based programme developed by domestic universities in which they coordinate foreign programmes and apply curriculum, materials, and assessment schemes developed by foreign universities. These programmes are called Chương trình Đào tạo Chất lượng cao (High-Quality Programmes) (Tran \& Nguyen, 2018). These three types of EMI programmes are now implemented in HE institutions in Vietnam.

There are challenges in adopting EMI in Vietnamese universities. One issue is associated with the English language proficiency of both teachers and students taking EMI courses (Vu \& Burns, 2014). Limited English competence has the potential to inhibit teachers' ability to teach in English or students' ability to understand content taught in English. Moreover, there is little evidence in literature that EMI has a positive impact on students' language competence and academic performance (Tran \& Nguyen, 2018). In addition, the lack of EMI materials and professional 
development for EMI teachers has also challenged the promotion of EMI programmes (Dang \& Moskovsky, 2019; Vu \& Burns, 2014). It would appear that even though EMI is in the process of implemented in Vietnamese HE institutions with the aim of improving both content and English, English tends to be overlooked. This highlights the need for a comprehensive investigation of how EMI is actually implemented in Vietnamese universities.

The integration of digital technologies in Vietnamese education has also been promoted by educators and policymakers. Several factors have been found to affect how teachers integrate technology into their classes such as teachers' intensity of use, their related skills, their confidence in using ICT, their conceptions of learning, and their access to ICT (Peeraer \& Van Petegem, 2011). Over the last two decades, specific guidelines and policies associated with technology usage in education have been put in place to address these issues (Peeraer \& Van Petegem, 2015). The Ministry of Education and Training Directive No. 9772/2008/CT-BGDDT (2008) required institutions to strengthen Information and Communication Technology (ICT) use in teaching and training. The ICT Development Index shows that access to ICT in Vietnam has significantly improved since 2000. This aligns with the Southeast Asian Ministers of Education Organization (SEMEO) report (2010) that policy guidelines and ICT infrastructure and resources in Vietnam should support schools to transform teaching and learning practices with technology. Those crucial factors have the potential to enable the integration of technology in education in Vietnam (Peeraer \& Van Petegem, 2015).

However, it is unclear how teachers use technologies in academic practice in Vietnam. University lecturers are expected to develop skills and knowledge to teach with digital technologies (Harman \& Nguyen, 2010). Yet, it has been shown that teachers do not use ICT in their courses even when they are aware of the potential benefits (Ly \& Habibah, 2013). When they do use technology, Vietnamese teachers "mostly use ICT in ways that mainly replace traditional teaching practice" (Peeraer \& Van Petegem, 2015, p. 47). In the $21^{\text {st }}$ century, when digital technologies have the capacity to be integral to students' learning experiences (Selwyn, 2016), teachers and students are expected to enhance teaching and learning by regular, innovative use of digital technologies (Peeraer \& Van Petegem, 2015). This raises the question of how university teachers and students actually use digital technologies to meet this $21^{\text {st }}$ century need. To answer this question, I have investigated how teachers and students at a Vietnamese university used digital technologies for teaching and learning in EMI courses. 


\subsection{Rationale for the research}

I had nearly ten-years' experience of working as an English as a Foreign Language (EFL) teacher in Vietnam before commencing my PhD journey. In my teaching, I often used a range of digital technologies for various purposes. Specially, I recognised that integrating technologies into teaching activities helped me stimulate students' interest, enhance their engagement, and motivate their participation in learning. This prompted me to explore more about the use of digital technologies in English teaching and learning. As such, I started my PhD with a research interest in the field of educational technologies.

As an English teacher at a Vietnamese business university, I took responsibility for teaching business English to students majoring in different areas of economics. This role provided me with experience in teaching English for specific purposes (ESP). It was a challenging job to balance teaching language and subject content. In those ESP courses, English teachers had to teach students English terms for business concepts and reading skills so that students could read texts in their specialism. Yet, students sometimes asked me for a more comprehensive explanation of economics concepts. I personally found it hard to take the role of subject teacher at that time. This explains why I am aware of how challenging it is for subject teachers and students to teach and learn in a CLIL/EMI setting. Drawing on my personal experience, I wondered how teachers and students were adapting to the emerging context of CLIL/EMI and whether digital technologies could be used to enhance their teaching and learning practices in that setting.

\subsection{Research question}

In this study, I investigate the ways in which digital technologies were used for learning and teaching in EMI classes. My major research question is:

\section{How are digital technologies used in an EMI context in Vietnamese higher education?}

The following sub-questions support this main research question:

1. How do teachers and students use digital technologies in an EMI environment?

2. How do teachers perceive their students' learning to have developed through digital technologies in an EMI environment?

3. How do students perceive their learning to have developed through digital technologies in the EMI environment? 


\subsection{Structure of the thesis}

My thesis consists of seven chapters. This chapter introduces the topic, describes the context of Vietnamese $\mathrm{HE}$, and explains the rationale for the study.

Chapter 2 reviews key literature relevant to the study. It focusses on studies of how content and language are integrated in the HE sector and teaching and learning in the digital age. In this chapter, I also introduce the conceptual framework underpinning the interpretation of the study findings. Following this, Chapter 3 provides a detailed description of the qualitative method used to collect and analyse data gathered through interviews, classroom observations, and focus group discussions.

Chapters 4 and 5 report and analyse the findings of the study. Chapter 4 describes the four individual cases, and Chapter 5 synthesises significant themes from cross-case analysis. Chapter 6 discusses the implementation of EMI in Vietnamese HE based on the ROAD-MAPPING framework and addresses the teaching and learning of EMI courses in the digital age. This chapter also provides some implications associated with institutional policies, EMI practices, and theory. Finally, Chapter 7 concludes the study with a brief summary of findings, discussion of limitations, and proposes issues for further research. 


\section{Chapter 2. Literature review}

This chapter reviews literature related to the implementation of Content and Language Integrated Learning (CLIL) / English-medium instruction (EMI) programmes and the use of digital technologies in HE contexts. The review starts with a discussion of studies exploring the integration of content and language in $\mathrm{HE}$ before detailing studies on the integration of digital technologies into university teaching and learning. The chapter finishes with a description of the conceptual framework underpinning the adoption of EMI in HE.

\subsection{Integrating content and language in higher education}

The integration of content and language has become a phenomenon in HE over the last decades. In response to the influence of internationalisation and globalisation, many universities

globally promote the use of an additional language (very often English) as a medium of instruction in their academic programmes (Dafouz \& Smit, 2016). As discussed in Chapter 1, these programmes are designed for different purposes such as attracting international students, preparing domestic students for the global labour market, and raising the profile of the institution (Doiz et al., 2011). This section synthesises findings from studies addressing aspects of CLIL and EMI, starting with the international context, and then moving to the Vietnamese HE setting.

\subsubsection{CLIL and EMI in higher education}

CLIL and EMI have been the focus of recent research conducted across all phases of education (Dearden, 2014). In the university context, findings are unsurprisingly divergent and varied "due to the heterogeneous contexts under study" (Aguilar, 2017, p. 73). To shed light on this complexity, I first discuss important terminology, before highlighting the motives and stakeholders' attitudes towards the implementation of CLIL and EMI in $\mathrm{HE}$, and finally exploring the literature related to the language learning, the content learning, and the teaching and learning strategies used in CLIL and EMI settings.

\subsubsection{Terminology}

In research, different labels have been used to address the phenomenon of integrating language into the teaching and learning of content knowledge. For example, researchers have used terms such as Content and Language Integrated Learning (CLIL), English-medium teaching, Englishmedium instruction (EMI), and Integrating Content and Language in Higher Education (ICLHE) (Smit \& Dafouz, 2012). While some authors have used these terms interchangeably, others distinguish 
them according to specific conceptualisations of each term. Coyle et al. (2010) suggest the option of a dual focus educational approach to show that a fusion of both content and language is required. According to these researchers, any teaching approaches that lack "such fused pedagogical teaching aims" should not be considered as content and language integrated learning programmes (Smit \& Dafouz, 2012, p. 7). Another distinction mentioned in Aguilar (2017) is that CLIL is popularly used for primary and secondary education research while EMI, ICLHE and CLIL are common in HE . However, no matter what terms are used, there remains the need to thoroughly understand the actual implementation of academic programmes in which disciplinary content is taught and learnt through an additional language.

These terms, especially CLIL and EMI, albeit with common aims have conflicting definitions. CLIL, developed in 1994, is defined as "a dual-focused educational approach in which an additional language is used for the learning and teaching of both content and language" (Coyle et al., 2010, p. 1). In this approach, content refers to the subject matter related to various learning areas, and a foreign language is used as the medium of instruction. CLIL requires teachers to equally emphasise both language education and subject education and this is flexibly implemented in a wide range of contexts and education systems. EMI shares similar aims but is defined more broadly as "the use of the English language to teach academic subjects (other than English itself) in countries or jurisdictions where the first language of the majority of the population is not English" (Macaro et al., 2018, p. 37). This indicates that English is normally the "medium" for delivering the content however, there may be "no direct reference to the aim of improving students' English" (Dearden \& Macaro, 2016, p.456). In practice, regardless of how the policy is defined, EMI courses in many higher education institutions tend to emphasise academic content at the expense of explicit language learning aims (Brown \& Bradford, 2016). This interpretation of EMI conflicts with Taguchi's (2014) definition as "curricula using English as a medium of instruction for basic and advanced courses to improve students' academic English proficiency" (p.89). This definition suggests that EMI is intended to support English language learning. This understanding of EMI is aligned with the policy of promoting EMI in tertiary levels with the aim of equipping students with academic skills to compete in the global market where English is the means of communication (Bradford, 2015). In this sense, there remains a question whether and how explicit or implicit aim of language teaching and learning should be incorporated in the practice of implementing EMI in higher education. These conflicting definitions reveal varying forms of EMI practices in reality. 
Both definitions of CLIL and EMI are appropriate for my study because the university offers academic programmes in economics taught and learnt through English as a medium of instruction aiming to promote both content and English. However, the term EMI predominated in their policies, so I mainly refer to EMI in my study.

\subsubsection{The implementation of CLIL/EMI in higher education.}

In this part, I will review literature on the implementation of CLIL/EMI in HE by discussing how more and more universities to adopt CLIL/EMI in their academic programmes and addressing findings of previous research focussing on different issues associated with CLIL/EMI programmes.

\section{Motives behind the implementation}

Researchers have distinguished a number of motives for the implementation of CLIL and EMI programmes in HE. As mentioned earlier, Doiz et al. (2011) found three major reasons for CLIL/EMI adoption including attracting international students, preparing domestic students for the global labour market, and raising the profile of the institution. Supporting this, Wilkinson (2012) reported five motives behind the decisions by institutions to establish EMI programmes: practical, survival, financial, idealist, and educational. Specifically, he illustrated his point with an example of how different phases of EMI programmes at Maasstricht University were implemented for different purposes, such as: recruiting international and exchange students (practical), profiling the institution as international (survival), dealing with the cost of bilingual options (financial), promoting internationalisation at home (idealist), and introducing new academic programmes (educational).

These institutional motives vary in different contexts. For instance, the policy makers in Turkish universities considered EMI programmes as tools to promote their universities' image as 'elite' and guarantee the quality of their student intake (Başıbek et al., 2014). The use of English as the instructional language was in this case also a tool to achieve the goal of internationalisation set by state universities (Başıbek et al., 2014). This is similar to what Cho (2012) found in his study of the adoption of EMI in HE in Korea. In this context EMI was promoted to have a positive impact on mediainitiated university rankings of internationalisation as part of a worldwide globalisation process. In other words, the university implemented EMI to "secure a high rank in university rankings or to promote the brand name of the school" (Cho, 2012, p. 158). These policies mainly arise from topdown university-level strategies, which do not appear to include consultation with other key stakeholders such as teachers and students (Dearden \& Macaro, 2016). In Cho's (2012) study of faculty staff and students, $52.9 \%$ of teachers responded that "they lectured in English to meet the 
EMI policy by the school" (p. 145). In Taiwan, Yeh (2014) also researched the experiences and attitudes of 476 students towards EMI courses and found that $22 \%$ of them reported having few or no choices, as they were required to attend EMI courses.

Teachers and students report different motivations for participating in CLIL/EMI programmes. Primarily, they recognise the significant role of English as a lingual franca in today's world. Findings from Belhiah and Elhami's (2015) research showed that both teachers and students believed that learning in English was necessary as English is a global language that prepares students for competition in the job market. This is similar to Al-Masheikhi et al.'s (2014) findings that studying in English would help students to get a good job in the future (Al-Masheikhi et al., 2014). What is more, the students insisted on the necessity of learning their subjects in English as they believed "English is the dominant language of science and technology" (p. 106). This result was consistent with Karmani's (2010) findings from his study in which the students thought that English was the most appropriate language of instruction for learning the subjects of business studies and information technology.

Access to English-medium materials is a further motivation for teachers and students' involvement in CLIL/EMI and is believed to make a difference in the successful implementation of EMI. For example, Turkish lecturers supported the implementation of EMI programmes because materials in English were easily available and accessible and supported students in their field of study (Başıbek et al., 2014). Bangladeshi teachers had a similar experience, noting the availability of Englishmedium textbooks and other teaching and learning resources as supporting learning in EMI classes (Hamid et al., 2013, p. 153). In Korea too, required courses and accompanying course books were only available in English (Kang \& Park, 2005). As such, the availability of English used in teaching and learning materials appears to play a significant role in promoting the adoption of CLIL/EMI programmes.

The lecturer's reputation as an expert in the field may influence student enrolment (Yeh, 2014). In other words, many students tend to enrol in courses taught by lecturers who are known for their content knowledge of the discipline. In Yeh's study, a considerable percentage of student participants (47\%) selected courses based on the instructor's expertise in the subject matter, and only $19 \%$ paid attention to the instructor's teaching methods or teaching style. This means that the students did not consider how the lecturers would teach the subject when the language of instruction was changed to English. Additionally, many students decided to take part in CLIL/EMI courses so that in the future they would be able to more easily pursue further study, especially at foreign university 
(Al-Masheikhi et al., 2014). Finally, both lecturers and students valued English as a "commanding language" which could make the students appear sophisticated, smart, or highly-educated (Hamid et al., 2013, p. 155). In this sense, participating in CLIL/EMI would give students a more highly appreciated image compared those students learning in their first language. Similarly, some lecturers defined themselves as international teachers when they are able to teach their subjects in both English and their first language, appealing to these same discourses of linguistic prestige.

In summary, the literature suggests that stakeholders, including policy makers, teachers, and students, had different motivations for participating in CLIL/EMI programmes. They were all aware of the benefits - content knowledge and English competence - that were likely to accrue from the implementation of CLIL/EMI. University authorities expected that CLIL would promote their institutions' brands, enhance their rankings to attract more good students, and achieve the goal of internationalisation. Meanwhile, many teachers and students adopted CLIL/EMI courses to meet the policy requirements though they expressed different motives for their decisions including the role of English as a lingua franca and a dominant language in fields like science, engineering, technology or business, professional opportunities in the global job market, the availability of teaching and learning materials in English, the reputation of the lecturers in their field, the opportunity for overseas study, and the added-value to their own image.

\section{Attitudes of teachers and students towards CLIL/EMI implementation}

Many studies have explored the attitudes of teachers and students towards the implementation of CLIL/EMI programmes. In general, both teachers and students participating in CLIL/EMI expressed their support of the programmes and believed that they could receive various benefits. For example, the teachers and students participating in Ghorbani and Alavi's (2014) research supported the application of EMI at Iranian universities as they appreciated the potential advantages including the opportunity to introduce Iranian culture and religion to the world, to increase the quantity of international academic publications, and the prospect of improving the students' levels of English proficiency. Similarly, Islam (2013) found that the teachers and students in a Bangladeshi university generally favoured the opportunity for future careers in the global market that the adoption of EMI could facilitate. However, teachers and students held some concerns about the effectiveness of CLIL/EMI. Typically, they felt concerned about the students' content comprehension and improvement in English (Islam, 2013) as the students' limited English proficiency was an obstacle that had the potential to hinder students' success in education (Borg, 2016). In Kim and Shin's (2014) 
study on 48 lecturers and 116 students in Korea, the participants "evaluated the English communication ability of approximately $30 \%$ of students to be ill-prepared for EMI classes" (p. 48). As such, teachers and students did not completely believe that EMI classes were as effective as those taught in their first language (Cho, 2012).

Regarding CLIL/EMI teachers' perspectives, some research highlights teachers' feelings of doubt related to their preparation for CLIL/EMI courses (Werther et al., 2014). Although teachers reported some positive consequences of the CLIL/EMI policy in attracting foreign students, promoting the use of English, increasing students' job opportunities, and facilitating the teachers'/students' chances to participate in exchange programmes (Başıbek et al., 2014; Doiz et al., 2011), they did not feel confident to participate in teaching through another language. For example, Borg (2016) surveyed 416 lecturers teaching in English at 13 universities and found that the participants were most concerned about their own English proficiency even though they felt that they were able to teach their subjects in English (Borg, 2016). Likewise, Dearden and Macaro (2016) revealed that many teachers at universities in Austria, Italy, and Poland were not confident and felt pressure to teach effectively in a second language. Even young confident teachers who were proficient in English felt "unsure whether they had a sufficient level of English to teach through EMI" (Dearden \& Macaro, 2016, p. 471). Moreover, teachers are reluctant to the possible impact of using a foreign language on their teaching. For instance, Thøgersen and Airey (2011) revealed that the lecturers "take $22 \%$ longer to present the same content in L2 compared to L1, and that the lecturers speak $23 \%$ more slowly in L2 than in L1" (p. 209). The register was also affected. These lecturers used more formal language structures in their English language lectures than those in Danish (Thøgersen \& Airey, 2011). Albeit with positive attitudes, teachers still had concerns about their ability to teach their subjects through an additional language.

An array of research has focussed on teachers' attitudes towards the policy of implementing CLIL/EMI into academic curriculum. For instance, in Malaysia, Ali (2013) examined EMI policy in HE by interviewing one university executive and 11 lecturers. The findings revealed that the contentarea teachers did not feel ready for teaching their subjects through English. The lack of thorough guidelines in EMI implementation created confusion and difficulties for them in their teaching (Ali, 2013). In Korea, Choi (2013) interviewed nine professors in a regional private university and found that many participants had negative experience in EMI courses as they were required to spend a great deal of time developing new teaching approaches and techniques, maintaining a strong focus on 
students' English improvement, and dealing with students' limited English proficiency (Choi, 2013). Likewise, in Airey's (2011) study, 18 lecturers from two Swedish universities reported similar challenges when teaching content courses in English, including insufficient training, the demands of more preparation, or changes in teaching performance. As a result, a number of teachers oppose the policy of adopting CLIL/EMI into academic programmes (Cho, 2012).

Research has also explored the implementation of CLIL/EMI from the students' perspective. Students in many studies reported feeling highly motivated to attend CLIL/EMI classes. They believed in the potential of these classes to improve their English skills, to make international friends, to provide future opportunities to pursue their study abroad, and to facilitate their employability. In Yeh's (2014) study, the results showed that the students felt satisfied with their EMI experiences and agreed that EMI courses had a positive impact on their English skills, English learning motivation, and learning of content knowledge. The participants generally expressed favourable opinions about EMI courses and revealed their willingness to take more EMI courses (Yeh, 2014). Huang's (2015) study of 157 Taiwanese students had similar findings. Most participants felt motivated by and agreed on the merits of EMI courses in strengthening their English ability and professional knowledge (Huang, 2015). These findings are also consistent with what was found in Macaro and Akincioglu's (2018) survey of 989 students in Turkey. Despite variations in students' year groups, gender, and university types, the participants shared positive attitudes towards the benefits they believed EMI would bring, especially regarding improvement in English proficiency. They also felt satisfied with other aspects of EMI programmes such as the resource availability, and the quality of teaching (Macaro \& Akincioglu, 2018).

Nonetheless, the students experiencing CLIL/EMI recognised an array of challenges in practice. In Turkey, Bozdoğan and Karlıdağ (2013) explored the reflections of 15 voluntary and found that even though the students considered that CLIL fostered their English proficiency, it was challenging to understand the subject content in English and difficult dealing with subject terminology in English. More negatively, the students felt that the CLIL course curricula were simpler than those in their first language, and their productive skills in English were even regressing (Bozdoğan \& Karlıdağ, 2013). Similar findings were mentioned in Evans \& Morrison's (2011) research at Hongkong Polytechnic University which revealed four major problems including "understanding technical vocabulary, comprehending lectures, achieving an appropriate academic style and meeting institutional and disciplinary requirements" (Evans \& Morrison, 2011, p. 198). 
Researchers have found that students' preference for CLIL/EMI programmes was associated with factors such as type of programmes, students' year, gender, and type of university. For example, in Thailand, a large-scale study was conducted to investigate students' motivation and their choice of EMI programmes with the participation of 2252 undergraduate students enrolled in nine different academic programs (Hengsadeekul et al., 2014). The results showed that academic programmes influenced students' motivation and preference for EMI programmes. In particular, the students who majored in international program business, English, and nursing would prefer English-medium graduate programmes more than those in IT, business, and vocational studies (Hengsadeekul et al., 2014). This indicates that CLIL/EMI has discipline-specific appeal. In Macaro \& Akincioglu's (2018) study, three factors: students' year group, gender, and university type were taken into consideration. The findings revealed that although the students in all three years felt motivated to enrol in EMI programmes, female students and those attending private universities were more certain about the benefits of the EMI courses. What is more, the private university students expressed more positive attitudes and higher levels of satisfaction towards their experiences in EMI (Macaro \& Akincioglu, 2018). This might be due to the difference in students' English proficiency as well as students' preference for English. As such, all of these factors need thorough consideration in the process of implementing CLIL/EMI into academic programmes.

In summary, teachers and students generally have positive attitudes towards the implementation of CLIL/EMI programmes. They perceive various advantages that CLIL/EMI is likely to bring them in terms of opportunities for future career, English development, and academic publications. However, the literature also points to a number of challenges. For teachers and students in CLIL/EMI courses, these include English proficiency, their preparation and readiness for the programmes, and the policy of adopting CLIL/EMI itself. Factors affecting students' preference for CLIL/EMI programmes include the type of programme, year of study, gender, and type of university.

\section{Language learning in CLIL/EMI settings}

CLIL/EMI, as a dual focus approach, is considered to facilitate language proficiency (Tai, 2015). CLIL/EMI students theoretically have more exposure to the foreign language than their non-CLIL/EMI peers as they "nearly always continue with their regular foreign language program alongside CLIL/EMI content lessons" (Dalton-Puffer, 2011, p. 186). This suggests that learners in CLIL/EMI contexts are likely to have improved language outcomes. Some researchers, however, have identified a lack of explicit language instruction in CLIL/EMI classes (Dalton-Puffer, 2008; Lasagabaster, 2011), which cast 
doubts on CLIL/EMI's effectiveness (Bruton, 2011). Several studies have investigated different aspects of language learning in CLIL/EMI programmes such as the acquisition of lexical phrases (Catalan \& Fontecha, 2015), receptive vocabulary (Admiraal et al., 2006), writing (Huges \& Madrid, 2015; Jexenflicker \& Dalton-Puffer, 2010), morphosyntax in speaking (Lázaro, 2012), or listening and grammar skills (Aguilar \& Muñoz, 2014). Despite reports of positive results, there has been scant empirical research measuring the impacts of CLIL/EMI on English learning or English proficiency in $\mathrm{HE}$ (Macaro et al., 2018).

A few studies, albeit a limited number, have used specific tools to measure the effectiveness of CLIL/EMI in students' language learning. While much research reports the positive impacts of CLIL/EMI on language improvement based on self-perception reports, there is a need for research findings to be confirmed by the use of different tools. For instance, Pessoa et al. (2014) conducted a 4-year longitudinal study at an English-medium university in Qatar to investigate students' literacy skills on transition to college. The researchers analysed 86 students' academic writing scripts using the DocuScope software, a dictionary-based programme for text analysis. Also targeting students in transition to university education, Lin and Morrison (2010) explored academic vocabulary size during a period when most secondary schools in Hongkong had changed from English-medium instruction to Chinese-medium instruction. They used the Vocabulary Levels tests (Schmitt et al., 2001) to measure the receptive and productive vocabulary of 762 students, then applied an online computer programme called Vocabprofile (Cobb, 2009) to assess the lexical richness of 413 essays written by volunteered participants, and finally asked an experienced language instructor to access the lexical appropriacy of those essays. Sharing the same focus, Tai (2015) examined 57 written assignments submitted by 19 students in an 18-week CLIL class. He used T-units (Hunt, 1966) to analyse the syntactic complexity, syntactic accuracy, and fluency of the participants' writing throughout the short course. In another Taiwanese university context, Yang (2015) measured the English performance of learners in CLIL programmes using pre-tests and post-tests which were simulated General English Proficiency Tests (GEPTs). Similarly, Rogier (2012) rated the English language skills development of students in UAE universities by using a test/retest method and compared students' IELTS scores after four years of studying in EMI programmes. In Spain, Aguilar and Munoz (2014) undertook research with 205 students in a CLIL course at an engineering school to examine the impact of CLIL on learners' listening and grammar skills using the Oxford Placement Tests as pre-test and post-test. By using different kinds of tests or measuring applications, these studies have been expected to produce 
persuasive results related to the effectiveness of CLIL/EMI programmes in terms of student language learning.

Despite focusing on different aspects of language learning, international research has reported positive outcomes in CLIL/EMI settings. Typically, Pessoa et al. (2014) found that the participants developed aspects of their academic writing such as an increased use of academic register, elaboration, and reasoning, and correspondingly less use of descriptive, narrative, informal, and oral-like ways of communicating. Lin and Morrison (2010) revealed that EMI students possessed a wider range of English academic vocabulary and were able to use this more appropriately in their writing for quality academic texts. Tai (2015) concluded that CLIL programmes enhanced students' syntactic accuracy and fluency but not syntactic complexity. These reports align with findings in previous studies that CLIL/EMI has positive impacts on learners' English academic writing. This indicates a lack of evidence on the improvement of the other skills such as reading, speaking, or listening, which might be attributed to a focus on vocabulary and language use in academic writing. However, Yang (2015) reported a significant improvement in learners' receptive skills (listening and reading), and a better performance in productive skills (speaking and writing) compared to other university students in a national-scale English proficiency test. Similarly, Rogier (2012) showed that the participants in his study made considerable gains in all four skills of English in IELTS exams, especially speaking, followed by reading, writing, and then listening. Aguilar and Munoz (2014) revealed that the students made some improvement in listening skills, but their gains in grammar skills were less. This suggests that CLIL may be beneficial for students' English listening competence but not their grammar proficiency (Aguilar \& Muñoz, 2014). These findings have strengthened evidence of the impacts of CLIL/EMI on students' language learning, showing positive outcomes in language skills and vocabulary development.

In general, recent studies have concluded that CLIL/EMI programmes in HE are beneficial for students' language development even if explicit language instruction is limited (Dalton-Puffer, 2008).

\section{Content learning in CLIL/EMI settings}

A number of studies have been conducted to find out the effects of CLIL instruction on content knowledge of learners. Content in CLIL/EMI settings can be "thematic, cross-curricular, interdisciplinary or have a focus on citizenship" (Dalton-Puffer et al., 2010, p. 28). There is a concern reported in studies about how learners' content knowledge, content literacy skills, and understanding of the subject are affected when subject matter is taught in a foreign language (Dalton- 
Puffer, 2011, p. 188). According to Hajer (2000), when learners' language competence is limited, they may not fully understand the subject issues, especially if teachers have to simplify content (p. 264). However, numerous positive results have been reported in primary and secondary education. For example, in 2014, a study was conducted by Dallinger et al. (2016) to investigate both English skills and History knowledge of eighth-graders at 37 secondary schools in Germany. The result showed that CLIL students possessed higher content than non-CLIL students (Dallinger et al., 2016, p. 27).

In $\mathrm{HE}$, research on student content learning is still limited and inconclusive for different reasons. Typically, there is a lack of standardised tests across disciplines to facilitate studies that could yield quantitative empirical evidence (Dalton-Puffer, 2011, p. 188). Some studies, however, have been conducted to find out if English as a language of instruction might have negative effects on student content comprehension and learning at universities. Dafouz et al. (2014) compared and analysed the final grades of EMI and non-EMI students in accounting, finance, and history using Ttests. They found that students in both cohorts achieved similar results in their final performance. In other words, studying in English did not seem to "affect negatively students' academic performance" (Dafouz et al., 2014, p. 232). These results were similar to those of a small-scale study by Tatzl and Messnarz (2013) where experimental tests conducted with 96 engineering students showed no significant differences in the German and English tests. This confirmed that English as a medium of instruction had no impact on student learning of disciplinary content in this context (Tatzl \& Messnarz, 2013). Also looking at the impact of the medium of instruction, Joe and Lee (2013) investigated the lecture comprehension of medical students at a Korean university by comparing the results of the pre-tests and post-tests of students in English-medium and Korean-medium lectures. Similar to the findings of Dafouz et al. (2014), the medium of instruction did not affect students' lecture comprehension. More interestingly, the students' level of understanding in the English lectures did not appear to be determined by their general English proficiency (Joe \& Lee, 2013).

Researchers have suggested different reasons that may contribute to the above findings. One issue is the quality of the tools used to assess students' academic performance as some tests are only able to measure one aspect of disciplinary content (Dafouz et al., 2014) or are not specifically designed for assessing performance, but are instead used for ranking (Tatzl \& Messnarz, 2013). More importantly, the students' English background can be an influential factor, especially when the participants possess advanced levels of English proficiency (Joe \& Lee, 2013) or are supported with an effective ESP programme (Tatzl \& Messnarz, 2013). Regarding this issue, Yang (2015) investigated 
the longitudinal progression of learners in terms of both content and linguistic competence through pre- and post-English proficiency tests for 29 international tourism students in a Taiwanese CLIL degree programme. This was a high CLIL exposure program in which all the courses were instructed in English except for Chinese language and general education courses. The result of the study showed a correlation between content and language learning. The CLIL learners in this study displayed higher levels of content knowledge when they had higher levels of English proficiency (Yang, 2015). This finding raises concerns about the academic success of students who have limited English proficiency in CLIL/EMI programmes, especially when more and more universities have implemented CLIL/EMI for undergraduates regardless of their levels of English.

In summary, although students' limited English might affect their understanding of content knowledge, some studies have shown positive outcomes of content learning, especially at primary and secondary levels. Nonetheless, studies on CLIL/EMI content learning in HE appear to be limited and inconsistent. This may be attributable to the quality of the measurement tools, institutional language support schemes, or students' language background.

\section{Teaching and learning strategies in CLIL/EMI classes}

As a new educational phenomenon in $\mathrm{HE}, \mathrm{CLIL} / \mathrm{EMI}$ has attracted a great deal of attention from educators, researchers, and stakeholders including policy makers, teachers, and students (Macaro et al., 2018). However, there has been little research focusing on the teaching and learning strategies used by the teachers and students in CLIL/EMI classes. Even so, there are studies investigating teacher and student adjustment to CLIL/EMI contexts, which have the potential to provide CLIL/EMI participants with practical experience and implications to implement CLIL/EMI in new contexts.

In Airey's study (2011), the Swedish lecturers reported a number of changes in practice as they began teaching in English. First, preparation for an EMI lesson was longer due to the linguistic demands. Second, the teachers felt they lost depth in their lectures by delivering them in English, and thirdly, the teachers changed their pedagogical style and became less flexible in their lectures. They reported not being confident in expanding the lessons by adding examples, jokes or asides. Fourth, teaching in English impacted the teachers' fluency in both verbal and non-verbal communication. They had high levels of hesitation and false starts, used more filler phrases, and became less "extrovert" in using gestures and body language. Finally, they did not correct students' mistakes in 
English use as they did not consider themselves experts or teachers of English (Airey, 2011), a finding echoed in other studies (Chapple, 2015; Dearden \& Macaro, 2016).

In Korea, Choi (2013) investigated the teaching practices of nine EMI professors at a private university. Participants commented that EMI programmes required new teaching approaches and techniques, which they found challenging. Despite incorporating activities like group discussions, pair work, individual and group presentations, and peer evaluations, the teachers spent $70-80 \%$ of the class time lecturing with or without PowerPoint slides. Some teachers initiated out-of-class activities such as setting up a conference for students, giving feedback on their presentation proposals, or arranging for them to have conversations with international teaching assistants. The teachers also designed activities to assist students' English improvement including summarising class content, incorporating English grammar and pronunciation into content knowledge, or providing tips for English learning (Choi, 2013).

Further examples of language-enhancing teaching strategies have been reported in additional studies exploring CLIL/EMI courses. In Japan, Chapple (2015) found that the EMI teachers employed scaffolding or sheltered content instruction to assist students' comprehension of content knowledge. Scaffolding, introduced by Bruner (1985), is described as a "process that enables a child or novice to solve a problem, carry out a task, or achieve a goal which would be beyond his unassisted efforts" (Wood et al., 1976, p. 90). In this sense, scaffolding strategies are vital to help students identify relevant leaning goals, manage their learning to achieve those goals, and reconcile differences between existing understandings and new concepts to construct new knowledge (Hannafin et al., 2009). More specifically, the teachers in the study said that they provided students with translated documents, simpler explanations, and links to reading in Japanese as well (Chapple, 2015). In China, the teachers commented that they often included illustrative examples from their daily life when explaining complex concepts (Hu \& Lei, 2014). Furthermore, finding their own or their students' levels of English a barrier to teaching subject matter in CLIL/EMI settings, the teachers chose to water down curricular content by simplifying or reducing the required content. They used the language written in the textbook when lecturing and tried to avoid spontaneous interaction and improvisation in class (Hu et al., 2014).

Some common strategies that the teachers used in CLIL/EMI courses were associated with the use of learners' first language (L1). For example, code-switching and translanguaging emerged in many recent studies. Code-switching is defined as a bilingual activity in which more than one 
language, typically the learners' first language (L1) and the target language (L2), are used either intrasententially or intersententially (García, 2009). In Hu et al.'s (2014) study, the teachers of English reported code-switching to Chinese when they had to explain difficult concepts or teach challenging content. Teachers found it hard to communicate effectively when using English to teach complex issues (Hu et al., 2014). This finding resonates with the study of Tarnopolsky and Goodman (2014) who reported that the teachers switched to Russian to explain terminology that caused difficulty in students' understanding of subject matter. Fundamentally, code-switching is used as a strategy supporting interpersonal and interactional purposes in which teachers deliberately use L1 to accommodate to their students' language use (San Isidro \& Lasagabaster, 2019).

Unlike code-switching which suggests that a user moves between two bounded languages, translanguaging captures how users move flexibly across languages which do not operate independently of one another (Lin, 2020). This phenomenon has been recognised relatively recently in multilingual environments and "goes beyond code switching and translation because it refers to the process by which bilingual students perform bilingually in the myriad multimodal ways of classrooms" (García \& Lin, 2019). Students' dynamic bilingual practices can be strengthened by their teachers' pedagogical practices. Teachers adopting translanguaging are able to use students' L1 "as a linguistic resource to facilitate language learning in both English language and content learning" (Liu \& Fang, 2020, p. 1). In their study, Wang and Curdt-Christiansen (2019) reported four different types of translanguaging practices in bilingual university programmes in China. Those types include: (1) bilingual label quest referring to using equivalent labels in L1 and L2 (2) simultaneous code-mixing referring to the simultaneous use of both languages in making meaning, (3) cross-language recapping involving the teaching of the same content in L2 and repeating it in L1, and (4) dual-language substantiation involving "the co-construction of disciplinary knowledge with contributions or insights from both languages" (Wang \& Curdt-Christiansen, 2019, p. 329). Generally, those strategies that enhance translanguaging illustrate how teachers might make use of the L1 for teaching in CLIL/EMI courses, which potentially appropriate for students whose language proficiency is not adequate to follow an English-only manner (Liu \& Fang, 2020).

Students in CLIL classes are also required to use new strategies for learning new content in an additional language. In Yeh's (2014) study, the researcher reported how frequently the students adopted specific strategies in EMI courses. The most commonly used strategies included concentrating in class, taking notes in class, seeking help from peers, and spending more time 
reviewing texts. Few students were found to be using active learning strategies such as forming study groups, asking questions in class, or previewing texts (Yeh, 2014), suggesting that learners were not aware of specific strategies available to them. In Chappel's (2015) research, Japanese students mentioned comparing notes with foreign peers, downloading lecture slides, recording and listening to the lectures again, keeping vocabulary logs and asking Japanese peers as activities to keep up with the EMI classes. This suggests a need for learning strategies to enhance students' language and content learning in CLIL/EMI.

Students also adopted various strategies to overcome unfamiliar language structures in CLIL classes. According to Hu et al. (2014), students in China devised a range of activities they used inside and outside classes including asking teachers to code-switch to Chinese for abstract concepts, referencing Chinese language books, looking up unknown words in the textbooks before class, preparing lessons at home by reading relevant sections, or reviewing slides in line with books written in Chinese after class. Some students even translated the content from English into Chinese, did relevant readings in Chinese, and took notes based on Chinese and English textbooks when preparing for tests (Hu et al., 2014). These findings showed a reliance on the $\mathrm{L} 1$ to deal with the new content language, a finding that aligns with Tarnopolsky and Goodman's (2014) study whereby both the teachers and students in Ukraine "consider the use of the L1 in the classroom to be a natural function of the need for comprehension" (p. 383).

Despite the dearth of research investigating teaching and learning strategies used in CLIL/EMI settings, the few studies available point to the importance of strategies applicable to the dual objectives of CLIL/EMI contexts.

\subsubsection{CLIL and EMI in Vietnamese higher education}

CLIL and EMI programmes are promoted in Vietnamese HE in response to the government's National Foreign Language 2020 project (Tran \& Nguyen, 2018). These programmes aim to reform the HE system by promoting international exchange, enhancing the quality of academic programmes, and preparing a competitive bilingual workforce to access the global job market (Nguyen et al., 2017). More and more universities have implemented different types of EMI programmes in which entire programmes or parts of programmes are taught and learnt in English as a medium of instruction (Tran

\& Nguyen, 2018). These programmes include those founded on formal cooperation with foreign institutions such as Joint Programmes (Chương trình Liên kết) or Advanced Programmes (Chương trình Tiên tiến), and those developed internally such as High-Quality Programmes (Chương trình Đào 
tạo Chất lượng cao) (Nguyen et al., 2017). The introduction of EMI in HE is expected to help students improve their English language proficiency at the same time as they learn content knowledge. Nevertheless, the implementation of the CLIL/EMI policy has created challenges for all stakeholders in HE institutions (Le, 2012; Vu \& Burns, 2014).

In 2012, recognising the lack of scientific research on the implementation of CLIL/EMI in Vietnamese HE, Le (2012) conducted a study investigating EMI policy and practices. He discussed and critiqued a number of issues concerning the policy, teaching and learning, curriculum, and socioeconomic and political impacts that the EMI adoption had created in Vietnam. Finally, he concluded that challenges including the teachers' and students' limited English proficiency, insufficient preparation of teaching and learning materials, curriculum and pedagogy, and insufficient attention to contextual factors would consequently undermine the effectiveness of EMI programmes (Le, 2012). This conclusion is similar to the results reported in Vu and Burn's study (2014). These researchers investigated the perspectives of lecturers participating in an EMI programme in a Vietnamese public university. Analysing data collected from 71 questionnaire respondents and 16 interview participants, they found that the EMI teachers struggled to explain content and answer questions in English and manage students' diverse English abilities and learning styles. The teachers also complained about their lack of pedagogical training and teaching resources in EMI (Vu \& Burns, 2014). In contrast, findings from another study appeared to justify how EMI implementation in Vietnam reflected the impacts of globalisation (Dang et al., 2013). The authors explored the EMI teaching practices of 20 pre-service teachers and findings showed that the participants' teaching decisions were affected by a number of factors. The teachers predominantly used English in class as they wanted to create an English environment for their students. This was also attributed to the social and community pressure that they had from their lecturers, their part-time employers, their students, and social trends, especially the process of internationalisation. In addition, the teachers mainly used authentic audio and video English materials from the Internet rather than printed materials and textbooks because they were aware of the availability of English teaching resources on the Internet (Dang et al., 2013). The results in this study illustrate how these pre-service teachers mediated their teaching practices in EMI programmes to accommodate globalisation in this Vietnamese context. It also showed the positive impact of teacher education in preparing teachers well for the new context (Dang et al., 2013). 
Generally, although the implementation of CLIL/EMI in Vietnamese HE receives support from policy makers, educators and researchers, there remains scant scientific evidence reinforcing its effectiveness, addressing its advantages and drawbacks, and confirming the potential outcomes in language development, content learning, or mobility and employability (Nguyen et al., 2017; Tran \& Nguyen, 2018).

\subsection{Teaching and learning in a digital age}

Education at all levels globally has been responding to the digital age (Saltan et al., 2018). Digital technologies that are introduced into teaching and learning across disciplines include desktop computers, mobile devices, digital recording devices, data logging equipment and associated probes, interactive whiteboards, Web 2.0 technologies and other online resources, and a variety of educational software packages $(\mathrm{Ng}, 2015)$. These educational technologies have the potential to enhance teaching and learning practices (Kalolo, 2019). However, there exist persistent differences "between the well-proven potential of technology enabled learning and the less consistent realities of technology use within university teaching and learning" (Henderson et al., 2017, p. 1567).

In $\mathrm{HE}$, despite the increasing popularity of digital technologies, past research has found little evidence that digital tools have been deliberately integrated into academic activities to transform teaching and learning (Price \& Kirkwood, 2014; Selwyn, 2010). Meanwhile, current research is still discussing the potential and innovations offered by digital technology in teaching, pedagogy, and student learning in HE (Smale, 2017). This section will review literature in the field of education technologies in HE by providing an overview of HE teachers' and students' use of digital technologies in their practices, identifying pedagogical changes as a result of the technological integration, discussing enabling factors for the integration of digital technologies, and highlighting the research on digital technologies in CLIL/EMI contexts.

\subsubsection{Teachers' use of digital technologies}

Teachers are increasingly using digital technologies for multiple educational purposes. These purposes involve the selection of appropriate digital tools to enhance the process of teaching and learning. Teachers use technology for several reasons: to enhance students' academic achievement (Al-Hariri \& Al-Hattami, 2017), to stimulate learning motivation (Liping et al., 2018), to raise the level of student engagement (Bond et al., 2020), to provide collaboration opportunities for students (Mackey, 2015), to facilitate communication between students and lecturer and interaction among students (Froment et al., 2017), and to develop $21^{\text {st }}$ century skills like self-learning (Ferrer-Torregrosa 
et al., 2015), personalised learning (Jensen, 2019), critical thinking and problem-solving skills (Baguma et al., 2019), and digital literacy (Nelson et al., 2019). These multiple purposes have created a need to develop specific teaching strategies for integrating technologies in order to positively influence students' learning (Evans, 2014). In response to this need, there has been an increasing number of studies exploring teachers' use of digital tools.

Teachers use a range of digital technologies to achieve different educational purposes. Shelton (2014) identified two major tools: core technology tools (presentation software and learning management system) and marginal technology tools (web 2.0 technologies, e-portfolio, and eassessment). In their study, Chuang et al. (2015) classified technologies into three groups including Graphical and visualisation tools, ICT tools, and Social media tools. Following such classifications, Cubeles and Riu (2016) specified three categories of technologies that Spanish professors in their study often used: Graphic and visualisation tools, Learning Management Systems tools, and Social media and Mobile tools. Despite identifying various types of technologies, the researchers reported not only a limited use of those technologies by teachers but also some discrepancy in use (Cubeles \& Riu, 2016).

Teachers can utilise digital technologies in a range of ways in their pedagogical approaches. In their review, Kirkwood and Price (2014) analysed 47 articles on technologies used for teaching and learning in $\mathrm{HE}$ and identified three categories in which university teachers use technologies to enhance teaching and learning. These categories include "replicating existing teaching practice, supplementing existing teaching, and transforming teaching and/or learning processes and outcomes" (p. 11). Each category reflects a specific use of digital technologies for educational purposes. While the first two appear to aim at enhancing teachers' existing practices, the third one suggests significant changes in teachers' pedagogical approaches.

In the first category, teachers use technology to replicate an element of traditional teaching and deliver content to students or employ "different technologies for delivering the same material or resources to learners" (Kirkwood \& Price, 2014, p. 13). In other words, technologies are used to conduct existing teaching. Teachers do not change their teaching activities but deploy technologies as a means for delivery. This approach was evident in a number of studies. For example, one teacher in a study had students use a wiki for a joint writing project (Neumann \& Hood, 2009). In another study, the teachers used "synchronous" presentations (e-lectures) and "separate" presentations (i.e. PowerPoint and audio files separately presented) in lecturing (Griffin et al., 2009). In a further study, 
Green et al. (2018) explored the use of YouTube videos as materials for case teaching in health management and policies. They found that YouTube videos can be a "valuable source of content to supplement existing case teaching" (p. 48). This finding aligns with those reported in other research exploring the ways teachers replicate their existing practices with technologies such as the implementation of online courses in comparison to face-to-face courses (Soffer \& Nachmias, 2018), the use of model videos in developing students' oral presentation skills (Okada et al., 2017), and the use of Blackboard Learn in face-to-face courses (Washington, 2019).

In the second category of supplementing existing teaching, teachers provide students with other versions of course materials/resources/tools to support current learning resources or tools. Technologies play the role of assisting tools supplementing teachers' teaching activities. This means that teachers utilise digital tools to create learning support to access and enhance students' learning. Findings from a number of research studies fall into the second category. For example, teachers created podcast episodes supporting each unit so that students could review their lectures (Taylor \& Clark, 2010), or integrated mobile learning activities for students to use with their mobile devices (Wyatt et al., 2010). Chow et al. (2018) investigated the LMS usage of 1457 university teachers and found that $99 \%$ of the teachers used "Content" tools far more than any other tools in the system. This suggests that teachers' use of the LMS was centred on delivering content and "storing learning materials for students to download or access" (p. 133). This result echoes not only those of the studies on the use of the LMS in HE (Garrote Jurado et al., 2014) but those associated with the teachers' employment of technological tools to supplement their teaching practices like using video blogs to optimise student learning outcomes (Liu, 2016), or deploying Facebook and other social media to assist teaching activities (Manca \& Ranieri, 2016).

These two categories recur in HE research (Kirkwood \& Price, 2014). Lecturers commonly use digital technologies as means to conduct and enhance their existing practices. At this level, teachers only change the types of technologies used in their teaching activities but do not change the ways they teach. Tour (2015) found that although teachers perceived different affordances of digital technologies such as offering support and improvement, connectedness, experimentation, sharing, collective intelligence, empowerment, and multimodality, they predominantly utilised technological tools to support existing pedagogies. Tour attributed this to the teachers' thoughts or mindsets regarding the role of digital technologies in teaching and learning, which appeared to have a 
significant impact on their students' experience of learning with digital technologies (Kirkwood \& Price, 2012).

The third category involves transformation whereby teachers use digital technologies to make "structural changes in the teaching and learning processes" (Kirkwood \& Price, 2014, p. 13). Teachers redesign teaching activities or parts of modules to enhance students' learning outcomes. For example, developing blended learning opportunities integrated with online lectures to engage students in active participation (Cooner, 2010). This transformative type of technological intervention occurs rarely in empirical research as teachers tend to use technology to reproduce and reinforce existing practices. Moreover, transformation in teaching and learning can seem complicated and requires a lot from teachers in selecting pedagogical approaches (McKnight et al., 2016).

Although university teachers can easily access a wide range of digital technologies, their actual use of those digital tools and commodities has been limited. In their study, Marcelo et al. (2015) investigated how 941 lecturers in ten Spanish universities employed technologies in their teaching activities. They found that more than $50 \%$ of the participating lecturers rarely used technology and the type of activities that they used technologies for were very limited. This raises questions about how commonly teachers use technologies to make significant changes to their teaching practices and if so, what changes they make. My study will focus on deepening understandings of such pedagogical changes within its focus on lecturers' use of digital technologies in the novel context of Vietnamese EMI.

\subsubsection{Pedagogical changes}

Even though there is little evidence reporting lecturers' transformative use of digital technologies for teaching, the introduction of digital technologies for educational purposes has gradually led to some visible changes in teachers' pedagogical practices. In other words, teachers are starting to "think of new ways to provide teaching and learning opportunities" to integrate technologies (Angela \& Alejandra, 2018, p. 1335). The digital environment prompts teachers to make decisions about how they will integrate technologies into their teaching based on their view of pedagogical strategies that might enhance student achievement. For example, in the study by Marcelo et al. (2015) on university teachers' levels of technology usage, a significant group of lecturers frequently employed digital technologies "as a support to develop a more ample variety of learning activities for their students" (p. 123). This suggests that the perceived affordances of digital 
technologies might have been a driving force for changes to their teaching practices. A number of pedagogical changes have been observed in research on teaching with digital technologies (Englund et al., 2017; Kalolo, 2019; Koh, 2019).

Technology has the potential to change the roles of teachers and students in teaching and learning (McKnight et al., 2016). McKnight et al.'s study found that students with access to multiple digital resources became less reliant on the teacher, and at the same time the teacher took on the role of guiding and facilitating students in their learning. In his Integrative Research Review of the digital revolution and its impact on education systems in developing countries, Kalolo (2019) found that digital technologies can transform approaches to constructivist learning and allow the pedagogical roles of students and teachers to be redefined. While students are encouraged to engage and invest more into their learning, teachers reduce their overt control of knowledge dissemination. This shift, together with the implementation of technology allowed the teachers in these studies to restructure their time and spend more time on "side-by-side coaching, one-on-one support, and providing immediate feedback to the students in the classroom" rather than "providing whole-class instruction and grading papers, assignments, and tests, as well as tracking, reporting, and locating late or missing student work" (McKnight et al., 2016, p. 204). In other words, appropriate use of digital technologies appears to offer teachers affordances to personalise learning for their students and focus on their needs. This highlights that integrating technology into their practice may have benefits for teaching and learning processes.

One potential change is a shift from teacher-centred to student-centred approaches in teaching with technology. A student-centred approach is defined as "ways of thinking about teaching and learning that emphasise student responsibility and activity in learning rather than content or what the teachers are doing" (Newble, 1989, p. 16). This approach, derived from constructivism, emphasises the role of students in the learning process as active constructors of their own skills and knowledge (Tursunov, 2016). A student-centred approach is believed to benefit students in developing autonomy and independence and reflects a global interest in developing citizens with high order thinking, and skills in working cooperatively and autonomously (Nurjannah et al., 2017; Pham, 2011). When technology is introduced, teachers may engage students in the co-construction of knowledge which is referred as the process in which students work and negotiate meanings with their peers under the guidance of their teachers to construct new knowledge (Ahn \& Class, 2011). In their longitudinal study, Englund et al. (2017) explored changes in conceptions and approaches to teaching 
with technology of nine teachers at a Swedish university. They found that novice teachers showed a rapid change from teacher-focused to more student-focused approaches. Specifically, those teachers used virtual simulation to create a learning environment where students could communicate, collaborate and co-construct knowledge. In this study, although a few experienced teachers encountered challenges teaching with technologies, they managed to adopt approaches that considered students' learning needs. This is consistent with other research that connect the application of technology to a more student-centred approach (Smirnova et al., 2019).

$\mathrm{Ng}$ (2015) reminds us that transformative pedagogies only evolve when teachers integrate technology "purposefully, thoughtfully and on a regular basis in their classroom practice" (p. 17). In a literature review of 56 cases of flipped classrooms, Koh (2019) revealed that "flipped classrooms personalise learning through resource and teacher access, develop higher order thinking through problem-solving, and engage students in collaborative learning through both peer groups and design groups" (p. 14). In other words, the pedagogy reflected through the teachers' flipped classroom practices can positively impact students' learning. In this case, flipped classrooms are viewed as "the instructional practice where lecture content is made available online for pre-class study and in-class learning activities are used to deepen content understanding" (Koh, 2019, p. 15). Similar findings were reported in studies in which teachers used smartphones or mobile learning to enhance student learning through collaboration (Farley et al., 2015), problem-solving activities (Kim et al., 2011), or self-directed learning (Lötter \& Jacobs, 2020). This illustrates substantial changes in teaching practices when teachers deliberately use digital technologies for pedagogical purposes (Lötter \& Jacobs, 2020).

However, research also suggests that the teachers' use of digital technologies in their classes was not only infrequent and isolated, but also basic and used predominantly for administrative purposes. For example, from their 2012-2016 surveys of technology-enhanced learning for HE in the UK, Walker et al. (2018) found that while educational institutions had invested heavily in providing more technological tools and devices for teachers and students, there were few changes in pedagogical practices. This calls into questions the influences on teachers' decisions and ways of adopting digital technologies into their teaching activities. My research will investigate how the teachers in EMI courses made pedagogical decisions to include technologies into teaching subject matter through English. 


\subsubsection{Students' use of digital technologies}

Studies have reported different aspects of students' technology use, such as their patterns of usage (Lai \& Hong, 2015; Sumuer, 2018), perceptions of usefulness (Henderson et al., 2017), preparation for e-learning (Parkes et al., 2015b), digital literacy ( $\mathrm{Ng}, 2012$ ), use of digital and social media (Bond et al., 2018), and their negative engagements with digital technologies (Selwyn, 2016).

Research shows that students perceive and utilise technological tools in different ways in their learning. Henderson et al. (2015) surveyed 1,658 students in two Australian universities to explore their patterns of technology use. They concluded that digital technologies were an essential part of university study as the students used various digital tools for different activities in their learning. These activities included accessing "official" digital resources such as e-books or e-textbooks through the LMS and online library resources, searching for "non-official" materials using Internet search engines like Google, engaging with visual materials such as videos on video-sharing websites like YouTube, and communicating and collaborating with peers and teachers on Facebook (p. 311). These findings are consistent with the results reported in Margaryan et al.'s (2011) study which showed that the university student participants predominantly accessed course information and materials such as e-books, lecture notes and announcements via virtual learning environments. Participants also communicated and collaborated with friends to discuss assignments or prepare for exams using mobile phones or social networks, and, most commonly, they viewed YouTube video clips for both informal learning and entertainment. These findings are echoed in other studies (Bond et al., 2018; Henderson et al., 2017; Parkes et al., 2015b).

Research has revealed mixed results about the effect of students' use of technology in HE. While students are likely to enhance their learning through sharing resources and collaborating with others in the learning networks (Sweeney, 2017), they mainly employ digital technologies for basic tasks. Bond et al. (2018) surveyed 1,327 students in Germany and found that more than $80 \%$ of the participants frequently used search engines, the LMS, computers outside of the university, and email accounts for studying. This finding mirrors the results of other studies that have found that students' use of technology is predominantly for logistical purposes in learning. For example, Henderson et al. (2017) reported that the majority of students in their study $(n=1,658)$ used technology to organise

and manage "the logistics of studying such as managing schedules, timetables, fulfilling deadlines and course requirements, and 'keeping in the loop' with regards to university news and course information" (p. 1571). Similarly, Parkes et al. (2015b) found that the highest digital competence 
demonstrated by the students in their study was in downloading and uploading information and resources, responding to others, and seeking information through either own enquiries or the questioning of others.

Moreover, the range of technologies and the extent to which students used them were also limited. In their study, Lai and Hong (2015) explored the digital technologies used by 880 students at one university in New Zealand. They found that two thirds of the participants "only used digital technologies up to 10 hours per week on university work and for social and personal activities respectively" and the tools they commonly used were "laptop computers, Internet website, Google, MP3/iPod, Facebook/MySpace and mobile phones" (p. 735). This finding aligns with those reported in other research (Newman \& Beetham, 2017). This might be explained by the students having little understanding of how digital technologies may support learning (Margaryan et al., 2011) and still requesting explicit help and support from teachers (Sumuer, 2018). This suggests that the purposes for students' use of technologies in learning are in line with their teachers' teaching practices with technologies. When lecturers rarely change their pedagogical style with digital technologies, students tend to respond by being reluctant to change their learning. My study will focus on how students deployed digital technologies in their EMI courses in response to teachers' teaching practices and the impact of the new context.

\subsubsection{Factors influencing the integration of digital technologies}

Despite the increasing use of digital technologies in HE contexts (Berrett et al., 2012), the uptake and integration of technology in teaching and learning appear to be patchy and even limited (Tondeur et al., 2017). Recent research has reported that undergraduate students apply digital technologies unevenly in terms of frequency of technology use, types of technologies adopted, and their readiness to integrate technology into learning (Clark-Gordon et al., 2019). Even taking into account the findings from these studies, the influences on students' choices to assimilate digital technologies into their learning remain unclear. A similar question can be asked of their teachers' decisions in adopting technologies into their teaching practices (Kreijns et al., 2013). Studies have reported that teachers have made few and slow changes to their pedagogical practices to include digital technologies even though they perceive the potential of digital technologies in enhancing students' learning $(\mathrm{Ng}, 2015)$. The next section discusses relevant literature addressing the influences affecting how teachers and students integrate digital technologies in HE contexts. 


\subsubsection{Facilitating conditions}

Facilitating conditions refers to contextual factors exerting an influence over teachers' and students' decisions to perform a task using digital technologies (Teo, 2010). These factors might involve institutional policy, accessibility and availability of infrastructure, or administrative and technical support. They play a significant role in enabling or hindering the use of digital technologies in teaching and learning. In the model of Unified theory of acceptance and use of technology (UTAUT) (Venkatesh et al., 2003), facilitating conditions are identified as an environmental factor affecting the acceptance and use of a specific information technology in academic activities. Research examining the application of UTAUT in HE has reported how and why teachers and students have conducted technological integration in their practices (Chu \& Chen, 2016). This influence was evident in Ángel et al.'s (2019) survey of 370 Spanish university students' use smartphones and tablets as resources for learning. They found that facilitating conditions was an influential construct in students' learning with technological devices. In this context, the students not only valued the institutional assistance and technical support, but also required specific guidance on how to resolve problems and incidents. Another study conducted by Jung and Lee (2015) explored factors predicting teachers' and students' use of YouTube videos in Japan and the USA. The results showed that facilitating conditions was one of the most influential predictors of their decisions to use YouTube clips in teaching activities. These examples indicate that educational contexts play an overarching role in facilitating or impeding teachers' and students' integration of digital technologies into their academic practices.

Institutional technology policies appear to exert a strong influence on the adoption of digital technologies in academic activities. Such policies are usually included in institutions' mission and vision statements, or the strategic plan where the universities state how and why technologies should be used to enhance teaching and learning. With a clear vision of the important role of technology, policy makers are able to require teachers and students to make innovations by supporting them with sufficient resources. In their study, Czerniewicz and Brown (2009) investigated the relationship between university policy and the e-learning use through a survey of 3533 students and 216 teachers from six diverse South African universities. They found that the e-learning policies at the institutions were enacted through resources and systems which guaranteed access to appropriate technologies and enabled teachers and students to use ICT frequently. In other words, when the institutional policy determined the goals, values, and resources for digital technologies, this "appear[ed] to provide a 
generally more enabling context environment making it possible for ICTs to be used" (Czerniewicz \& Brown, 2009, p. 130).

The accessibility and availability of infrastructure for digital tools is another consideration affecting teachers and students in deciding whether and how to use digital technologies. Lack of access to appropriate technologies might not only hinder teachers and students from adopting technology, but also restrict the ways they use technologies for educational purposes (HamiltonEkeke \& Mbachu, 2015). For example, teachers reported that the lack of infrastructure support and access was the main constraint impeding their use of the e-learning platform of Educational Support System in Akinde and Adetimirin's (2019) study. Conversely, in Porter and Graham's (2016) study, access to adequate infrastructure had significant impacts on the teachers' decisions to adopt blended learning. These findings emphasise the importance of institutions providing teachers and students with technological tools and support if they are expected to integrate digital technologies into their educational contexts.

\subsubsection{Beliefs}

Beliefs are an influential factor in many areas of education including the integration of digital technology (Galvis, 2012). Research has reported how teachers' and students' beliefs affect their decisions about using technologies. Teacher beliefs defined as a system of multiple and interconnected beliefs that encompass knowledge construction, learning and teaching may include teachers' opinions about "how students learn, what a teacher should or should not do, and which instructional strategies work effectively" (Kim et al., 2013, p. 199). In other words, teacher beliefs impact on teachers' behaviours in class ( $\mathrm{Ng}$ et al., 2010) and shape their teaching practices (Song \& Looi, 2012). Similarly, teachers' beliefs about technology are likely to predict the ways in which they integrate technological tools and products into their teaching activities (Miranda \& Russell, 2012). In a similar way, students' beliefs or perceptions about technology are likely to inform how and why they use digital resources in their learning (Henderson et al., 2017). This section addresses different aspects associated with teachers' and students' beliefs or perceptions about digital technologies including teachers' pedagogical and disciplinary beliefs, students' perceptions of the usefulness of technology, and teachers and students' self-efficacy.

Teachers' disciplinary beliefs can be understood as the teachers' beliefs about the nature of content knowledge and how best to teach it (Gleeson \& Davison, 2016). Teachers in a subject community share beliefs and understandings of the knowledge in the subject and know how to select 
appropriate teaching practices for learners (Darling-Hammond et al., 2005). It is therefore useful to identify such disciplinary thinking within particular subjects that underpins the distinctive pedagogical practices required for those subjects. Teaching professionals need an understanding of both content and process, including content knowledge, pedagogical knowledge and curriculum knowledge (Shulman, 2013). The relationship among these types of knowledge reflects content teachers' beliefs and influences their teaching approaches (Gleeson, 2010). This suggests that the pedagogical decisions that subject teachers make related to the use of digital technologies to teach content knowledge are potentially impacted by their disciplinary beliefs.

Teachers' pedagogical beliefs have been described as an overarching factor affecting the teachers' use of digital technologies in teaching (Tondeur et al., 2017). Teachers' pedagogical beliefs are their beliefs about teaching and learning that encompass a complex and multifaceted structure of their understanding, premises, or propositions about teaching and learning (Ertmer \& OttenbreitLeftwich, 2010). In other words, pedagogical beliefs serve as a guide for teachers to make decisions in their teaching. As such, teachers' pedagogical beliefs have the capacity to influence their practices using digital technologies. In a literature review on the relationship between teachers' pedagogical beliefs and technology use in education, Tondeur et al. (2017) reported key findings describing the relationship between pedagogical beliefs and technology use, the types of technology use, and the role of beliefs in teachers' professional development. This indicates that teachers' pedagogical beliefs may significantly affect how and why teachers use digital technologies in the process of teaching and learning.

Pedagogical beliefs include ideas about teacher-centred and student-centred practices (Deng et al., 2014). While teacher-centred practices are related to behaviourism and may focus on discipline, subject matter, and moral standards, student-centred practices are associated with constructivism and/or social constructivism emphasizing individual student needs and interests (Deng et al., 2014). In the field of educational technologies, teachers possessing constructivist or student-centred beliefs tend to express positive attitudes towards the use of digital technologies and proactively use them to support students' capacity in learning (Ertmer et al., 2014). For example, Liu et al. (2017) surveyed 202 foreign language teachers at seven Chinese universities and found that “the participants' constructivist pedagogical beliefs had a positive influence on their perceptions of ICT as being easy to use, as being useful" (Liu et al., 2017, p. 757). This suggests that teachers holding constructivist beliefs are likely to be open to integrating technology into their professional practice. 
The findings are similar to Englund et al.'s (2017) study which reported that the teachers who held student-centred conceptions more readily adopted technology that supported student-centred approaches. In particular, those lecturers with student-centred conceptions reported using communication technologies to create digital simulations for students, facilitating students to collaborate and communicate with each other in a virtual platform, or supporting students to create their own digital learning resources (Englund et al., 2017). These findings are consistent with other studies (Gupta \& Dharamveer, 2017; Teo et al., 2019) which also identified the impacts of teachers' pedagogical beliefs on their teacher-centred or student-centred practices in teaching with digital technologies.

Teachers may be encouraged to integrate technology into their teaching if using digital technologies aligns with their beliefs (Voogt et al., 2013). It then follows that teachers holding traditional teacher-dominant pedagogical beliefs are likely to be reluctant to use digital technologies in their teaching activities. Such teachers tend to use technologies in traditional ways for simple tasks such as word processing and presentations rather than trying active, meaningful, and innovative uses of technology. In their study, Liu et al. (2017) found that "while teachers may have positive attitudes toward ICT, their use of it might still be restrained due to their traditional transmissive beliefs" ( $p$. 760). This finding is consistent with what Jääskelä et al. (2017) reported in their study. The teacher participants not only concerned about having to change their practices and get more work but also doubted "'the applicability of technological tools to their subject teaching or their own/their students' digital competences" (p. 24). In other words, the teacher's beliefs about pedagogy appeared to affect their desire to include digital technologies in their teaching practice. Similarly, Taimalu and Luik (2019) revealed that while constructivist pedagogical beliefs seemed to have a positive effect on the teachers' beliefs about the value of technology use, traditional pedagogical beliefs seemed to have a negative influence. These relationships were illustrated by differences in the ways in which an extent to which the educators adopted digital technologies (Taimalu \& Luik, 2019). These studies indicate the critical role of teachers' pedagogical beliefs in decisions around using technologies for teaching.

Teachers' practices are not only shaped by their beliefs, but also by their sense of self-efficacy. Self-efficacy refers to people's beliefs in their capability to "to organize and execute the course of action required to manage prospective situations" (Bandura, 1986, p. 2). Such beliefs regulate people's choices, effort, and persistence in dealing with obstacles as those people reflect their 
personal experience and make decisions about future potential actions (Bray-Clark \& Bates, 2003). Teacher self-efficacy refers to the degree of confidence that a teacher has in their ability to promote students' learning (Bandura, 2010). Teachers with different levels of self-efficacy tend to conduct their teaching practices in different ways. For example, those with high self-efficacy are likely to engage students in different modes including working individually, in pairs, and in groups (Smylie, 1989); efficacious teachers may also prefer working with students in small groups and use more individualised instruction (Tschannen-Moran, 2001) as opposed to whole class instruction.

Consistent with findings about teachers' beliefs, students' perceptions on the usefulness of technology appear to affect how and why they use technological tools in their learning. Although students increasingly adopt a wide range of digital technologies for learning, the actual effect of their technology use on learning seems debatable. This limited uptake of technologies for learning may arise from their perceptions of the potential offered by various technologies to enhance their academic activities. Henderson et al. (2017) reported that the students in their study made decisions to use digital tools when they considered such technologies as having 'educational' benefits for their learning (p. 1567). This is consistent with the result of a study by Bond et al. (2018) who found that the students predominantly used passive forms of technology such as word processing software, lecture recordings and search engines which they considered to be useful for learning. This is one of many studies reporting students' low-level use of collaborative and creative digital tools such as blogs, micro-blogging, social networks for interacting with others in learning communities, and commenting upon or critiquing lecturer responses in forums due to their perception of usefulness (Bond et al., 2018; Parkes et al., 2015a). These findings suggest that students' adoption of digital technologies for learning depends on their perceptions of whether technologies are likely to be beneficial in supporting their academic studies.

Moreover, students' self-efficacy plays a significant role in their selection of learning strategies. Research has found that students possessing significant or increased levels of self-efficacy can experience many benefits in their learning. These benefits might include:

a firmer commitment to given undertakings, an openness to unfamiliar experiences, an increased emotional stability amid stress, a stronger social proactivity in interpersonal relationship, an elevated sense of vision on goal setting, a more vigorous effort to reach higher goals, and an increase in motivation. (Cave et al., 2017, p. 84).

Student self-efficacy is defined as the students' beliefs about their own capability in performing specific tasks to accomplish desired goals and produce certain outcomes (Bandura, 2010). That is why 
self-efficacy is considered to be the strongest predictor of behavioural changes in any human endeavour (Richardson et al., 2012). In other words, students with different levels of self-efficacy might look for particular learning strategies to achieve their objectives, which leads to their decisions of whether and how they use digital technologies in their learning.

\subsubsection{Knowledge and skills.}

Knowledge and skills related to digital technologies could be considered as influential factors in the technology integration of teachers and students (Eickelmann, 2011). Teachers and students are more likely to use technological tools and products to support their practices if they feel confident of their knowledge and skills. In contrast, they are unlikely to use digital technologies in teaching and learning if they have not learnt how to do so. This part will review literature on how teachers' knowledge and students' skills affect their engagement with technologies for educational purposes.

Teacher knowledge impacts on instructional practices (Loewenberg-Ball et al., 2008). In the 1980s, Shulman (2013) researched the knowledge of teachers and suggested a framework for teacher education in which he distinguished three categories of content knowledge including: subject matter content knowledge, pedagogical content knowledge, and curricular knowledge. These types of knowledge, especially content knowledge and pedagogical content knowledge, have been shown to influence not only teachers' instructional practices but also student learning (Baumert et al., 2010). Content knowledge or subject matter knowledge provides teachers with thorough understandings of how to organise key concepts in their disciplines (Shulman, 2013). Disciplinary teachers need to comprehend not only how the concepts and principles of the discipline are organised but also how accepted truth in the disciplines is established. In addition, teachers with strong content knowledge are also able to "understand why a given topic is particularly central to a discipline whereas another may be somewhat peripheral" (Shulman, 2013, p. 6). Although content knowledge differs from specialised knowledge about how to teach content, thorough understanding of the content enables teachers to communicate the subject matter flexibly in multifaceted ways (Shulman, 1987). As such, teachers' subject matter content knowledge will be salient in teachers' process of making pedagogical decisions in their teaching. Pedagogical content knowledge is defined as subject matter knowledge for teaching that encompasses "the ways of presenting and formulating the subject that make it comprehensible to others" (Shulman, 2013, p. 6). Teachers with strong pedagogical content knowledge can access various forms of subject matter representation by including powerful analogies, illustrations, examples, explanations, and demonstrations in their teaching practices. 
Teachers need knowledge about technological tools and devices to use them for teaching purposes (Spiteri, 2020). Specifically, they require knowledge about technology and pedagogy which is applicable to the subjects they teach. To conceptualise this knowledge, Mishra and Koehler (2006) introduced the Technology, Pedagogy, And Content Knowledge - TPACK- framework which was built on Shulman (1986) notion of Pedagogical Content Knowledge (PCK). The TPACK framework combines three main constructs of teachers' knowledge: technology, content, and pedagogy, and describes “how teachers' understanding of educational technologies and PCK interact with one another to produce effective teaching with technology" (Harris et al., 2009, p. 62). In this framework, equal importance is paid both to the relationships of the major constructs mentioned above and to the interaction of three other bodies of knowledge encompassing pedagogical content knowledge (PCK), technological content knowledge (TCK) and technology pedagogical knowledge (TPK) (see Figure 1). As digital technologies enter HE, TPACK has become a model that can be used to investigate of use of technology in pedagogical practice in higher education as well as in schools (Matt \& Orna, 2017).

\section{Figure 1}

The TPACK framework and its components (Harris et al., 2009, p. 396) adapted from (Mishra \& Koehler, 2006)

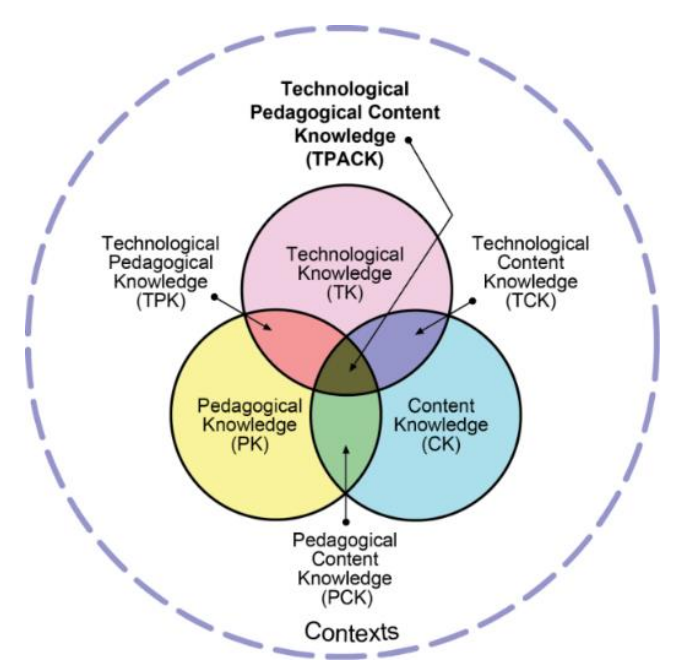

Teacher knowledge (TPACK) may influence decisions to deploy digital technologies in teaching and appears to be connected with their beliefs or attitudes towards the use of digital technologies in their classrooms. Further, teachers' TPACK is likely to affect their self-efficacy, and their perceived usefulness and ease of use of technologies, which affects their decisions to use digital technologies in academic activities (Yang et al., 2019). Hsu (2016) examined 158 Taiwanese English teachers' TPACK and how it affects their adoption of mobile-assisted language learning. He found 
that the teachers' TPACK significantly affected their acceptance of using mobile devices in language teaching and learning. The teachers who possessed high levels of TPACK demonstrated high levels of self-efficacy in teaching. This is consistent with what Yusop and Razak (2020) reported in their study that the teachers' level of TPACK emerged as the strongest predictor in determining the use of ICT in their teaching practices. This suggests that teachers' knowledge has a strong influence on their integration of digital technologies into teaching. The TPACK framework has been used to explore teachers' use of technologies for teaching. For example, Vasodavan et al. (2019) found that lecturers' technological knowledge encouraged them to use different types of technology but did not guarantee successful collaborative learning. In contrast, Matt and Orna (2017) identified that the lecturers in their study displayed not only limited interest in integrating educational technologies, but also limited technological knowledge in how they used technology for teaching. These limitations appeared to have reduced the lecturers' engagement with technology and inhibited opportunities to use technology in their teaching. The findings from these studies suggest a relationship between teachers' technological knowledge and whether or how they used technologies in their teaching.

Researchers have debated this framework. While researchers know that TPCK is derived from Shulman's PCK, they do not agree on what PCK entails. This explains why the definition of TPACK is still contested. Voogt et al. (2013) supported the view of TPACK as a distinct body of knowledge as PCK is considered as a domain of knowledge related to the understanding of students' learning difficulties and perceptions when teaching particular topics in that domain. As such, they called for a need to discuss how TPACK applies within specific subject domains and how technology can be used to support student learning in that domain. Moreover, researchers report that TPACK as a knowledge base has close connections with teachers' beliefs, which may play a role in teachers' use of technology for teaching (Valtonena et al., 2006). This suggests that it is necessary to "understand how a teacher's technological reasoning affects his (or her) decision making while using technology" (Voogt et al., 2013, p. 119).

Technological skills are crucial for teachers and students to optimise the educational benefits of digital technologies. Studies report that lack of digital skills is likely to restrict teachers and students' use of technologies to transform teaching and learning (Bond et al., 2018; Parkes et al., 2015a). Bond et al. (2018) found that the teachers did not perceive the usefulness of the institutional tools because they were not aware of the availability of those tools nor used them before. In this case, the teachers' lack of skill in using those technologies made them reluctant to integrate the tools 
into their practices. Similar findings identified students who mainly used digital tools for daily activities such as searching and email did not know how to deploy technologies for interactive and collaborative tasks (Bond et al., 2018). These findings echo those reported in a study by Parkes et al. (2015a) revealed that the expressed low levels of preparedness for competencies associated with interactions with content, collaborating with others, and "balancing work, social, family and study lives in an e-learning environment" (p. 7). In summary, limited digital skills and competence are likely to hinder teachers and students from integrating digital technologies effectively into their teaching and learning practices (Marcelo \& Yot-Domínguez, 2019). This implies that teachers may need to gain sufficient technological skills through professional development in order to provide students with appropriate guidance and support to use technologies for learning (Englund et al., 2017).

\subsubsection{Digital technologies in CLIL/EMI contexts}

In the context of CLIL/EMI, teachers and students use English as a medium of instruction for the teaching and learning of subject matter. In the field of educational technologies, while there have been studies exploring the integration of digital technologies in English teaching and learning, there has been scant research investigating the technology use in CLIL/EMI contexts. In this section, I will first discuss the impact of digital technologies on English teaching and learning, and then review studies on the use of digital technologies in CLIL/EMI context.

\subsubsection{Digital technologies in language teaching and learning.}

In recent decades, the use of digital technologies in the field of language teaching and learning has sparked much research interest (Rustam \& Mengke, 2020). Research has reported various types of technologies used to assist language teaching and learning such as games (Chen et al., 2019), corpora (Frankenberg-Garcia et al., 2019), automated feedback (Ranalli, 2018), social networking (Maier et al., 2015), virtual reality (Hassani et al., 2016), websites and digital resources (Shen et al., 2015). These technologies aimed to improve different language skills including writing, vocabulary, speaking, reading, listening, grammar and phrases (Rustam \& Mengke, 2020). Overall findings from these studies have supported the positive impact of digital technologies on language learning and instruction (Golonka et al., 2014; Rustam \& Mengke, 2020).

A range of digital technologies has been used for foreign language teaching and learning. As technologies have become well-established, accessible and available in both social and academic domains, teachers and students have incorporated them for different purposes in their practices. Reviewing 350 studies on the use of technologies in foreign language teaching and learning, Golonka 
et al. (2014) reported various types of digital technologies that have been employed by teachers and students. These technologies were categorised into different groups including schoolhouse or classroom-based technologies, individual study tools, network-based social computing technologies, and mobile/portable networkable devices. The researchers also identified affordances of these technologies for language study such as facilitating course content organisation, and teacher-student and student-student communication, incorporating authentic content into lessons, supporting learner autonomy, tailoring instruction to individual learners, encouraging collaborative learning, or enabling personalised learning. The overall results show "moderate support for claims that technology enhanced learners' output and interaction, affect and motivation, feedback, and metalinguistic knowledge" (Golonka et al., 2014, p. 70). This suggests that technology can have a positive influence on foreign language teaching and learning.

The integration of digital technologies appears to increase the development of students' English proficiency. Research has reported positive findings associated with the teachers' use of technologies to enhance students' English skills (Won \& Kim, 2018). In a review of literature, Turan and Akdag-Cimen (2019) found that the flipped classroom method was commonly used to enhance different proficiency skills, with evidence that speaking skills benefited from digital technologies. For example, Köroglu and Çakir (2017) investigated the effects of flipped instruction on the speaking skill development of 48 students in experimental and control groups. They found that flipped instruction and flipped classrooms can improve language learners' fluency and coherence, lexical resource, grammatical range and accuracy, and pronunciation (Köroglu \& Çakir, 2017, p. 52). This finding is consistent with other research which has reported the effects of digital technologies on the improvement of discrete skills such as speaking (Chen Hsieh et al., 2017), listening (Ahmad, 2016; Al-Baekani \& Ridwan, 2018), writing (Kongsuebchart \& Suppasetseree, 2018), and reading (Bataineh \& Mayyas, 2017).

Additionally, there is evidence that students' vocabulary and grammar are enhanced through the use of technologies (Persson \& Nouri, 2018). For example, Ali and Ahmad (2017) explored the impact of a model using computers, mobile phones, and WhatsApp on the vocabulary acquisition of 122 students at a public university in Saudi Arabia. They found that students using the vocabulary learning model improved significantly in their vocabulary acquisition. Similarly, a positive result in students' grammar learning through the use of technologies was reported in Kılıçkaya's (2015) study. Kılıçkaya (2015) examined the effects of computer-based instruction on the achievements of 50 
English learners and reported that the participants who received both computer-based and teacherdriven grammar instruction supported by computer-based materials got higher scores than those receiving traditional instruction" (Kılıçkaya, 2015). In short, these studies suggest the potential of using digital technologies to enhance students' English proficiency.

Digital technologies have been found to affect different aspects of English teaching and learning processes. Particularly, technology has enhanced students' engagement and motivation in learning (Fisher et al., 2018), it has facilitated students' collaboration and interaction (Wu, 2018), and stimulated their autonomy and personalised learning (Tsai, 2019). For example, Sarhandi et al. (2017) studied the impacts of smartphone based activities on the engagement of 50 university EFL students at intermediate level and found that the students using smartphones for learning were more engaged than those in the control group. Specifically, they had "significantly faster initiation times, and were significantly less distracted" (Sarhandi et al., 2017, p. 113). Another example is the study conducted by Tsai (2019) who reported "significant correlations between online learning activities and perceived learner autonomy"; in other words, the students in the experimental group demonstrated improved levels of autonomy regarding "strategy use, behaviour, interaction with the materials, the use of social resources and self-management of learning" (Tsai, 2019, p. 1). These findings add further support to the benefits of including digital technologies in the field of English teaching and learning.

Research has revealed the changing nature of digital technologies in language teaching and learning. Reviewing articles from 2014 to 2019, Rustam and Mengke (2020) found that there was a change in the types of technologies used in language classrooms, particularly with the implementation of online videos, e-books, augmented reality, and wearable devices which had not been used before 2014. This has contributed to a steep learning curve for language teachers who may not have kept pace with the theoretical and practical knowledge required to adopt new technologies in their field. There is also a change in research focus associated with the effectiveness of technologies for developing language output rather than language input which was emphasised in traditional classrooms. Moreover, scholars in technology-enhanced language education have begun to address issues related to increasing students' proficiency when learning with technology (Türk \& Erçetin, 2014), understanding their learning strategies (Ziegler, 2016), the technical problems they may have (Shadiev et al., 2018), and negative effects of using technologies on their learning and wellbeing (Zhonggen, 2018). There is limited research exploring the use of technologies in content teaching and learning in language education. While early studies focused on the use of digital 
technologies supporting discrete skills in language learning, which reflects a more traditional approach to learning an additional language, there is a need for research on digital approaches that enhance language education holistically. My study will address these gaps when investigating the EMI context.

\subsubsection{Digital technologies and CLIL/EMI practices.}

The increasing popularity of CLIL/EMI and the evolution of digital technologies are considered to be key issues affecting HE in today's globalised academic world (Querol-Julián \& Camiciottoli, 2019). In the process of teaching and learning, both CLIL/EMI and technology integration appear to influence learning. Despite its rapid development, EMI has created significant challenges for teachers and students who are not native speakers of English and researchers have investigated whether and how digital technologies might contribute to CLIL/EMI practices.

One of the early studies discussing the use of technology in CLIL/EMI was conducted by Gimeno et al. (2010). These researchers recommended InGenio, a web-delivered authoring tool used for CLIL teachers to create and share materials and tasks, and for students to access materials and learning activities via the Learning Environment platform. Although they did not investigate the effects of the technological product on CLIL, the authors suggested the possibilities of technological tools and digital platforms for supporting teachers and students in CLIL contexts.

There is empirical evidence supporting the integration of digital technologies in CLIL/EMI settings. For example, in their study, Paliwoda-Pękosz and Stal (2015) reported that most students were satisfied with the range of Moodle tools in the Virtual Learning Environment system and perceived it useful in providing students with more interactive resources for learning content and creating a collaborative environment where students can share their experience, find support, and interact with friends and teachers. The authors also suggested a framework of blended learning using technologies to enhance the effectiveness of CLIL courses. The use of VLE in blended learning or mobile learning was presented as "an effective approach that improves lecture comprehension, encourages more class engagement, promotes collaborative learning, and achieves better learning outcomes" (Chuang, 2017, p. 640). These studies reported positive impacts from using various technologies on CLIL/EMI courses. There are concerns, however, about the trustworthiness of assessing and evaluating the effectiveness of these technologies in CLIL/EMI settings as there appears to be little evidence reporting the use of measurement tools for assessment and evaluation in these studies. 
Recent research focuses on pedagogical approaches which combine a range of digital technologies in CLIL/EMI classes, with mixed results. Flipped classroom pedagogy has been investigated to see whether it enhances teaching and learning practices. For instance, Choi et al. (2015) surveyed 75 students in an EMI nursing course using the flipped learning model and revealed that the students preferred the flipped learning strategy as this method helped enhance their understanding of lecture content. This finding appears to be inconsistent with what Karjanto and Simon (2018) reported in their study where the students perceived an improvement in communication and engagement in the course but they still struggled to understand the materials and content (Karjanto \& Simon, 2018). This points to a need for further investigation.

There are limited studies researching the adoption of digital technologies in CLIL/EMI teaching and learning. My study will focus on this gap by exploring how and why the teachers and students deployed digital technologies in the new context of EMI in Vietnam.

\subsection{Conceptual framework: ROAD-MAPPING}

This section discusses in detail the conceptual framework underpinning the interpretation of the study findings. The ROAD-MAPPING framework was developed to examine English-Medium Education in Multilingual University Settings (EMEMUS) (Dafouz \& Smit, 2020). It is therefore used as a lens to zoom into the use of English as a medium of instruction within and across contexts (Bradford \& Brown, 2018a). In this sense, ROAD-MAPPING is appropriate for my study in framing a thorough understanding of EMI in a Vietnamese HE setting. This framework enables me to discuss the study's findings from a global perspective, and thus contribute to the literature on EMEMUS. In this section, I will first give an overview of the ROAD-MAPPING framework, and then discuss how it has been applied in recent research on EMI in different contexts.

\subsubsection{An overview of ROAD-MAPPING}

The ROAD-MAPPING framework was developed by Dafouz and Smit (2016) with the aim of building a conceptual framework to capture the dynamic nature of EMEMUS. The framework is designed to address a gap in purely linguistic models and conceptualise the complexity of multilingualism in $\mathrm{HE}$, as it "adopts a sociolinguistic perspective and views languages as the means to socialisation and the development of social practices" (Dafouz \& Smit, 2020, p. 43).

ROAD-MAPPING is conceptually underpinned by different theories including sociolinguistics, ecolinguistics and language policy research. While the focus of sociolinguistics is still on "the role of language in the construction of social relations and social organization" (Heller, 2008, p. 504), recently 
more importance has been attached to "the fluidity, complexity, and multi-functionality of English and other languages that academics, students, and administrators draw on for their institutionalized practices" (Dafouz \& Smit, 2020, p. 43). As such, sociolinguistics offers EMEMUS theoretical support to encapsulate discursive and other social practices. Ecolinguistics develops the ecology metaphor accentuating "explorations of the relationship of languages to each other and the society in which these languages exist" (Creese \& Martin, 2003, p. 161). In this way, multilingual universities can use an ecolinguistic perspective to build language diversity by maintaining the interrelatedness among the languages of their university, recognising their academic habitats, the agents themselves, and these "communicative practices and academic cultures in their global and local realizations" (Dafouz \& Smit, 2016, p. 401). This is in line with discussions on the three separable dimensions of language management, practices, and agents' beliefs that contribute to institutional language policies. Language policy in EMEMUS therefore takes account of current studies viewing discourse as a representation of social practices (Gee, 2014). In this view, discourse is considered to be the means by which members in construct social orders, organisations, and events in which they participate. In the framework of EMEMUS, discourse goes beyond the classroom where teaching and learning activities take place, and extends to wider aspects of education including "strategies for knowledge construction (both in the L1 and L2), issues regarding language pedagogy in multilingual university settings, or the language policies and practices stemming from these new teaching and learning situations" (Dafouz \& Smit, 2020, p. 45). Thus, discourse plays a significant role as an access point to the multifaceted nature of EMEMUS.

Starting from a foundation of sociolinguistic, eco-linguistic, and language policy considerations, the EMEMUS framework theorises six intersecting dimensions of discourse, namely: Roles of English (in relation to other languages) (RO), Academic Disciplines (AD), (language) Management (M), Agents (A), Practices and Processes (PP), and Internationalization and Glocalization (ING). These six dimensions are considered "as inherently complex, contextually bound, and intersecting dynamically with one another" (Dafouz \& Smit, 2016, p. 397). Roles of English addresses the position of English as a teaching medium in HE settings and encompasses a range of functions that English performs such as a gatekeeper or an outcome criterion. Academic Disciplines differentiates discourses operating in different disciplines, and the inherent epistemological characteristics of each academic discipline that can impact the teaching and learning practices in multilingual settings. Language Management refers to the language policy statements and 
declarations that have been adopted throughout the HE institutions. Agents are the stakeholders engaged in EMEMUS and can include individuals like teachers, students, and administrative staff, or collective institutions like departments, faculties, and student unions. Practices and Processes describe "the teaching and learning activities that construct and are constructed by specific EMEMUS realities" (Dafouz \& Smit, 2016, p. 407). Internationalisation and Glocalisation cover the international, global, national, and local forces and interests that influence universities to play multifaceted roles in society. In the framework, although all dimensions are likely to be equally relevant and independent, they interconnect with each other in the position of discourse as central point of access (see Figure 2). For example, (language) Management and Agents can be seen as contingent dimensions in that one requires the other. Managerial decisions are taken by higher educational agents, who, in turn, enact management. Similar dependencies can be identified between all other dimensions (Dafouz \& Smit, 2016, p. 411). This illustrates the dynamic and flexible nature of the ROAD-MAPPING framework which can be used in EMEMUS-focused research.

\section{Figure 2}

The ROAD-MAPPING framework for EMEMUS (Dafouz \& Smit, 2016, p. 404)

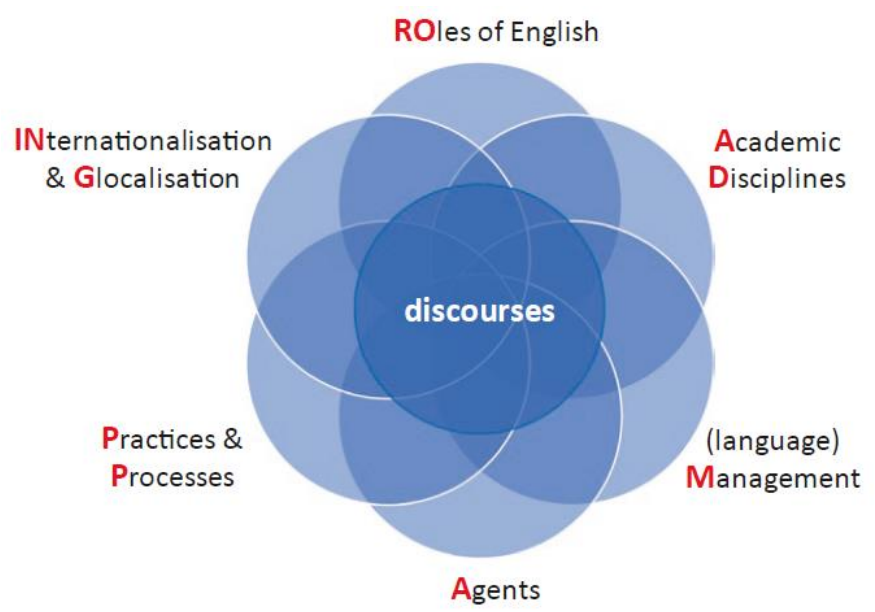

\subsubsection{Applications of ROAD-MAPPING in research}

The framework is designed "to function as a means to capture and analyse the dynamic, multi-layered and diverse nature of EMEMUS" (Dafouz \& Smit, 2020, p. 59). It is applied differently in a range of English-medium educational research contexts. More specifically, the framework can inform the phases of EMEMUS conceptualisation, support study design and methodology, and underpin data analysis and/ or discussion of findings. In their recent book, Dafouz and Smit (2020) 
provide examples illustrating how the ROAD-MAPPING framework has been used in academic studies.

One of the first applications of the framework investigates teacher beliefs about integrating content and language across four different HE institutions in Finland, the UK, Austria, and Spain (Dafouz et al., 2016). Researchers used ROAD-MAPPING as a conceptual framework to design the study and analyse the data. They also applied the framework in the process of developing codes from semi-structured interviews with teacher participants. Results from the analysis were combined with the dimensions of the framework focusing on Agents (A), Academic Disciplines (AD), Internationalisation and Glocalisation (ING), and Roles of English (RO). The authors reported using these dimensions to access the complexity of teachers' beliefs on the integration of Content and Language, which was "conceptualised diversely across sites and participants" (Dafouz \& Smit, 2016, p. 89).

Another application of the framework examined the roles and conceptualisations of English and other languages in three English medium multilingual universities in Thailand, Austria, and the UK (Baker \& Hüttner, 2017). The RO dimension of the framework was applied during the analysis of top-down codes. The results show that English and the other languages involved in these multilingual sites were given diverse roles and conceptualisations in that they were used as both a tool or target in education, or a lingual franca for inside and outside class communication (Baker \& Hüttner, 2017).

The ROAD-MAPPING framework has also been applied in research exploring English-medium education classroom discourse. For example, Komori-Glatz (2017) conducted a study to investigate how the peer-to-peer interaction conducted among students in teamwork worked to construct appropriate disciplinary language and achieve team goals. In this study, the framework was used not only as a conceptual framework but also as a meta-level methodological guideline for the discussion of the findings. The author mapped each of her findings using the framework dimensions to explain the respective findings. As one of the first large-scale empirical studies adopting Dafouz and Smit's (2016) ROAD-MAPPING framework, this research concluded that the framework "illuminates the multi-layered and multifaceted nature of the international university and reveals the tensions and synergies between various stakeholders and the resources and demands they bring to the Multilingual and Multicultural Learning Space" (Komori-Glatz, 2017, p. 290).

In an Asian context, recent studies have applied the ROAD-MAPPING framework in exploring the current state of EMI in different contexts. For example, Bradford and Brown (2018a) analysed 
data from their recent studies using the six dimensions of ROAD-MAPPING to identify and describe the realities of EMI in Japan. They found that EMI requires changes in pedagogy and classroom practices, but it is not yet fully utilised in HE. This presents an overall image of EMI as still developing in the Japanese context. Another example is Sameephet (2020) thesis which explores EMI practices in different language programmes in Thai universities. By applying ROAD-MAPPING to the findings, the researcher holistically looked at the language practices in and beyond classrooms in which the teachers and students tried to practise as much English as possible using linguistic resources available to them such as code-switching and translanguaging. The ROAD-MAPPING framework employed in Sameephet's study highlights the state of EMI in Thailand at a time when "the EMI policy was directed toward internationalisation, but its implementation can be seen as Glocalisation" (p. 235). These studies conducted using the framework have highlighted different characteristics of EMI practices and realities in different local contexts.

In Vietnam, there have been few studies applying the ROAD-MAPPING framework to the implementation of EMI in HE. The first study drawing on the framework was conducted by Dang and Moskovsky (2019). These researchers reviewed policy documents related to EMI in Vietnam's tertiary sector and provided an in-depth exploration of the multifaceted nature of EMI in Vietnamese contexts. Their findings suggested that the existing EMI policies still promote borrowing of EMI programes from overseas universities without "clear guidelines or recommendations for language management, internationalisation strategies, and proper involvement of multiple agents at different levels" (p. 1343). This study also uses the ROAD-MAPPING framework's six dimensions to identify the multi-dimensionality of EMI policies in Vietnam but has yet to examine EMI practices in Vietnamese HE institutions.

Researchers in EMEMUS increasingly apply the ROAD-MAPPING framework in their studies. This framework has the potential to conceptualise a comprehensive analysis of EME reality and informed action. It is also discursive, flexible, and dynamic enough to allow exploration of EME from different angles. The complex overlapping of the framework's dimensions suggests a further need for implementation in different contexts. In my study, the framework is employed to fill in this gap by investigating teachers' and students' experiences in EMI contexts in a Vietnamese university. Specifically, I harness six dimensions of the framework to discuss teaching and learning practices of both teachers and students in EMI courses in the Discussion chapter to illustrate the nature of EMI in a Vietnamese context. 


\subsection{Chapter summary}

This chapter reviews literature underpinning the teaching and learning of CLIL/EMI courses in $\mathrm{HE}$ in a digital age. The first part focuses on different aspects related to the integrating of content and language at tertiary levels where CLIL/EMI programmes have been increasingly implemented. The second part stresses the process of teaching and learning in connection with the development of digital technologies in HE. The third part describes the conceptual framework of ROAD-MAPPING and its application in research on the use of English as a medium of instruction in HE.

While CLIL/EMI has been a popular research focus in European countries, it has become a significant trend in Asian countries including Vietnam. However, there have been few studies reporting a comprehensive understanding of teachers and students' CLIL/EMI practices in Vietnamese HE contexts.

In terms of technology, a number of studies reviewed have reported on the various effects of digital technologies in academic settings, as well as exploring influences on teachers and students' use of educational technologies. While there have been quite a few studies investigating the impacts of digital technologies on language teaching and learning generally, little evidence has been reported regarding the use and impacts of technologies in CLIL/EMI contexts.

My study recognises these gaps in its aims to investigate the experiences of teachers and students in EMI courses at one Vietnamese HE institutions. To gain deeper understandings of the multifaceted nature of EMI practices, this study uses the lens of the ROAD-MAPPING framework as discussed above. The following chapter outlines the study's methodological approach. 


\section{Chapter 3. Methodology}

This chapter builds on the theoretical interpretation and literature reviewed in Chapter 2. In particular, the emphasis on the multifaceted nature of English-medium Instruction in a digital age has directed my methodological decisions in research design. This chapter starts with justifying the selection of the qualitative multiple case study underpinned by an interpretive paradigm, then provides an overview of the research setting and the process of purposefully selecting participants. The chapter finally details the procedures of data collection and data analysis and specifies varying strategies to maintain the rigour of the research.

\subsection{Research question}

In this study, I examined how teachers and students perceived and adapted to the emerging context of EMI. I also investigated the ways that digital technologies were used for learning and teaching. The overarching research question was: How are digital technologies used in an EMI context in Vietnamese higher education?

The following sub-questions support this main research question:

1. How do teachers and students use digital technologies in an EMI environment?

2. How do teachers perceive their students' learning to have developed through digital technologies in an EMI environment?

3. How do students perceive their learning to have developed through digital technologies in an EMI environment?

To answer these questions, I designed my research based on the procedure described by Creswell (2018a) (see Figure 3). Following this procedure, I aimed to select cases that were likely to show different perspectives on the topic. In each case, I collected data from three sources of information: teacher interviews, classroom observations, and student focus-group discussions. These data were processed thoroughly to prepare for the next step of analysis. I analysed the data by identifying codes in the transcripts and field-notes, then describing each single case, and finally developing themes from cross-case analysis. During this step, I not only addressed the research questions but also linked to significant themes or arguments identified in the literature review. This helped me draw a valid conclusion from the findings. 
Figure 3

The research design

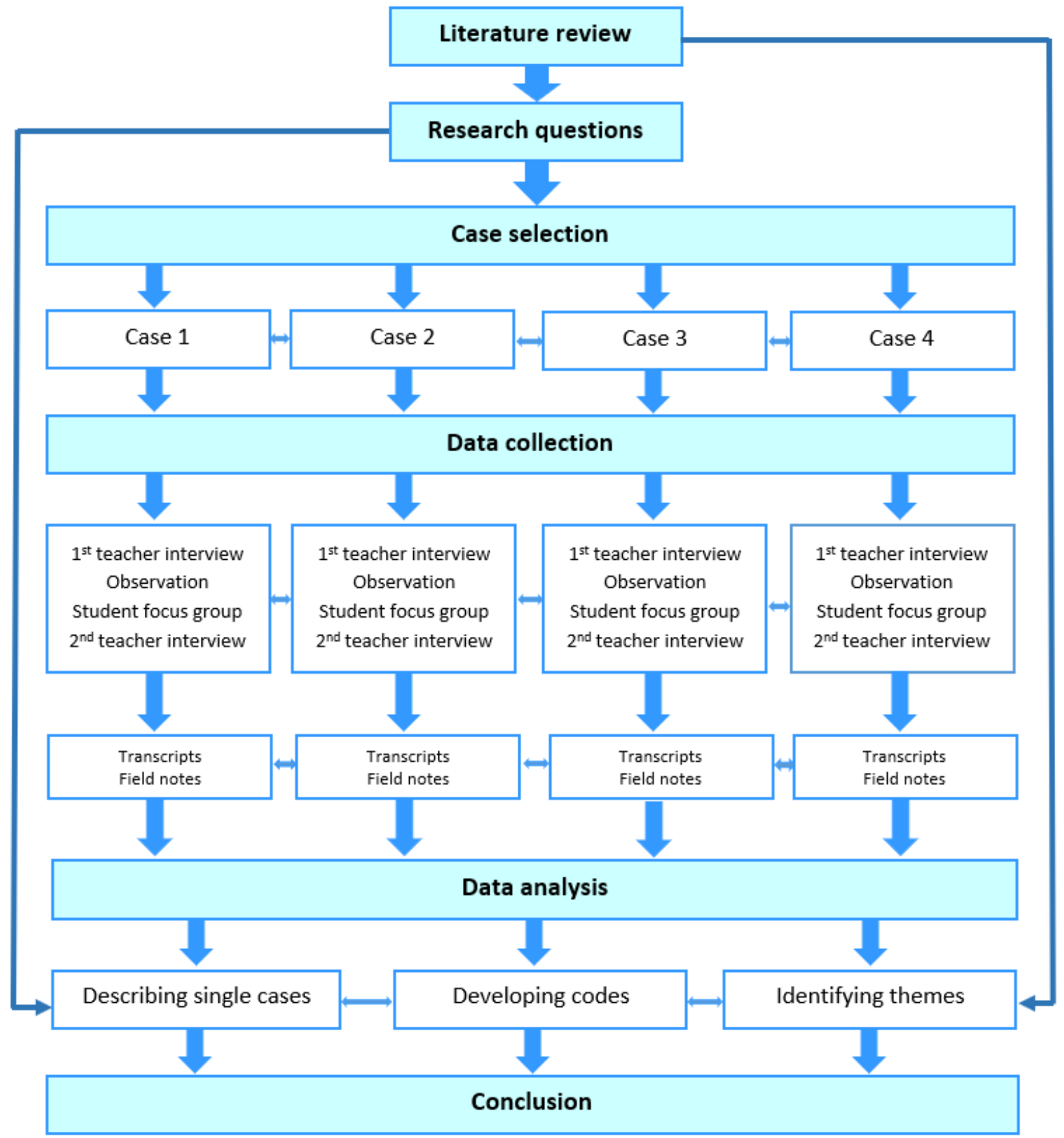




\subsection{Interpretive qualitative research}

Qualitative research methodology was used to conduct the study. Qualitative research is defined as "an approach that allows researchers to examine people's experience in detail, by using a specific set of research methods" (Hennink et al., 2020, p. 9). As a qualitative approach looks at a social or human problem from the perspective of participants (Creswell, 2018b), its characteristics are appropriate for understanding the perceptions of both teachers and learners when they had to adapt to a new learning and teaching context. Observations helped me understand their teaching and learning practices (with a focus on digital technologies) in a real context. This aligns with the aims of qualitative research to explore how people experience and behave in their natural settings (Hennink et al., 2020).

My research was underpinned by an interpretive paradigm which "locates the observers in the world" by using interpretation and observation to understand the social world (Denzin \& Lincoln, 2013). This means that how people interpret the phenomenon they observe reflects their own perceptions which have developed within a social, cultural and personal context. As such, an interpretive qualitative approach most effectively supported an investigation of the everyday and exceptional experiences of both teachers and students related to digital teaching and learning in their naturalistic setting of CLIL/EMI.

Reflexivity plays an important role in qualitative research. Reflexivity refers to "the awareness that the researcher's values, background, and previous experience with the phenomenon can affect the research process" (Cope, 2014, p. 90). It helps to maintain researchers' engagement in "critical self-awareness throughout the research process" (Probst, 2015, p. 46). Reflexive researchers reflect on the influence of their own values, preconceptions, behaviours or presence and those of the participants when interpreting data (Parahoo, 2014). As an insider (Denzin \& Lincoln, 2018) who had worked as an English teacher at the university, I was aware that this could influence how the participants responded to me, and how I interpreted and analysed the data, so I was sensitive to these issues throughout the study. I used reflexivity to identify similarities and differences in the participants' beliefs and practices to my own by talking to and observing participants. Specifically, when interviewing the participants and transcribing the data, I always debriefed with them to ensure my understanding of their viewpoints in interpreting the data and sent them transcripts for checking. Additionally, when analysing the data, I kept returning to the raw data, the context of data collection, and the literature. These practices helped to make sure that I captured the participants' perspectives 
rather than my own and enabled me to "situate findings in existing knowledge" as well (Probst, 2015, p. 44).

\subsection{Multiple-case study}

Case study is a qualitative research methodology. It is "the study of the particularity and complexity of a single case, coming to understand its activity within important circumstances" (Stake, 2013, p. xi). Yin (2018) recommends using case study when "a 'how' or 'why' question is being asked about a contemporary set of events, over which the investigator has little or no control" (p. 13). Using case study allows researchers to explore a phenomenon as it is influenced by the context in which it is situated (Denzin \& Lincoln, 2018). Case study allowed me to investigate participants' experiences and practices through classroom observations, interviews and focus group interviews.

Multiple case study is the study of a number of cases to "investigate a phenomenon, population or general condition" (Stake, 2005, p. 445). In multiple-case study, researchers examine several cases in order to show different perspectives on the issue (Creswell et al., 2007). This allows researchers to explore both the similarities and the differences within and between cases. Each single case is described as "a phenomenon of some sort occurring in a bounded context" (Miles, 2014). This implies that a case must be studied within certain boundaries. The boundaries can be time, location or "the number of people who could be interviewed or observations that could be conducted" (Merriam, 2010, p. 456).

My study focused on multiple cases at one university in Vietnam in the specific context of undergraduate economics-related courses taught in English by subject teachers. There is no specified ideal number of cases in a multiple case study, yet four to 10 cases are likely to be enough to maintain the interactivity as well as the benefits of multiple case study (Stake, 2005). The study of each information-rich case can provide significant insights and lead to in-depth understanding (Patton, 2015) as "the real business of case study is particularization, not generalization" (Stake, 1995, p. 8). I investigated four individual classes, each of which had a different major. Each case involved one subject teacher and a class of 40 to 50 students working in an EMI course. The subject teachers and students came from four different disciplines including Management, Economics, Finance, and Electronic Commerce. While one class was in the first year, three others were in the third year. Data collected were first analysed within each case before an analysis was conducted across cases to find common patterns which were then labelled, refined and adjusted as a final result (Merriam, 2010). 


\subsection{Research setting}

This study was conducted in one university situated in Vietnam that offers undergraduate and postgraduate programmes to thousands of students who major in the field of economics including economics, commerce, business management, finance, banking, accounting, and marketing. This university is recognised as one of several public autonomous institutions in Vietnam which runs EMI programmes for mainstreamed students rather than targeting select groups of students in international programmes.

In early 2016, this university began an Advanced Programme for undergraduate students alongside the High-Quality Programme that started in 2011. As part of the implementation, the university offered training courses in EMI designed by Cambridge University for teachers involved in the programmes and who would start teaching their subject in English in 2016. Teachers in the EMI courses participated in 24 hours of classroom training and 40 hours of online learning. There were eight modules in each course focusing on using English to teach in various contexts including lectures, seminars, small groups and practical sessions, and tutorials and supervision. Seventy-four teachers had been awarded an EMI certificate by 2018.

At the time of my data collection in 2017, major modules of economics were taught in English by subject teachers. Each module lasted from 11 to 15 weeks and students had one three-hour lecture a week. Along with subject modules, all students had six modules of English in Business taught by teachers of English. In these modules, students were offered lessons in integrated skills of communicative business English based on the main course books including Market Leader Business English (Cotton, Falvey, \& Kent, 2006) and the Supplementary Materials (entitled Practice Books 1, 2, 3, and 4) compiled by the English teachers. Students were supported with an e-learning system in every subject and an EDO (English Discoveries Online) account issued by a testing and educational organisation in Vietnam so that students could improve their English skills independently online. The EDO system was used alongside six modules of English that students in the High-Quality class had to take. With the aim of providing students with extra practice in general business English, the EDO system is a learning webpage providing students with different sections such as Support to contact teachers, My Course to study at home, and Community to interact with others.

The university invested in developing technological facilities and provided teachers and students with opportunities to use technologies in their teaching and learning. In mid-2016, the university piloted a system of e-learning using a Learning Management System (LMS) to encourage 
teachers to incorporate digital technologies such as power point, Moodle, and web-based forums into their teaching. At the time of my study, all teachers were trained to integrate the LMS in their courses. The teachers were encouraged to conduct at least one lecture via the LMS during their courses and all classrooms were equipped with one desktop computer, one data projector with screen, and a sound system. The system of e-library and wireless Internet access became available and accessible to all teachers and students at all campuses.

When I commenced my study in late 2016, both EMI programmes and the LMS appeared to be new to teachers and students at the university. This made it an appropriate context for me to study the teachers and students' experiences of teaching and learning EMI courses with the assistance of digital technologies.

\subsection{Selection of participants}

Purposeful sampling was used to recruit participants for the research. Purposeful sampling is a set of strategies which researchers can use to intentionally choose participants or sites to best "understand the problem or the research question" (Creswell, 2018b, p. 189) . In this study, my purpose was to select teachers and students who were participating in EMI programmes and came from different disciplines. Purposeful sampling is the most suitable approach for selecting teachers and students from different schools and majors to include diverse perceptions and experiences in using digital technologies in a CLIL/EMI context. I had expected to recruit teachers who had completed the EMI training and received EMI certificates, but not all of the EMI teachers had completed this training. This meant that I recruited one participant who had not untaken EMI professional development.

I started the process of selecting participants by seeking permission from the university authority. I contacted the principal of the university and the heads of schools for permission to conduct research in their schools. Both the principal and the heads of schools were given information sheets stating the objectives of my study (see the sample in Appendix A). They agreed to sign consent forms for the study (see the sample in Appendix B).

The principal allowed me to access a list of teachers involved in the High-Quality and the Advanced Programmes. I emailed 16 teachers who were scheduled to teach a course in August 2017 and provided them with information detailing my research and their possible contributions (see Appendix A). Three teachers agreed to join my study. After emailing five other teachers scheduled to teach a course in October 2017, one more teacher agreed to participate. I arranged personal 
meetings with the four volunteers individually to discuss consent and arrange schedules for interviews and observations (see Appendix B). Although I had had a working relationship with those teachers as colleagues, I had not had much professional contact with them prior to the study as we worked in different schools. Moreover, I did not have a management role at the university, so I did not possess any power or authority, which may have affected their choice to participate.

The four teacher participants, two males and two females aged from 32 to 49, were subject teachers responsible for the teaching of different economics subjects in four different schools at the university (see Table 1). All had many years' experience in teaching their subjects in Vietnamese. However, three of them were new to the context of teaching through English while the fourth was experienced in using English to teach her subject. One teacher was on the waiting list for the next EMI training course. These teachers' highest qualification was a master's degree and three of them were pursuing their doctorate study. I used pseudonyms for the teacher participants to protect their identity.

Table 1

A summary of teacher participants' information

\begin{tabular}{|l|c|c|c|c|}
\hline & Vincent & Nathan & Taylor & Nancy \\
\hline Gender & Male & Male & Female & Female \\
\hline $\begin{array}{l}\text { Years of teaching experience } \\
\text { (in Vietnamese) }\end{array}$ & 5 & 5 & 11 & 7 \\
\hline $\begin{array}{l}\text { Years of teaching experience } \\
\text { (in English) }\end{array}$ & 0 & 1 & 7 & 1 \\
\hline EMI certificate & No & Yes & Yes & Yes \\
\hline Teaching courses & Management & Economics & Finance & Electronic commerce \\
\hline
\end{tabular}

At the initial meeting, I provided participants with detailed information about the research, their roles in the research, and their right to withdraw from the study at any time. The teachers were asked if they would be available for two interviews and classroom observations and each agreed to participate. The teachers expressed their concerns about being observed as they were not confident in using English. I reassured them my study was not about evaluating the effectiveness of their teaching practices or use of English but instead I wanted to explore their use of digital technologies and learn about their experiences of teaching EMI courses. After this discussion, the teachers gave consent for audio-recording of interviews and/or video-recording of classroom observations. All four teachers agreed to audio-recording, and three to video-recording. 
The teachers selected one of their classes as a focus for the study. Each teacher arranged time for me to observe one class lecture. I recruited students for focus groups from the suggested classes. After the classroom observations, I spent 15 minutes talking to students about my research. I provided each student with information about the study and a form on which students were invited to share personal information including their names, ages, genders, contact information, their levels of confidence in learning subject content, literacy about digital technologies, English competence, and information about providing consent to participate in a focus group interview (see Appendix D). Students interested in focus group discussions were able to specify their available time on the sheets.

With 71 students out of 169 expressing interest in joining a focus group discussion, I applied criteria to select potential students in each class. I considered their gender and fields of confidence to maintain the diversity of the focus groups. I also took the students' availability into account to make their participation easier. I contacted ten potential students in each class via phone and email and 26 students, including nine males and 17 females, agreed to participate in focus group interviews. However, two students in Taylor's class did not turn up at the discussion due to personal reasons, so the final number of students for focus groups was 24 (see Table 2).

\section{Table 2}

A summary of students participating in focus group discussions from each class

\begin{tabular}{|l|c|c|c|c|c|}
\hline & Vincent & Nathan & Taylor & Nancy & Total \\
\hline Students in class & 43 & 40 & 45 & 41 & 169 \\
\hline Students in focus group & 7 & 6 & 4 & 7 & 24 \\
\hline
\end{tabular}

These students were aged from 18 to 20 and majored in different areas of economics. Six of the students were in their first year, and 18 were in their third year. They signed a consent form at the beginning of the discussions (see Appendix B), and I emailed students who were not selected for the interviews to thank them for their involvement and interest in the study.

\subsection{Data collection}

Data were collected during the second semester of the school year, from August to December. Three major sources of data included two teacher interviews, one classroom observation and one student focus group in each case. The data collected were transcribed in Vietnamese and entered into N-Vivo11. Transcripts were used for data analysis and significant parts were translated into English for findings reports. My colleagues, who were doing their Ph.D. in Applied Linguistics, helped me check my translation and back translate all essential parts. The use of the back-translation 
technique, which is translating from target language into source language (Chen \& Boore, 2010), helped to make sense of the scripts in both languages. Participants selected the best time for their involvement (see Table 3).

\section{Table 3}

Dates of contacting participants and collecting data

\begin{tabular}{|l|c|c|c|c|}
\hline & Vincent & Nathan & Taylor & Nancy \\
\hline First meeting & $24 / 07 / 2017$ & $31 / 07 / 2017$ & $05 / 09 / 2017$ & $30 / 10 / 2017$ \\
\hline First teacher interview & $01 / 08 / 2017$ & $14 / 09 / 2017$ & $19 / 09 / 2017$ & $11 / 11 / 2017$ \\
\hline Classroom observation & $07 / 08 / 2017$ & $21 / 09 / 2017$ & $26 / 09 / 2017$ & $18 / 11 / 2017$ \\
\hline Student focus group & $11 / 08 / 2017$ & $27 / 09 / 2017$ & $05 / 10 / 2017$ & $23 / 11 / 2017$ \\
\hline Second teacher interview & $14 / 08 / 2017$ & $03 / 10 / 2017$ & $18 / 10 / 2017$ & $15 / 12 / 2017$ \\
\hline
\end{tabular}

I had piloted the data collections tools prior to commencing the data collection process. Specifically, I interviewed two colleagues who used to be subject teachers and were doing their Ph.D. at Victoria University of Wellington. My supervisor allowed me to observe one of her lectures and conduct a stimulated interview with her after class. This helped me improve my question prompts and skills for collecting data with participants.

\subsubsection{Teacher interviews}

Interviews are an important source of information which allows researchers to identify details that they cannot get from participants by observing them (Creswell, 2018b). Interviews can be conceptualised as guided conversations between researchers and people participating in the research that explore the topic from the perspectives of the participants. In an interview, interviewees not only provide interviewers with their insights but also offer "other relevant sources of evidence" such as thoughts, feelings or experiences (Yin, 2013, p. 108). Semi-structured teacher interviews were used in this study.

Semi-structured interviews are one of the most frequently-used methods for data collection in qualitative research (Kajornboon, 2005). They are interviews in which interviewers use specific questions which convey their foreseen information and open-ended questions "to elicit unexpected types of information" (Hove \& Anda, 2005, p. 2). The interviewers control the conversations to some extent using open questions and flexibly explain or ask for clarification to prompt the respondents as required (Corbetta, 2003). In such interviews, interviewees are directed to some degree but allowed to express their opinions about a topic as thoroughly as possible (Longhurst, 2009). As stated in the 
rationale for choosing semi-structured interviews by Horton et al. (2004), the interviewees are provided with "a degree of freedom to explain their thoughts and highlight areas of particular interest and expertise that they felt they had" (p. 340).

After the initial meetings, I conducted the first interviews beginning with the teachers signing consent forms. The semi-structured interviews were guided by questions and prompts (see Appendix E). Being able to prompt and probe deeper into the topic enabled me to "ascertain participants' perspectives regarding their experience pertaining to the research topic" (Mclntosh \& Morse, 2015, p. 1). I used Vietnamese (their preferred language) with teacher participants and audio-recoded the conversations. Each interview lasted from 30 to 45 minutes. I listened to the recording of each interview again before conducting interviews with the next teachers. This helped me revise prompts, improve ways of asking questions and modify the focus of the interviews.

I conducted the second interviews with teachers after finishing the classroom observations and student focus groups. Three of these interviews were video simulated recall interviews in which the teachers were shown clips from videos of their own teaching and then invited to expand on their decision-making (Schmid, 2011). This strategy offered me an opportunity to probe teachers' perspectives on their teaching practice (Tripp \& Rich, 2012). I tried to schedule these interviews soon after the observations. However, this was not possible. Two teachers were away on one-week business trips while the two others were busy with their PhD study and teaching schedules. This meant that the second teacher interviews took place about two weeks after classroom observations. Before the interviews, I watched the video-recordings of the observed lectures. I noted down any points that needed exploring. In the interviews, I paused the clips in particular places and prompted teachers to talk about their teaching practice. In the interview with the teacher who did not wish to be videoed, I used my observation field-notes to stimulate her answers. I focused these interviews on their experiences of teaching EMI courses and their use of digital technologies in class.

I sent the teachers transcripts of the audio-recorded interviews for member checking to maintain the accuracy of data collected (Stake, 2005). Three teachers accepted the transcripts, and the other added a point related to her English competence.

\subsubsection{Observations}

Observations are defined by Creswell (2018b) as a process in which researchers observe people and places at a research site in order to collect "open-ended, first-hand information" (p. 190). 
For a case study, the observation is a source of evidence capturing particular occurrences at a certain period of time in a particular setting (Yin, 2013).

One week after the first interviews, I visited the classes to observe a lecture. Each lecture lasted three hours. I carefully observed how both teachers and students responded to the EMI setting and how they utilised digital technologies, and video-recorded three of the classes to allow for stimulated recall interviews. Such observational evidence supported the understanding of the participants' actual use of the technology and any problems taking place in classroom (Yin, 2013). During the observation, I recorded the following information:

- The kinds of interactions between teachers and students.

- Teachers' activities including how they communicated subject content to students, gave instructions in English, and used digital technologies in teaching.

- Students' activities including their engagement with subject content and English use, participation in discussion or group work, and how they used digital technologies in class.

In addition, I made notes on the interrelatedness of subject content, the use of English and digital technologies. The guiding framework for observation is illustrated in Figure 4.

\section{Figure 4}

Observation chart

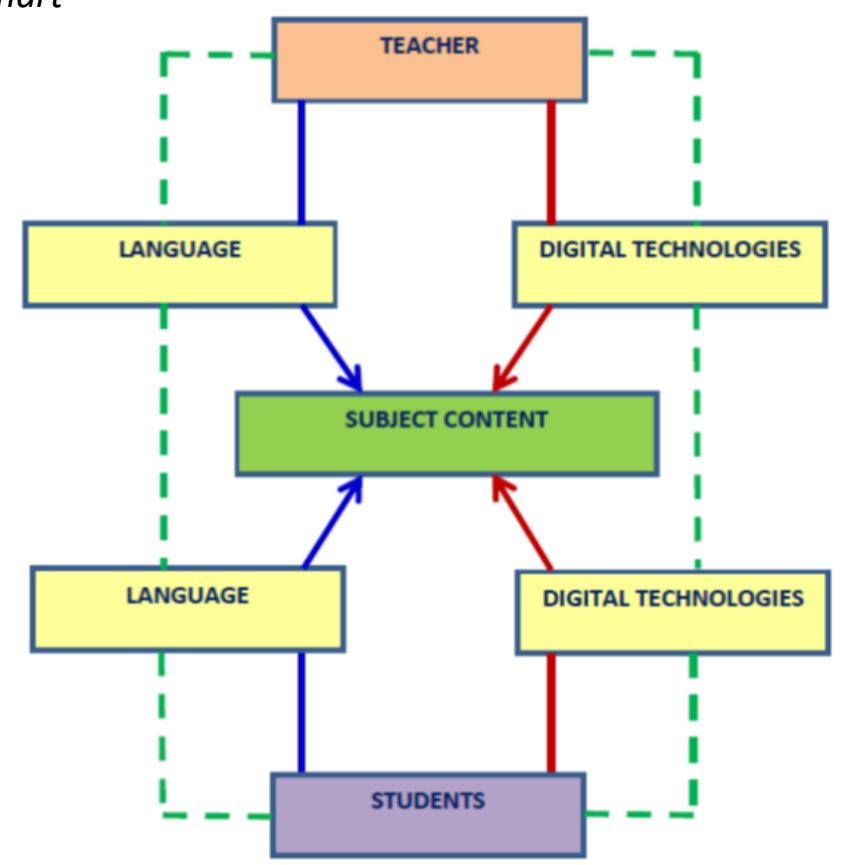

- Teacher - Students interactions

Teacher's and students' use of English to deal with subject content

Teacher's and students' use of digital technologies to deal with subject content 
I used an observational checklist and took field-notes during the observation so that I could review after each class visit to prepare for the next cases. The checklist and field-notes (see Appendix C) were adapted from the model of Sheltered Instruction Observation Protocol (SIOP, 2008) and digital age learning matrix (Starkey, 2011).

\subsubsection{Student focus group interviews}

Focus group interviews have been extensively used in qualitative research in social sciences including education over the past two decades (Harskamp \& House, 2019). They are described as a technique to collect information on a specific topic from the discussions of a group of participants who gather in one place during a specific time (Daniela Dal Forno et al., 2017) and allow for "an indepth understanding of social issues" (Nyumba et al., 2018, p. 20). For this study, focus group interviews were applied to "capitalize on group dynamics to stimulate discussion" (Guest et al., 2017, p. 693) which was likely to generate a wide range of opinions and surface the participants' beliefs (Krueger, 2014), and to encourage interactions between researcher and participants and among participants themselves to potentially elicit substantive information about the topic (Rosenthal, 2016).

I scheduled student focus groups within the week of the classroom observations at a time and venue that suited the students. Interview questions were sent to students via email three days prior to the focus group discussions. At the beginning of each discussion, after we discussed their rights and responsibilities in the research, students gave written consent. Students agreed on certain ground rules for their focus group interview (see Appendix D). All focus group discussions were conducted in Vietnamese at the students' request. I used the question prompts to frame a discussion of their experiences learning in the new EMI context that required them to use digital technology while learning content knowledge through English. I audio-recorded the discussion so that I could transcribe the recording and prepare for the next groups. Discussions lasted 45 minutes to 60 minutes. I summarised the ideas from each focus group and sent a summary to the students for modifications and checking. All students approved the summaries.

\subsection{Data analysis}

Data analysis is an essential stage in the study where researchers "make sense out of text and image data" (Creswell, 2018b, p. 195). Data from teacher interviews, observation field notes and students' focus group discussions were managed both manually and using computer software so that I could access any piece of data at any time (Merriam, 2014). Transcripts and field-notes of four cases 
were separated into four different document folders with identified labels. I created a data project in Nvivo 11 to manage and process all data. The process of data analysis included individual case descriptions and then a thematic cross case study analysis. Within-case analysis identified distinctive contextual features, and cross-case analysis enabled the building of "abstractions across cases" (Merriam, 2014, p. 234).

An abductive approach was applied for analysing data. Abductive analysis is defined as an approach "aimed at generating creative and novel theoretical insights", thus data were analysed not only based on emerging themes, but also on themes established in the literature (Timmermans \& Tavory, 2012, p. 180). The process of thematic analysis was employed to systematically identify, organise, and offer insight into patterns of meaning (themes) across the data (Clarke \& Braun, 2017). The development of themes was conducted using the method suggested by Vaismoradi et al. (2016). This is a "stage like and reiterative method" which includes four phases: initialization, construction, rectification and finalization (see Figure 5) (p. 103). In this process, I coded and categorised each case and created themes across cases.

\section{Figure 5}

Phases and stages of theme development adapted from Vaismoradi et al. (2016)

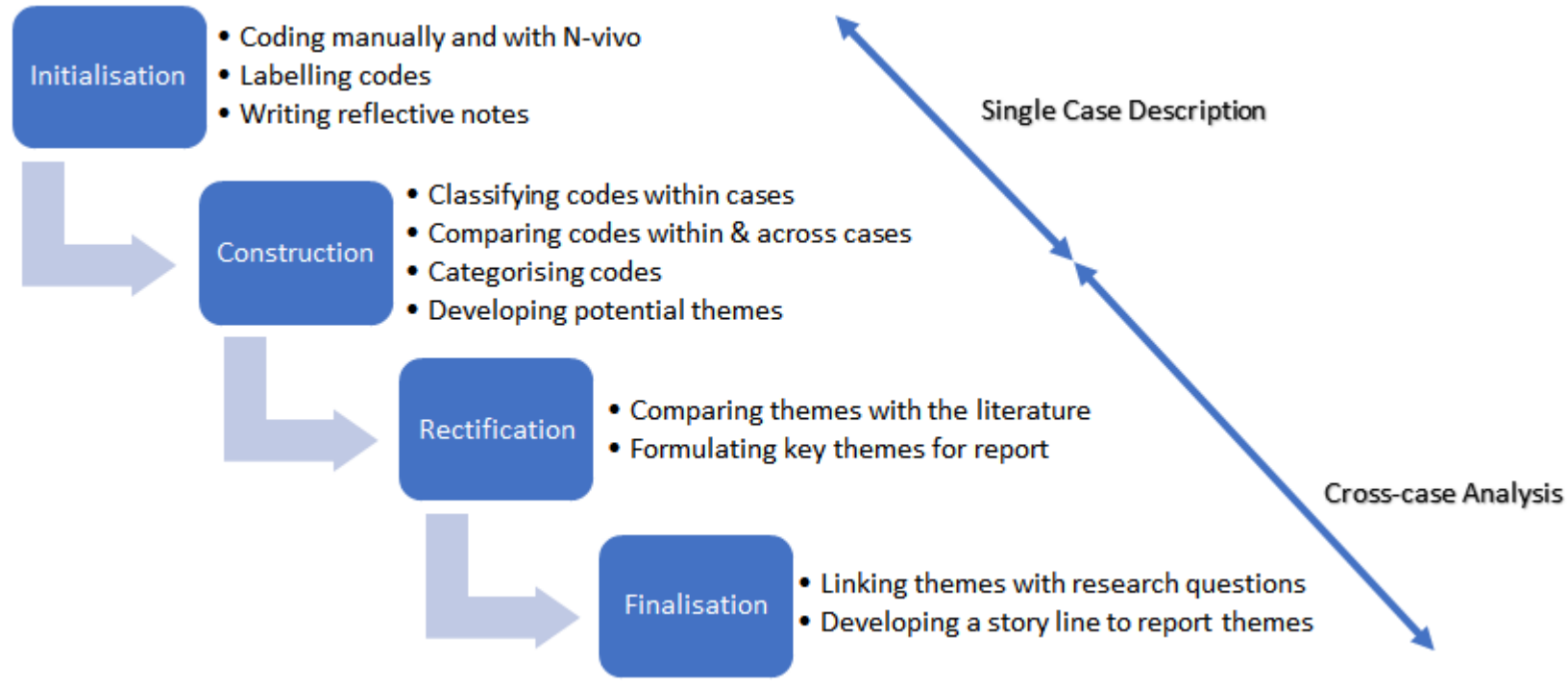

\subsubsection{Individual case study analysis}

Individual case study analysis aimed "to describe, understand, and explain what has happened in a single, bounded context" (Miles et al., 2013, p. 100). I started the first phase of initialisation by reading the transcripts and notes through carefully to get a general sense of the 
information and reflect on its overall meaning (Creswell, 2018b). This helped me become familiar with the data and prepare for coding.

Coding the data is a vital step in thematic data analysis and involves categorising segments of data represented by a particular phrase (Rossman \& Rallis, 2011). Codes were developed not only deductively from the literature review findings but also inductively from the data themselves (Miles et al., 2013). I reread transcripts and field-notes for each case several times and used both descriptive and in vivo strategies to code the data. I used descriptive coding to select a word or short phrase summarising a chunk of data and this phrase was then assigned to similar data chunks in the transcripts and field-notes. Using in vivo coding, I created codes using "words or phrases from the participants' own language in the data" (Miles, 2013, p.72). These words or phrases were translated into English to report the results, and the back-translation technique was also used to make sense of the codes.

I conducted the coding both manually and with N-Vivo11. I commenced the process by highlighting and labelling each data chunk with codes written on sticky-notes in different colours. This strategy of coding was repeated for each study case. Concurrently, I coded transcripts and field-notes saved in the N-Vivo 11 project. This code-recode strategy, which refers to the procedure in which the same data were coded twice in a period of time (Krefting, 1991), helped me compare the results of the coding process. Moreover, as N-Vivo made it easier to compare and contrast different codes (Creswell, 2018b), coding both manually and in N-Vivo allowed me to review and revise each code as new codes emerged. This also helped me compile the list of codes and look for reoccurring patterns effectively. During the coding process, I wrote reflective notes about features of the data and continued to reflect on my understanding and interpretation of codes or segments of transcripts, which helped me "remember, question, and make meaning of data" (Vaismoradi et al., 2016, p. 103).

Having completed the initial coding, I started the second phase of construction with the classification of codes within each case. This aimed to prepare for a full description of an individual case. Based on the participants' accounts, I grouped the relevant codes into six different components: the context, the teacher, the teaching of content knowledge, the use of English, the use of digital technologies, and the students.

These six categories allowed for a full description of each case. The context was described to clarify characteristics of the school and programmes, ensuring the boundary of each case and unveiling any latent factors that might have influenced participants. The teacher category provided 
information related to the teachers' academic background, teaching experience and philosophy that enabled them to make decisions in the new context. The teaching of content knowledge referred to the teachers' pedagogical approach in teaching subject matters to students. This section looked at the teachers' confidence in content knowledge and the reasons for their choice of teaching activities and perceptions. The use of English showed how teachers used English for interaction and instructions in class. This part explored the teachers' thoughts and actions taken in experiencing the teaching through English. The use of digital technologies investigated how the teachers used various technological tools and resources to improve their teaching practice and enhance students' learning. Finally, the students was the section describing the students' use of English and digital technologies in learning, and included students' perceptions of the new learning context and how they dealt with that situation.

Evidence was included in each case description, in the form of significant quotes from participants translated from Vietnamese into English (again with back translation procedures in place). I applied the digital age learning matrix developed by Starkey (2011) to examine the activities in which teachers and students used digital technologies for their teaching and learning. This tool allowed me to evaluate classroom activities that incorporated digital technology. The matrix explored how categories of digital technologies worked with aspects of learning (see Table 4).

\section{Table 4}

The digital age learning matrix (Starkey, 2011)

\begin{tabular}{|l|l|l|l|l|l|l|}
\hline $\begin{array}{l}\text { Aspects of } \\
\text { learning: } \\
\text { Digital } \\
\text { technology use: }\end{array}$ & Doing & $\begin{array}{l}\text { Thinking } \\
\text { about } \\
\text { connections }\end{array}$ & $\begin{array}{l}\text { Thinking } \\
\text { about } \\
\text { concepts }\end{array}$ & $\begin{array}{l}\text { Critiquing } \\
\text { and } \\
\text { evaluating }\end{array}$ & $\begin{array}{l}\text { Creating } \\
\text { knowledge }\end{array}$ & $\begin{array}{l}\text { Sharing } \\
\text { knowledge }\end{array}$ \\
\hline $\begin{array}{l}\text { Accessing } \\
\text { information }\end{array}$ & & & & & & \\
\hline $\begin{array}{l}\text { Presenting } \\
\text { information }\end{array}$ & & & & & & \\
\hline $\begin{array}{l}\text { Processing or } \\
\text { creating digital } \\
\text { objects }\end{array}$ & & & & & & \\
\hline $\begin{array}{l}\text { Gaming or } \\
\text { interactive } \\
\text { programmes }\end{array}$ & & & & & & \\
\hline $\begin{array}{l}\text { Communicating } \\
\text { or collaborating }\end{array}$ & & & & & & \\
\hline
\end{tabular}


In terms of English use, I used the framework of principles established by Gleeson (2015) to investigate the content-based language teaching and learning in each case (see Table 5). Specifically, I looked at the teachers' teaching practices with reference to each principle to reflect on connections between teaching activities and language learning and teaching.

\section{Table 5}

The framework of principles in content-based language teaching and learning (Gleeson, 2015)

\begin{tabular}{|l|l|l|l|l|l|}
\hline Principles & $\begin{array}{l}\text { Knowing } \\
\text { how English } \\
\text { is learnt } \\
\text { through } \\
\text { content }\end{array}$ & $\begin{array}{l}\text { Creating } \\
\text { connections } \\
\text { between the } \\
\text { students' } \\
\text { experiential } \\
\text { background } \\
\text { and new ideas }\end{array}$ & $\begin{array}{l}\text { Sustaining } \\
\text { academic rigour } \\
\text { and maintaining } \\
\text { high } \\
\text { expectations }\end{array}$ & $\begin{array}{l}\text { Knowing the } \\
\text { academic } \\
\text { language } \\
\text { demands of } \\
\text { the subjects } \\
\text { and } \\
\text { sustaining a } \\
\text { focus on } \\
\text { academic } \\
\text { language }\end{array}$ & $\begin{array}{l}\text { Engaging } \\
\text { students in } \\
\text { quality } \\
\text { interactions that } \\
\text { promote } \\
\text { productive and } \\
\text { receptive skills }\end{array}$ \\
\hline
\end{tabular}

\subsubsection{Cross case study analysis}

Cross case analysis was conducted to deeply understand and clearly explain multiple cases (Miles et al., 2013, p. 101). This helped to identify both similarities and differences across cases and highlight the unique qualities of each case by emphasising the impact of a particular context or situation on the phenomenon (Stake, 2005).

The second phase continued with the construction of themes. Figure 6 illustrates how a potential theme was constructed. I started with clustering codes sharing certain similarities into larger potential groups. These groups conveyed each "unifying idea that characterizes the experiences of participants" (Vaismoradi et al., 2016, p. 205). Then I compared codes to find out whether the same codes occurred across cases. The related codes were grouped into categories that explicitly described the meaning of the code patterns. These categories were labelled and scrutinised to provide details for theme development. As themes are defined as implicit topics organising a group of repeating ideas (Ryan \& Bernard, 2003), the categories covering frequently repeated codes were considered in the abstraction process as potential themes. All potential themes were described and defined clearly to be reconsidered in the next step. 


\section{Figure 6}

An example of the construction of potential themes

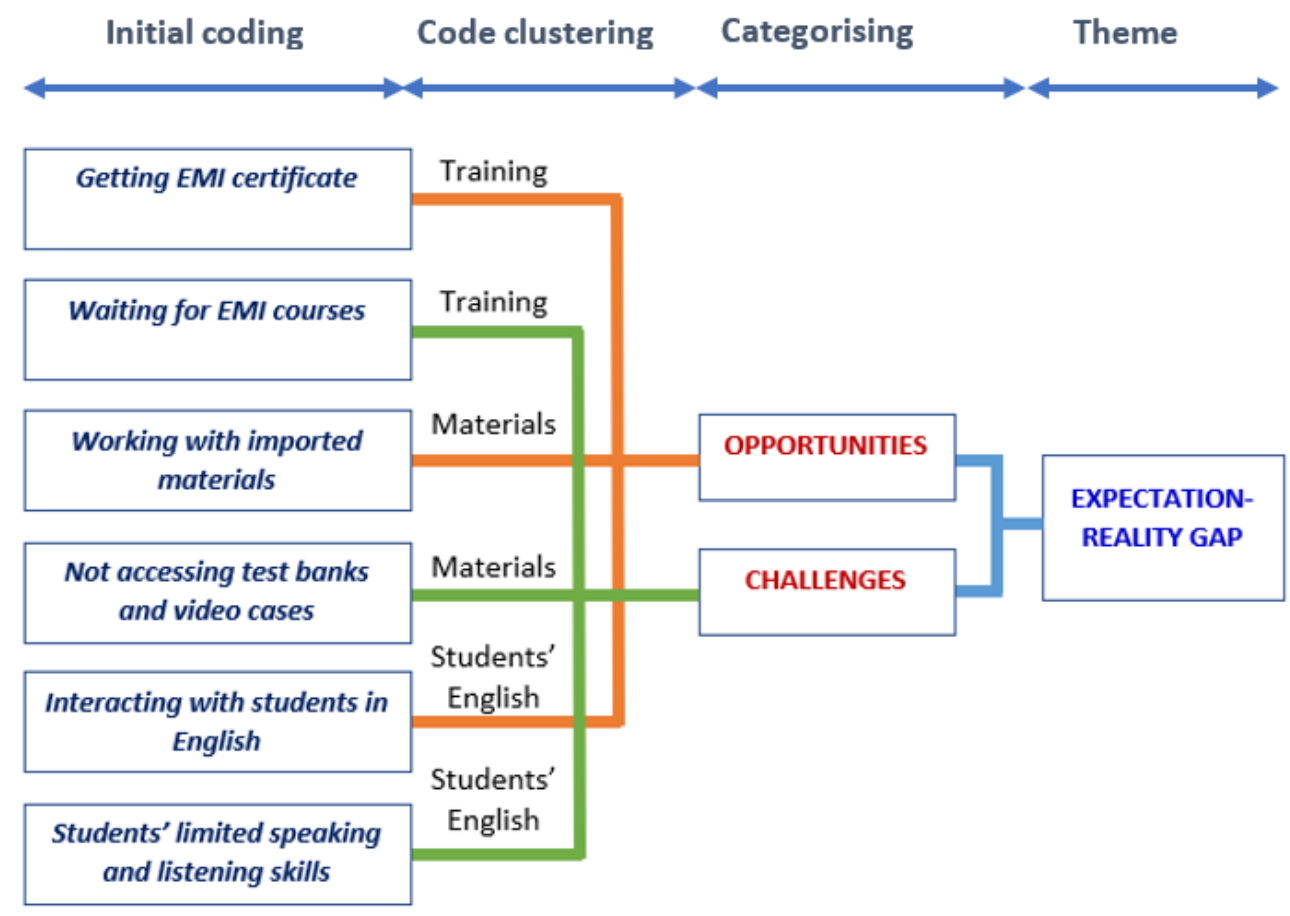

In the rectification phase, I returned to the literature review, comparing all the potential themes with themes suggested in my review. This helped to decide appropriate theme names, and I then described these themes to ensure they had analytical value in the final study reports. Finally, I drew upon the literature, the research questions and the themes to report the findings.

\subsection{Reporting findings}

Findings are the outcomes of the study reporting what researchers "learned or came to understand about the phenomenon" (Merriam, 2014, p. 277). In this study, the findings were reported with supporting evidence from the within-case and cross-case analysis of the data collected from the participants' interviews and classroom observations. Bringing everything together, I then discussed the findings using the ROAD-MAPPING theoretical framework and I concluded by explaining the contribution of this study to the knowledge bases of how digital technologies are used in this EMI environment. During the whole research process, I managed to conduct those stages rationally to establish the trustworthiness and ensure the ethical standard of the study. 


\subsection{Trustworthiness}

It is critically important for qualitative researchers to establish the trustworthiness of their research (Williams \& Morrow, 2009). In ensuring rigour in my study, I include a clear rationale, details of the data collection procedures, data analysis methods and the interpretations of findings (Choudhuri et al., 2004). Specifically, I follow Lincoln and Guba (2007) criteria for trustworthiness which include credibility, dependability, confirmability and transferability (Houghton et al., 2013).

\subsubsection{Credibility}

Credibility is one of the most significant factors in building the trustworthiness of the research (Lincoln \& Guba, 2007). It refers to the true value of the data about the participants' views or the accuracy of researchers' interpretation or representation of the participants' experiences (Polit \& Beck, 2016). To enhance the credibility in my study, I clearly demonstrated my prolonged engagement with the participants by writing detailed descriptions of my research experiences as well as the research methods (Cope, 2014). I spent sufficient time in the field to gain a full understanding of the phenomenon. Observations, focus groups and individual interviews were conducted with participants in all cases to ensure sufficient, appropriate data (Casey \& Murphy, 2009) and I employed the strategy of member-checking to ascertain the accuracy of information from respondents and therefore enhance credibility (Stake, 2005). Teacher participants were asked to review the interview transcripts for further adjustment or modification, and student participants were asked to check the summary of their focus group discussions. All participants were encouraged to contact me with any queries concerning the data, a crucial consideration in strengthening a study's credibility (Denzin \& Lincoln, 2018).

\subsubsection{Dependability}

Dependability refers to the stability of the data collected (Rolfe, 2006). This means that the study's findings will be the consistent if another study is conducted in similar conditions with similar participants. To achieve this, I comprehensively described the methodology of the study to make sure that the procedures can be repeated (Shenton, 2004). In addition, I applied the same process consistently to all cases in the research. In the data analysis phase, I conducted a code-recode to "increase the dependability of the study" (Krefting, 1991, p. 221). I also used expert colleagues to back-translate from English to Vietnamese. 


\subsubsection{Confirmability}

Being closely linked to dependability, confirmability implies the neutrality and accuracy of the data (Tobin \& Begley, 2004). By applying reflexivity in considering my role as a researcher of the study, I minimised the influence of my personal biases and viewpoints to ensure that the data collected represented participants' experiences and opinions (Polit \& Beck, 2008). I tried to consider my research within the current social circumstances and to clarify the research process and my role at every stage by keeping a research journal with reflective notes. This also helped me fully acknowledge "beliefs underpinning decisions made, methods adopted as well as the reasons for favouring one approach" designing the study (Shenton, 2004). In addition, I maintained an audit trail (Guba, 1981), by working step-by-step with my supervisors to justify the decisions I made throughout the research process (Shenton, 2004).

\subsubsection{Transferability}

Transferability is defined as the possibility of applying research findings to different settings or groups of participants (Houghton et al., 2013). To meet this criterion, researchers are required to provide readers with adequate information about the participants and research context so that readers can determine whether the results are transferable or not (Cope, 2014). In my study, thick description (Guba, 1981) was used to convey the findings of the study to make the results more realistic and rich (Creswell, 2018b). This description included accounts of the context, examples of raw data such as direct quotes of participants or excerpts from field notes (Houghton et al., 2013). Detailed and appropriate descriptions enhanced the transferability of this study.

\subsection{Ethical considerations}

In educational research ethical issues must be considered throughout the research process (Creswell, 2018b). My study was given ethical approval (N. 24822) by the Victoria University of Wellington Human Ethics Committee. Some ethical issues under consideration included gaining informed consent, protecting privacy, maintaining confidentiality, and building trust.

I addressed these issues by beginning with informed consent. I provided all participants with information sheets and the opportunity to ask questions about my study. I sought and gained informed consent from all participants and stakeholders when starting the process of data collection.

Participants' privacy was considered at all stages of the research. Teachers and students were sent the interview questions in advance so that they felt comfortable. They were informed of their right to refuse to answer any questions and students were asked to discuss and agree on the ground 
rules for focus group discussions themselves. I asked for participants' permission to audio-record the interviews and video-record the classroom observations. Students were told about the time of classroom observations even though the teacher was the focus of the videos, and not them.

Confidentiality was maintained during the research. The identity of the university and schools was not mentioned in any documents of the research and the teacher participants were given pseudonyms so that they could not be identified. These names were approved by the teachers themselves. Focus group students' names were not used in any reports or documents. Instead, they were numbered. Data transcripts and field-notes were stored and managed in secure, confidential folders and all data will be destroyed one year after the conclusion of the research.

Further, I spent time establishing trust with the participants. I arranged time for personal meetings with the teachers so that I could talk to them about my study and invited them to share their feelings and opinions. This helped me develop a good rapport with the teachers, which made them feel secure about participating in interviews. I also spent time talking to students and listening to them at the beginning of the focus group interviews. I sent the participants transcripts of interviews, field-notes, and summary of focus group discussions for member checking.to ensure that I could accurately present the participants' experiences and perspectives.

\subsection{Limitations of the study}

This study has some limitations. First, it involved one HE institution in Vietnam and a limited number of participants. Thus, this small sample is not designed to be representative of EMI in Vietnamese higher education and the study's findings are unlikely to be generalised. However, the purpose of case study research is not to provide generalisation, but insights into the particular nature of a phenomenon. In this study, the particularities centred on EMI and the use of digital technologies by teachers and students in one Vietnamese context. Second, the classroom observations were conducted once in each case, which could not capture all the participants' practices, yet did offer a snapshot of the class on that day. Moreover, the classroom practices might have been affected by the presence of the researcher and the filming activity. Despite different sources of data from teacher interviews and student focus group discussions that allowed for triangulation, the data reflecting classroom activities might be considered limited. Finally, the video stimulated recall interviews were not conducted consistently. Only three teachers agreed to be filmed during the classroom observation; the video recordings did not include students' activities (for ethical reasons); and two interviews were conducted two weeks after the classroom observations to accommodate the 
teachers' busy schedules. Those issues might have influenced the data even though I used field notes during the interviews to complement the data I gathered.

\subsection{Chapter summary}

This chapter thoroughly describes the methodological approach used to explore the teachers' and students' experiences in EMI courses and their use of digital technologies in teaching and learning activities at a Vietnamese university. I used qualitative case studies to present in-depth information from the participants through semi-structured interviews, classroom observations, and focus-group discussions. The data collected was thematically analysed with reference to the research questions and literature, and the results are reported in the following two chapters. Chapter 4 provides detailed descriptions of the four cases covering the context, the teacher, their content teaching, their use of language and digital technologies, and the students from each class. Chapter 5 presents four themes

from the cross-case analysis: An expectation - reality gap; Pedagogical decisions; Integration of content, English and digital technologies; and Developing autonomous learners. 


\section{Chapter 4. Single Case Descriptions}

This chapter provides detailed descriptions of the four cases conducted in the study. Each case was constructed from the data collected through interviews with the teacher, the classroom observation, and the student focus group. Each case description is divided into three parts. The first part provides information about the teacher and their educational context during the data collection period. In each instance, I described the teacher's experiences and beliefs as a way to explore their perspectives on factors affecting their teaching practices. The second part focuses on the teacher's activities to illustrate their approaches to teaching content knowledge, the use of English as a medium of instruction, and the use of digital technologies in teaching. The final part describes the students' learning in an EMI environment, and their use of digital technologies in learning activities.

\subsection{Case 1 - Vincent}

The first case involved one teacher and seven students in an EMI management course. Two teacher interviews were conducted at the beginning of the nine-week course, the first of which took place in the first week, and the second in Week 3. A classroom observation was conducted in the second week, and the student focus group discussion was scheduled in the third week (alongside the second teacher interview). Being new to teaching and learning through English, both the teacher and students had their own concerns and were trying to adapt to the new context.

\subsubsection{The context}

The school within the university where Vincent worked offered academic programmes at bachelor, master, and doctoral levels majoring in management. At the bachelor level, it offered specialties related to different areas of business administration. These specialties were at the beginning stages of implementing EMI programmes. Vincent was an academic staff member in one of the six divisions in the school.

The High-Quality Programme to which Vincent was first assigned included EMI courses in economics subjects. Vincent volunteered to take over one class when there was a shortage of teaching staff in his division. There were six lecturers in his division but only one lecturer was able to teach in English. This lecturer used to study in the Netherlands and had good English proficiency, but he felt overwhelmed by the number of classes he had been asked to teach.

The class Vincent took over included 41 third-year students who had passed the foundation subjects and were starting to study in their area of expertise. Those students had taken one subject 
in English the previous term and were taking three in the current term. They had completed four modules of English and were working on the last two optional ones. The main course book was written in English and due to its high price students accessed a photocopied and scanned version. Content from selected chapters in the book contributed towards three subject credits. Vincent allowed students to use their own laptops and mobile phones in class for looking up difficult words, taking notes or searching for information.

\subsubsection{The teacher}

At the time of the study, Vincent had been working as a lecturer at the university for nearly five years. He had three bachelor's degrees, one of which was in English Linguistics. He also held a master's degree in Business Administration and was preparing to defend his PhD thesis in Management. These qualifications and four years' experience of teaching at the university provided him with confidence in his teaching skills. He also felt confident with his content knowledge having studied at both bachelor's and master's levels.

Vincent participated in the EMI programme because he wanted to challenge himself and adapt to the new situation in response to the trend of internationalisation in education. However, despite having a bachelor's degree in English, Vincent was conscious that he did not have any experience in using English to teach. He was well aware that that he was the only participant teacher who had not completed the teacher preparation for teaching EMI and that he had taken over the EMI class even though he had not attended the training. He was not sure if he could communicate clearly and precisely the objectives and subject matter to his students. He was not only concerned about making himself understood in class, but also about being less articulate in expressing ideas and arguments than if he was using Vietnamese. Despite these concerns, he believed that he could benefit from teaching the EMI course:

When transferring into using English to teach, I have to face challenges from which I can learn a lot. I can learn how to make students understand, and how to adapt to different students in different classes. I often get some students to give me feedback after each lesson to know my weaknesses.

Vincent felt confident in his ability to use a wide range of digital technologies in his teaching. He believed that he "could easily learn how to use software, hardware or applications from the Internet" and was aware of the benefits of integrating digital technologies into his teaching practice, as evidenced in the following quote: 
Using technologies helps me access reference materials to make my lectures more exciting to students. I can engage students better into the lessons and encourage them to use digital tools to assist their learning both in class and at home.

He used a number of web-tools or visual applications in preparing and giving lectures, and social networks to communicate with both colleagues and students.

Vincent's teaching philosophy was to support students in understanding the subject content and in finding ways to achieve good results in their study. He kept seeking ways to improve his strategies for teaching in English to help students overcome difficulties in learning through English. He talked to them quite often about which activities would be appropriate and effective in class. He provided students with resources and tools such as links to relevant journals, electronic books as well as business dictionary software or English-study applications. However, he wanted students to demonstrate that they were active in learning, stating that "What students need first is a willing-tolearn attitude. I accept their mistakes in English or using of Vietnamese as long as they feel comfortable and motivated to study."

In summary, Vincent believed he had strong content knowledge and sound experience in teaching his subject in Vietnamese. He was also confident in his ability to use digital technologies in his teaching and to support students' learning. However, he had concerns about using English to give instructions and explain content knowledge to students. This prompted him to make an effort to improve his teaching of content knowledge, the use of English, and the use of digital technologies.

\subsubsection{Teaching content knowledge}

Vincent taught some subjects related to Business Management. He was confident in his subject content knowledge because of his qualifications and years' teaching experience in the field. He believed that he had been "successful in teaching the subject in Vietnamese". He knew what to teach and proactively planned the content of the course, as explained below:

I needed to identify what objectives were necessary for students at bachelor level. They were only required to understand the foundation theories and apply them in practice. I would show them how different the Vietnamese context was from the world context.

He told me that teachers like him had to manage an overwhelming syllabus within tight time constraints as "the syllabus also included too much content and [he] had to teach two chapters a day". He was aware that teaching content in another language would take more time, which required him to be flexible in his teaching approach. 
Vincent used an array of teaching and learning activities in class. To teach business concepts, he chose the approach of lecturing the students and providing them with "practical examples related to the Vietnamese context" that were easy for students to understand. What he wanted was to help students thoroughly understand the theory so that they could apply it in the Vietnamese context. He gave an example of getting students to read the case of Nigeria and compare it with Vietnamese context. He also found ways to facilitate students' learning in class by using case studies and group work:

I often use video cases for students to discuss. Opening cases help students identify some issues related to the theories that they are going to learn. Closing cases are exercises in which students have to apply the theories to solve specific issues.

He strategically used case studies for different purposes at different stages of the lessons. He got students to work in groups and prepare the cases at home so that they could discuss them in class and check their answers. He supported students by showing them "how to summarise the cases in English so that they could comprehend the texts to pick out main points for discussions or debate in class". He believed that students could gain new skills and engage by learning the content in English.

He also designed games with PowerPoint templates and played songs from YouTube as classroom warm up activities. He thought these would help entertain students, raise their curiosity about the content of the lessons, and "create a secure learning atmosphere in class for students not to be nervous". Vincent often took 15 minutes at the beginning of each lesson for review and reflection. He asked short questions in English that enabled students to recall "key words from previous lessons" and allowed him to check students' comprehension. However, Vincent thought that language was a barrier to interaction in his class and he was not satisfied with the students' passive mode of learning, as evidenced in the following quote:

During my lectures, students mainly sit in class looking at the slides in which I summarise key points. They rarely ask questions and appear to be reluctant to answer my questions as well. They are only passive recipients of knowledge.

This explains why Vincent kept changing how he communicated the economic concepts to students. His aim was to "have a mutual interaction in class where students would actively identify key information by themselves and discuss with each other to decide the main ideas". The teacher would then offer to them help by summarising and consolidating targeted knowledge.

Vincent had some difficulties in planning lessons as he lacked materials. Vietnamese lecturers usually follow the lesson plans from a course book. However, he did not have a teacher's book or the 
original course book and neither was he provided with video cases, slides or answer keys for the lessons. He said, "Photocopying some sections in the book is a way to deal with the copyright, but pictures and words are unclear and unattractive". Thus, the limitations of the teaching materials affected his teaching. Moreover, it took him time to prepare for the lectures as he "had to depend on his own experience and knowledge". To deal with this, he had to learn from experienced teachers by taking some short training courses on teaching methodology. As those courses were in Vietnamese, he aimed to look for strategies which were likely to be appropriate for his EMI class.

A further concern was about the quality of the test papers as they had to be written in English and the normal practice was to use published materials. Vincent believed that teachers must be careful in designing test papers and assessing students as "if the tests are too easy or too difficult, they affect the assessment process". While the university had not purchased the test bank for EMI teachers to use, he was fortunately able to borrow the published tests from his colleagues teaching overseas.

I observed Vincent conducting a range of teaching activities that aimed to help students achieve different course objectives (see Table 6).

\section{Table 6}

Classroom observation (Vincent): Summary of teaching steps

\begin{tabular}{|l|l|l|l|}
\hline No. & Teaching steps & Activities & Objectives \\
\hline 1 & Warm-up & $\begin{array}{l}\text { Games: students passed a ball to others } \\
\text { while a song was playing. The teacher } \\
\text { stopped the music, the student holding } \\
\text { the ball had to answer a question in } \\
\text { English. }\end{array}$ & $\begin{array}{l}\text { - To wake students up in the } \\
\text { morning. } \\
\text { - To help students recall key } \\
\text { business concepts as well as key } \\
\text { English words. }\end{array}$ \\
\hline 2 & Lead-in & $\begin{array}{l}\text { Opening cases: A case was assigned to } \\
\text { students at the end of the previous } \\
\text { lesson. Students worked in groups and } \\
\text { answered the questions related to the } \\
\text { case. }\end{array}$ & $\begin{array}{l}\text { - To raise some practical issues } \\
\text { related to the theory that can be } \\
\text { applied to solve problems. } \\
\text { - To help students identify the } \\
\text { issues, not solving them. }\end{array}$ \\
\hline 3 & Presenting new & $\begin{array}{l}\text { Lecturing: the teacher provided } \\
\text { concepts }\end{array}$ & $\begin{array}{l}\text { - To provide theory related to } \\
\text { concepts and practical examples } \\
\text { relevant to different contexts with a } \\
\text { particular focus on the Vietnamese } \\
\text { context. }\end{array}$ \\
\hline
\end{tabular}




\begin{tabular}{|c|c|c|c|}
\hline 4 & Practice & $\begin{array}{l}\text { Closing case: Groups of students were } \\
\text { asked to solve a problem in a previously } \\
\text { assigned case. They had to make a } \\
\text { short presentation summarising the } \\
\text { case and providing their solutions to } \\
\text { the problem. All groups had to work on } \\
\text { the case as the teacher would randomly } \\
\text { select a group to present. The activity } \\
\text { was followed by a whole-class } \\
\text { discussion. }\end{array}$ & $\begin{array}{l}\text { - To have students practise } \\
\text { applying theory and problem } \\
\text { solving. }\end{array}$ \\
\hline 5 & After class & $\begin{array}{l}\text { Open talk to students ( } 15 \text { minutes): The } \\
\text { teacher chatted with some members of } \\
\text { the class. He asked students for their } \\
\text { feedback on his teaching strategies and } \\
\text { checked their comprehension of the } \\
\text { lesson. } \\
\text { Students asked for clarification about } \\
\text { the topic, asked about points they } \\
\text { found unclear, and gave him some } \\
\text { suggestions about the use of English } \\
\text { and Vietnamese, the blackboard and } \\
\text { mind maps, and pointed out some } \\
\text { students who could not follow the } \\
\text { lessons in English. }\end{array}$ & $\begin{array}{l}\text { - To find out students' problems so } \\
\text { that he could adjust his teaching } \\
\text { approach to help students achieve } \\
\text { the lesson objectives. }\end{array}$ \\
\hline
\end{tabular}

The observation was useful in understanding students' classroom activities. The students sat in their own groups and they used Vietnamese to talk to each other and to discuss the teacher's questions. Those who were randomly selected answered questions in English to the whole class. Some students used laptops to take notes or read slides and e-books and many used their mobile phones in class for looking up words, reading slides or accessing information. Three groups were chosen to present the study cases, one of which used slides during their presentation. All speakers used their mobile phones to access their notes when presenting in front of the class.

In summary, Vincent was confident about teaching content in Vietnamese. He believed that English was a barrier, so he tried to use different teaching strategies such as case studies, group work, presentations and lecturing. Because the syllabus was so extensive, he focused on explaining business concepts through practical examples and having students memorise key English words. He wanted to increase teacher-student interactions as well as student-student discussions in class. He was 
concerned that the shortage of materials added to the time and effort he needed for preparing lessons and working on assessments.

\subsubsection{The use of English}

Vincent had not taught in English before though he reported that he had been a confident student pursuing his bachelor's degree in English. He had registered to take an EMI course at the university to prepare for this teaching as he considered it as an opportunity to challenge and improve himself, but there were not any courses available for him at that time. Teaching economics in English was a challenge for Vincent. He found it hard using English to express opinions and communicate the content to students. The linguistic problems he identified were his limited English vocabulary and concerns about making grammatical mistakes. He reported, "There are a lot of new words I have never met before, so I can easily mispronounce them if I do not prepare carefully". Facing difficulties in using English, Vincent spent a lot of time preparing what he was going to say and how he was going to say it in class, as summarised below:

It often takes me two days to prepare for a three-hour lecture in class. I have to think of what I want to say about key points in slides, then write it in English and learn it by heart. If the topic is easy, I can remember the English very quickly. This helps me able to express the content fluently and accurately; otherwise, there will be trouble.

This also happened when he prepared case teaching activities in which he tried to understand video cases without subtitles so that he could know "where to ask questions and what the answers are".

Vincent used a lot of English in his lectures. He extended the key English words in slides to make full sentences and communicate the concepts to students. Most instructions were also given in English. He did not feel confident in his use of English as he believed that if his English had been better, he could have made sentences using simple and comprehensible words. To teach students the business concepts, he often used the definitions in the course book despite his belief that this was not an effective means of teaching and that more experienced teachers "were able to do it better". Believing that subject definitions in English were complicated and too difficult for students to understand, Vincent sometimes simplified the terms. He gave the following example: “[ ...] talking about globalisation, the key words are integration and mutual dependability. I have to use Vinlish to make them comprehensible". He meant the kind of English which is influenced by Vietnamese and popular in spoken language of daily conversations.

Vincent assumed that both policy makers and students expected him to use English in EMI classes. He tried to use as little Vietnamese in class as possible. He explained that he wanted students 
to have frequent exposure to English. He only used Vietnamese as a refocus tool when he recognised that students had stopped engaging with the lectures in English. He explained, "I realised that some students, especially those in the corner, appeared to be bored and distracted when listening to long lectures in English". He believed that his competence and classroom experience would help him flexibly select appropriate languages. He reported, "During the lecture, I had to interact and observe students to know their attitudes and responses. This is the teacher's classroom management skill." In addition, when dealing with challenging content, he admitted having to use Vietnamese:

When I talked about two monetary policies, I thought that students might not understand them in English, so I used Vietnamese almost entirely, and then added some key words in English. I had to 'sacrifice' to use Vietnamese as this knowledge is related to Microeconomics, Macroeconomics and Monetary Finance, which are very difficult for students to remember.

In this example, he depended on his own experience of learning in English to identify the difficulties that students might encounter in studying content knowledge. He thought that English should have been used in EMI classes, so he considered his use of Vietnamese as non-compliant with but helpful for students.

Vincent had difficulties working with students who had different levels of English proficiency. He described his class as being a mix of three groups of students. The first group included students sitting at the back of the classroom. They seemed unable to understand the lessons in English and have limited competence in English. The second group included students who could follow $50 \%$ of the content in English and the last group included students with high levels of English who could easily follow the lesson in English. The issue was how to make sure that the whole class could achieve the lesson objectives. Vincent learnt from the students that they were used to listening to teachers using both languages in class. This meant that the teacher immediately translated a point in English into Vietnamese. However, Vincent decided to use Vietnamese to summarise the knowledge after each section of the lectures: "If I use English all the time, many students who cannot understand will be demotivated and quit studying. This will affect their attitudes and study results". He wanted all students to understand and believed that "teachers have to adapt to meet the demands of all groups of students, especially to make the weakest ones understand'. He also managed to find ways to enrich interactions with students in class and encouraged them to contribute by allowing them to use both English and Vietnamese. 
Vincent reported that he did not set any language objectives for his lectures. He had neither intention of nor experience in teaching English. However, his teaching activities were consistent with several principles in content-based language teaching (see Table 7). He used games and music to create a relaxing atmosphere for students to study his subject through English and use of group work helped students to apply what they had learnt in the past and link this to new subject matter to solve problems. The objectives set for content learning showed that Vincent focused on academic content and expected students to understand all subject matter. Vincent paid attention to academic language by providing students with definitions and explanations for all business concepts but knew little about the language demands in his subject. Group-work activities and presentation tasks offered students opportunity to engage in interactions where they could improve their communicative skills. Although these activities did not seem as effective in enhancing students' English competence as Vincent and his students expected, they increased students' exposure to English as a medium of instruction. 


\section{Table 7}

Vincent's teaching activities compared to content-based English teaching principles (Gleeson, 2015).

\begin{tabular}{|c|c|c|c|c|c|}
\hline Activities Principle & $\begin{array}{l}\text { Knowing how } \\
\text { English is learnt } \\
\text { through content }\end{array}$ & $\begin{array}{l}\text { Creating } \\
\text { connections } \\
\text { between the } \\
\text { students' } \\
\text { experiential } \\
\text { background and } \\
\text { new ideas }\end{array}$ & $\begin{array}{l}\text { Sustaining } \\
\text { academic rigour } \\
\text { and maintaining } \\
\text { high expectations }\end{array}$ & $\begin{array}{l}\text { Knowing the } \\
\text { academic language } \\
\text { demands of the } \\
\text { subjects and } \\
\text { sustaining a focus } \\
\text { on academic } \\
\text { language }\end{array}$ & $\begin{array}{l}\text { Engaging } \\
\text { students in } \\
\text { quality } \\
\text { interactions that } \\
\text { promote } \\
\text { productive and } \\
\text { receptive skills }\end{array}$ \\
\hline $\begin{array}{l}\text { Using video cases as the basis for } \\
\text { group work to answer given } \\
\text { questions in the case. }\end{array}$ & & $\checkmark$ & $\checkmark$ & & $\checkmark$ \\
\hline $\begin{array}{l}\text { Having students present in groups to } \\
\text { solve the problems in the video } \\
\text { cases. }\end{array}$ & & $\checkmark$ & $\checkmark$ & & $\checkmark$ \\
\hline
\end{tabular}


In summary, Vincent had concerns about using English to teach in class. He thought that his limited vocabulary and language competence were barriers and spent extra time before class preparing how to express lecture content in English. He tried to speak as much English as possible in class but confessed that he switched into Vietnamese to deal with difficult content or to make students stay focused. He prioritised students' comprehension of content knowledge over teaching exclusively in English as he recognised the difference in students' English proficiency. Although he focused students on improving business vocabulary and exposure to English, he had not learnt how to teach in English, so he was not aware that there might be language demands in addition to vocabulary.

\subsubsection{The use of digital technologies.}

Vincent used a variety of digital technologies to support his teaching and students' learning (see Table 8). He described himself as a teacher who was able to use technological equipment and was confident in his ability to learn to use new technologies. The only resources he needed were "a good laptop and relevant software to process video clips, or simply a better one than [his] current one so that [he] could download English or Vietnamese subtitles for the clips".

\section{Table 8}

Vincent's use of digital technologies for teaching (Starkey, 2011)

\begin{tabular}{|c|c|c|c|c|c|c|c|c|}
\hline \multirow{2}{*}{$\begin{array}{c}\text { Digital } \\
\text { technology }\end{array}$} & \multicolumn{3}{|c|}{ The Internet } & \multicolumn{2}{|c|}{ Social network } & \multirow[t]{2}{*}{ LMS } & \multirow[t]{2}{*}{ PowerPoint } & \multirow{2}{*}{$\begin{array}{l}\text { Computer } \\
\text { software }\end{array}$} \\
\hline & Websites & Google & YouTube & Facebook & Blog & & & \\
\hline $\begin{array}{l}\text { Accessing } \\
\text { information }\end{array}$ & $\checkmark$ & $\checkmark$ & $\sqrt{ }$ & $\checkmark$ & & & & \\
\hline $\begin{array}{l}\text { Presenting } \\
\text { information }\end{array}$ & & & $\sqrt{ }$ & & $\checkmark$ & & $\checkmark$ & \\
\hline $\begin{array}{l}\text { Processing or } \\
\text { creating digital } \\
\text { objects }\end{array}$ & & & & & & $\checkmark$ & & $\checkmark$ \\
\hline $\begin{array}{l}\text { Gaming or } \\
\text { interactive } \\
\text { programmes }\end{array}$ & & & & & & & & \\
\hline $\begin{array}{l}\text { Communicating } \\
\text { or collaborating }\end{array}$ & $\checkmark$ & & & $\checkmark$ & $\checkmark$ & $\checkmark$ & & \\
\hline
\end{tabular}

Vincent used PowerPoint slides in class as he was able to "download those slides from different open sources on the Internet". Yet he had to adjust and modify them so that they fit his content teaching objectives. Slides were normally used to summarise key points from the lectures, so students were able to follow them. He thought that slides were effective and convenient as he 
could add "examples, visual aids like pictures, clips, graphs or charts." However, he said that using slides alone was not effective in ensuring that all students understood the content, especially those students with low English proficiency. In his opinion, students could not follow the information on slides and listen to teachers at the same time, especially in English. That was the reason why he had to "simplify the slides, use them as a tool supporting teaching, explain the lessons and write on the board, which seems very effective." In this sense, he used slides with key information "to give students a consolidation of what they need to note down."

YouTube was one of the main sources that Vincent used for video cases. These videos were chosen as an overview of the business situation or to offer theory-related examples. He often got students to watch the video directly on YouTube so that they could see the English subtitles. However, he found it challenging to access the clips he needed:

Video cases are not provided, so I have to download them from YouTube. The weak point of YouTube is that we cannot watch clips with subtitles directly unless the Internet access is strong. If only I was supported with video cases and their scripts.

This, accordingly, required him to spend more time preparing for lectures.

Vincent was also familiar with getting students to use the LMS, a form of e-learning that had been applied at the university the year before. He used the LMS as a channel for students to selfstudy at home. He uploaded homework, test formats, assessment criteria as well as scanned books as resources. He used the LMS in all of his lectures., as detailed in the following quote:

E-learning is now compulsory at our university. Each subject with 45 periods has to include at least two lectures online. Instead, I use it for eight lectures by posting questions for student discussions. I post the syllabus and materials on eight different topics.

To support this system, he integrated additional software supporting students to submit assignments in English. He "showed them how [he] would mark their answers as well as some software used to check their assignments like Grammarly and Turnitin." A forum function was also available but Vincent did not see it as very effective because it was slow and not user-friendly.

Vincent used digital technologies to "encourage students to use digital technologies to support their study". He had a BlogSpot in Vietnamese where he uploaded his research and writing on the subject and students had access download what they needed. He contacted students through Facebook and found that this social network was an effective way to exchange information thanks to its coverage and speed. This was how he became close to his students and was able to develop 
rapport with them. He believed that "Facebook is a wonderful channel to exchange information, which can be effectively used to support studying".

Vincent used networks for his professional development. He joined different forums and specialised websites in Management so that he could learn from foreign professors and widen his knowledge. He also took some short courses in English online through Facebook to improve his use of English, and was a member of an online linguistics group, which helped him learn about English.

In summary, Vincent was familiar with using digital devices in his teaching. He employed multimedia equipment in presenting his lectures, integrated technologies through the e-learning system, communicated with students through social networks (Facebook, blogs), provided students with reference material, and set up opportunities for students to interact with materials online.

\subsubsection{The students}

Seven students - three males and four females - in the class participated in a focus group discussion. They expressed different levels of confidence in three areas of content knowledge, English competence and digital technology (see Table 9). All of them reported familiarity with using digital technologies for both learning and everyday activities. They were experienced in using Office software like Word, PowerPoint or Excel as well as Internet-based tools for social networking. Two students believed that they were capable of learning subject matter and felt confident with their level of English proficiency (Student 2 and Student 3). Three others said that they could use English for communicative purposes, but had some difficulties learning in English (Student 1, Student 5, and Student 6). The last two described themselves as not confident in their language competence and admitted to struggling with English (Student 4 and Student 7). Yet these five students confirmed that they had sound content knowledge.

\section{Table 9}

A summary of students' profiles regarding their confidence from the most (1) to the least (3)

\begin{tabular}{|l|c|c|c|c|}
\hline \multirow{2}{*}{ Students } & \multirow{2}{*}{ Gender } & \multicolumn{3}{|c|}{ Field of confidence } \\
\cline { 3 - 5 } & & Content knowledge & English competence & Digital technology \\
\hline Student 1 & Female & 1 & 2 & 3 \\
\hline Student 2 & Female & 2 & 1 & 3 \\
\hline Student 3 & Male & 3 & 1 & 2 \\
\hline Student 4 & Male & 2 & 3 & 1 \\
\hline Student 5 & Female & 1 & 2 & 3 \\
\hline Student 6 & Female & 1 & 2 & 3 \\
\hline Student 7 & Male & 2 & 3 & 1 \\
\hline
\end{tabular}


Four out of seven students perceived benefits of studying economics in English, expressing a clear preference for studying in English rather than Vietnamese. Students with high English competence believed it would help them improve both content knowledge and English. They anticipated that they would be offered more opportunities in their future career, especially when they wanted to "have a job in a foreign company". The following quote from Student 3 is representative of comments about the perceived benefits to their English:

Studying this subject in English helps me practise how to think in English, which is different from Vietnamese. For example, when writing essays, instead of taking time to think in Vietnamese and translate into English, I can think and write in English.

Even students who reported having little confidence in English felt motivated in their study, as they had to make an effort to overcome the language challenge. They thought that it would help them "stay more focused to prepare for lessons better", as evidenced in the following quote:

Studying in English has made me busier. I have to learn at my top productivity. Despite that, I have been able to do more things than in previous semesters. However, my content knowledge is still limited, so I need to try harder. I feel delighted.

On the other hand, three students said that working in English put them under too much pressure. Although they had confidence in their content knowledge, they felt that English was a barrier hindering their content learning. Being third year students, they had to deal with a great deal of intensive and abstract knowledge in their major subject. Student 7 said:

Studying economics in Vietnamese has been tough, so it is tougher in English. I do not know how to process an overwhelming amount of content. It always takes time to find extra materials, so I have to triple my time investment into the subject. Third-and fourth-year students are under pressure handling tough content. I think this programme should start earlier in first years.

This suggested that students had not been well prepared for the courses. They were not familiar with processing content in English, as they even found this hard to do in their mother tongue.

Students had to do prepare for lessons in advance at home, as they did not feel confident about learning in class. They commonly tried to read all of the concept definitions and examples to understand them then identify the key words to gain an overview of the lessons. Students good at English mostly handled these steps in English apart from unfamiliar key words that they needed to "look up in the dictionary for their meanings and synonyms".

Meanwhile, students who had concerns about both English and content tried to make the content understandable. These students reported using software to translate the classes so they 
could understand and memorise content knowledge in both languages, as expanded on by Student 5:

I read definitions, using mind maps to combine them. To memorise a concept about a business activity, I tried to link its operation and roles together in both English and Vietnamese. I used Google to translate the concepts into Vietnamese and remember them in Vietnamese, and then I express my understanding in English.

Thanks to such preparation, they were able to follow the lessons and felt motivated by the learning activities that they were assigned.

Regarding group work activities, all students liked studying with case studies as they saw these as offering good preparation in both content and language before the class discussions. They found it useful to explore case studies carefully at home to have the answers. However, one student complained that "some cases are not closely linked to the content of the lessons, so the solutions to the cases are not clear".

There was a down-side to case study as some students revealed that they only focused on what they had prepared, and not on what their friends presented in class. They waited for the answers from the teacher at the end of the discussion but found it too challenging to follow their friends' reports in English, as evidenced by Student 4 below:

My friends often write their answers and read aloud in class. Their pronunciation is not clear and precise enough for me to understand. I only compare my answers with the teacher's. If there are slides, I prefer to read key words on slides.

They stated that this was the reason why they did not discuss content or interact with friends in English. Moreover, when assigned to make a class presentation, each student only read the section he or she was responsible for. They could not understand the whole lesson but spent time working on the other sections outside class.

The students had some concerns about Vincent's teaching. They recognised that he was under pressure from an overwhelming content load, but they found this made him speak too fast and skip detailed information. Student 3 explained:

The teacher had to go through two chapters on that day, so he was in rush. He only went over the definitions, but not explaining and giving examples. I only remembered what I had prepared at home. Too much content knowledge makes learning activities ineffective.

Further, the students sometimes struggled to understand their teacher's use of English. Even though his use of video cases motivated them to learn, his limitations in using content English inhibited him from explaining complex concepts. One student complained about the teacher's 
mistakes in pronouncing words. Another student added that the teachers copied exactly what was written in the book when explaining the concepts, and used many linking words, "which made [her] confused and it was difficult to understand his points". On these occasions, some students said that they would have preferred a native English-speaking teacher whose intonation made it easier for them to follow the lesson.

In terms of the course objectives, the students assumed that this subject is aimed to improve their content knowledge and English skills. Yet, they did not feel confident that they would achieve those objectives as the classes mainly focused on teaching content knowledge, which was considered the major objective. In fact, English was often a barrier that hindered them from understanding the lessons, as mentioned by Student 3 in the following quote:

I think that the objectives of this subject cannot be achieved as English is difficult and our levels of English in class are different. Some of my friends focused on studying content knowledge by translating it into Vietnamese. Others considered this subject as an English module.

Regardless of these criticisms, the students, especially those with high levels of English proficiency, did not want the teacher to use Vietnamese in class. One student explained that many business concepts in English cannot be explained precisely in Vietnamese" and felt that the teacher "should paraphrase with simpler English words rather than using Vietnamese".

The students did not think that they were "able to learn and improve English from the teacher in class", but thought their self-study might have helped, as evidenced in this quote:

I think my content knowledge has improved, but my English has not except my vocabulary. When I prepared the lessons, I had to read a lot of material in English and try to translate into Vietnamese. Thus, I could learn many business words and learn how to explain business concepts in English.

Students were aware of both positive and negative influences from the teacher. They knew that the teacher "always tried to know students' problems", but he did not always succeed in helping them to study better and "be inspired in class".

All students insisted that digital technologies provided indispensable assistance to their learning. They said that laptops, mobile phones and Internet access greatly benefited their study. Using technology saved them a lot of time in finding information, making plans and managing their study schedule. They used a variety of applications to support their language difficulties and access content resources. They believed that social networking and e-learning extended their learning 
beyond the traditional classroom as they could access open sources online, and people in different and international social groups.

Students mostly preferred to use mobile phones in their study. They used different kinds of applications on their mobile phones for language support and data management such as LacViet dictionary, the Oxford dictionary, Dropbox and Google Drive. They also used the Internet as a source of reference materials, as detailed in the following quote:

I often use phone camera to take photos of my assignments and the MINDMAP application to organise and manage study materials. I use Google translation and Google search for business articles or links to electronic books and YouTube video clips on specific topics.

This indicates that the students used digital and Internet-based tools to find a range of supporting materials.

Students said that they also used laptops a lot. They used Office tools like Word, Excel and PowerPoint to do assignments. They were familiar with web-tools like Prezi or Pinterest for creating presentations. With a larger screen, students found laptops were better for surfing websites, reading material and watching online lectures. One student said, "I use my laptop to take notes on the lesson in class, and watch lectures on YouTube", noting that "some experts in foreign universities gave these lectures in English with subtitles". Another student shared the same habit of watching online lectures related to their subject. She also browsed websites for English study like VOA or VNexpress to practise English.

As members of a technologically savvy generation, all students were familiar with social networking. Social networks like Facebook, Twitter, Instagram or LinkedIn were no longer used solely for making friends, chatting and posting status or pictures. Three students confirmed that they used Facebook very often for different purposes, as described by Student 6 below:

I use Facebook to exchange information with my friends in the group. We discuss the assignments and share useful materials with each other. I sometimes chat with a friend who is living in the USA and studying the same major. I prefer reading journal articles and watching video clips about the business world shared on Facebook.

Some students proactively used these channels to study by joining groups in their field of interest, taking online courses, accessing open resources and even contacting specialists for advice, as evidenced by Student 5.

I joined a start-up club on Facebook where members often share their experience in business start-ups. I am also taking some courses about business on Linked-in and Coursera offered by different overseas universities. 
Generally, students believed that using digital technologies was a must for them not only in daily life but also in their learning. They found applications to assist them in learning English and understanding lessons in English. They used online resources and digital devices to access subject content independently, effectively and efficiently.

In contrast to her friends, one student with a part time job believed that face-to-face communication helped her more than using social networks. She said that she often talked to her native-English speaking boss at work and learnt about business from him. Student 3, however, preferred online resources, providing the following reasons:

In some face-to-face courses, when you ask lecturers directly about an issue, they often provide you with general answers. However, online courses offer you various sources of intensive materials from prestigious universities. You can consult other people like course administrators and mentors as well.

This suggests that students sought help from different people by using different networks. To add to this point, four students sought help from group members to resolve difficulties in studying the subject through English. One of them noted that their "group leader often divides the lesson into different parts and assigns them to every member who will read the text and explain to others about it. We can discuss and help each other understand the lesson".

In summary, students participating in the focus group expressed a desire to study economics through English and believed that there were associated benefits. However, they had concerns about understanding the language for challenging subject content in their third year and some were worried about the teacher's limited English proficiency. They chose to depend on self-study at home and outside the classroom and used different kinds of digital technologies to assist their study including electronic devices (laptops, and mobile phones), applications (Dictionary, Google translations, and Mind-maps), web-based resources (YouTube, Prezi, Google Drive, and Dropbox) and social networks (Facebook, Linked-in, and Twitter) (see Table 10). Students commonly asked for help through social communication and cooperation with their friends. 


\section{Table 10}

Students' use of digital technologies in the digital age learning matrix (Starkey, 2011)

\begin{tabular}{|c|c|c|c|c|c|c|}
\hline Aspects of learning & Doing & $\begin{array}{l}\text { Thinking about } \\
\text { connections }\end{array}$ & $\begin{array}{l}\text { Thinking about } \\
\text { concepts }\end{array}$ & $\begin{array}{l}\text { Critiquing and } \\
\text { evaluating }\end{array}$ & $\begin{array}{l}\text { Creating } \\
\text { knowledge }\end{array}$ & Sharing knowledge \\
\hline $\begin{array}{l}\text { Explanation of } \\
\text { aspects of learning: }\end{array}$ & $\begin{array}{l}\text { Isolated } \\
\text { information. } \\
\text { Focus on } \\
\text { completing a } \\
\text { measurable task. }\end{array}$ & $\begin{array}{l}\text { Connecting } \\
\text { thinking. } \\
\text { Simple } \\
\text { connections } \\
\text { made within a } \\
\text { context. Compare } \\
\text { and share. }\end{array}$ & $\begin{array}{l}\text { Develop } \\
\text { conceptual } \\
\text { understanding of } \\
\text { 'big ideas'. }\end{array}$ & $\begin{array}{l}\text { Evaluating and } \\
\text { critiquing to } \\
\text { explore the } \\
\text { limitations and } \\
\text { potential of } \\
\text { information, } \\
\text { sources or } \\
\text { process. }\end{array}$ & $\begin{array}{l}\text { Creativity - } \\
\text { applying ideas, } \\
\text { processes and/or } \\
\text { experiences to } \\
\text { develop a new } \\
\text { reality. }\end{array}$ & $\begin{array}{l}\text { Sharing the new } \\
\text { knowledge through } \\
\text { authentic contexts } \\
\text { and gaining } \\
\text { feedback to } \\
\text { measure value. }\end{array}$ \\
\hline $\begin{array}{l}\text { Accessing } \\
\text { information }\end{array}$ & \multicolumn{4}{|c|}{$\begin{array}{l}\text { Students used Google and web-based tools to access different sources of } \\
\text { information related to their subject matter. Students also accessed the } \\
\text { teacher's blog for relevant content in their subject. }\end{array}$} & & \\
\hline $\begin{array}{l}\text { Presenting } \\
\text { information }\end{array}$ & \multicolumn{6}{|c|}{ Students used PowerPoint or Prezi to present their answers to the study cases. } \\
\hline $\begin{array}{l}\text { Processing or } \\
\text { creating digital } \\
\text { objects }\end{array}$ & \multicolumn{4}{|c|}{$\begin{array}{l}\text { Students used Google Drive, Dropbox or Mindmap applications to organise and } \\
\text { store their data and notes of the lectures. }\end{array}$} & & \\
\hline $\begin{array}{l}\text { Communicating or } \\
\text { collaborating }\end{array}$ & \multicolumn{6}{|c|}{$\begin{array}{l}\text { Students joined groups on social networks (Facebook, Twitter, Instagram) to communicate and exchange content } \\
\text { knowledge. Students also used LMS to contact the teacher and update information or assignments in their subject. }\end{array}$} \\
\hline
\end{tabular}




\subsubsection{Summary}

Both the teacher and students were new to the EMI context. Despite recognising the opportunities presented by the programme, they had to cope with challenges. Vincent said that he lacked both experience and pedagogical knowledge to teach through English and his students struggled with both the content and the language. There was also a difference in the perceived objectives held by the teacher and students. While Vincent's objective was to have students acquire the content knowledge set out in the syllabus, his students wanted to develop both content knowledge and English. Both parties felt overwhelmed by the content of the subject and confirmed that they needed support from different sources.

Vincent made efforts to adapt to the EMI context. Although his content knowledge was strong, he was not confident in teaching through English, which affected his choices of teaching strategies. He tried different techniques such as lecturing or case studies and different activities like group work, presentations or games. He struggled with students' varied, and in some cases, limited English proficiency and tried code switching between English and Vietnamese to guarantee full content comprehension for all. He was confident in his ability to use technology for teaching. He did not integrate technologies into classroom activities much but used them as tools to support his teaching.

The students attending focus group discussions described their experiences in EMI classes. They perceived English as a barrier hindering their understanding of content knowledge, so they tried to find ways to overcome this by preparing for lessons at home, reading textbooks before class and working in groups on assignments. The majority of them focused on key words, looked for synonyms or simple words to paraphrase business concepts. They trained themselves to think in English and would rather the teacher did not use much Vietnamese in class. Those students who were not confident in English chose to translate the content into Vietnamese, used mind maps to organise ideas and tried to memorise the content in both languages. All students insisted on the necessity of digital technologies in their study. They used electronic devices as laptops and smartphones for searching for information, storing data, making presentations and accessing different sources. They employed social networks like Facebook and LinkedIn to interact with others for daily socialising and advice or experience related to their study.

\subsection{Case 2 - Nathan}

The second case involved one teacher and six students in an EMI economics course. Two teacher interviews were conducted at the beginning of the nine-week course. The first interview 
took place in the first week and a classroom observation followed in the second week. The second teacher interview and student focus group discussion were both held in the third week. While the teacher had some experience teaching economics through English, the student participants were all first-year students in their first semester at university and new to learning economics subjects in English.

\subsubsection{The context}

The school where Nathan worked offered academic programmes at bachelor, master and doctoral levels in economics. This school was also responsible for offering foundation courses to students in all majors at the university such as Introduction to Economics, Principles of Microeconomics and Principles of Macroeconomics.

Nathan was also a visiting lecturer at another school in the university offering academic programmes in business and commerce. At the bachelor level, this school ran both the HighQuality Programme and the Advanced Programme in which EMI courses were included. In the Advanced Programme, students had to study all economics subjects in English from their first year.

The class Nathan was in charge of included 35 first-year students in the Advanced Programme. These students had passed compulsory exams in Mathematics, Literature, and English and some optional exams like Chemistry, Physics or Biology to graduate from High School. Then they were admitted to this university programme based on their English score in the High School Graduation Examinations. Thus, students in Nathan's class were expected to have a high level of English proficiency. Students in this class were encouraged to use the LMS to update information about the course and contact the teacher. They were allowed to used laptops and mobile phones in class to support their study.

\subsubsection{The teacher}

Nathan had been working as a lecturer at the university for more than 10 years. He had a bachelor's degree in Economics and two master's degrees in the field of economics. He obtained his first master's degree in a joint programme between a Vietnamese university and a foreign one in which he had to study all courses through English. Then he went to an English-speaking country to gain the second master's degree. At the time of the study, he was working on his PhD thesis in economics. He believed that having studied the subject at both bachelor's and master's levels, he had strong content knowledge and confidence in teaching the subject to students. 
Nathan reported that teaching on the High-Quality Programme was one of his academic duties. He was invited to cover the course due to a shortage of lecturers in the school, and he believed that teaching this course at bachelor's level would support his English skills:

I have not had many chances to speak English in Vietnam, so this course provides me with an environment to use English frequently. The way I spoke English in the first lesson was always different from the subsequent ones. I think I am getting more and more fluent and accurate now.

Nathan felt confident in using English in his teaching. He said that he had no difficulties in explaining business concepts in English or dealing with tough business terms as he had already studied them in English. He believed that he had thorough understanding of the course content as he participated in the translating of the main textbook used in the course from English. However, he thought that using English sometimes affected his teaching, as is evidenced in the following quote:

Sometimes I want to tell a story as an example to illustrate a concept. If I use Vietnamese, it will be easy for students to understand the funny meaning of the story, which might somehow help them memorise the content for longer. I find it hard to do that using English.

This made him think that using English exclusively was not always an effective way to teach.

Nathan used digital technologies to support his teaching. He employed the Office software and applications installed on his laptop to plan lessons, design slides or draw graphs and charts. He was familiar with using different online search tools to access materials for his lectures. Although he wanted to integrate digital technologies into teaching practice, he felt that he did not have sufficient time for that. He said that "the use of technologies to support students' learning at the university was still limited and ineffective" as he thought that the e-learning system was not attractively designed or user-friendly for teachers and students. In his teaching, he encouraged students to be active and develop autonomy in their learning. He was aware of firstyear students' difficulties, as stated in this quote:

My students are new to university environment. They have to start studying economics subjects without any preparation about background knowledge or materials for reference. They have yet to make friends so that they can study in groups or help each other.

Thus, he said that he had spent time at the beginning of the course giving them advice on the use of online resources and the benefits of group work so that they could quickly familiarise themselves with the new learning environment and context. 
In summary, Nathan felt confident in teaching economics through the medium of English as he had some experience. He believed he had strong content knowledge but found that using English to teach prevented him from expressing himself as effectively and articulately as when he used Vietnamese. Nathan not only used digital technologies to support his teaching and students' learning, but he also wanted to integrate them into his lectures. However, he reported not being able to do that due to time constraints.

\subsubsection{Teaching content knowledge}

Nathan had some experience in using English to teach his subject as he "used to teach undergraduate students and tutor postgraduate students in English". He believed that his teaching experience and strong content knowledge would be beneficial for him in taking over the course, as explained in this quote:

I have studied this subject many times, once at bachelor level and twice at master level, so I am confident that I have a comprehensive understanding of its content. In addition, I have experienced three years of teaching this subject in Vietnamese, working with the Vietnamese version of the textbook.

Nathan followed the syllabus provided by the school and the lesson plans suggested in the instruction manual. He did not have to modify the lesson plans greatly with new ideas, as he found the structure of a lecture was the same throughout the course, beginning with defining concepts, giving examples, then getting students to practice, and finishing at times with "a case study for students to discuss to see how the theory works in practical contexts".

What concerned Nathan was how to explain the business concepts as comprehensibly as possible. He thought the definitions of the terms in the textbook was general enough to be understood by undergraduate students. However, he felt that it was still difficult for students to memorise the concepts. He explained, "In this subject, students need to understand the logic of the matter, so they do not need to learn by heart the definitions but get the key for proper comprehension".

Nathan said he aimed to help students to answer questions or explain real situations in their daily life by applying economic principles. He decided not to use some examples in the textbook, but think of those relevant to Vietnamese contexts, especially those closely related to students' lives. He gave examples:

First-year students did not understand what "inflation" means, so I asked them about their shopping experiences related to price changes over the years. Or, since they had no idea about tax software, I had to find another example like their course enrolment application. 
He thought it was easy for students to understand and remember familiar examples. Additionally, he also used charts and graphs to illustrate the concepts, as he believed they helped students easily understand content knowledge. He believed that "the charts are helpful for students in conducting thorough analysis in later subjects at higher levels."

Nathan had concerns about whether students would understand the subject matter. He realised that as first-year students, they were not familiar with many business terms. He said he had to revise the lessons and ask questions to check their comprehension frequently in class. Further, he decided to set students a mid-term test so that they could "refresh themselves, revise the lessons as well as reset their targets for their next tests".

Nathan felt that both he and the students were under pressure due to the extensive content and had concerns about students' engagement in class due to the challenging subject matter. He sometimes felt demotivated recognising students' tiredness and boredom in class:

I pay attention to students' facial expressions and behaviours in class. When they are unclear about any points, they start to be distracted. When they are overwhelmed, they become tired and lose concentration. I wonder whether I should continue or not.

This seems to reveal the impact of such a full curriculum on both the teacher and students.

Nathan said that there was not enough time to design group work activities for students in class. He believed that group discussions would help students, as they could learn from each other, especially when they were not confident in talking to the teacher. However, he felt that there was no time to conduct group work effectively. He wanted to encourage students to work in groups after class but thought that "first-year students were not involved enough" in group work as they were strange to each other.

During a classroom observation, Nathan conducted a range of teaching activities aimed to help students achieve course objectives (see Table 11).

\section{Table 11}

Classroom observation (Nathan): Summary of teaching steps

\begin{tabular}{|c|c|c|c|}
\hline No. & Teaching steps & Activities & Objectives \\
\hline 1 & Warm-up & $\begin{array}{l}\text { Questions - Answers: The teacher } \\
\text { asked students some questions about } \\
\text { the previous lessons and encouraged } \\
\text { students to ask questions about } \\
\text { anything that was unclear. The } \\
\text { teacher asked for students to }\end{array}$ & $\begin{array}{l}\text { - To help students revise what } \\
\text { they had learnt and recall key } \\
\text { concepts or terms from the } \\
\text { previous lessons. } \\
\text { - To make sure that students } \\
\text { clearly understood the course } \\
\text { content. }\end{array}$ \\
\hline
\end{tabular}




\begin{tabular}{|c|c|c|c|}
\hline & & $\begin{array}{l}\text { volunteer answers or called on } \\
\text { someone randomly. }\end{array}$ & \\
\hline 2 & Lead-in & $\begin{array}{l}\text { Introduction: The teacher provided } \\
\text { students with a brief agenda for the } \\
\text { lecture including content objectives } \\
\text { set for the lecture. For example, after } \\
\text { the second chapter, students should } \\
\text { have been able to understand the } \\
\text { principles of supply and demand and } \\
\text { explain the movement of supply and } \\
\text { demand curves. }\end{array}$ & $\begin{array}{l}\text { - To clearly inform students of } \\
\text { what they were going to learn. } \\
\text { - To raise students' awareness of } \\
\text { the objectives of the lecture so } \\
\text { that they could focus on the right } \\
\text { points. }\end{array}$ \\
\hline 3 & $\begin{array}{l}\text { Presenting new } \\
\text { concepts }\end{array}$ & $\begin{array}{l}\text { Lecturing: The teacher used slides } \\
\text { and a data projector and lectured to } \\
\text { explain the theory and the concepts } \\
\text { and gave examples. }\end{array}$ & $\begin{array}{l}\text { - To transmit the content to } \\
\text { students, ensuring their } \\
\text { understanding of the economic } \\
\text { principles and showing how they } \\
\text { are applied in authentic } \\
\text { situations. }\end{array}$ \\
\hline 4 & Practice & $\begin{array}{l}\text { Group exercises: The teacher } \\
\text { provided students with some } \\
\text { situations on slides and had them } \\
\text { discuss the answers in groups. Time } \\
\text { allowed for discussion was about two } \\
\text { to five minutes. }\end{array}$ & $\begin{array}{l}\text { - To have students apply the } \\
\text { theory they had learnt to do the } \\
\text { tasks by finding solutions to } \\
\text { specific economic situations. }\end{array}$ \\
\hline 4 & Wrap-up & $\begin{array}{l}\text { The teacher randomly selected some } \\
\text { groups to report their answers. Then, } \\
\text { the teacher corrected students' } \\
\text { answers and provided them with } \\
\text { further detailed explanations. }\end{array}$ & $\begin{array}{l}\text { - To consolidate what students } \\
\text { had learnt and make sure that } \\
\text { they could understand the } \\
\text { theory and apply it in practice. }\end{array}$ \\
\hline
\end{tabular}

During the class observation, I noticed that students sitting in the front rows seemed to interact more than those at the back. They mostly focused on their books and slides listening to the teacher. The teacher sometimes called on students to answer questions. The students who came late sat at the back and seemed unable to follow the lesson. They sat in silence with their books but did not ask for help from either the teacher or their friends.

The students were more involved in the group practice sessions. They talked to friends and reported their answers to the whole class. Students were asked to discuss in groups, but they were not allowed adequate time given the discussion questions required short answers and logical explanations. I could hear the students giving short answers but not providing explanations. During the lecture, the teacher kept encouraging students to ask questions about any points they could not understand. Interestingly, however, students rarely directed questions 
to the teacher. Those students at the back sometimes used their phones to look up words and take notes in their books.

In summary, Nathan was confident in his ability to teach his subject through English as he had strong content knowledge and teaching experience. He relied on the syllabus and used the teacher's books to modify the lessons. He felt worried about whether students would understand his explanations of business concepts understandably in English and helped first-year students to make sense of business terms by using simple language, giving practical examples or illustrating with graphs and charts. Nathan felt unable to engage students to interact in class activities because of the full curriculum and limited time. He wanted them to collaborate but did not build this into his lessons. As noted in the observation, students rarely responded to the teacher's questions. They participated in the practice sessions but mostly listened passively to the teacher's lectures.

\subsubsection{The use of English}

Nathan felt comfortable communicating in English as he used to study in an Englishspeaking country. He thought that he had mastered the content of this subject in English and that he could "use English to teach business concepts naturally without taking time to translate from Vietnamese into English".

Specifically, Nathan used a significant amount of English in class, noting that it was the requirement of the school to "get students familiar with the use of English in learning from the start". This requirement aimed to make students less dependent on the teacher's use of Vietnamese in class. Nathan was aware of students' difficulties in coming to terms with classes in English, stating that "using English completely would be tough for first-year students, so I had to encourage them to do further readings and recommend different materials to them". He realised from their questions that students had trouble understanding subject matter in English and sometimes explained concepts again in Vietnamese.

In his opinion, the language used in the textbook and slides was appropriate for first-year students. He did not spend much time preparing what he would say in the lessons. Instead, he reported:

Some business concepts cannot be exactly translated and understood in Vietnamese, so I had to use many examples to support them. I had to use spoken English or simple English in this case to make students understand them more easily.

He looked for relevant examples and found ways to express them in simple language for students to understand. 
Nathan felt that using English affected his teaching, noting that it was hard for him to express everything easily and flexibly in English. As a result, he felt his lectures to be somewhat boring and ineffective as students felt nervous and uninspired. Nathan realised that he should slow down the teaching pace to achieve the objectives of the lessons.

Nathan believed that students needed help as they had different English proficiencies. He viewed the English language as a barrier to learning subject matter and thought that the entry requirement of using written English test scores was not an appropriate way to assess communicative skills. Moreover, he felt that the English modules did not seem helpful to students and suggested that there should be tutors to help students with English problems.

Nathan did not set English objectives in his lectures (see Table 12) and prioritised academic subject content. His teaching approach of presenting objectives, lecturing on theory, and problem-solving practice was intended to give students an overview of content and to allow them to apply this in practice. Students were required to learn certain business concepts in academic English to deal with subject matter. Nathan conducted group work activities to encouraged students to interact with each other, but those activities appeared not to be effective.

In summary, Nathan had both teaching experience and confidence in using English as a medium of instruction. He believed that his use of English made the subject matter understandable for students. However, he had concerns about students' English proficiency and suggested that there should be a language support scheme for students. 
Table 12

Nathan's teaching activities compared to content-based English teaching principles (Gleeson, 2015).

\begin{tabular}{|c|c|c|c|c|c|}
\hline $\mathrm{P}_{\text {Activities }}^{\text {Principles }}$ & $\begin{array}{l}\text { Knowing how English } \\
\text { is learnt through } \\
\text { content }\end{array}$ & $\begin{array}{l}\text { Creating connections } \\
\text { between the students' } \\
\text { experiential } \\
\text { background and new } \\
\text { ideas }\end{array}$ & $\begin{array}{l}\text { Sustaining academic } \\
\text { rigour and } \\
\text { maintaining high } \\
\text { expectations }\end{array}$ & $\begin{array}{l}\text { Knowing the academic } \\
\text { language demands of } \\
\text { the subjects and } \\
\text { sustaining a focus on } \\
\text { academic language }\end{array}$ & $\begin{array}{l}\text { Engaging students in } \\
\text { quality interactions } \\
\text { that promote } \\
\text { productive and } \\
\text { receptive skills } \\
\end{array}$ \\
\hline $\begin{array}{l}\text { Using Q\&A activities to help students recall } \\
\text { key business concepts and key English } \\
\text { words. }\end{array}$ & & & $\checkmark$ & & \\
\hline $\begin{array}{l}\text { Introducing the objectives of the new } \\
\text { lessons. }\end{array}$ & & $\checkmark$ & $\checkmark$ & & \\
\hline $\begin{array}{l}\text { Lecturing new subject matters by explaining } \\
\text { the theory, teaching the concepts and } \\
\text { giving examples }\end{array}$ & & $\sqrt{ }$ & $\checkmark$ & & \\
\hline $\begin{array}{l}\text { Having students discuss in groups to find } \\
\text { out solutions to specific situations. }\end{array}$ & & $\checkmark$ & $\checkmark$ & & $\checkmark$ \\
\hline
\end{tabular}




\subsubsection{The use of digital technologies}

Nathan said that digital technologies provided him with support in his teaching (see Table 13). However, he did not report integrating many technologies into his lectures in class. He used a desktop computer and projector to show PowerPoint slides to students, using slides to include the objectives of each lesson, summarise the main points of lessons, and provide students with definitions of business concepts.

\section{Table 13}

Nathan's use of digital technologies to support his teaching

\begin{tabular}{|l|c|c|c|c|c|}
\hline \multicolumn{1}{|c|}{ Digital technology } & \multicolumn{2}{|c|}{ The Internet } & LMS & PowerPoint & $\begin{array}{c}\text { Computer } \\
\text { software }\end{array}$ \\
\hline & Websites & Google & & & \\
\hline Accessing information & $\checkmark$ & $\checkmark$ & & $\checkmark$ & \\
\hline Presenting information & & & & & $\checkmark$ \\
\hline $\begin{array}{l}\text { Processing or creating } \\
\text { digital objects }\end{array}$ & & & & & $\checkmark$ \\
\hline $\begin{array}{l}\text { Gaming or interactive } \\
\text { programmes }\end{array}$ & & & & & \\
\hline $\begin{array}{l}\text { Communicating or } \\
\text { collaborating }\end{array}$ & $\checkmark$ & & $\checkmark$ & & \\
\hline
\end{tabular}

Nathan had access to different open resources of reference through Google and the Internet. He downloaded slides by professors working in foreign universities, seeing these slides as appropriate for undergraduate students as the professors aimed to reach a general international audience. He reported adding relevant examples in Vietnamese context, or "updating the tables and charts in slides to illustrate the concepts".

Nathan spent time scanning charts from the main course books, drawing tables with updated statistics and using graphs to "help students memorise the concepts easily". He saw PowerPoint slides as useful ways to present the charts and graphs to students and believed that such visual aids would assist students to understand subject matter.

Using different web-based resources, he updated his content knowledge by finding a range of viewpoints related to his subject both online and offline. He reported that different interpretations of economic principles were not only written in different books or journals, but also discussed in many forums or academic groups on the Internet. Thus, he had to be aware of those viewpoints so that he could explain them to students whenever they asked probing questions. He also supported students' learning in different ways, including providing them with links to reference materials on websites in economics. 
Nathan said he wanted to integrate technologies into his assessment and thought that using technological devices would bring both teachers and students certain benefits. For example, students did their mid-term tests on computers. Nathan booked a computer room and scheduled time for students to sit the tests, and at the end students were asked to submit before receiving instant results. Nathan used a monitor computer to manage students' submissions and scores. He provided further detail in the following quote:

Doing a test on a computer helps to save time and money as printing costs. In my class, students advocated for sitting the computer-based tests as they could get their scores immediately. I did not have to mark their tests but selected questions in the test bank. However, the procedure was still complicated as I had to register to use the computer room and report to different administration staff.

Despite a few administrative constraints, Nathan and his students felt positively about digital assessment.

Nathan felt that the LMS at the university was not fully utilised. He used the LMS to contact students and give them further reading texts. He also uploaded the course syllabus to the LMS at the beginning of the semester so that students were aware of the objectives. Nathan communicated with students through the LSM but had to email them as well due to technical problems. Nathan was dissatisfied with the system:

It is not effective when the lecturer uses the LMS to upload lessons with the content that he cannot explain in class. It is also time-consuming to be involved in the students' group discussion online. Many lecturers are using LMS due to the policy of the university.

He explained that the university policy encouraged teachers to use the LMS by giving them bonus scores in their performance scoring scheme. From his perspective, he did not believe that students understood online lessons as clearly as the face to face mode. He felt that the system was not user-friendly, and that students found it inconvenient to use.

Nathan had his own website where he posted the content of his lessons including PowerPoint slides, further reading texts and study cases. Students were able to access the website to download study materials and Nathan mentioned that students often emailed him questions about the lessons. Not wanting to waste time answering the same questions in email form, he also encouraged students to interact with him directly:

I always encourage students to ask questions in class so that everyone can benefit from my answers. I arrange an office hour every Monday, Wednesday and Friday to meet students at my office for any consultancy. 
In summary, Nathan used digital technologies to assist his teaching. He did not integrate them much into his lectures in class because he thought that the nature of his subject and the time pressure did not allow for such integration. Nathan accessed online reference resources to update his content knowledge and offered students these resources. He advocated computerbased assessment for students and did not think that the LMS system at the university was effective or motivating.

\subsubsection{The students}

Six female students in the class participated in the focus group discussion. They expressed different levels of confidence in three areas of content knowledge, English competence and digital technology (see Table 14). All of them felt confident in using English for both communication and learning. However, they reported unfamiliarity with business terms in English. They admitted being concerned about understanding economics content, as they had never learnt it in high school and added that first-year students did not understand what knowledge and strategies were needed for learning. One student had experience in studying science subjects such as Mathematics, Biology, and Physics in English in high school. Three students said they used to selfstudy Mathematics in English at home to prepare for the Scholastic Aptitude Test (SAT) and American College Testing (ACT). The other two had not learnt any content subjects in English and both felt a lack of confidence as a result. Six students described themselves as being confident in using digital technologies such as office software (Word, Excel, and PowerPoint), electronic devices (laptops, computers, mobile phones, and projectors), applications (Dropbox, Google Drive, Podcasts, and Keynote) and social networks (Facebook, Twitter, and Instagram).

\section{Table 14}

A summary of students' profiles regarding their confidence from the most (1) to the least (3)

\begin{tabular}{|l|c|c|c|c|}
\hline \multirow{2}{*}{ Students } & \multirow{2}{*}{ Gender } & \multicolumn{3}{|c|}{ Field of confidence } \\
\cline { 3 - 5 } & & Content knowledge & English competence & Digital technology \\
\hline Student 1 & Female & 3 & 1 & 2 \\
\hline Student 2 & Female & 3 & 1 & 2 \\
\hline Student 3 & Female & 3 & 1 & 2 \\
\hline Student 4 & Female & 3 & 1 & 2 \\
\hline Student 5 & Female & 3 & 2 & 1 \\
\hline Student 6 & Female & 3 & 2 & 1 \\
\hline
\end{tabular}

All students wanted to learn economics through English. Four students felt motivated by the new learning context even though they found it challenging, believing they "would get used 
to it quickly as it was interesting". The other two who reported having confidence in English perceived some of the benefits of studying in English, as stated by Student 5:

Studying economics in English, I will have both sound content knowledge and good English competence to participate in any international environment. I can expand my vocabulary and practise the skill of thinking in English. I can save time and money to take some courses at a foreign language centre.

To achieve these objectives, the students thought they had to work hard, and they reported that they had to prepare for the lessons at home to be able to follow the lectures in class, as student 2 explained:

I have to read the textbook and slides at home. I look up new words and read the explanation to get a general understanding of concepts. However, I cannot handle exercises related to calculations. I need to do more exercises after class.

Added to the need for self-study, students complained that they could not concentrate on a full three-hour lecture. Their preparation only helped them stay focused and study effectively in the first half and they mentioned their difficulty following the teacher in the second half, as evidenced in this quote:

The teacher went through two chapters, so I felt tired and missed a lot of information. After the break, the teacher often focusses on exercises related to calculations and critical thinking, so I was not alert enough to understand them.

One student drew possible connections with their learning habits at high school where they usually had a five-minute break after each period of 45 minutes. At university, they had to study continuously for one and a half hours.

Students reported developing their own learning strategies for studying in English. Four students shared that they read books and prepared for the lessons in English. They only looked up Vietnamese meanings for key words. One student added that she sometimes "guessed the meanings of words in the sentences". The other two students used both Vietnamese and English textbooks, first reading the Vietnamese textbook to gain a general understanding of the key concepts. This helped them "understand and follow the lessons in English more easily". All students believed that memorising the business concepts entirely in English would be better for them and four of them agreed that listening to the teacher's lecture in English was an effective way to revise and memorise the terms in English. The other two students had concerns about this issue, suggesting the teacher should use both languages when necessary, as stated in the following quote: 
When my friend who studied this subject in Vietnamese asked me about a concept, I did not know its Vietnamese equivalent. I had to look it up in a dictionary, but I could not understand the explanation, and the Vietnamese equivalent in dictionary was not the same as what my friend had learnt.

This points to the fact that students were afraid of being confused and unable to understand the terms in their first language.

All students in the focus group thought that digital technologies were an important source of assistance for them. They reported that they were experienced in using technological equipment such as laptops, computers, tablets and mobile phones to support their study (see Table 15).

Students mentioned the provision of useful language support, with student 6 explaining that different dictionary applications available in tablets and smartphones were "user-friendly and convenient for learning business vocabulary in and after".

Students reported using Google as a search tool to find reference materials, noting they could "download a lot of electronic books related to their subjects from different websites". They accessed links that the teacher provided and visited his personal website for further reading texts, exercises, and study cases. Three students found online lectures provided by experts or professors from foreign universities, as evidenced in the quote below:

I searched on YouTube for videos of lectures that foreign professors posted. These lectures were in English. The professors only used simple language to explain business concepts. Some video clips using pictures to illustrate the examples were funny and easy to understand.

Another student used Facebook and got recommendations from senior students for videos of lectures. She had joined different Facebook groups in her school and at the wider university such as the Business Students group, Bell Club group and Mar-group. She added that she "also viewed Facebook pages of some lecturers where they often posted links to useful video clips related to economic subjects."

All students confirmed that they only used the LMS to see updated information about the course. Student 4 emphasised that "there were not enough inspiring activities to support the studying of subject matter as the page was boring and lacked interactions." 


\section{Table 15}

Students' use of digital technologies in the digital age learning matrix (Starkey, 2011)

\begin{tabular}{|c|c|c|c|c|c|c|}
\hline Aspects of learning & Doing & $\begin{array}{l}\text { Thinking about } \\
\text { connections }\end{array}$ & $\begin{array}{l}\text { Thinking about } \\
\text { concepts }\end{array}$ & $\begin{array}{l}\text { Critiquing and } \\
\text { evaluating }\end{array}$ & $\begin{array}{l}\text { Creating } \\
\text { knowledge }\end{array}$ & Sharing knowledge \\
\hline $\begin{array}{l}\text { Explanation of } \\
\text { aspects of learning: } \\
\text { Digital technology } \\
\text { use: }\end{array}$ & $\begin{array}{l}\text { Isolated } \\
\text { information. } \\
\text { Focus on } \\
\text { completing a } \\
\text { measurable task. }\end{array}$ & $\begin{array}{l}\text { Connecting } \\
\text { thinking. } \\
\text { Simple } \\
\text { connections made } \\
\text { within a context. } \\
\text { Compare and } \\
\text { share. }\end{array}$ & $\begin{array}{l}\text { Develop } \\
\text { conceptual } \\
\text { understanding of } \\
\text { 'big ideas'. }\end{array}$ & $\begin{array}{l}\text { Evaluating and } \\
\text { critiquing to } \\
\text { explore the } \\
\text { limitations and } \\
\text { potential of } \\
\text { information, } \\
\text { sources or } \\
\text { process. }\end{array}$ & $\begin{array}{l}\text { Creativity - } \\
\text { applying ideas, } \\
\text { processes and/or } \\
\text { experiences to } \\
\text { develop a new } \\
\text { reality. }\end{array}$ & $\begin{array}{l}\text { Sharing the new } \\
\text { knowledge through } \\
\text { authentic context } \\
\text { and gaining feedback } \\
\text { to measure value. }\end{array}$ \\
\hline $\begin{array}{l}\text { Accessing } \\
\text { information }\end{array}$ & \multicolumn{4}{|c|}{$\begin{array}{l}\text { Students used Google as a search tool to find reference materials. They had access } \\
\text { to links that the teacher provided and to his personal website for further reading } \\
\text { texts, exercises and study cases. They looked for online lectures provided by } \\
\text { experts or professors from foreign universities. }\end{array}$} & & \\
\hline $\begin{array}{l}\text { Gaming or } \\
\text { interactive } \\
\text { programmes }\end{array}$ & \multicolumn{3}{|c|}{$\begin{array}{l}\text { Students used mobile phone applications to look up the } \\
\text { meaning and definitions of business concepts and terms. }\end{array}$} & & & \\
\hline $\begin{array}{l}\text { Communicating or } \\
\text { collaborating }\end{array}$ & \multicolumn{6}{|c|}{$\begin{array}{l}\text { Students joined different student Facebook groups in their school or at the university such as Business Students group, Bell Club } \\
\text { group or Mar-group. They only used the LMS to obtain updated information about the course. }\end{array}$} \\
\hline
\end{tabular}


Students in the focus group also talked about their plans to improve their study techniques. They suggested taking notes carefully so they could revise the lessons at home, asking more questions in class to understand all concepts right away, or seeking more practical examples from the teacher or from different materials. Students also said they would like the teachers to provide them with more details about the test format and kinds of assessment as "some Facebook groups posting test papers used in previous years are not reliable" (Student 4).

All students insisted that group work was essential to their study, not only in class but also outside class. They thought that it was helpful when they could "help each other understand difficult points or do exercises". Yet, they were not satisfied with the group work activities in class, with Student 4 explaining the ineffectual nature of such activities, as she saw it: "The teacher gave us little time or simple questions that we did not feel motivated to discuss with each other". Moreover, it was difficult for first-year students to form groups, as they did not know each other. Some students confessed that they relied on Facebook to work with friends who they knew from high school and they sometimes communicated with friends studying at different universities to share materials or exchange information related to their subjects.

Some students were concerned about the choice of language in discussions as well as in interactions between the teacher and students in class, as Student 1 shared:

In group discussions, my friends often suggest using Vietnamese to quickly and easily exchange information, but we have to report our answers in English. We cannot have enough time to translate our answers into English, so we do not feel confident.

This may explain why there was so little interaction in class. Lack of time and having to switch between two languages restricted the students' ability to interact with each other or with the teacher.

In summary, students enjoyed studying economic in English. They considered it a challenge motivating them to try harder. They believed that they needed to prepare a lot at home to follow the lessons in class and relatedly, they reported struggling with so much subject content. Despite being confident in English, students found it difficult to understand and memorise business concepts and unfamiliar terms. Some thought that they needed to learn both English and Vietnamese meanings for the concepts. Students found digital technologies invaluable for both language support and sourcing reference materials and they tried to learn more about the subject from experts and professors through social networking. They collaborated and discussed with friends at different universities using Facebook, Messenger or mobile phones. Students believed that more group work would benefit them in the new learning context. 


\subsubsection{Summary}

Nathan had some experience in using English as a medium of instruction while his students were new to the context. Nathan used to study in an English-speaking country and used English to teach economic when he was a tutor. Meanwhile, his students were just starting their first year at university. They had graduated high school without any background knowledge in business. Participating in the High-Quality programme, they had to start studying economics through English in the first year. Although Nathan and his students were under pressure to cover the curriculum content, they all supported this EMI programme. They perceived it as an opportunity to improve their language proficiency and the students also expected to develop the ability to think in English and compete in international contexts. For this to happen, Nathan believed that a language support scheme for students was necessary.

Nathan had confidence in his strong content knowledge and ability to teach the subject through English. He expressed his confidence in the ability to manage the content and the syllabus of the subject. Although Nathan felt confident and competent in English, he reported finding it hard to express himself or give lectures effectively in the language. Being aware of the adversity that first-year students had to cope with, Nathan tried different strategies to help them but could not integrate technologies into teaching activities because of limited time.

The students were concerned that this EMI course was their first exposure to business concepts that seemed too hard for them to understand even in Vietnamese. Despite high levels of proficiency in written English, they had difficulty using English to learn economic subjects. To deal with this, they spent a great deal of time preparing for lessons at home and looking for more reference materials written in English and Vietnamese. They set themselves many exercises to understand the theory and felt worried about the unfamiliar test format and content. They reported using digital technologies frequently in their learning.

\subsection{Case 3 - Taylor}

The third case involved one teacher and four students in a nine-week EMI finance course. The teacher - Taylor - was first interviewed in the seventh week and the classroom observation and the student focus group were conducted in the eighth week. The teacher was interviewed again in the final week. This teacher had many years' experience of teaching finance through English; however, the students in her class had not studied finance in English before.

\subsubsection{The context}

The school where Taylor worked offered academic programmes at both undergraduate and post-graduate levels in finance and insurance. At the bachelor's level, this school ran both the 
High-Quality Programme and the Advanced Programme. In the High-Quality Programme, students started to study through English after completing two years of foundation subjects. Taylor had been involved in these two programmes from the beginning.

Sixty students studied in Taylor's High-Quality Programme class. They were third-year students starting to study financial subjects in their area of expertise. This class was wellresourced through the High-Quality programme. Students had access to the LMS managed by the teacher. They were allowed to use their own laptops and mobile phones in class for learning purposes.

\subsubsection{The teacher}

Taylor had been working as a lecturer at the university for more than 20 years. She had a bachelor's degree and a master's degree in economics and finance and had earned a full study scholarship for these degrees. As well as taking a one-month internship in an English-speaking country, she had worked as a teaching assistant for an international academic programme and as a freelance interpreter before starting her teaching career.

Taylor therefore had strong content knowledge in her subject due to her experience in teaching finance and working for financial companies. She was also involved in academic joint programmes shared between the university and its foreign counterparts in which she translated teaching and learning materials from English into Vietnamese. All these experiences provided her with confidence in her content knowledge.

Possessing many years' experience in using English as a medium to teach economics subjects, Taylor felt comfortable in using English because she had studied in an English-speaking country. Moreover, her experience as a teaching assistant and an interpreter in English helped her in teaching through English. Even so, she was slightly concerned about her English proficiency:

I was not formally trained in English. When I started to learn English, I did not have equipment such as computers, internet, tapes or cassette players nor the environment to practise. Although I can communicate in English, I still make a lot of mistakes in grammar and pronunciation.

She was pleased to be involved in the High-Quality Programme because the university offered benefits for participating teachers such as good working conditions and higher salaries. Besides, she was impressed with her students' attitudes:

Students in this class are good at critical thinking. They have "willing-to-learn" attitudes both in class and at home although their English competence is not good. As I frequently encourage them to try harder in their learning, they have become more motivated to improve themselves. 
Taylor specified her use of digital technology in class as limited and assumed it would take a long time to learn because of her ability. She reported that using technological equipment did not work well in her subject, as students could not follow the lessons, saying that she had to guide students to practise calculation step-by-step rather than showing them the results on screen. She mainly used digital devices such as mobile phones, laptops or desktop computers to access different sources of information online.

In summary, Taylor had considerable experience in teaching her subject in both Vietnamese and English. She felt confident in her strong content knowledge and she was comfortable with teaching through English although she worried about making mistakes. Satisfied with both the resources provided by the university and the positive attitudes of the students in the High-Quality Programme, she did not, however, feel confident about using digital technologies in class.

\subsubsection{Teaching content knowledge}

Her many years spent teaching the subject gave Taylor assurance in her content knowledge. As the course aimed to prepare students with basic knowledge in finance, she did not set too many content objectives for students. She was familiar with the syllabus that the school assigned for the course and thought that the set course objectives were appropriate for the level of the students. Taylor followed the syllabus closely when designing lessons, making "a detailed plan for each chapter" which included "the content focus of each lesson and identify[ing] which sections to present in class and which ones to assign for students' discussion or further reading".

Taylor emphasised the connections between the theories from her lessons and practice. She motivated students to engage in the lectures more actively by giving examples related to Vietnamese context and designing exercises enabling students to apply their understanding of key theories. However, she was aware that this could be a challenge for students who had limited English competence, so she tried to simplify the examples.

Her procedure for presenting business concepts to students was consistent. She first provided them with definitions of the concepts written in the course book. Then she explained the related theory and developed the formula for students to apply in practical exercises. She believed that this made it easier for students to comprehend the concepts.

Taylor applied different strategies to help students remember new concepts. She said that students were required to understand the theory and to memorise formulae to apply in exercises. She reported using tables to summarise main points and creating online quizzes or PowerPoint quizzes to consolidate what students had learnt at the end of each lesson: "I always make short 
questions or correct students' homework at the beginning of each lesson in order to review previous knowledge", she added.

Taylor was always thinking of ways to push students harder in their learning. She thought that giving students more exercises might be helpful in making them focus more on their learning. She recognised that "some students are still passive and distracted in their learning" and mentioned that she often questioned students during lessons to ensure focus and engagement, and to check understanding.

In the lecture I observed, Taylor conducted a range of teaching activities aimed to help students achieve set objectives (see Table 16).

Table 16

Classroom observation (Taylor): Summary of teaching steps

\begin{tabular}{|c|c|c|c|}
\hline No. & Teaching steps & Activities & Objectives \\
\hline 1 & Revision & $\begin{array}{l}\text { Homework correction: The teacher } \\
\text { summarised some key points from } \\
\text { the previous lesson and provided } \\
\text { students with answers of two } \\
\text { exercises assigned as homework. }\end{array}$ & $\begin{array}{l}\text { - To help students revise } \\
\text { previous knowledge and recall } \\
\text { business concepts and relevant } \\
\text { formulae. }\end{array}$ \\
\hline 2 & Lead-in & $\begin{array}{l}\text { Introduction: The teacher asked } \\
\text { questions connecting previously } \\
\text { learnt content to the new lesson (e.g. } \\
\text { current assets, short-term assets, } \\
\text { current liabilities, short-term debt), } \\
\text { then introduced key content of the } \\
\text { new lesson (e.g. short-term asset } \\
\text { management, short-term financing) } \\
\text { together with the objectives she } \\
\text { wanted students to achieve by the } \\
\text { end of the lesson. }\end{array}$ & $\begin{array}{l}\text { - To clearly inform students of } \\
\text { what they were going to learn. } \\
\text { so that they could focus on the } \\
\text { right points. } \\
\text {-To draw students' attention to } \\
\text { the relationships between } \\
\text { business concepts. }\end{array}$ \\
\hline 3 & $\begin{array}{l}\text { Presenting new } \\
\text { concepts }\end{array}$ & $\begin{array}{l}\text { Lecturing: The teacher used slides to } \\
\text { lecture on the theory, explaining the } \\
\text { concepts and giving examples. }\end{array}$ & $\begin{array}{l}\text { - To transmit the content to } \\
\text { students, having them } \\
\text { understand the economic } \\
\text { principles and how they are } \\
\text { implemented in real situations. }\end{array}$ \\
\hline 4 & Practice & $\begin{array}{l}\text { Exercises: The teacher asked students } \\
\text { to do some exercises in the course } \\
\text { book, then had students report their } \\
\text { answers which she wrote on the } \\
\text { blackboard. }\end{array}$ & $\begin{array}{l}\text { - To have students apply the } \\
\text { theory and use the formulae } \\
\text { they had learnt to solve practical } \\
\text { financial problems. }\end{array}$ \\
\hline
\end{tabular}




\begin{tabular}{|l|l|l|l|}
\hline 4 & Wrap-up & $\begin{array}{l}\text { The teacher corrected students' } \\
\text { answers and provided them with } \\
\text { further detailed explanations. } \\
\text { The teacher summarised the lesson's } \\
\text { key points and formulae in a table } \\
\text { and checked students' } \\
\text { comprehension by asking questions. }\end{array}$ & $\begin{array}{l}\text { - To consolidate what students } \\
\text { had learnt and ensure that they } \\
\text { could understand the theory and } \\
\text { apply it properly in practice. }\end{array}$ \\
& & & \\
\hline
\end{tabular}

The observation showed how Taylor used a desktop computer and projector to share the lesson content with students. She asked different types of questions to elicit students' previous knowledge, facilitate new content and check students' comprehension. I also observed how she used English to communicate with students. She provided students with Vietnamese equivalents of new business concepts and switched to using Vietnamese sometimes when explaining content knowledge and correcting exercises. Taylor said that she used Vietnamese to make challenging abstract business terms understandable for students. She believed that students needed to be clear about how to do the exercises, so using Vietnamese was beneficial for them. The black board was used frequently throughout the lecture and students were given hand-outs of slides and a copied course book. In terms of language use, students talked to each other in Vietnamese and used English when answering the teacher's questions (asked in English). Many students had their laptops and mobile phones on their desks and some of them used their laptop screens to view slides, take notes, and use online dictionaries. Students frequently worked together when assigned exercises. Some individuals asked the teacher questions in Vietnamese.

In summary, Taylor was confident with her knowledge of the subject. She used different strategies in her teaching activities such as giving meaningful examples, combining theory and practice, and using questions to engage students and consolidate their knowledge. She tried to find ways of encouraging students in their learning. Observations revealed both the teacher's and students' use of English and digital technologies in teaching and learning activities in class. While there was frequent language switching between English and Vietnamese, there was little integration of digital devices.

\subsubsection{The use of English}

Taylor told me that she enjoyed teaching her subject through English. She had started using English to teach economics more than ten years ago. She felt motivated to teach this way although it required her to invest more effort. She believed that she could teach her subject as comfortably in English as in Vietnamese when students were good at English. However, in this 
class, she thought that the students' low level of English proficiency was an obstacle for her. She explained:

I have to use Vietnamese quite often when summarising lessons, answering students' questions or correcting exercises as students' English is not good. They can read the texts in their book to do exercises, but they are limited in listening comprehension in English.

Taylor thought that this affected the interaction between her and the students in class. She felt demotivated sometimes when students would not speak in class and found it hard to know how much students were able to understand, noting that "students do not answer my questions, nor do they engage in discussion due to their fear of losing face or shyness".

Taylor used the language written in the course book when giving students definitions of business concepts. She believed that this would help students follow the lessons easily. She reported that although she did not simplify the content or objectives of the lessons, she found switching from English to Vietnamese was helpful at times:

When adding extra explanations to clarify the concepts, I do not make these complicated. I provide them with basic examples. I have to speak slowly or use Vietnamese to make them understand. Sometimes I skip some examples that do not influence students' comprehension.

This shows that she adjusted her instruction to fit students' levels of English.

Taylor believed that students' English improved in the process of learning content during the course. She did not set any objectives for English learning as in her opinion English competence was enhanced through learning the modules in English. Besides, she said that students attending the High-Quality programme had sat a placement test in English as a requirement. Thus, she thought that they had adequate English proficiency for this course. However, she suggested that the school and the university should consider setting certain English requirements for students taking EMI courses in economics, given the specific requirements

Taylor did not set English objectives in her lectures (see Table 17). Rather, she created learning activities in which students were expected to develop their understanding of subject matter and academic English through doing calculation exercises, listening to lectures on theory and discussing questions. Apart from question and answer sessions, students in Taylor's class appeared not to engage much in interactions that had the potential to improve their language skills. 
Table 17

Taylor's teaching activities compared to content-based English teaching principles (Gleeson, 2015).

\begin{tabular}{|c|c|c|c|c|c|}
\hline $\mathrm{C}_{\text {Activities }}^{\text {Principles }}$ & $\begin{array}{l}\text { Knowing how English } \\
\text { is learnt through } \\
\text { content }\end{array}$ & $\begin{array}{l}\text { Creating connections } \\
\text { between the students' } \\
\text { experiential } \\
\text { background and new } \\
\text { ideas }\end{array}$ & $\begin{array}{l}\text { Sustaining academic } \\
\text { rigour and } \\
\text { maintaining high } \\
\text { expectations }\end{array}$ & $\begin{array}{l}\text { Knowing the academic } \\
\text { language demands of } \\
\text { the subjects and } \\
\text { sustaining a focus on } \\
\text { academic language }\end{array}$ & $\begin{array}{l}\text { Engaging students in } \\
\text { quality interactions } \\
\text { that promote } \\
\text { productive and } \\
\text { receptive skills }\end{array}$ \\
\hline $\begin{array}{l}\text { Summarising key points in the previous } \\
\text { lesson and correcting students' homework. }\end{array}$ & & $\checkmark$ & $\checkmark$ & & \\
\hline $\begin{array}{l}\text { Asking questions related to prior learning } \\
\text { that related to the new lesson. }\end{array}$ & & $\checkmark$ & $\checkmark$ & & $\checkmark$ \\
\hline $\begin{array}{l}\text { Introducing key content of the new lesson } \\
\text { together with the objectives she wanted } \\
\text { students to achieve at the end of the lesson. }\end{array}$ & & & $\checkmark$ & & \\
\hline $\begin{array}{l}\text { Lecturing new subject matter by explaining } \\
\text { the theory, teaching the concepts and } \\
\text { giving examples. }\end{array}$ & & $\checkmark$ & $\checkmark$ & & \\
\hline $\begin{array}{l}\text { Getting students to practise and correcting } \\
\text { their answers. }\end{array}$ & & $\sqrt{ }$ & $\sqrt{ }$ & & \\
\hline
\end{tabular}


In summary, Taylor was comfortable with teaching her subject through English as she had many years' experience in the job as well as a clear preference for EMI. However, she did not appear to understand that EMI instruction required specific teaching approaches. She reported using Vietnamese in class to compensate for the students' low level of English proficiency but did not create opportunities for interaction between the teacher and students. Taylor believed that her students' understanding of business concepts in English improved naturally although objectives for English learning were not set. She thought that entry to this EMI programme should be restricted to students whose English proficiency was at a level that would allow them to study economics through English.

\subsubsection{The use of digital technologies}

Taylor said that she was not confident about using technological equipment or tools in her teaching. She said that she only used technologies for basic purposes such as accessing or presenting information, contacting students and creating quizzes (see Table 18).

\section{Table 18}

Taylor's use of digital technologies to support her teaching

\begin{tabular}{|l|c|c|c|c|c|}
\hline \multicolumn{1}{|c|}{$\begin{array}{c}\text { Digital } \\
\text { technology }\end{array}$} & \multicolumn{3}{|c|}{ The Internet } & LMS & PowerPoint \\
\hline & Websites & Google & YouTube & & \\
\hline $\begin{array}{l}\text { Accessing } \\
\text { information }\end{array}$ & $\checkmark$ & $\checkmark$ & $\checkmark$ & & \\
\hline $\begin{array}{l}\text { Presenting } \\
\text { information }\end{array}$ & & & $\checkmark$ & & $\checkmark$ \\
\hline $\begin{array}{l}\text { Processing or } \\
\text { creating digital } \\
\text { objects }\end{array}$ & & & & $\checkmark$ & $\checkmark$ \\
\hline $\begin{array}{l}\text { Communicating } \\
\text { or collaborating }\end{array}$ & & & & $\checkmark$ & \\
\hline
\end{tabular}

Taylor commonly used power point slides to present in class. With financial equations, however, she used a traditional black board as she believed this helped students understand the process and how to apply formulae, as explained in this quote:

I summarise key points using slides which were given to students at the beginning of the course and uploaded to the LMS. I do not use slides when correcting exercises for students as students cannot follow calculating procedure. If I did, they would copy the answers only.

She was afraid that students would passively copy the answers if she used slides. Moreover, it would take her time to design those slides.

Taylor mostly used digital technologies to source information and supplementary materials, using "the Internet for many hours every day to search for relevant content 
knowledge". She presented that she frequently used her mobile phone and laptop to surf financial websites or financial journals to look for real life examples that would support her lessons. She believed that Internet-based websites were a good resource for her teaching job. She reported:

I download video clips from YouTube to show students in class. I use those clips to conduct students' discussion with guiding and comprehension questions. I also use video cases included in the course book package that I get from a school at the university.

She found that her students were especially engaged with video cases.

Taylor integrated the LMS into her teaching. She created quizzes using PowerPoint or Moodle on the LMS to help students review previous lessons at the beginning of each lecture. Quizzes were uploaded on the LMS, as described in the following quote:

Creating quizzes on the LMS took me some time, but I was collaborating with another teacher who shared my class. The I-spring application helps to mark these quizzes and provide students with the results as soon as they complete the quizzes. I sometimes print some quizzes and got students to do them in class. I can check students' attendance and use the results for students' mid-term tests.

She said that she also integrated digital technologies into some teaching activities in class. She gave the following example:

I let students use the Internet to search for the mission and vision of a real company so that they could understand how companies set objectives to comply with their financial situation. Students are required to look for the price of some companies' stock in the lesson about financial pricing.

These activities involved comparing information in practice and content learnt in class by using the Internet to access practical examples. She allowed students to use their mobile phones and laptops in class, but she thought they sometimes used those devices ineffectively. She encouraged her students "to use their phones or laptops to look up words when necessary". She found that many students took photos of her slides rather than taking notes, and she was not sure "if they have reviewed lessons with those photos".

In summary, Taylor had little confidence in using digital technology. She commonly used technological devices to present information and access reference sources. She integrated the LMS and the Internet into teaching activities and let students use digital devices in class to support their learning but doubted the effectiveness of this practice.

\subsubsection{The students}

Four students participated in a focus group - one male and three females. They expressed different levels of confidence in three areas of content knowledge, English competence and digital technology (see Table 19). One student felt confident in his English proficiency. Two students 
were less confident, but able to use English in studying the subject. One student reported having a low level of English proficiency. All students thought that they had sound background knowledge to understand the content of the subject. They said that they were familiar with using different digital technologies in their study such as office software (Word, PowerPoint, and Excel), electronic devices (laptops, mobile phones, and projectors), applications (Dropbox, Google Drive, and Podcasts) and social networks (Facebook, and LinkedIn). Student 1 explained:

Digital technologies have become a crucial part of my study. I have a habit of using them to access information in different fields of interest. I am used to exploring online knowledge through English. This offers me a wide range of resources and great opportunity to enhance both English proficiency and content knowledge.

\section{Table 19}

A summary of students' profiles regarding their confidence from the most (1) to the least (3)

\begin{tabular}{|l|c|c|c|c|}
\hline \multirow{2}{*}{ Students } & \multirow{2}{*}{ Gender } & \multicolumn{3}{|c|}{ Field of confidence } \\
\cline { 3 - 5 } & & Content knowledge & English competence & Digital technology \\
\hline Student 1 & Male & 3 & 1 & 2 \\
\hline Student 2 & Female & 3 & 2 & 1 \\
\hline Student 3 & Female & 3 & 2 & 1 \\
\hline Student 4 & Female & 1 & 3 & 2 \\
\hline
\end{tabular}

Four students reported having little experience in studying a subject through English. Two students said that they had found online courses in economics taught through English by themselves, as evidenced by Student 1: "Last year, I studied microeconomics and macroeconomics in English by going online, looking for materials, and watching lectures on YouTube". He believed that this experience would prepare him to study financial subjects through English at university.

All four students wanted to learn their subjects through English. The student claiming a high level of English proficiency felt happy about using English for both learning and general purposes. Even the student who had the least confidence in English preferred this medium of instruction. She said, "I like studying this subject in English as it has required me to stay more focused and invest more time. That pushes me to try harder in my study."

Student participants mentioned different challenges of studying in English. The student who was competent in English reported struggling with content knowledge, as he was not good at subjects requiring figures and calculation. He said that he did not have trouble understanding English as the teacher also spoke slowly and repeated her explanations two or three times. In contrast, the student with lower English proficiency found the language was a barrier to learning, 
especially when her financial vocabulary was limited. The two other students shared the same problem:

When I listen to a discussion on the subject in Vietnamese, I can understand the content. However, when it is discussed in English, I cannot get the point, but try to guess from some key words and remember formulae to do exercises.

This suggests that students found it hard to understand new content in English.

The students prepared to study through English in different ways. In addition to reading the course book at home, they bought materials written in Vietnamese to become familiar with terms in the field of finance. They said that this helped them not only understand the content but also to participate more easily in discussions with their friends who studied the same subject in Vietnamese. Although these students believed that they were getting used to listening to English, they thought that the teacher's use of both languages was helpful for them. One student said that she needed to learn concepts in Vietnamese, then translate them into English to memorise the content. The other three students reported using English, as evidenced by Student 2 below:

I look up meanings of some key words to understand business concepts, then try to memorise formula by doing exercises. I do these steps mainly through English as I find it difficult to understand definitions of concepts translated to Vietnamese.

All students confirmed that they worked to apply formulae to solve financial problems. They said that they did as many exercises as possible to memorise formulae even though they sometimes did not understand the concepts clearly.

The students worried about achieving content objectives in the course. They were afraid that their English language proficiency prevented them from fully understanding the business concepts and theory. They said that they depended on the teacher who decided what to focus on in the subject. Student 3 said:

I am afraid that the teacher has to simplify or skip some tough content or explanations. She does not give us many examples of real situations but focuses on the course book. She has to repeat key points several times, so she does not have sufficient time for other parts.

The students were afraid that their English proficiency prevented the teacher from covering all course content.

Students said that they used digital technologies such as mobile phones and laptops frequently to support their learning (see Table 20). All students stated that Google search and YouTube were their favourite websites. They accessed Google to find materials as well as links to websites providing them with relevant subject matter and they reported subscribing to YouTube to access video clips of lectures given by professors or experts in finance from international 
universities or educational institutions. One student felt that it was sometimes difficult for her to understand those clips in English.

The four participants also believed that mobile phones were one of the most helpful devices supporting their study. They mainly used their phones to look up meanings of key words. Student 4 added that she took photos of the teacher's exercise corrections on the board and audio-record the lectures.

Students also reported participating in online groups to get help in their learning. A student who frequently used English online said that he preferred discussing issues in online forums as his Vietnamese classmates did not want to speak English. He listened to podcasts to improve his English and joined financial and marketing groups on the Internet. The three other students described spending time on social networks, as evidenced by Student 1 :

I am in a group of students majoring in finance on Facebook. Many members share links to useful materials related to this subject. They also raise some topics for everyone to discuss and exchange information. I can access more practice exercises and answer keys there.

This indicates that students found online social interaction important for their learning.

Students were uninterested in the LMS. They said that both the content and interface of the LMS were boring. They only accessed the LMS to remain updated on the teacher's announcements or to download and upload homework. They complained that the teacher posted "slides in pdf format instead of SCOM (System Centre Operations Managers) which could include interactive quizzes". Students suggested that the teacher should post videos of lectures or summary clips to help them review the lessons at home.

Students perceived that their English proficiency was improving. They said that they were expanding the range of vocabulary, especially in the field of finance. This helped them to understand subject matter discussions and they were able to use financial terms to present opinions. They thought that learning finance in English offered them exposure to business English at a high level because they had access to reading materials, online information, and financial groups. 


\section{Table 20}

Students' use of digital technologies in the digital age learning matrix (Starkey, 2011)

\begin{tabular}{|c|c|c|c|c|c|c|}
\hline Aspects of learning & Doing & $\begin{array}{l}\text { Thinking about } \\
\text { connections }\end{array}$ & $\begin{array}{l}\text { Thinking about } \\
\text { concepts }\end{array}$ & $\begin{array}{l}\text { Critiquing and } \\
\text { evaluating }\end{array}$ & $\begin{array}{l}\text { Creating } \\
\text { knowledge }\end{array}$ & Sharing knowledge \\
\hline $\begin{array}{l}\text { Explanation of } \\
\text { aspects of learning: } \\
\text { Digital technology } \\
\text { use: }\end{array}$ & $\begin{array}{l}\text { Isolated } \\
\text { information. } \\
\text { Focusing on } \\
\text { completing a } \\
\text { measurable task. }\end{array}$ & $\begin{array}{l}\text { Connecting } \\
\text { thinking. } \\
\text { Simple } \\
\text { connections made } \\
\text { within a context. } \\
\text { Compare and } \\
\text { share. }\end{array}$ & $\begin{array}{l}\text { Developing } \\
\text { conceptual } \\
\text { understanding of } \\
\text { 'big ideas' }\end{array}$ & $\begin{array}{l}\text { Evaluating and } \\
\text { critiquing to } \\
\text { explore the } \\
\text { limitations and } \\
\text { potential of } \\
\text { information, } \\
\text { sources or } \\
\text { process. }\end{array}$ & $\begin{array}{l}\text { Creativity - } \\
\text { applying ideas, } \\
\text { processes and/or } \\
\text { experiences to } \\
\text { develop a new } \\
\text { reality. }\end{array}$ & $\begin{array}{l}\text { Sharing the new } \\
\text { knowledge through } \\
\text { authentic contexts } \\
\text { and gaining feedback } \\
\text { to measure value. }\end{array}$ \\
\hline $\begin{array}{l}\text { Accessing } \\
\text { information }\end{array}$ & \multicolumn{4}{|c|}{$\begin{array}{l}\text { Students accessed Google to source materials as well as to find links to websites } \\
\text { providing them with relevant subject matter. They subscribed to YouTube for video } \\
\text { clips of lectures given by professors or experts in finance from international } \\
\text { educational institutions. }\end{array}$} & & \\
\hline $\begin{array}{l}\text { Gaming or } \\
\text { interactive } \\
\text { programmes }\end{array}$ & \multicolumn{4}{|c|}{ Students did quizzes posted on the LMS as suggested by the teacher. } & & \\
\hline $\begin{array}{l}\text { Communicating or } \\
\text { collaborating }\end{array}$ & \multicolumn{6}{|c|}{$\begin{array}{l}\text { Students participated in online groups to get help in their learning. They used online forums or social networks. They accessed } \\
\text { the LMS to stay up to date on the teacher's announcements or to download and upload homework. }\end{array}$} \\
\hline
\end{tabular}


All students thought that the teacher's teaching methods worked for them. They said that the teacher spoke slowly enough for them to understand the lessons, summarised concepts logically by combining theory and formulae, consolidated subject matter frequently by asking questions, and knew when to use Vietnamese to ensure that they understood the lessons. Student 2 summarised: "The teacher creates a positive atmosphere in class by discussing with us and giving us thorough guidance with her sense of humour". However, students also reported some limitations that the teacher could address. Student 1 explained:

The teacher's pronunciation and intonation are sometimes difficult for us to understand. She uses Vinlish (Vietnamese English) to make it understandable for everyone, but I have to guess what she really means. I feel bored sometimes when the lecture is slow, and the teacher repeats a point many times.

Three students with good English worried that the teacher' incorrect pronunciation would affect their English proficiency.

These students believed that they needed to improve their English to study the subject better. One student said that she was taking an English course at a language centre. Two others reported trying hard to learn English at home but felt disappointed with the modules of English they were taking, seeing them as ineffective because there were students at different levels in one class. The English teachers had to teach slowly, and the lessons were simple and boring. One student suggested that there should be a special English for finance module to prepare students for the programme.

In summary, none of the participating students had learnt the subject through English but found it motivating to accept this challenge. Students reported different difficulties such as understanding the content or the English language. They accessed materials in both languages to better understand the lessons and they used digital devices such as laptops and mobile phones to access the LMS or other reference resources, and installed applications to look up business terms. They also joined online communities such as online forums or Facebook groups to exchange information. Students felt motivated by the teacher's teaching strategies but were concerned about her English pronunciation. They believed that their English vocabulary for finance was improving and they felt responsible for developing their own English proficiency.

\subsubsection{Summary}

Taylor had a lot of experience in teaching her subject through English. She had strong content knowledge, and she felt this translated into teaching the subject well. Despite this confidence in using English in class, Taylor said that she still made mistakes in grammar and pronunciation. She tried to use as much English as possible but chose to translate into Vietnamese 
when correcting students' exercises on the board and explaining abstract business concepts. She found that her interactions with students in class were limited and attributed this to her students' limited English proficiency. Taylor did not use digital technologies much in her teaching as she said that her literacy in technology was limited.

The case study students were new to the learning context of EMI and had varying levels of English proficiency. They reported some concerns about learning subject matter through another language and struggled to understand lessons in English at the beginning of the course. They believed that they had now become used to listening to English and appreciated the teacher's use of two languages in class so that they could not miss any significant information. They said they were familiar with using digital technologies in their learning.

Both the teacher and students felt motivated in participating in the new teaching and learning context. Taylor felt that she benefited from the good working conditions and higher payment to EMI teachers offered by the university. She also expressed satisfaction with the learning attitudes of students in the programme even though their limited English proficiency sometimes affected her motivation. Student participants believed that the programme was a challenge that motivated them to stay focused, study outside class, and try their best.

\subsection{Case 4 - Nancy}

The fourth case involves a teacher and seven students in a nine-week EMI commerce course. The teacher participated in the first interview in the seventh week and in the second interview two weeks later. The classroom observation was conducted in the eighth week, and then seven students joined in a focus group in the same week. Students had not previously studied any subjects in English while this was the second course the teacher had taught in English.

\subsubsection{The context}

The school where Nancy worked offered undergraduate and graduate programmes in business information systems and e-commerce. The school was designing syllabi for the academic content of the Advanced Programme for the teaching and learning of core subjects in English. The courses that Nancy was responsible for were considered pilots for the programme.

The case study class included 43 students majoring in e-commerce. They were third-year students starting to specialise in specific areas of economics. All students had agreed to participate in the pilot course where they studied commerce through English, and at the same time, these students were taking their fourth Business English module taught by teachers of English from the School of Foreign Languages for economics specifically. This was the last English 
module at pre-intermediate level integrating communicative skills in business English based on the course book Market Leader.

Nancy conducted the course in the computer room where each student was equipped with a desktop computer. Nancy used the teacher's monitor to access her students' computers and set learning activities. Students could also use their own laptops and mobile phones for learning purposes in class. In addition, other facilities such as screens, projectors and sound systems were available in the room. Nancy required students to access some of the course content through LMS.

\subsubsection{The teacher}

Nancy had been working as a lecturer at the university for nearly eight years. She had a bachelor's degree in business information technology and had earned a full scholarship to study a master's degree in an English-speaking country. She had some experience working in business before starting her teaching career.

Nancy felt confident in her ability to use English both in daily life and at work. She felt that having lived and studied in an English-speaking country she had achieved a good level of English competence, especially for communicative purposes. She had two years' experience of teaching through English. She had participated in an international teaching programme as a tutor for students majoring in different areas of economics. In this programme, she had mainly used English to support students with their subject matter and assignments. Then, she proposed to the university authority that she should pilot teaching her subject in English. She believed that her English proficiency was improving through the practice of teaching.

Nancy said that using English as a medium of instruction had done wonders for her teaching career, as stated in this quote:

This is a valuable experience that I can add to my CV, which will help me build my own brand. I can achieve growing recognition among limited teaching staff who can teach economics subjects in English. Apart from that, I can get good payment when using English to teach my subject.

She perceived that participating in EMI programmes was an opportunity to further her career.

Nancy had strong content knowledge in her subject. From hands-on experience of working and teaching, she had developed sound digital knowledge and demonstrated skills in using technology. Having studied information technology, she said that she was familiar with an abundance of technological tools and devices applied in business information systems or electronic commerce. She said, "My subject involves using electronic equipment in commerce, so 
I need to introduce to students relevant tools in practice. Due to the availability of searching tools, it is not difficult to integrate advanced technology into classroom activities".

Nancy's teaching philosophy was to create a comfortable learning environment for students to cultivate their knowledge. She tried to establish a friendly relationship with her students so that they could feel free to express themselves and become inspired to contribute to the lectures.

In summary, Nancy was highly motivated to teach her subject using English as a medium of instruction. She wanted to establish her own professional image and become recognised as a lecturer who can teach economics in English. As a specialist in the field of information technology, Nancy was self-assured in her subject content and ability to use digital technology skilfully in class.

\subsubsection{Teaching content knowledge}

Nancy believed that the teacher needed to share content objectives for each lesson and said that setting lesson objectives helped her design appropriate activities for students. She reported that she easily managed the course content as she was responsible for designing and revising the subject syllabus in the school. She explained that she was assigned by the school to "review the subject syllabus to modify the objectives and the content of the course, edit slides and update them with latest examples or study cases". This was formal recognition of her content knowledge.

Nancy had limited experience in teaching her subject through English and expressed concern about her teaching methods:

In the previous course, I divided students into groups and had them to prepare presentations. I would give feedback and summarise key points. I wanted students to work hard by reading the textbook and preparing for the lessons at home. However, it didn't seem to be effective.

Nancy observed that many students did not work hard in her subject and attributed this to their lack of autonomy to work outside class. She said that they only focussed on their lectures. She did not have enough time to fully explain subject matter in class, which seemed to result in limited content learning. Thus, she "decided not to use student's presentation activities but spend time on lecturing and discussing between the teacher and students".

Nancy said that her subject involved a wide range of business concepts and procedures in which technology was used. Therefore, she wanted students to understand and apply their learning in practice. She reported using varied strategies to facilitate learning and elicit information from students: 
I often start with study questions to inform students of the lesson objectives. I check their understanding of the lesson by getting them to answer those questions at the end. With business concepts or definitions, I usually conduct brainstorming or discussing activities so that students can use their background knowledge to express their opinions.

Nancy believed that eliciting knowledge from students would help them actively contribute to the lesson. She would give feedback and develop definitions with the students, seeing this as a way to help students to memorise business concepts better and become more motivated in class.

Nancy helped students explore business procedures by providing them with clear instructions about how to use electronic tools. She reported designing specific tasks for students to apply electronic tools in business procedures:

I showed a video clip about how Business Intelligence (BI) operated, and then asked them to go online using $B I$ to search for a definition or any information about it. The students had some time to read and summarise information, then report to the whole class.

In this way, she encouraged students to interact and offer feedback to peers before she gave them a final evaluation.

Nancy also found that she sometimes had to simplify the content and focus on foundation knowledge. Wanting to make sure that students achieved the core objectives of the course, she frequently rephrased definitions of business concepts in the textbook using simpler language that students could understand.

Nancy insisted that lectures must include practical applications, as is evidenced in the following quote:

I had to look for updated news or examples to show the students real business contexts. These examples are usually common among students and part of their interests, so they are attracted and motivated. For example, to illustrate Big Data, I got students to think about social networks like Facebook and the Presidential election in the United States.

Accessible of content is therefore a clear concern for Nancy.

In the lecture I observed, Nancy conducted a range of teaching activities aimed to help students achieve the content objectives (see Table 21).

Table 21

Classroom observation (Nancy): Summary of teaching steps

\begin{tabular}{|l|l|l|l|}
\hline No. & Teaching steps & Activities & Objectives \\
\hline 1 & Revision & $\begin{array}{l}\text { The teacher summarised key points } \\
\text { from the previous lesson. }\end{array}$ & $\begin{array}{l}\text { - To have students recall the } \\
\text { content of the previous lessons. }\end{array}$ \\
\hline
\end{tabular}




\begin{tabular}{|c|c|c|c|}
\hline & & $\begin{array}{l}\text { The teacher checked if students had } \\
\text { any problems doing their individual } \\
\text { tests online. } \\
\text { The teacher responded to a student's } \\
\text { question by setting a group-work } \\
\text { discussion. Students were asked to } \\
\text { access an article saved on their } \\
\text { computers, read the text and discuss } \\
\text { set questions. }\end{array}$ & \\
\hline 2 & Lead-in & $\begin{array}{l}\text { Introduction: The teacher introduced } \\
\text { the lesson and provided students with } \\
\text { some study questions. } \\
\text { For example: } \\
\text { - Why do organisations need to } \\
\text { manage their business } \\
\text { process? } \\
\text { - What are the stages of } \\
\text { business process } \\
\text { management? }\end{array}$ & $\begin{array}{l}\text { - To direct students towards the } \\
\text { lesson objectives by prompting } \\
\text { them to think about the answers } \\
\text { to given questions. }\end{array}$ \\
\hline 3 & $\begin{array}{l}\text { Presenting new } \\
\text { concepts }\end{array}$ & $\begin{array}{l}\text { Lecturing: The teacher used questions } \\
\text { to elicit information from students } \\
\text { and explained the key concepts and } \\
\text { processes summarised in slides. The } \\
\text { teacher included charts, graphs and } \\
\text { examples in slides to clarify the } \\
\text { definitions of concepts. }\end{array}$ & $\begin{array}{l}\text { - To teach students business } \\
\text { concepts by having students } \\
\text { explore the practical examples } \\
\text { and business study cases so that } \\
\text { they could understand the } \\
\text { concepts and processes. }\end{array}$ \\
\hline 4 & Discussion & $\begin{array}{l}\text { The teacher used three video clips to } \\
\text { facilitate students' discussion on the } \\
\text { business concepts and procedures. } \\
\text { The first two clips aimed to provide } \\
\text { students with information about the } \\
\text { business content. Students were } \\
\text { asked to watch and answer some } \\
\text { questions about the content in the } \\
\text { clips. The third clip was a real case of } \\
\text { a company. Students were given } \\
\text { some questions to discuss in groups } \\
\text { after watching the clip three times. }\end{array}$ & $\begin{array}{l}\text { - To encourage students to work } \\
\text { together to explore the subject } \\
\text { matter. } \\
\text { - To stimulate students' } \\
\text { opinions. }\end{array}$ \\
\hline 4 & Wrap-up & $\begin{array}{l}\text { The teacher asked students to report } \\
\text { their group's answers, and then to } \\
\text { offer feedback to each other. } \\
\text { The teacher summarised the key } \\
\text { content included in the case study. }\end{array}$ & $\begin{array}{l}\text { - To consolidate what students } \\
\text { had learnt and make sure that } \\
\text { they could understand the } \\
\text { concepts through real practice } \\
\text { with business case studies. }\end{array}$ \\
\hline
\end{tabular}


I observed several teaching and learning activities conducted in class. The teacher used both English and Vietnamese to lecture. She used English a great deal but translated into Vietnamese at the end of each point. She showed slides to students using a desktop computer and projector. Lesson slides included summarised key content, and used charts, graphs and video clips to assist the learning. The teacher also engaged the students in the learning using questions and answers, group work discussion and problem-solving case studies. The students used the desktop computers to follow the lecture by accessing a specific drive where the teacher shared the lesson slides and e-books. Many students used their mobile phones in class while some students took notes in their notebooks. Students spoke Vietnamese in group discussions and when answering the teacher's questions.

In summary, Nancy had confidence in her strong content knowledge. She was in charge of designing the course syllabus, so she was clearly aware of the objectives set for students. Despite this, she felt concerned about her teaching methodology due to her lack of experience in teaching through English. She employed a range of strategies to facilitate students' learning and tried to provide students with practical applications but reported having to simplify the content for ease of understanding.

\subsubsection{The use of English}

Nancy believed that using English to teach her subject would be beneficial for both the teacher and her students. She felt it was a good opportunity for her to practise her English skills frequently and believed that she made it easier for students to study the subject in English:

In the subject, students work with a textbook written in English. I am also using slides in English when lecturing, so I think that it is better for students to study in English so that they can not only know technical terms but also communicate in English.

Nancy was sure that students could easily follow the content of her lessons but was uncertain if students could understand the whole lessons in English. She said that she talked to students after the first few lessons in order to make improvements to enhance learning. She reported, "Many students told me that they could not understand the lessons totally taught in English. They suggested that I should use Vietnamese to explain important points". This explains why she used both English and Vietnamese in class although she tried to speak as much English as possible.

Nancy thought that it was a challenge to teach in English. She said that she had had difficulties in her first year as she had to prepare every word she wanted to say in class. She used to feel stressed when the programme required her to tutor students entirely in English. However, 
she was growing in confidence in this area. She felt she had gained hands-on experience from the course and knew how to use English in class. She said, "Whenever I find that students are confused, I will switch into Vietnamese to make them understand better". This limited the pressure of using English all the time.

Nancy felt that she benefited from participating in the EMI training course. She thought that the course provided a range of techniques, which was good for teachers who had not had opportunities to study abroad. She reported selecting some techniques such as guiding questions, and choice of language to clarify or check comprehension, which were easy for her to apply in class. She perceived it useful for her "usage of English as a classroom language, which is sometimes different from language for daily communication". Moreover, when explaining business terms, Nancy found that she needed to paraphrase the definitions written in the textbook and rewrite them in simpler language so that students could understand. Nevertheless, Nancy said that she tried to expose students to as much English as possible, and to encourage them to interact using English in class:

All materials that I provide students are in English. I encourage students to use as much English as they can in discussions, answering questions, assignments or tests. I inform students that they will get bonus marks for using English in their assignments.

Nancy did not set objectives for English teaching in class but wanted to help students improve their English through her teaching activities (see Table 22) which were designed around the content objectives. She first focused on the academic content knowledge required for students to complete the course. To do that, she used the academic English that the students needed to understand the subject matter. Her use of simple questions and selection of video clips in English were designed to help students learn content but also English. She wanted students to become familiar with a learning environment where English was used as an instruction medium, and as such conducted frequent discussion activities to promote students' interactions, aiming to firstly improve receptive skills of reading and listening, then productive skills of writing and speaking. 


\section{Table 22}

Nancy's teaching activities compared to content-based English teaching principles (Gleeson, 2015).

\begin{tabular}{|c|c|c|c|c|c|}
\hline${ }_{\text {Activities }}^{\text {Principles }}$ & $\begin{array}{l}\text { Knowing how English } \\
\text { is learnt through } \\
\text { content }\end{array}$ & $\begin{array}{l}\text { Creating connections } \\
\text { between the students' } \\
\text { experiential } \\
\text { background and new } \\
\text { ideas }\end{array}$ & $\begin{array}{l}\text { Sustaining academic } \\
\text { rigour and } \\
\text { maintaining high } \\
\text { expectations }\end{array}$ & $\begin{array}{l}\text { Knowing the academic } \\
\text { language demands of } \\
\text { the subjects and } \\
\text { sustaining a focus on } \\
\text { academic language }\end{array}$ & $\begin{array}{l}\text { Engaging students in } \\
\text { quality interactions } \\
\text { that promote } \\
\text { productive and } \\
\text { receptive skills }\end{array}$ \\
\hline $\begin{array}{l}\text { Summarising key points from the previous } \\
\text { lesson and checking if students had any } \\
\text { problems doing their individual tests online. }\end{array}$ & & $\sqrt{ }$ & $\checkmark$ & & \\
\hline $\begin{array}{l}\text { Responding to a student's question by } \\
\text { setting up group-work discussion. }\end{array}$ & & $\sqrt{ }$ & $\checkmark$ & & $\checkmark$ \\
\hline $\begin{array}{l}\text { Introducing the lesson and providing } \\
\text { students with some study questions. }\end{array}$ & & $\checkmark$ & $\checkmark$ & & \\
\hline $\begin{array}{l}\text { Using the questions to elicit information } \\
\text { from students and explaining the key } \\
\text { concepts and processes summarised in } \\
\text { slides. }\end{array}$ & & $\checkmark$ & $\checkmark$ & & $\checkmark$ \\
\hline $\begin{array}{l}\text { Using three video clips to facilitate students' } \\
\text { discussion on the business concepts and } \\
\text { procedures. }\end{array}$ & & $\sqrt{ }$ & $\checkmark$ & & $\checkmark$ \\
\hline
\end{tabular}


In summary, Nancy enjoyed participating in the programme and teaching through English as she believed that she could improve her language skills. Despite having some concerns about using English to teach, she gained more confidence through the practice of teaching and learnt some helpful teaching techniques from the EMI course. She tried to use as much English in class as possible while also paraphrasing definitions of business terms in understandable language for students.

\subsubsection{The use of digital technologies}

Nancy said that she needed a number of different technologies to support her teaching (see Table 23). She felt confident in using PowerPoint, the Internet and online search tools as well as the LMS for different teaching purposes.

\section{Table 23}

Nancy's use of digital technologies to support her teaching

\begin{tabular}{|l|c|c|c|c|c|c|}
\hline \multicolumn{1}{|c|}{$\begin{array}{c}\text { Digital } \\
\text { technology }\end{array}$} & \multicolumn{3}{c|}{ The Internet } & LMS & PowerPoint & $\begin{array}{c}\text { Computer } \\
\text { software }\end{array}$ \\
\hline & Websites & Google & YouTube & & & \\
\hline $\begin{array}{l}\text { Accessing } \\
\text { information }\end{array}$ & $\checkmark$ & $\checkmark$ & $\checkmark$ & & & \\
\hline $\begin{array}{l}\text { Presenting } \\
\text { information }\end{array}$ & & & & & $\checkmark$ & \\
\hline $\begin{array}{l}\text { Processing or } \\
\text { creating digital } \\
\text { objects }\end{array}$ & & & & & & $\checkmark$ \\
\hline $\begin{array}{l}\text { Gaming or } \\
\text { interactive } \\
\text { programmes }\end{array}$ & & & & $\checkmark$ & & \\
\hline $\begin{array}{l}\text { Communicating } \\
\text { or collaborating }\end{array}$ & & & & $\checkmark$ & & \\
\hline
\end{tabular}

Nancy used slides to present summarised content every lesson. She included visual information such as graphs, charts and video clips, methods she saw as both convenient for her as a teacher and useful for student learning. She explained that she could "revise the slides easily after each course and reuse them" and that she "provide[d] students with these slides so that they can take notes and use them to review at the end of the course".

Nancy said that she accessed different resources from the Internet. She preferred using Google as a search tool because she could access teaching resources such as multiple-choice questions or quizzes, and even a test bank on the Internet. She complained that her school did not "accommodate the teacher with the test bank provided by the textbook publisher", resulting in much time and effort invested in looking for teaching materials.

Access to YouTube offered Nancy a selection of video clips relevant to her subject that students could use to supplement their business content. She selected clips that were short and 
focused students on the content of the lesson. She felt that students became more engaged and motivated when watching those clips:

I have been looking for clips on YouTube which are in English and subtitled so that students can understand them. I make some comprehension questions and ask students to work in groups. They watch the clips and discuss in their groups to find out the answers.

Nancy said that her subject involved introducing students to technological tools and guiding them on how to use these tools in business. Integrate of such technologies into her teaching activities was therefore important, as the following example illustrates:

To help students understand the benefits of e-commerce, I had the students work in groups. They had to compete to find out information about a trip from Vietnam to Australia. They were asked to search for everything including the best fare price, airlines, accommodation, and appropriate time or activities. After listening to their reports, I gave feedback and recommended to them some necessary tools that could be applied in that case.

Nancy believed that such activities would stimulate students' creativity and encourage them to carry our practical learning activities, as well as cultivating their knowledge and ability to problemsolve. She did not waste time giving students' instructions on using technological tools. Instead, she reported making a visual clip guiding them how to use the tools, explaining that this saved a lot of time in class.

In her opinion, the e-learning system at the university had the potential to do wonders for her course. Not having used any e-learning systems before, she felt satisfied with the current LMS. She uploaded lesson slides, video cases, instructional clips, and all relevant materials for students' reference. She also integrated a range of activities so students could study her subject effectively, as she explains:

After each lesson, I upload a small quiz or assignment to the LMS for students to do as homework. I also designed some assignments requiring students to use the tools they had learnt to create a drawing or solve a problem. Students had to submit their answers on the LMS where their friends could give comments.

Nancy stated that she always encouraged students to use digital technologies in their study. She thought that this would be helpful for students as their major was about using technology in doing business. Specifically, she wanted students "to be familiar with technological tools and gain confidence in the ability of using technologies in an abundance of activities".

In summary, Nancy used a range of digital technologies in her teaching. She accessed the Internet for different resources, and designed slides to present summarised content to students. She integrated digital technologies into her teaching activities to facilitate students' learning and 
encouraged them to use technological tools and devices to solve practical problems or deal with simulated business situations.

\subsubsection{The students}

Seven students participated in a focus group: three males and four females. They expressed different levels of confidence in three areas of content knowledge, English competence and digital technology (see Table 24). Six of them said that they were not good at English and that they did not have any confidence in using English for either communication or study. The other student felt confident in his reading, writing and speaking skills but mentioned that listening was a big challenge for him in studying English. All students reported using digital technologies frequently not only in daily life but in learning as well, believing that these tools played a pivotal role in their learning. They said that they commonly used technologies like laptops, mobile phones, web-based tools, the Internet, social networks or applications for a range of purposes.

\section{Table 24}

A summary of students' profiles regarding their confidence from the most (1) to the least (3)

\begin{tabular}{|l|c|c|c|c|}
\hline \multirow{2}{*}{ Students } & \multirow{2}{*}{ Gender } & \multicolumn{3}{|c|}{ Field of confidence } \\
\cline { 3 - 5 } & & Content knowledge & English competence & Digital technology \\
\hline Student 1 & Male & 2 & 3 & 1 \\
\hline Student 2 & Male & 2 & 3 & 1 \\
\hline Student 3 & Female & 2 & 3 & 1 \\
\hline Student 4 & Female & 1 & 3 & 2 \\
\hline Student 5 & Male & 1 & 2 & 3 \\
\hline Student 6 & Female & 1 & 3 & 2 \\
\hline Student 7 & Female & 2 & 3 & 1 \\
\hline
\end{tabular}

None of these students had learnt any subjects through the medium of English before. However, they had some experience looking for information in English to do assignments for other subjects. Student 2 mentioned "search[ing] for some reference materials written in English on the Internet when writing an essay related to microeconomics", for example.

Five students stated that they decided to study their subject in English as they wanted to improve their English skills for future semesters when they would be required to study many economics subjects in English. The other two students said that initially they had refused to take part in the course taught in English but then were persuaded by teachers and their friends. Another reason these students took the course in English was that they realised that a great deal of information related to computers and technology in the subject is published in English. 
While students thought that they could benefit from studying the subject through English, they found this such a sizable challenge that they "had to stay highly focused and invest much effort". The student whose weakness was in listening said that he gained some confidence in that skill thanks to listening to the teacher in class. They all believed that their English competence would improve through the EMI process, as stated by Student 2:

I can expand the range of vocabulary related to my major. There are many words in the area of information technology I have met before, but I do not understand them. I now realise what they mean when listening to others. Besides, I am exposed more to English in class and through doing tasks or assignments, so I feel more confident with my English.

Nevertheless, these students shared concerns about studying the subject through English. They felt afraid that they did not have enough vocabulary to understand business terms translated from English into Vietnamese and found it hard to ask for help from others, as expressed by student 3: "This is a new programme, so I cannot find any relevant materials, exercises or advice from senior students". Some students also complained that they could not stay focused during a three-hour lecture taught in English, adding that listening to English required so much effort that they felt exhausted by break time.

All students said that they had to work hard to study the content knowledge through English. They reported reading the textbook and reviewing the slides frequently to understand business terms and concepts. Four students said that they tried to memorise English business terms and translate business definitions into Vietnamese. One student stated that he had to translate every single business concept into Vietnamese before he could understand: "With some abstract concepts, I have to review English definitions, focusing on the grammatical points and the way in which terms are explained so that I can memorise definitions in English".

Regarding the teacher's instruction, all students thought that her use of both languages in class was helpful for them:

I always try to focus on the teacher's lecturing in English, but when I feel too tired to stay focused, I only pay attention to her Vietnamese. Sometimes I can improve my English by linking between the teacher's use of English and Vietnamese.

This suggests that bilingual lectures helped Nancy differentiate her instruction to accommodate students with different levels of English.

All students believed that doing practical exercises helped them revise the class theory, adding that the questions or homework assigned by the teacher after each lesson encouraged them to work harder. They also felt motivated by the teacher's use of video clips in the lessons: 
Watching clips and discussing in groups in preparation to answer the teacher's questions were an exciting part of the lectures. Those clips helped change the atmosphere of the class and allowed me to stay alert. I love listening to the voice of native speakers in the clips. Those clips also summarise key points of the lessons in an easy way to understand and memorise.

However, some students complained that they could not keep up with the speed of the speakers in the clips. They said that they understood the content by connecting images and sound or guessing from visual information.

All students agreed that they needed to use digital technologies to assist them in learning the subject through English (see Table 25). They reported that they most commonly used mobile phones and laptops. These devices connected to the Internet so that they could utilise a range of web-based tools and programmes. Student 6 explains:

I used the Internet on my laptop to access Google, a popular searching tool that provides me with links to varying sources related to my subject. I often surf academic journals websites or magazines about information technology.

In addition, the students installed many applications on their mobile phones such as dictionaries, translation apps and e-book readers so that they could look up words or do further readings. They also mentioned YouTube as a useful source of clips related to their subject and they reported watching and downloading many clips from YouTube that provided them with instructions of how to use different technologies in business. Student 5 explained: "I could turn on English or Vietnamese subtitles when watching those clips". All students agreed that the functionality of video clips aided their understanding and ability to memorise new content. 


\section{Table 25}

Students' use of digital technologies in the digital age learning matrix (Starkey, 2011)

\begin{tabular}{|c|c|c|c|c|c|c|}
\hline Aspects of learning & Doing & $\begin{array}{l}\text { Thinking about } \\
\text { connections }\end{array}$ & $\begin{array}{l}\text { Thinking about } \\
\text { concepts }\end{array}$ & $\begin{array}{l}\text { Critiquing and } \\
\text { evaluating }\end{array}$ & $\begin{array}{l}\text { Creating } \\
\text { knowledge }\end{array}$ & Sharing knowledge \\
\hline $\begin{array}{l}\text { Explanation of aspects } \\
\text { of learning: }\end{array}$ & $\begin{array}{l}\text { Isolated } \\
\text { information. } \\
\text { Focus on } \\
\text { completing a } \\
\text { measurable task. }\end{array}$ & $\begin{array}{l}\text { Connecting } \\
\text { thinking. } \\
\text { Simple } \\
\text { connections made } \\
\text { within a context. } \\
\text { Compare and } \\
\text { share. }\end{array}$ & $\begin{array}{l}\text { Develop } \\
\text { conceptual } \\
\text { understanding of } \\
\text { 'big ideas' }\end{array}$ & $\begin{array}{l}\text { Evaluating and } \\
\text { critiquing to } \\
\text { explore the } \\
\text { limitations and } \\
\text { potential of } \\
\text { information, } \\
\text { sources or } \\
\text { process. }\end{array}$ & $\begin{array}{l}\text { Creativity - } \\
\text { applying ideas, } \\
\text { processes and/or } \\
\text { experiences to } \\
\text { develop a new } \\
\text { reality. }\end{array}$ & $\begin{array}{l}\text { Sharing the new } \\
\text { knowledge through } \\
\text { authentic contexts } \\
\text { and gaining } \\
\text { feedback to measure } \\
\text { value. }\end{array}$ \\
\hline Accessing information & \multicolumn{3}{|c|}{$\begin{array}{l}\text { Students used the Internet (Google) to search for information } \\
\text { from magazines, journals or YouTube so that they could } \\
\text { understand the business concepts. }\end{array}$} & & & \\
\hline $\begin{array}{l}\text { Processing or creating } \\
\text { digital objects }\end{array}$ & \multicolumn{6}{|c|}{$\begin{array}{l}\text { Students surfed websites for information they needed to do the exercises, then came up with answers and uploaded these to } \\
\text { the LMS for the teacher's feedback and friends' comments. }\end{array}$} \\
\hline $\begin{array}{l}\text { Gaming or interactive } \\
\text { programmes }\end{array}$ & \multicolumn{3}{|c|}{$\begin{array}{l}\text { Students did the quizzes on the LMS to understand the } \\
\text { business concepts. }\end{array}$} & & & \\
\hline $\begin{array}{l}\text { Communicating or } \\
\text { collaborating }\end{array}$ & \multicolumn{6}{|c|}{ Students joined groups on Facebook to exchange information with others and contacted the teacher through the LMS. } \\
\hline
\end{tabular}


All students reported using the LMS as required by the teacher. Despite not being interested in the system, students felt engaged in the activities that the teacher assigned, as evidenced in this quote:

The teacher uploads questions or assignments onto the LMS after every lecture, so I have to access the page weekly. I can download some guiding clips or case studies to review the lessons. I often log onto the LMS to stay updated on announcements from the teacher, too.

Yet students also had some reservations about using the LMS. For example, Student 7 noted that, "the page is not very user-friendly and lacks many vital functions" As well as wishing that the teacher posted clips of her lectures, the students a would have also liked to receive notifications of any new updates on the system, and have access to the tools to contact the teacher and receive instant responses like on Facebook.

Facebook appeared to be a social network popular among the students. Four students in the focus group said that they were members of groups on Facebook and these did wonders for their subject study. Student 4 explained:

I can exchange information with other members. I will post questions on the group's page so that I can get advice from others including experts in the field of business technology, foreign students or businessmen around the world. That is why I can use English with them sometimes.

In general, all students said that learning in English provided them with higher motivation and inspiration. Three students had grown more confident in their English and had become interested in studying English. One student said that the programme was an opportunity to access a high-quality programme without paying high tuition fees. Some students had suggestions for the teacher. They preferred more explanations added to English slides and learning activities enabling them to "compete or debate with each other or share opinions and solve problems".

In summary, students in the focus group were not confident in their English proficiency but felt motivated to study through English and believed in the programme benefits. They worried that their limited English competence might inhibit their understanding of content and they tried to work hard at home. All students felt they gained more confidence when the teacher used bilingual instructions in class. They perceived the usefulness of digital technologies in providing them with access to information and resources, seeking support from other people in social groups or forums, and facilitating them to improve their English and gain understanding of business concepts. 


\subsubsection{Summary}

Nancy and her students appreciated working in a context where English was used as a medium of instruction. Nancy wanted to build her own brand as a specialist teacher in both English and Vietnamese. She felt pleased with the pay and working conditions offered by the university. Her students believed that the programme would allow them to improve their English and become competitive in international contexts.

Nancy had little experience in teaching economics through English. She felt worried at the beginning but was becoming more confident through practice. In addition to changing her teaching methods to try and make her lessons more effective, Nancy had strong knowledge in her field, which gave her confidence in teaching the subject matter. She used both English and Vietnamese in class and was confident in her use of English. However, to compensate for her students' low English levels, she had to translate when giving lectures. Nancy integrated digital technologies into her teaching with assurance to access or present information and also to facilitate her teaching activities.

The case study students worked hard to understand this new subject. They said that they had to prepare lessons at home and invested time in doing exercises but found it difficult to memorise many business concepts. None of the students felt that their levels of English were high enough. They reported spending more time dealing with the content in English than in Vietnamese. They first thought that English would prevent them from understanding lessons, but then recognised improvement in comprehension and range of business vocabulary. They were fully aware of the benefits of technological devices in their learning and accessed a range of sources of information and reference materials on the Internet as well as joining online groups and forums to seek help from others.

\subsection{Chapter summary}

Each case illustrates different teacher and student EMI experiences. While all teachers possessed strong content knowledge, they expressed different levels of confidence in their ability to teach their subject through English. Each of them used different strategies to teach the subject matter, to use English to give instruction, and to integrate digital technologies in EMI practices. All students participating in the study, albeit with a clear overall preference for the EMI programmes, struggled to understand content knowledge in English and improve their English proficiency. 
However, they possessed high levels of digital competence reflected through a range of digital technologies in their learning activities. 


\section{Chapter 5. Cross Case Analysis}

This chapter addresses four themes identified in the process of analysing data across the four cases. Data were abductively analysed for themes in four phases including: initialisation, construction, rectification, and finalisation (Vaismoradi et al., 2016). In the first phase of initialisation, the codes were identified, labelled, and scrutinised to prepare for further analysis. In the second construction phase, the codes were compared across cases, classified, and grouped as categories for the construction of potential themes. In the rectification phase, potential themes were considered in relation to the literature and research questions. Finally, the themes were developed to gain a full understanding of the participants in the studied context. The four themes identified through this process include: An expectation - reality gap; Pedagogical decisions; Integration of content, English and digital technologies; and Developing autonomous learners. Key findings from the cross-case analysis were then summarised in response to the research question at the end of the chapter.

Each theme reflects insights into teachers and students' practices in English-medium instruction (EMI) courses. The first theme reveals an expectation - reality gap that the teachers and students experienced in the emerging context of EMI courses. The participants held different expectations about joining EMI programmes, but then realised there were mismatches in practice. The second theme focuses on the pedagogical decisions that the teachers made teaching economics subjects through English. It investigates how and why they adjusted their teaching to support students' learning in the new context. The third theme captures how their efforts to integrate English as the medium of instruction and digital technologies into teaching their subject impacted the teachers' pedagogical decisions in EMI courses. The final theme looks at how the students in the study developed into autonomous learners in the new EMI environment. Three aspects of autonomy are explored in detail: student agency, student digital competence, and student personalised learning networks.

\subsection{An expectation - reality gap}

An expectation - reality gap is the difference between what teachers and students expect before participating in academic programmes and what they experience in reality (Winstone \& Bretton, 2013). This theme captures participants' expectations and the challenges they faced during the implementation of the programmes where English was used as a medium of instruction. Identified gaps are addressed from data across four cases not only to show the common experiences 
but also the unique features of each case (Miles et al., 2013). Two sub-themes look at the teachers' and students' experiences.

\subsubsection{Teachers' expectations and reality}

All teacher participants alluded to certain expectations they had brought with them when deciding to teach their course in English. A mismatch occurred between their expectations and reality regarding professional development, the provision of teaching and learning materials, and the students' language proficiency.

\subsubsection{Professional development}

The teachers expected that they would receive comprehensive training in how to use English as a medium of instruction. They anticipated that there would be specific guidance related to the pedagogy of teaching disciplinary subjects through English. Vincent, for example, relays this expectation in his statement: "The university has organised some courses in EMI for teachers who want to teach their subjects through English. I have registered to take the course and hope to learn useful teaching methods".

These EMI training courses, however, did not meet their expectations as the focus was only on providing teachers with language phrases that they could use to give instructions. Nancy did not feel satisfied with the course and thought that other teachers who had studied in English speaking countries would share her feelings:

The course was somewhat useful for teachers who had not had opportunities of studying abroad. I have not used many of the strategies introduced in the course, as they are mechanical and unnatural. I just speak English as naturally as communicating in real life.

Nancy had expected that the course would offer her a range of teaching techniques or strategies but realised that there was not sufficient emphasis on EMI pedagogy. Taylor and Nathan had the same experience in that they had hoped to receive advice on teaching through English rather than learning to use English. While three teachers shared their disappointment, Vincent (who had not attended the training course) believed that this would give him useful tips in using English and teaching methodology. What disappointed him was that the EMI courses were not run frequently enough to meet the demand for EMI teachers. Vincent was not able to gain a place on the course and complained about the length of time spent waiting, saying that "they only run it when there are enough participants". 
In summary, all case study teachers had hoped that their participation in the programmes would be a great opportunity for their professional development in teaching with EMI. However, the lack of focus on EMI pedagogies and the limited number of training courses seemed to cause a mismatch between teachers' expectations and their experiences of the programmes.

\subsubsection{Teaching and learning materials}

The university had selected teaching and learning materials before launching the programmes, deciding to use foreign publications of English course books used at overseas $\mathrm{HE}$ institutions. All the teacher participants were involved in the process of designing curricula for the Advanced and High-Quality programmes. They discussed the content with other colleagues to select appropriate chapters for the courses and translated those chapters into Vietnamese so that the curricula could be assessed and approved. Although they were familiar with the content of those course books in English, they indicated that one source of discrepancy between their expectations and reality related to the availability and accessibility of those materials, as Vincent shared:

I only have a scanned version of the course book. I have provided students with that version. There are only two or three books available in the library for students to make copies, which is somewhat controversial due to the copyright issue.

All the teachers expressed a common concern about the quality of the copied course books and worried that using copies could hinder students' understanding. Vincent specified that "[the] images and figures in tables or graphs are too dark or blurred for students to see, which can decrease their excitement in learning".

The teachers spoke at length about their experiences preparing teaching and learning activities for their lectures, and clearly expressed how the lack of reference resources and materials did not match their expectations, as evidenced by Nancy:

The course book package includes case study videos, teacher instructions and an online test bank. The university only purchased the main course book, so it took me time to go online and look for appropriate videos, write case studies, design activities and compose test items.

Like Nancy, Vincent had to spend time searching for resources, including solutions to case studies suggested in the course book. They both complained about the unavailability of digital resources like online test banks or video cases for their teaching. Nathan and Taylor were lucky as they could access the teacher's books for their subjects. 
In summary, the teacher participants had some concerns about the teaching and learning materials. In this matter, their expectations had been raised by their previous experience of being supplied with materials for teaching and assessments. Therefore, they felt insufficiently supported.

\subsubsection{Students' language proficiency}

The policy makers anticipated that the English proficiency of participating students would be adequate to meet the demands of the courses taught in English. Teachers, too, felt that students' English proficiency was likely to be adequate for the EMI courses, as summarised by Nathan below:

The students in the High-Quality programme were believed to be qualified as they sat a placement test in English when they started their first year. Besides, English modules were considered as a support for students in terms of language.

Even though the teachers had imagined that students' levels might vary, they all spoke of how the students' proficiency in using English was much more limited than they had expected. They viewed this expectation-reality gap as a significant challenge. Nancy stated that most of her students had "limited English skills, especially speaking and listening." She believed that she needed to translate her instruction into Vietnamese to make students understand. Vincent and Taylor found it hard to use English and to design activities to accommodate different English levels in class. Taylor explained:

Students have different levels of English. Many students can barely understand the lectures if I use English all the time. A few students with good English feel bored if I speak too slowly and repeat things several times.

All teachers thought that the university's policy did not provide an adequate evaluation of the students' language level on entry. Two groups of students participated in my study including firstyears in one case and third-years in three other cases. The third-year students had not been asked for any evidence of English proficiency except for taking some compulsory English modules. Meanwhile, first-year students were put into classes according to their English scores in the high school graduation examinations, which did not assess students' preparedness to learn business subjects in English. This created pressure on both the teachers and students participating in the programmes.

The teachers all suggested the policy makers should have "set certain requirements in English for students who want to join in the programme" (Taylor). They believed that "a language support scheme was an essential part in the implementation of these programmes" (Nathan). 
In summary, a gap existed between the teachers' expectation about students' English competence and their actual English proficiency. The teachers had anticipated a high level of student competence in English, so they struggled to scaffold their subject and communicate in English with their students. Not only did they have to deal with students' limited English proficiency but they also had to manage the different levels of English in class. Thus, the teachers suggested that a language entry requirement should be set for students wanting to join the programmes.

\subsubsection{Students' expectations and reality}

All focus group students spoke about their expectations of EMI courses. Some expectation reality gaps were revealed when they discussed their experiences of how they had prepared for the courses and what they were able to achieve.

\subsubsection{Students' preparedness}

Student participants had thought that they were prepared to take the courses in English and expected to receive adequate and ongoing support for their English skills from both lecturers and the university. However, both the first-year and third-year students who joined in focus group discussions shared a growing realisation of their actual preparedness for the course, as stated below:

This is the second subject I have had to learn through English, so I am not used to it. I have realised that I should have started exploring economics content in English earlier. The lecturers only focus on content knowledge while English seems to be a big barrier for us. (Student 1 Focus group 1)

The students who were in their third year were on the one hand expressing a desire for exposure to English in class, but on the other finding it hard to process information in English. They realised that their English proficiency was barely adequate for the new learning context. They also felt disappointed with the English modules that they had been taking alongside their main subjects in economics, as evidenced in this quote:

I have not focused on the studying of English since my first year. I first thought that this course could help me improve my English, but then realised that I have been struggling with both content knowledge and English. I cannot catch up with other friends in my English classes. Thus, I always feel bored and only try to pass those English modules. (Student 2 - Focus group 3)

Many students thought that their English modules were not effective as they noticed that the teachers did not know how to accommodate students of different levels in the same class. This led them to conclude that "the lessons are often boring and passively taught" (Student 3 - Focus group 
1). Students reported having to study the same grammar points again and again. The students in the study had complementary skills and expectations. The first-year students joined the EMI programmes confident in their English proficiency as they had been selected for their high scores in English exams. They shared the same expectations with their senior peers but revealed that business terms and background knowledge in content subjects were a challenge:

Studying in English is exciting, but very hard, particularly understanding economics knowledge. I have never learnt about economics before. Many [English] words have different meanings in economics, so I always feel confused. (Student 1 - Focus group 2)

All the students discussed the expectation-reality discrepancy in terms of the overwhelming content to learn and the concentration this took. Many students had enrolled in multiple different economics subjects at once even though they had not learnt these through English before, as described in the following quote:

Last week, I had to study two chapters in one lecture, which was too much for me. I could not understand the second chapter that the teacher lectured on after break time. Studying three major subjects is overwhelming as the subject issues are hard to understand even in Vietnamese. Now I have to process them in English. (Student 7 - Focus group 1)

Some students complained that the teachers spoke very fast to get through the material and even skipped some parts in the coursebook. They struggled to keep up with the teachers' speed in the lectures. Other students, especially the first-years who were new to the learning at university level, felt exhausted when they had to listen to long lectures delivered in English:

I had read the chapters at home, so I felt excited and understood the lesson very well. Then listening to a lot of English and concentrating for one and a half hours made me feel too tired to follow what the teacher was saying. (Student 2 - Focus group 2)

The students said that intense concentration made them so exhausted and sleepy that they could hardly understand anything even when the teachers used Vietnamese rather than English.

In summary, the students shared their similar experiences between what they had prepared for and what they actually experienced during the courses. They discovered that their preparedness in terms of English, content knowledge and concentration was not adequate to cope with the intensity of learning their subject in English.

\subsubsection{Dual achievements in content knowledge and English competence}

All the students held high expectations that they would improve in both content knowledge and English skills when taking the courses and some students believed that the core objectives of the 
courses were designed to help them in both. They also perceived that they would learn simply through being exposed frequently to English from classes with their lecturers. These expectations contrasted sharply with what they experienced in class, as indicated below:

To understand content knowledge, I have to translate the lesson into Vietnamese and learn it in Vietnamese. Even though I want to improve my English, I cannot understand if the teacher uses English a great deal in class. (Student 4 - Focus group 1)

Some students, especially those with good English, worried about the teachers' inaccurate English pronunciation or intonation. They thought that these might prevent them from fully understanding the lessons. While discussing the challenges presented by learning in English, students spoke of how language affected their content learning:

Some terms are difficult to explain in English, so the teacher only repeats what is written in the book without thorough explanations or clear examples. This restricts our understanding of content knowledge and practical information. (Student 3 - Focus group 3)

A few students reported reading a course book written in Vietnamese so that they could be clear about the business concepts. They believed that their range of business English vocabulary improved largely thanks to their reading at home, rather than learning from the teacher.

In summary, students had expected to improve both in English and content knowledge, but this was not what they experienced. Lack of English objectives in the lectures and the teachers' limited English proficiency presented barriers to their learning both of content and of English.

\subsubsection{Summary}

This theme has explored some EMI course expectation - reality gaps that the teachers and students revealed during the study. The teachers' experiences did not match their expectations of three areas: professional development, the provision of teaching and learning materials, and the students' language proficiency. In particular, the teachers felt insufficiently supported due to little focus on EMI pedagogy training or few training courses, the lack of reference resources or low-quality materials, and students' limited English competence and the varying levels of English among students in their classes. From the students' perspectives, they reported a mismatch between their preparedness for the EMI courses and their content learning and English proficiency. While the subject curriculum was intense, the students realised that they did not have sufficient English to learn the subject matter in EMI courses without a great deal of extra effort. What was worse, the students 
reported that they were unable to improve their English skills and learn economics content at the same time.

\subsection{Pedagogical decisions}

In Shulman's (1987) model of pedagogical reasoning and action, pedagogical decisions are a series of choices and actions that teachers conduct in the teaching process. Based on a digital age perspective, Starkey (2010) modified the model to emphasise teachers' decisions made during the teaching process. These teaching decisions include comprehension of subject knowledge, enabling connections to prepare for teaching, teaching and learning of new content, reflection on teaching decisions, and new comprehension of the subject, students, and teaching (Starkey, 2010). Moreover, in programmes associated with content and language integrated learning (CLIL) or content-based teaching/instruction ( $\mathrm{CBT} / \mathrm{CBI}$ ), teaching and learning through an additional language is itself a specialised process and requires specific research-informed pedagogies (Walkinshaw et al., 2017). Teachers in these contexts (such as those in this study's research context) are expected to adapt their pedagogical decisions to accommodate teaching in English and teaching that integrates digital technologies. This theme addresses what the case study teachers had planned to do in their lectures and how they conducted their planned activities, then highlights the reasons they offered for these pedagogical decisions. Four sub-themes were identified in the data to discuss the teachers' teaching activities.

\subsubsection{Making new content accessible to learners}

In my study, the teachers paid close attention to students' understanding of subject matter. They looked for appropriate strategies supporting students to access new content effectively. In other words, they tried to make subject matter accessible to their students.

All the teachers looked for practical examples to illustrate important points in their lectures. They selected examples that were closely related to Vietnamese contexts or reflected current situations in Vietnam in order to help students understand the lessons. Nathan, for example, decided to replace examples given in the course book with those more likely to be comprehensible to his firstyear students, who did not have a background in economics. He explained that because the course book was not written in Vietnamese, the examples were not related to Vietnamese contexts. He supported students by trying to make connections with students' prior academic learning. Nancy also felt that examples had to reflect students' daily lives so that they could use their classroom knowledge 
to explain contexts familiar to them. This would not only motivate them in learning but also help them apply what they have learned and enhance their retention, as detailed by Nancy below:

To define inflation, I discussed with students about price changes in the market. I asked them about Grab and Uber to illustrate the point of applying technologies in business. These cases are very popular among students, so they can actively engage in the lesson.

Taylor also shared her experience of selecting examples to enhance student engagement. Her examples involved show business celebrities, about whom students are often curious. She believed that motivating examples such as these would help both increase students' participation and enhance their understanding. As such, Taylor continued to stay up to date with content knowledge and the latest trends of interest to students so that she could include these in her lectures.

In summary, all the participating teachers tried to make new content accessible to their students using meaningful and practical examples. They were aware that teaching subject matter and using materials in English were constraints on students' understanding of content knowledge. This prompted the teachers to look for interesting ways of presenting content to their students.

\subsubsection{Differentiating teaching}

In my study, the teachers were aware that their students had different background knowledge, levels of readiness, language proficiency, and learning preferences. This required them to adjust their teaching and find appropriate strategies to engage all students. The sub-theme of differentiating teaching discusses how the four teachers employed differentiated instruction to assist their students' learning.

All the participating teachers reported their students' different background knowledge. While Nathan remarked on his students' background in economics, the three other teachers had concerns about their students' different levels of English. Nathan knew that his first-year students were experiencing a challenging transition from high school classes to the university environment. Nathan believed that this was a significant factor he needed to consider in his teaching. Further, the teachers felt that it was difficult to plan for students with different levels of English, as evidenced in the following quote of Vincent:

Forty percent of students in my class have such a low level of English that they cannot understand the lectures. These students are easily demotivated when learning in English. Those with excellent English account for 20\%, and the rest includes students who can manage to follow the lessons with some difficulty. 
The teachers differentiated the content of their lectures in different ways. All of them planned learning objectives for their students and selected the most appropriate content for students' levels. Vincent, Taylor and Nancy selected foundational knowledge which could prepare students for later subjects. However, Nathan thought that the whole syllabus in his subject was necessary to equip students with basic knowledge, so he tried to cover all content in the syllabus, seeking simple ways to explain the subject matter. Even though all four teachers agreed that the pressure of following the syllabus could be overwhelming, they used their experience to select manageable content, as explained by Taylor:

The content of the course described in the syllabus is normally too much to cover in nine lectures. From my experience, I know which sections are important to focus during my lectures in class. I go through less important content very quickly or assign students to read at home and discuss in the next lessons.

Nathan and Taylor shared their techniques for explaining difficult concepts to students. They said that they did not simplify the content of lectures, but tried to add examples, link the theory to practice in real life, and use simple English. They believed that English was a language barrier that prevented them from expanding the lessons. Instead, they provided students with supplementary readings so that those with good English could use them for self-study without feeling bored or demotivated.

The teachers also used digital technologies to aid differentiation. They reduced the risk of overwhelming students with content during lectures by including links or access to further content through Facebook, the LMS or their own websites. They incorporated visual information such as tables, graphs, charts, and photos into slides to illustrate business terms or concepts. However, the teachers' decisions to use digital technologies were affected by their confidence and skill in using digital technologies. For example, Taylor shared how her lack of confidence meant that she only used "slides for presenting information and showing images or charts". In contrast, Vincent and Nancy were confident about integrating technologies in their teaching.

Vincent and Nancy noticed a range in their students' English proficiency. Vincent identified three different levels of understanding in English in his class and noted that each group consequently responded to his lectures in their own ways. For example, those with poor English tried to sit at the back of the class, avoided engaging in group discussions or seemed distracted in class. Nancy experienced similar behaviour. Both Nancy and Vincent decided to show their students short video 
clips with subtitles about business concepts instead of just explaining concepts themselves. They saw the English subtitles as particularly useful for comprehension and felt that these video clips captured students' attention effectively and connected to their interests. Nancy explained in detail as following:

My students prefer watching clips to reading books or slides. I often select one-minute clips summarising the key points. It's better than asking them to read a long text as they are not confident in their English. I always show clips in English with subtitles, and students can match visual images and English words to understand the content.

Vincent also extended students with high English proficiency who had expressed a need to explore intensive subject content. He said, "One group of students in my class showed their interest in researching in the field, so I have guided them on how to do research and what necessary theories and journal articles there are for reference".

All the teachers described that they differentiated learning in their classes by adjusting their choice of language. Nathan followed the Dean's requirement to teach only in English. To compensate, he tried to speak slowly and clearly, and repeated his explanations frequently. Meanwhile, Vincent and Taylor said that they switched into Vietnamese when they explained abstract or difficult concepts or when they realised that students were not following their lecture. They believed that students often became distracted or bored when they did not understand the lectures and saw code-switching as a useful comprehension device.

Nancy decided to make her lectures bilingual after discussing language preferences with her students. This meant that she used both Vietnamese and English to make sure that all students could understand the lectures. She reported using Vietnamese deliberately in her lectures and allowing students to use their preferred language. This shows the presence of translation strategy and translanguaging approach in Nancy's practice despite her not being aware of those strategies.

All the teachers used different strategies to teach their students content knowledge. They considered the specific characteristics of their subjects and their class to decide how the content would be presented. Vincent and Taylor preferred using black boards to show slides to students and only used slides for summarising key content of the lectures. Taylor explained that students needed to follow how she did practical calculation exercises step by step on the board. Meanwhile, Vincent described an activity he used to engage students more effectively:

I asked my students to work in groups. Each group had to note down words from the reading text that they thought important, then they shared the words and their meanings to the 
whole class. I combined students' words and explained the relevant business concepts. I finally showed slides summarising key concepts and required content in the lectures.

Nathan chose group activities, as he believed that they would help students exchange ideas and become more confident in contributing to the lectures. Similarly, Nancy designed group discussions for students in her class when she saw that they were energetic and engaged well in the lectures. She also reported that there were students who could not keep up with their classmates, so she had to use different kinds of instruction:

When students are interested in a topic, I often use open questions and get them to discuss in groups. I sometimes get them involved in a debate about a specific point. I make sure to upload a consolidation slide or clip to the LMS so that low students can read or view again.

In terms of formative assessment, Nathan used computer-based assessment to provide students with instant feedback and save time. Meanwhile, Taylor used a kind of instant assessment to push students in their study:

There were some students who were lazy in doing exercises, so they only copied their friends. I had to give the whole class quick paper tests or online quizzes after important sections. I marked some of them or just looked through to find out what students did not understand and clarified these points in the next lectures.

In summary, the four teachers in the study reported using different strategies to differentiate their teaching and respond to students' differences. They amplified the content, adjusted the language use, and looked for appropriate strategies to present content knowledge to learners. Digital technologies became supplementary tools assisting them in the differentiation of their teaching.

\subsubsection{Facilitating students' analytical thinking and problem-based learning}

All participating teachers stated that enhancing students' analytical thinking and problemsolving skills was a priority when setting objectives and planning their lectures. Nathan explained:

All lectures target the first aim of making students understand clearly definitions of business concepts. The second aim is to help students to apply their understanding in answering questions or solving problems in their daily life. They should be able to employ economic principles in their real life.

Students were set activities where they needed to use high level thinking to apply business concepts. The lecturers posed a series of questions which required them to read information, analyse data, and connect knowledge to come to a thorough comprehension of subject matter.

Nathan and Taylor used questions during their lectures to encourage students to think logically and analytically about the content. They tended to start lectures with yes or no questions, 
then asked open-ended questions to engage students in discussions as well as build depth. Vincent and Nancy reported that they converted lecture objectives into questions so that students began to think about what they were going to study. They believed that this would trigger students' curiosity and develop their ability to think and analyse, as described by Nancy below:

I start a lecture with questions that focus students on the topic they are going to learn. These questions convey knowledge objectives that students need to achieve at the end of each lecture, so they must think about them during the lecture. I also check if students can answer these questions to make sure that lecture objectives have been accomplished.

All the teachers shared how they developed students' analytical thinking skills in their lectures. Nathan chose visual aids such as charts and graphs to get students to explore key concepts. He accessed graphs and charts online or drew them himself using Excel or Word. He used figures and statistics to prompt students' analytical thinking. Meanwhile, Taylor said that she summarised content in a table and asked comprehension questions. Students then tried to review what they had studied in the lectures and expressed their understanding by answering the teacher's questions. Taylor felt that this strategy reinforced students' learning. In the meantime, Vincent and Nancy reported selecting case study videos to facilitate students' analytical thinking. Nancy described her use as following:

I showed students video clips and got them to answer certain questions. Each clip was actually a case study that required students to think carefully and find out solutions to a specific problem. They discussed in groups and used their developing knowledge to justify their answers.

They both believed that case studies engaged students in problem solving as students had to work on the concepts they had studied and apply them in solving specific problems. Nancy and Taylor said that they encouraged students to explore the problems in their own way as long as they could propose appropriate solutions.

In summary, the teachers engaged students in activities where they could develop their analytical thinking skills through problem-based learning. Questions and visual aids like charts or graphs were used to stimulate students' thinking about new concepts. The teachers also encouraged students to consolidate and review the lessons by connecting key points and summarising them in tables. Case study was reported to be an effective problem-based learning activity especially when students engaged in problem-solving process and found solutions by themselves. The teachers used 
digital technologies such as search tools to give students access to a wide range of resources including graphs, charts, tables, and video clips.

\subsubsection{Engaging students to collaborative and interactive learning}

This sub-theme discusses how the teachers in the study tried to create a collaborative and interactive learning environment for their students. All of them thought that engaging students in activities where they could collaborate and interact with each other frequently would not only enhance students' communication skills but stimulate their subject learning as well.

All the teachers planned group work to ensure student collaboration in their learning. The activities involved group discussions, case study presentations, problem-solving tasks or quiz-show competitions. The teachers identified several key purposes of these group performances including engaging students in learning, enhancing students' interactions, and collaboration.

Nancy believed that students liked working together as they seemed confident talking to their group members before presenting to the whole class. Thus, Nancy frequently designed learning tasks in which students discussed issues in their groups and exchanged their ideas with other groups in class. She believed that this helped to facilitate students' engagement in the learning process:

I got students to form groups at the beginning of the course. They enjoy working in their groups as it is easy for them to exchange ideas and find correct answers. Presenting their group's answers is easier than giving their own answer individually.

Nancy used the computer system in the computing lab to interact with students in some of her lectures. She monitored students' learning activities through the head computer and sent students materials, assigned tasks, gave instructions, and responded to their individual questions.

Taylor and Vincent felt that students' interaction in their classes was still limited. They wanted to involve students in group work so that students could improve their English and develop communication skills. While Taylor struggled to have students interact in groups, Vincent experienced some success in enhancing students' interactions. He involved students in giving feedback to their peers during group work presentations so that "students felt motivated to engage in group and whole-class discussions".

Nathan reported encouraging students to work in groups so that they could help each other. He thought that his first-year students needed to collaborate actively with others both inside and outside class to help them adapt to the new learning environment. Nathan tried to design group activities in class but admitted that they were not very successful: 
The questions I raised for group discussions were too short and simple to stimulate discussion. Another issue is that students coming late grouped together, so all group members could not catch up with the whole class. This has made group discussions ineffective.

All the teachers reported trying to encourage students to interact more with each other and with the teachers. They used different strategies to make learning activities more interactive. Vincent asked students to identify the key content of the lessons and then discuss it with others in class. Vincent confirmed that he "would wrap their discussions up with a recap and some follow-up questions or exercises at the end".

Taylor experienced some difficulties in trying to create interactions, noting that "students often keep silent when I ask them questions", believing this to be because of their limited English proficiency. Therefore, she added an element of pressure by calling on students randomly to answer her questions. However, she felt that this strategy was not ultimately effective due to the potential for student sensitivity to answering questions they were not prepared for, leading to possible demotivation. As an alternative, Taylor tried to create opportunities for students to ask questions. Nancy and Nathan shared the same struggle regarding their desire for students to speak more in class.

All the teachers thought it was important to interact with students outside class. For example, Nathan set aside time every Tuesday and Thursday to meet with students in his office if they needed help. The teachers also used digital technologies to contact students and create a channel for them to communicate with teachers, mainly through email. Nathan mentioned that students were afraid of raising questions in class" and saw email as a useful way to avoid/mitigate this fear. Nancy and Taylor, for their part, were familiar with using the LMS to interact with students after class. Nathan said that he had his own website where students could access reference materials as well as ask him questions. Vincent did the same with his own website and also used Facebook:

Students in my class created a private group on Facebook and added me as a member, so they often raise their questions there or ask for my opinions online. I also give them the answers or upload some further readings and case studies for them to discuss and practise.

In summary, the teachers reported using group activities to try and develop student collaboration and interaction. They thought that this would help students to improve their communicative skills and engage in the learning process. Despite their belief that students' English proficiency inhibited them from interacting much in class, the teachers tried to create opportunities 
and even a level of pressure for students to speak by asking questions and eliciting students' answers. All the teachers also tried to interact with students outside class by using technologies like email, the LMS, websites or social networks.

\subsubsection{Summary}

This theme has discussed the teachers' pedagogical decisions around their teaching of EMI economics courses. Four significant sub-themes were developed from the data: how the teachers made new content accessible to students, how the teachers differentiated their teaching, how the teachers facilitated students' analytical thinking and problem-based learning, and how the teachers engaged their students in collaborative and interactive learning. The different strategies used by the teachers to illustrate their pedagogical reasoning can be seen below (see Table 26). 


\section{Table 26}

The summary of the teachers' pedagogical decisions in an EMI economics course

\begin{tabular}{|c|c|c|c|c|c|c|}
\hline & Sub-themes & Teaching strategies & Vincent & Nathan & Taylor & Nancy \\
\hline & \multirow{4}{*}{$\begin{array}{l}\text { Making new content accessible to } \\
\text { learners }\end{array}$} & $\begin{array}{l}\text { Giving examples from the Vietnamese context or } \\
\text { reflecting current situations in Vietnam }\end{array}$ & $\mathrm{x}$ & $\mathrm{X}$ & $\mathrm{x}$ & $x$ \\
\hline \multirow[t]{2}{*}{1} & & $\begin{array}{l}\text { Giving examples connected to students' background } \\
\text { knowledge }\end{array}$ & & $\mathrm{x}$ & & \\
\hline & & Giving examples related to students' daily life & $\mathrm{x}$ & $\mathrm{x}$ & & $\mathrm{X}$ \\
\hline & & Giving examples related to students' interests & & & $\mathrm{x}$ & \\
\hline \multirow{10}{*}{2} & \multirow{10}{*}{ Differentiating teaching } & $\begin{array}{l}\text { Adjusting the syllabus by selecting appropriate } \\
\text { content }\end{array}$ & $\mathrm{x}$ & $\mathrm{x}$ & $\mathrm{x}$ & $\mathrm{x}$ \\
\hline & & Linking theories to real life practice & & $\mathrm{X}$ & $\mathrm{x}$ & \\
\hline & & Simplifying the language used to give instructions & $\mathrm{x}$ & $\mathrm{X}$ & $\mathrm{X}$ & $\mathrm{X}$ \\
\hline & & Showing video clips with subtitles & $\mathrm{x}$ & & & $\mathrm{x}$ \\
\hline & & $\begin{array}{l}\text { Providing students with extension reference } \\
\text { materials }\end{array}$ & $\mathrm{x}$ & & & \\
\hline & & Speaking English slowly and clearly & & $\mathrm{X}$ & $\mathrm{x}$ & \\
\hline & & $\begin{array}{l}\text { Using code-switching between English and } \\
\text { Vietnamese when giving instructions }\end{array}$ & $\mathrm{X}$ & & $\mathrm{X}$ & $\mathrm{X}$ \\
\hline & & Integrating a translanguaging strategy into lectures & & & & $\mathrm{X}$ \\
\hline & & $\begin{array}{l}\text { Using the blackboard to give instructions and using } \\
\text { slides to summarise lessons }\end{array}$ & $\mathrm{x}$ & & $\mathrm{x}$ & \\
\hline & & $\begin{array}{l}\text { Designing group work to ensure students help each } \\
\text { other }\end{array}$ & $\mathrm{x}$ & $\mathrm{X}$ & & $\mathrm{x}$ \\
\hline
\end{tabular}




\begin{tabular}{|c|c|c|c|c|c|c|}
\hline & & $\begin{array}{l}\text { Using instant assessment to push students in } \\
\text { learning }\end{array}$ & & & $x$ & \\
\hline & & $\begin{array}{l}\text { Using computer-based assessment to provide } \\
\text { students with instant feedback }\end{array}$ & & $\mathrm{X}$ & & \\
\hline & \multirow{5}{*}{$\begin{array}{l}\text { Facilitating students' analytical } \\
\text { thinking and problem-based } \\
\text { learning }\end{array}$} & $\begin{array}{l}\text { Informing students of lesson objectives in the form } \\
\text { of questions }\end{array}$ & & $\mathrm{X}$ & & $\mathrm{x}$ \\
\hline & & Using questioning to elicit students' thinking & & $\mathrm{X}$ & $\mathrm{x}$ & \\
\hline & & Using visual aids (graphs or charts) & $\mathrm{x}$ & $\mathrm{X}$ & & $\mathrm{x}$ \\
\hline & & Consolidating knowledge through tables or mapping & & & $x$ & \\
\hline & & Using case studies & $\mathrm{x}$ & & & $x$ \\
\hline \multirow{5}{*}{4} & \multirow{5}{*}{$\begin{array}{l}\text { Engaging students to collaborative } \\
\text { and interactive learning }\end{array}$} & Using group discussions & $\mathrm{x}$ & $\mathrm{X}$ & $\mathrm{x}$ & $\mathrm{x}$ \\
\hline & & Using group presentation & $\mathrm{x}$ & & & \\
\hline & & $\begin{array}{l}\text { Designing quizzes or problem-solving tasks for group } \\
\text { work }\end{array}$ & & $\mathrm{X}$ & $\mathrm{x}$ & $x$ \\
\hline & & Questioning students randomly & & & $x$ & \\
\hline & & $\begin{array}{l}\text { Using digital technologies (websites, email, the LMS, } \\
\text { Facebook) to interact with students outside of class }\end{array}$ & $\mathrm{x}$ & $x$ & $x$ & $x$ \\
\hline
\end{tabular}




\subsection{Integration of content, language and digital technologies influencing pedagogy}

This theme explores the teachers' pedagogies that developed as they attempted to integrate English as a medium of instruction and digital technologies into teaching subject matter.

\subsubsection{Integrating English as a language of instruction into teaching subject matter}

The subject teachers in the study adjusted their pedagogical strategies in order to teach in English. All the participating teachers supported the rationale behind teaching programmes through English. Taylor and Nancy thought that EMI courses would help students improve both content knowledge and English proficiency so that they could compete in the global job market. Nathan and Vincent mentioned internationalisation in education as a worldwide trend that they needed to follow. Vincent explained:

Teaching through English is basically to internationalise our academic programmes. This has been a growing trend in education in the last decade. The trend will open a new horizon for both students and teachers. That is why I made the decision to be involved in the EMI programmes.

In explaining why she decided to teach her course in English, Nancy expressed her belief that students would understand content knowledge, especially business terms, if her subject was taught in the most dominant language used in business:

Materials used in my course were originally written in English. Vietnamese versions might cause certain problems in students' comprehension. The language used in this area is mainly English, so students can avoid being confused about the terms used in their major when studying them in English.

All the teachers had some difficulties in using English to teach their subjects. While Vincent expressed concerns about making grammatical mistakes, pronouncing words inaccurately or having limited vocabulary, three other teachers felt more confident in their English competence, but admitted teaching through English restricted their fluency and articulateness. Nathan and Taylor said that they could not teach in the same way as they normally did in Vietnamese. Taylor chose to focus on key points and make her explanations as simple as possible. She felt bad when she could not expand the lectures to include more intensive knowledge and information. Likewise, Nathan tried to use the simplest language in his lectures and reported that he was unable to create a comfortable learning atmosphere through humour in English:

When I teach my subject in Vietnamese, I sometimes tell students funny stories or jokes to make them relaxed. However, it is very tricky to do the same in English as my English is not good enough and students cannot understand the stories. 
Nancy tried to maintain a relaxing atmosphere in class by allowing students to use either Vietnamese or English. Although she tried to increase the use of English in class, she gave lectures in both languages. She reported switching between languages to make it easier for students to follow the lectures:

I used to design groupwork activities in which students presented different topics in English. However, it was not effective as students' content comprehension and use of English were still limited, so I decided to lecture in both languages and designed more interactive activities instead.

Vincent shared the same experience as he had to change his teaching activities to teach in English. He realised that students found the lectures in English hard to understand, so he decided to switch into Vietnamese after each slide or each section. He said that the use of English limited the interactions between the teacher and students and among students themselves. He designed different activities to enhance classroom interactions, as summarised in the following quote:

In the first lectures, I used a lot of English and encouraged students to interact in English as well. Nevertheless, they kept silent and responded passively to my activities. I had to give them explanations in Vietnamese, get them to discuss in groups and allow them to use Vietnamese too.

Vincent and Taylor shared the strategy of code-switching in their lectures. They said that the teachers needed to observe the whole class during the lectures so that they could know how students responded to the lectures. These observations helped them choose when to use Vietnamese instead of English, as evidenced by Taylor:

I often use Vietnamese to explain abstract terms or difficult business concepts. I always pay attention to students' facial expressions or behaviours to guess how they feel. When students get confused or bored, I will switch into Vietnamese.

Nathan said that he made a few changes in the EMI lectures compared to those taught in Vietnamese, but he reported slowing down and using more questions and examples in class:

My lectures in English are not much different from the Vietnamese ones, but I try to speak slowly enough for students to follow. I have to t explain the concepts step-by-step and check students' understanding frequently. The issue is that there is too much content to cover.

Like Nathan, the other teachers said that they had to check students' comprehension frequently during the lectures in English.

None of the teachers set objectives for English learning in their lectures. They focused on teaching content knowledge and thought that students would pick up English through class activities. Vincent was the only teacher with some content knowledge about the English language 
as he had a degree in English applied linguistics. He designed activities from which students could learn reading skills:

When assigning students to do a case study, I always get them to practise reading skills by identifying key ideas and summarising the texts. In the opening case, students often deal with questions that require them to skim and scan the text to find out the answers.

The teachers may not have included language learning objectives because their EMI preparation courses had not prepared them to understand the language demands of their subject, or how to teach using the medium of English. They seemed to equate English proficiency with learning vocabulary or business terms rather than any other aspect of language learning. Despite not including objectives for language learning, the teachers designed different activities that had the potential to develop students' English skills. They hoped students would improve their English in the process of participating in these activities. Vincent and Nathan often used question-answer activities at the beginning of each lecture which they believed would help students recall business concepts and terms in previous lessons. Nancy also reported using questions to elicit the meaning of terms:

During a lecture, I focus students on important terms that they need to understand. I make questions related to those terms so that students can understand their meanings and memorise them as well. Some terms that do not have exact Vietnamese equivalents need explaining in detail and supporting with specific examples.

All the teachers said that they made an effort to engage students in quality interactions in class. They thought that interactions would help students not only feel engaged and motivated in the lectures but also improve their English communication skills. However, their conceptions of interactions were more general than those promoted in CLIL/EMI literature. In practical terms, Vincent and Nancy conducted group discussions using case study video clips and guiding questions while Taylor and Nathan had students solve practical exercises in groups. Taylor explained:

Students are not confident in their English to speak in class. That is why I have to make a lot of simple questions and call on students to answer. For quizzes or calculation exercises, students can discuss in groups before reporting their answers.

However, none of the teachers made it compulsory to use English in group discussions. Nor did they construct groups in a way that would require students to participate. Instead, they concentrated on promoting interactions between teachers and students, as summarised by Nancy below:

I encourage students to use English as much as possible in class. However, they can use either English or Vietnamese to answer my questions or discuss in groups. For 
assignments, students are going to get bonus scores if they use English to write their paper.

The teachers prioritised students' comprehension of content knowledge and were satisfied if students could manage general communication in English. Nancy added:

Students' levels of English are not the same, so it is hard to make students demonstrate good English use. I only motivate them to communicate in English and engage them in interactions in which they only need to make themselves understood.

In summary, the teachers entered into the EMI programmes feeling confident that they could support students in learning content knowledge and improving their English proficiency. The lecturers believed that EMI would allow them to develop professionally and follow/be part of the trend of educational internationalisation. However, they lacked any formal preparation for how to teach through the medium of English. Using English to teach content impacted their pedagogical approaches but not necessarily in ways that enhanced their students' learning of the language. Although they did not set any objectives for students' English learning, they designed meaningful activities which they believed would contribute to students' English development. Apart from focussing students on vocabulary learning, they also tried to engage students in interactions to increase motivation and improve their English communication skills. The teachers used the strategy of switching between English and Vietnamese in to support their learners (see Table 27).

\section{Table 27}

The teachers' use of English and Vietnamese in class

\begin{tabular}{|l|l|l|l|l|l|l|l|}
\hline & \multicolumn{2}{l|}{ Vincent } & Nathan & \multicolumn{2}{l|}{ Taylor } & \multicolumn{2}{l|}{ Nancy } \\
\hline Presenting the content learning objectives & & & & & & \\
\hline Guiding students to do tasks & & & & & & & \\
\hline Defining terms & & & & & & & \\
\hline Explaining concepts & & & & & & & \\
\hline Giving examples & & & & & & & \\
\hline Making questions & & & & & & & \\
\hline Consolidating lessons & & & & & & & \\
\hline Answering students' questions & & & & & & \\
\hline
\end{tabular}

English

Vietnamese

\subsubsection{Integrating digital technologies into teaching subject matter}

This sub-theme examines the teachers' pedagogical decisions in response to the integration of digital technologies. More specifically, it discusses how the teachers used and integrated technological equipment into their teaching of content knowledge. All the teachers 
valued the benefits of digital technologies in their teaching, employing them for different purposes in preparing and giving lectures as well as interacting with students (see Figure 7).

\section{Figure 7}

Teachers' use of digital technologies

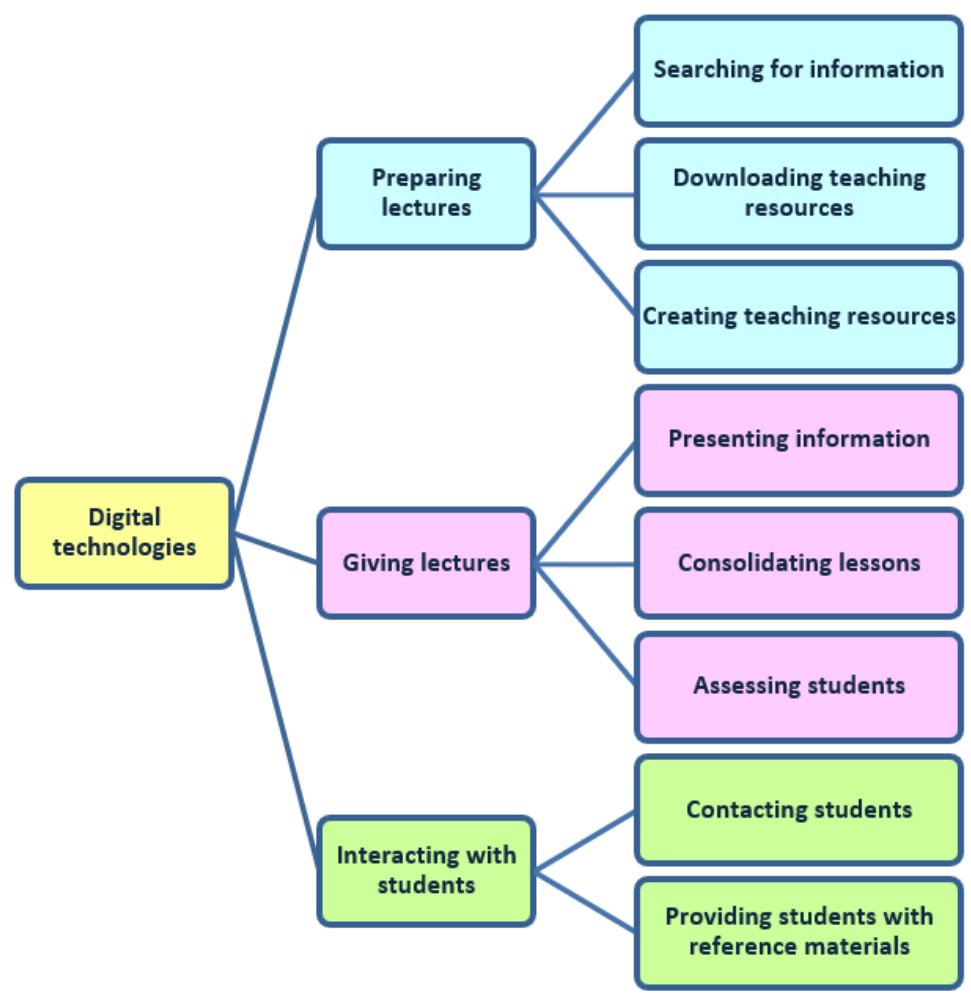

The teachers accessed different sources of information on the Internet to prepare for their lectures. They commonly used Google as a search tool to look for practical examples and update their content knowledge. Vincent and Nancy downloaded YouTube video clips for case study discussions. Vincent and Nathan not only found various visual aids like charts or graphs to include in their lectures, but also drew them using MS Word or Excel on their laptops. Nathan and Taylor designed computer quizzes using i-Spring on the LMS for students to do in class and after class.

In their lectures, the teachers mainly used digital technologies for presenting information. PowerPoint was commonly used for showing definitions of key concepts, displaying visual aids like charts, graphs or tables and summarising major points in the lectures. Nathan and Vincent included many graphs and charts in their slides, and Taylor frequently showed her students a summary table of the whole lesson:

I revise the lecture slides frequently to update the content or examples. My subject involves lots of formulae and related concepts, so I summarise them in a table and show students on slides to help students review the lessons easily. 
Vincent and Nancy presented case studies to students through YouTube clips. They both felt that the clips not only provided students with easy-to-understand information but also helped students improve their English skills with the support of English subtitles, as described by Nancy below:

I start a video case task with some guiding questions. Students then watch the clip twice without subtitles. After watching the clip again with subtitles, students discuss in groups and report the answers. Finally, I show the clip again to explain and assess students' answers.

Nathan and Taylor shared their practice of using online quizzes to engage students in the lectures and to help them consolidate their learning. Nathan also believed that computer-based assessment would bring several benefits to both the teachers and students. He required his students to do mid-term papers on computers so that they were able to "get immediate results" and he could save time from marking their papers. Nancy and Taylor sometimes integrated search tools into students' learning activities, as detailed by Nancy in the following quote:

To teach students about business technology, I had them find the cheapest holiday package in the shortest time. Students worked in groups to present the procedure of looking for information to the whole class. This activity was followed up with a discussion on how to apply digital technologies effectively.

Both teachers thought that getting students to use digital technologies to complete a task or assignment would offer practical experience as well as motivation for students in their learning.

All the teachers reported using different methods to contact and interact with students outside class. Vincent and Nathan frequently used email to respond to students while Taylor and Nancy preferred using the LMS. Vincent was the only one who used a social network for teaching. He valued Facebook as an effective channel to interact with students and for students to communicate with each other:

I got the class' monitor to create a group on Facebook and add everyone in class to the group. I interact with students and attach relevant information or reference materials. Students can post questions to get help from me or their classmates.

The teachers thought that integrating digital technologies helped to manage the overwhelming content of the courses. Vincent and Nathan used their own blog and website to provide students with additional reading texts so that they could save time in class. Similarly, Taylor supported students with links to different online materials that could help students access extra practical exercises, information and relevant forums in their field. Meanwhile, Nancy fully exploited digital resources: 
To guide students how to use a digital tool, I created a manual clip with step-by-step instruction. The clip was posted on the LMS for students to watch. I can reuse the clip in different courses, and students who are not clear about the instructions or are slower than their friends can watch it again and again.

The integration of the LMS into teaching syllabi had an impact on the four teachers' pedagogical approaches. They all included the LMS in their syllabi as required by the university policy. This policy encouraged teachers to offer at least two lectures online via the LMS during their course. While each of the teachers adopted this policy, they did so differently. Whereas Vincent included the LMS in all of his lectures, Taylor and Nancy saved one lecture for online activities. Meanwhile, Nathan expressed little interest in the use of the LMS. However, they all used the LMS to contact students and upload course syllabi, lesson slides, eBooks or reference materials and assignments.

Vincent designed extra activities for students to study on the LMS, attaching these activities to each lecture for students to complete after class. He did not conduct online lectures as he thought that technical issues could disrupt the teaching and learning:

I posted eight different tasks for eight lectures that students had to do on the LMS. Typical tasks include multiple-choice questions, essay writing, case analysis, questions and answers and so on. Students can review the lessons through these tasks.

Taylor and Nancy reported using the LMS for the final assessment. They used the last lecture of the course to conduct an online assessment which involved different activities set at specific times when students could interact with the teachers and complete the requirements. Nancy explained:

In the last lecture, students do not need to come to class. I have set a time and uploaded requirements on the LMS, and students can go online to complete the final assessment. I will be online at a specific time to answer any questions related to the exam as well.

In contrast, Nathan used the LMS for contacting students and providing them with extra materials. He thought teaching online with the LMS would add more jobs for him to do, explaining that teachers' workload was overwhelming and that the LMS was not used consistently across the university. Sharing this opinion, Nancy raised some concerns about the role of the LMS that the policy makers had intended to be a compulsory component of all courses:

Some teachers are used to the Blackboard system, so they find it hard to use the LMS. As it is newly launched, the LMS lacks a lot of functions and is not user-friendly. Some teachers decided to include the LMS to meet the demand of the policy, yet not to integrate it into their teaching properly. 
All teachers reported that they felt hesitant about integrating digital technologies for a number of reasons. Vincent and Nancy had concerns about the availability and accessibility of digital tools although they felt confident in their digital skills. Taylor and Nathan thought that it was time-consuming to integrate digital technologies into their teaching and Taylor explained that she lacked confidence with technology in teaching and designing teaching activities.

In summary, the teachers used a range of digital technologies in different ways in their teaching (see Table 28). Access to various sources of information and materials on the Internet helped them in preparing for the lectures. They were familiar with search tools (Google), social channels (YouTube) and applications on their laptops (MS Word, Excel) which they could use to download or design teaching resources such as charts, graphs, tables, or video clips. The teachers also integrated these digital technologies into their teaching activities in different ways such as presenting information with PowerPoint slides, illustrating the lectures with visual aids, and contacting students via email, the LMS and even social networks like Facebook. These teachers agreed that their integration of digital technologies into teaching was still limited due to the inadequate availability and accessibility of appropriate technology, their overwhelming teaching schedule, or their lack of confidence or digital competence.

\section{Table 28}

The teachers' use of digital technologies in their teaching

\begin{tabular}{|l|l|l|l|l|}
\hline & Vincent & Nathan & Taylor & Nancy \\
\hline Searching for information & $\begin{array}{l}\text { Google, } \\
\text { websites, } \\
\text { online } \\
\text { database or } \\
\text { library }\end{array}$ & $\begin{array}{l}\text { Google, } \\
\text { websites, } \\
\text { online } \\
\text { database or } \\
\text { library }\end{array}$ & $\begin{array}{l}\text { Google, } \\
\text { websites, } \\
\text { online } \\
\text { database or } \\
\text { library }\end{array}$ & $\begin{array}{l}\text { Google, } \\
\text { websites, } \\
\text { online } \\
\text { database or } \\
\text { library }\end{array}$ \\
\hline Presenting information & PowerPoint & PowerPoint & PowerPoint & PowerPoint \\
\hline Downloading video clips & Youtube & & & Youtube \\
\hline Creating video clips & Word, Excel & Word, Excel & & Web-tools \\
\hline Drawing charts, graphs & & & Word, Excel & \\
\hline Designing tables & & i-Spring, LMS & i-Spring, LMS & \\
\hline Creating quizzes & $\begin{array}{l}\text { LMS, } \\
\text { computer lab }\end{array}$ & LMS & LMS \\
\hline Assessing students & $\begin{array}{l}\text { Email, } \\
\text { Facebook, } \\
\text { LMS }\end{array}$ & Email, LMS & Email, LMS & Email, LMS \\
\hline Contacting students & $\begin{array}{l}\text { Facebook, } \\
\text { personal } \\
\text { website, LMS }\end{array}$ & $\begin{array}{l}\text { Personal } \\
\text { website, LMS }\end{array}$ & LMS & LMS \\
\hline $\begin{array}{l}\text { Providing students with } \\
\text { reference materials }\end{array}$ & \multicolumn{2}{|l|}{} & & \\
\hline
\end{tabular}




\subsubsection{Summary}

This theme shows how the integration of English and digital technologies influenced the teachers' pedagogical decisions. In response to using English as a medium of instruction, the teachers reacted by adapting their teaching methods to support students' comprehension. While their EMI professional development had not covered how to teach English or teach through English, teachers used common sense to support their students' learning. They adjusted their ways of lecturing by slowing down their speech, limiting the content and simplifying their language. Further, they designed activities that they believed would engage students in communication and interactions. The integration of digital technologies into teaching activities was also limited but seemed to have an impact on the teaching process.

\subsection{Autonomous learners}

This theme explores the levels of learner autonomy evident in the four case studies. Aspects of autonomy are discussed in detail below as sub-themes, namely student agency, student digital competence, and student personalised learning network. Each of these subthemes in turn is broken down further into categories to highlight how students adapt to the new context of EMI.

\subsubsection{Student agency}

Student agency is generally defined as students' capability to influence their learning environments (Klemenčič, 2017). The agency of students is reflected through a range of possible practices that they conduct to respond to a change in their learning contexts (King, 2000).

Students develop their agency based on their own experience and knowledge of the context to take different learning strategies and approaches that would be more effective to their learning (Thorpe, 2002). This sub-theme discusses how students personalised their learning in the new learning context where English was used as a medium of instruction.

\subsubsection{Independent learning}

Most students in the focus groups shared the common challenges in learning economics subjects through English. Many of them possessed such limited English that they could not fully understand their lectures. Others with high levels of English proficiency had concerns about their teachers' English proficiency and noticed their grammatical mistakes, inaccurate pronunciation or intonation, and Vietnamese accents. They worried that these weaknesses might hinder their comprehension. Some complained about the amount of content they were expected to cover in 
a lecture. All the students said these issues prompted them to engage in self-study at home, as exemplified in the following representative quote:

My English is not good enough, and there is too much content knowledge to learn. I have to invest more time to study at home. I have to prepare the lessons and find ways to comprehend the content by myself because I cannot follow the lectures in class. (Student 4 - Focus group 1)

The students therefore prepared carefully at home before each lecture so that they would be able to understand what the teachers taught in class. Part of this preparation involved regularly reading textbooks in advance using two distinct strategies which neatly divided the participating students. The first group tried to translate reading texts into Vietnamese, with some participants using both Vietnamese and English textbooks at the same. After comprehending the main ideas in Vietnamese, these students read the English texts to compare and understand meanings in English. Meanwhile, the other group of students read the texts exclusively in English, as given in this quote:

I am using an English course book. The teacher has provided us with English slides and lectured in English, too, so I read the texts, take notes and understand business concepts in English. Only when I encounter difficult words do I have to look up their meaning in Vietnamese. (Student 6 - Focus group 2)

Despite using different strategies, all students searched for additional sources of reference to understand significant content. They searched on the Internet for relevant articles, eBooks or video clips on YouTube that could provide them with detailed explanations or practical examples. Some joined Facebook groups and received help from senior students. They believed that preparation at home was essential if they were to follow lectures easily in class, as evidenced in the quote below:

I often have difficulties understanding subject matter in the reading texts. I have to search on Google for relevant information, especially articles, online lectures or video clips. I can find useful advice or thorough explanations in some forums or Facebook groups. (Student 6 - Focus group 4)

Some students complained that there was too much subject content for them to prepare at home. This meant that they were unable to follow parts of the lectures. Another problem occurred when the teachers skipped some sections in the textbooks, which meant that students had difficulty in keeping up with the lectures. Students used particular strategies for learning business concepts in English. The most common was to follow the process of "definition explanation - example". Many students explained how they read the definitions of business concepts, identified key words and checked their meanings to gain a general understanding. Next, 
they would go over the explanations and relevant examples to fully comprehend the concepts. These students thought that identifying key words helped them recall the concepts effectively. In other words, they concentrated on learning key vocabulary, as described in this following quote:

When learning a concept, I always focus on identifying significant words that can help me understand main ideas. I write those words down in my notes and try to use them as prompts to restate the definitions. I only need to memorise key words when preparing for tests or exams. (Student 2 - Focus group 3)

One student who was confident in using computers said that he learned the concepts through video clips. He read teachers' slides and used business terms to search for relevant clips on YouTube. He felt that those clips with visual information helped him understand the concepts better. Students had their own ways of memorising the concepts. Most of them used key words as clues to review a business definition. One student said that she paraphrased the definitions of business concepts using key terms and her own understanding. Four other students looked for links between the concepts and subject matter and reorganised diagrammatically, as explained by this student:

I often use my notes and a mind map to display the business concepts. Each concept includes some key words supported with relevant examples or explanations. After each chapter, I try to link the concepts together. This has helped me to memorise them more easily. (Student 2 - Focus group 1)

In class, students responded differently to learning activities. While all first-year-students tried to pay attention to what their teachers said, the third-year-students had their own ways of learning. Some of them found it hard to understand the teacher's and their friends' English, so they decided to work on their own, as stated in the quote below:

I am not familiar with the teacher's pronunciation, so I find it hard to understand him. When my friends presented their solutions to assigned case studies, I could not follow their English either. I focused on what I had prepared at home, solved the case by myself and compared with the teacher's slides to know the final answers. (Student 3 - Focus group 1)

Students also made their own choices in managing their language learning. Some students who had little confidence in their English proficiency used both Vietnamese and English in the learning process. They translated concepts, key words or reading texts into Vietnamese to understand everything clearly. Then they worked on memorising English words so that they could do the tests. Some students used both Vietnamese and English textbooks to follow the lectures in class. Other students tried to use English exclusively in their learning, believing that doing so would benefit their learning: 
I try to use as much English as possible in the course so that I can be more confident with business terms in English that I may encounter in books, journals, magazines or news. Most importantly, I can train myself in the ability to think in English, which I find it very helpful for my study and future work. (Student 2 - Focus group 1)

In summary, the students responded to the challenge of learning in their EMI course by regulating their own learning. They invested time in self-study at home to prepare for each class. They used different strategies to understand business concepts such as going over definitions, exploring explanations and looking for relevant examples. The students made choices about using English and/or Vietnamese in their learning.

\subsubsection{Collaborative learning}

All the students mentioned their group activities in class. Students in the first focus group reported working on case studies in groups. Those in the second and third focus groups joined in group discussions to solve problems. Those in the last focus group stated that they explored video cases by collaborating with group mates. Most of these students valued working with others, as indicated below:

I enjoy group work activities in class. The teacher often provides us a case study or shows us a video clip followed by some questions to discuss. We work in groups to find out the answers. We can choose to report our answers with or without slides. If we have good answers, our group will get one bonus for our mid-term paper. (Student 5 - Focus group 4)

Some students found that discussing or working in groups was useful for their learning as they could share their opinions easily with their friends. The students conducted group work in different ways. Some selected a group leader who was responsible for allocating tasks to each member. They discussed how to complete the assignment the most effectively. Students were in charge of their own section and then shared their work with the whole group. Many students who had concerns about their English proficiency believed that they learned better with help and support from their group mates, as evidenced in this quote:

My English is not good, so I often get my group leader to translate for me. With a group assignment, the leader first gets us to read all texts and gather the materials needed, then allocates tasks for each member; finally, we share our understanding together. This helps me feel confident on my part and understand the whole assignment. (Student 4 Focus group 1)

Some students, especially first-years, also complained about how the teachers organised group activities in class. They found they could not complete these properly in the short time allocated, as this student said: 
We have to move into groups as the desks and chairs are fixed. We normally discuss in Vietnamese. However, we have to answer the teacher in English, which is difficult, as we have not translated our answer into English yet. (Student 4 - Focus group 2)

A few students shared unwelcome experiences from collaborating with friends in class. They felt it was unfair when some of their classmates were too lazy to contribute to the shared tasks. Two students who were good at English reported differences in language preference in some groups, which made them feel demotivated to participate in collaborative learning activities, as indicated in the following quote:

Some of my group mates only focused on their section, so they did not contribute or share their opinions to others' parts. In addition, I prefer discussing in English instead of using Vietnamese and then translating again in English, but few members want to do so. (Student 1 - Focus group 3)

These students decided to study on their own to get more out of their learning. When assigned to work in a group, they chose only to complete their own part, which suggests that the group activities did not always lead to collaboration. Most, however, enjoyed working together, sharing opinions and helping each other with difficulties, especially those associated with English. Some students preferred to work individually though when they felt that the teachers did not allow them enough time; their groupmates did not contribute properly; or they could not use English in groups.

In summary, the students expressed their preference for collaborative learning. They valued group activities set by the teachers. They managed group work so that they could share and help each other to complete assigned tasks effectively. However, some students did not engage well in group activities and preferred to work on their own. Those students found it challenging to collaborate with their classmates who were not at the same level of English proficiency.

\subsubsection{Digital competence}

This sub-theme explores how focus group students demonstrated their digital competence in learning economics subjects through English. All participating students felt confident in their ability to use digital technologies. They commonly used laptops and mobile phones for different learning activities and believed that these technologies were indispensable for their learning. (see Figure 8). 


\section{Figure 8}

Students' digital competence demonstrated in their learning

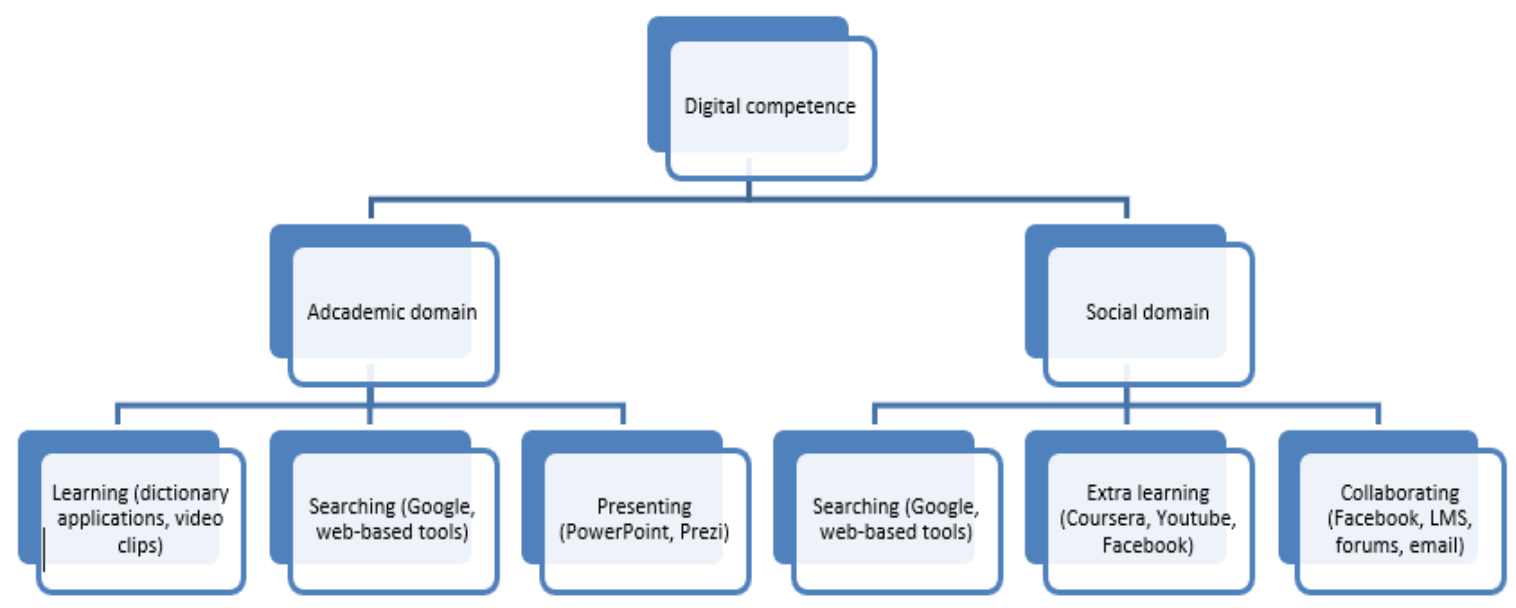

The students' integration of technological equipment into classroom activities was still limited and they mainly used digital technologies to search for and present information, as evidenced in the representative quote:

In class, the teacher sometimes assigns group presentations, so I prefer to use my laptop for presenting as I always use PowerPoint or Prezi to design slides. Slides help me include image, videos and sounds easily. (Student 3 - Focus group 1)

The students said that the teaching tasks did not really require them to use technologies. For example, they did not have to use PowerPoint slides in their presentations. However, those students who presented with slides felt that they performed better using slides as a visual display tool or an information reminder.

Further, all the students were familiar with a number of different search tools on their laptops and mobile phones. With the permission of teachers, they accessed the Internet through their Wi-Fi connected phones to look for information in class. Students in case study four reported that online searches were essential to many of their learning activities in class, as described by one student:

I connect to university Wi-Fi to search for information online. Google is a "Mr. Know-It-All" as I can find anything relevant to my subject. It can provide me with links to different websites or resources. In class, mobile phones are commonly used for searching purposes. (Student 6 - Focus group 4)

Many students valued the teachers' use of video clips in their lectures, reporting that they understood the subject matter more easily when it was presented this way, as explained in this quote: 
Watching clips is more exciting than reading books, listening to the teacher or looking at slides. Clips contain visual information that help us get the ideas more quickly, even in English. The teacher then shows us subtitles as well. (Student 3 - Focus group 4)

Because they were new to the EMI courses, the students shared a common need for English support. They felt that translation was the best way to achieve this. They were familiar with a variety of dictionaries, especially electronic ones, which not only helped them look up meanings of new words but translated reading texts as well, as given in this quote:

I have installed different dictionary applications in my laptop and mobile phone. They are free and easy to use. Some of them can be used off-line, so I can look up words anytime conveniently. I often use Google translation for reading texts or homework instructions. (Student 4 - Focus group 3)

Outside class, the students reported using digital technologies for different purposes. They all spent a great deal of time using digital devices to support their learning.

All the students reported using laptops and mobile phones to search for information. They said that their devices were connected to the Internet most of the time. They used wireless Internet on the university campus, at the university library, at coffee shops and at home. They had data on their phones as well which allowed them to use Google to search whenever they needed extra information or reference materials, as in the example below:

The Internet at university sometimes lags seriously due to too many users. However, I can get Wi-Fi access easily at coffee shops around the university. The connection may be slower at home, but I can use 3G sims or data packages on my mobile phone. (Student 5 - Focus group 2)

The students had access to a wide range of resources online, so they needed to process the online information to select what was relevant to their learning. They were aware of strategies to look for information from different academic websites, electronic journals, forums or social networks like YouTube or Facebook, as indicated in this quote:

Using key words in the course books or the teacher's slides is the most effective way when searching for something. I often start with reading Q\&A sections in a forum or Facebook groups. I also use the references provided in the course book or further reading materials from the teachers. (Student 3 - Focus group 4)

Many students used digital technologies to exchange information and interact with others. All of them had class Facebook accounts through which they updated information about their subjects and courses. They spoke about different groups on Facebook where they sought help or support in their learning, as given by this student:

My class has a common group on Facebook to update information related to the course. I am a member of a student association group where I can chat with senior students or 
those from different classes. I also joined a marketing group and a business administration group whose members are not only students but also employers, researchers, lecturers or experts in the field. (Student 5 - Focus group 1)

Three students said that digital technologies helped them learn both English and content by themselves. For example, they enrolled in complementary online courses in different areas: I am taking one course in digital marketing on Facebook and another course in Finance on Coursera, which is a website providing online courses in various areas. These courses help me learn subjects related to economics, which is very useful for my major. (Student 1 - Focus group 3)

Two students stated that they used applications installed on their laptops and mobile phones to manage their learning, as this student explained:

I always transfer my notes into digital formats that I can store in Dropbox and Google Drive. I use a mind map application to summarise each lesson and arrange these lessons into different categories. I can retrieve them easily from my laptop and mobile phone when I need to review for exams. (Student 4 - Focus group 1)

All students believed that online learning or e-learning was helpful for them. They could contact the teachers, receive updates about the subject and instant feedback on assignments, review the lectures with quizzes, and receive extra links or materials from the teachers. However, they all felt that the LMS used at the university was still limited, as stated in this quote:

The teacher requires us to use the LMS, mainly for receiving and submitting assignments, getting notices and reference materials. However, the system runs slowly. It does not have a notification function, so I have to check it every day and feel disappointed when having no updates. I think it is boring and time-consuming, too. (Student 6 - Focus group 2)

In summary, all students had high confidence in using digital technologies in their learning and commonly used digital support both in class and out of class to support their learning. They reported different active uses of technology including searching for resources, designing and presenting information, communicating with other people and taking online courses. These uses show their competence in deploying different types of technology for conducting various modes of learning.

\subsubsection{Personalised learning network}

This sub-theme explores how the students in the focus groups created their own learning networks through collaborating with people around them. All the students participated in different learning networks outside class and enjoyed not only exchanging opinions with their peers but also seeking advice from experienced mentors or experts. They actively engaged with peers in their daily life and used digital technologies to join virtual communities. Social 
networking, especially Facebook, was a preferred channel for students to connect with others. All the students joined their class groups on Facebook to communicate with each other and stay up to date on any information related to their learning, as evidenced in this quote:

We all use Facebook to chat and exchange information. The class monitor often posts announcements from the teachers. Everyone can raise questions, share opinions and discuss the assignments. We do not add our teacher into this group so that we can speak comfortably. (Student 1 - Focus group 4)

Some students had different reasons for joining different online groups. They wanted to seek advice from senior students who had finished the same courses or look for videos or lectures related to the subjects they were learning. Other students wanted to interact with experts or members in groups that specialised in different areas in economics. They valued the interactions with these groups:

It is easier to talk to others in virtual social groups. In the groups that I joined, everyone is willing to share their opinions and experience. I learn a lot from them. Some people even contact me privately to offer help or extra information. (Student 3 - Focus group 1 )

While Facebook groups were popularly used among students themselves, the LMS and email were used for interactions between the teachers and students. The students also asked for help from different teachers in their school, especially their academic consultants.

Although many students found it valuable to engage in social interaction in virtual communities, a few students preferred face-to-face communication with other people. Some students formed their own group to work on the teachers' assignments. They then felt connected to each other and were happy to collaborate as peers. Some other students participated in student-run academic clubs where they had frequent meetings and events to work with others, as stated below:

My economics club meet every two weeks to discuss different topics. We sometimes organise quiz shows, or competitions related to our subjects. The members in the club always share with each other their experience and useful materials. (Student 4, Focus group 3)

One student, however, stated that she felt overwhelmed with too much information from different groups. Worried that information could become confusing or contradictory, she preferred to restrict social interaction to the network from her part-time job:

I like communicating with my workmates and my supervisor in my part-time job. They often give me advice related to my subject, which is often practical and applicable to what I am dealing with. I can also improve my communication skills and expand my network. (Student 5, Focus group 1) 
In summary, the students benefited from social interaction in their personal learning networks and created connections with different people to form their own networks of learning (see Figure 9). Many of them enjoyed participating in various groups on Facebook. Those groups helped them connect with their classmates, friends in different universities or courses, senior students in their schools, or even businesspeople, experts and lecturers in their field of study. Some students interacted with their lecturers and academic consultants through the LMS and email system. Despite being familiar with technologies, some students valued face-to-face communication more. They worked in their groups of peers to understand and complete the teachers' assignments. The students also joined student-run academic clubs where they could meet and interact with other students. A few students with part-time jobs enjoyed social interaction with their workmates and especially their supervisors who gave helpful advice related to their study.

\section{Figure 9}

\section{Students' personalised learning network}

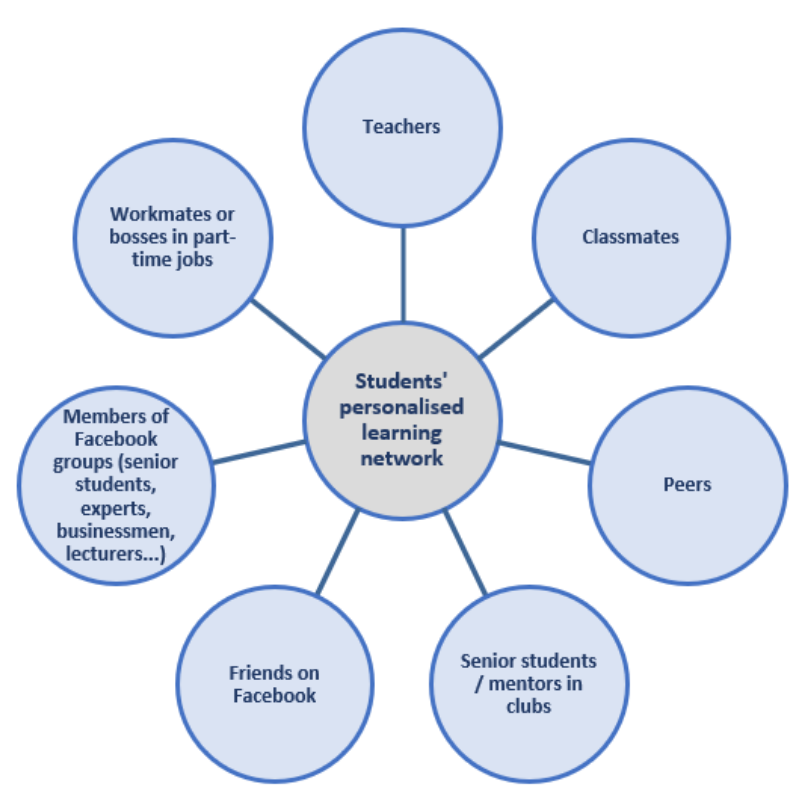

\subsubsection{Summary}

This theme explores how students developed autonomy in the learning process when participating in the EMI programmes. Three aspects of autonomy were explored as sub-themes including student agency, student digital competence and student personalised learning networks. In terms of agency, students showed their ability to control their own learning and demonstrate their capability for independent and collaborative learning. Students devised strategies that helped them overcome difficulties in the new learning context and made choices to collaborate with others outside class to enhance their learning. Regarding digital competence, 
students used digital technologies effectively to support their learning. They autonomously integrated technologies into different learning activities in both academic and social domains. Finally, students took an active role in personalising their learning network. They connected with different people in both virtual communities and real life to support their learning. They believed that social interaction in their networks helped them significantly in their learning. This appears to be unique to EMI programmes in which students have to acquire content knowledge and develop English proficiency without sufficient support from their teachers and the institution.

\subsection{Summary of key findings in response to research questions}

The cross-case findings analysed different themes capturing the teachers and students' experiences in the courses. These themes included: An expectation - reality gap; Pedagogical decisions; Integration of content, English and digital technologies; and Developing autonomous learners. This section summarises significant points from the findings in response to the four research sub-questions to answer the research question: How are digital technologies used in an EMI context in Vietnamese higher education? (see Chapter 3)

\subsubsection{How do teachers and students use digital technologies in an EMI environment?}

The teachers used technologies across different aspects of teaching. They curated and developed resources including reference materials and multimedia resources for the preparation and delivery of content knowledge to the students. They presented subject matter using digital tools and supported students' understanding of content knowledge by creating resources such as graphical representations, video clips and online quizzes to illustrate economics concepts and review previous lessons. The teachers specifically supported students' English learning by designing slides and quizzes for students to revise and consolidate key vocabulary. Other activities included organising classes through the use of cloud storage and an LMS for uploading materials or communicating with the students, as well as the integration of video cases for engaging students in group discussions. To enact these activities, the teachers used a range of digital technologies including search engines (Google, Wikipedia), presentation tools (PowerPoint, Prezi), organisation tools (Google drive, Dropbox), social media (YouTube), and the LMS. These

findings show that the teachers deployed technology in changing their practice to address different challenges in the context of EMI.

The students used digital technologies for learning in different ways. They demonstrated competence in using technologies including personal computers, laptops, and mobile equipment such as smartphones and tablets that were used to access the Internet, web-based tools and applications for academic practices. They tried to improve their understanding of subject matter 
by accessing multiple resources. They looked for reference materials using search engines, downloaded and uploaded information/resources through the LMS, and organised content knowledge with cloud applications. Those uses appear to serve for the logistics of their study. Moreover, the students dealt with difficulties in studying through English by managing to improve their general English proficiency. They learned and remembered key words using mind-maps and dictionary applications, improved listening skills by watching online lectures in English, and developed their reading skills by accessing English medium materials or courses. Finally, the students also expanded their learning by creating personal learning networks and using online learning platforms. They took online courses on websites and joined virtual communities using social networks such as Facebook. These ways of using technologies allowed them to interact and collaborate with others to support their learning. This finding indicates that the students were proactive in their use of digital technologies for learning in the EMI environment.

\subsubsection{How do teachers perceive their students' learning to have developed through digital technologies in an EMI environment?}

The teachers believed that their students benefited from the use of digital technologies in their EMI classes. They perceived that the students gained content knowledge through multimedia digital resources such as graphs, charts, or video clips used in lectures. They felt that students' engagement and motivation increased through the use of online quizzes and video case studies and that students developed their familiarity with English business terms and concepts. They thought that their adoption of technologies into teaching activities such as vocabulary quizzes, visual information, or clips with English subtitles helped students learn, understand, and memorise words effectively. The teachers therefore perceived positive impacts of digital technologies on student learning in the EMI environment.

5.5.3 How do students perceive their learning to have developed through digital technologies in the EMI environment?

The students believed that using digital technologies enhanced their understanding of content knowledge and development of English proficiency. Access to digital resources and connections with other people in their learning networks provided them with support in learning subject matter in English. They were able to develop their vocabulary and use a range of applications and tools to learn business terms. The students developed their English skills through watching online lectures or YouTube clips, reading materials in English, and interacting with others in social groups or forums. In sum, the students perceived that digital technologies played an integral role in their learning of subject matter and English in the EMI environment. 


\subsection{Chapter summary}

The findings show some gaps between what the teachers and students expected and what they experienced in the EMI courses. The teachers expected to receive training on EMI pedagogies instead of English support. This meant that they were unaware of best practice for teaching in a

CLIL environment. Furthermore, they were not all able undergo the training because there were too few courses offered. The teachers were unable to access good quality teaching materials and digital resources. They also complained about the students' English proficiency, which was not only varied but also limited. The students in the study realised that their preparedness for participating in the EMI courses was insufficient as they struggled to understand content presented in English, and with the overwhelming content load. In particular, they found it difficult to maintain the intense concentration required during a long lecture delivered in English. The students also felt disappointed that they could not to develop content knowledge and English competence simultaneously and attributed this to the teachers' limited English proficiency.

As for the teachers, they made pedagogical decisions intended to support students' learning in the new context of EMI courses. Firstly, the teachers made new content accessible to learners by providing authentic contexts and including practical examples related to the Vietnamese context. These examples were relevant to students' daily lives and connected to their background knowledge. Secondly, the teachers differentiated their teaching to match students' varied background knowledge and competence. Specifically, they selected appropriate content, used simple English to explain the subject matter, and presented content with visual information to assist students' understanding. Thirdly, the teachers facilitated students' analytical thinking and problem-based learning. They used a range of questions to initiate students' thinking processes, used graphs, charts, and tables to engage them in analysis and involved them in case studies to emphasise the need for problem solving. Finally, the teachers engaged students in collaborative and interactive learning by including group work discussions and presentations and asking questions designed to enhance interactions with and among students. They also interacted with students outside class.

The use of English as a medium of instruction caused some difficulties for all the teachers. Although the teachers adjusted their pedagogy in response to the integration of English and digital technologies in teaching content knowledge, they found it hard to convey subject matter in English and had concerns about their own English competence. Thus, they simplified the language used to give explanations and avoided expanding the lectures to cover content in depth. They used code-switching, translation and translanguaging strategies to ensure students' 
comprehension of the subject matter. Despite limited professional learning about how to teach in English and neglecting to include any English learning objectives in the lectures, they tried to engage students in interactive activities like questions and answers, and discussions and helped them learn business concepts and key terms effectively. Some of those activities had the potential to enhance their students' English competence but the teachers did not know how to apply strategies to purposefully maximise language learning.

In terms of technology integration, the teachers did this in different ways. To prepare lectures, they accessed the Internet to search for information using Google, downloaded materials like articles or video clips from websites or YouTube, and created teaching resources such as graphs, charts, and tables. In class, they used slides to present the lectures and show visual information, conducted online quizzes for consolidation and had students do computer-based exam papers. The teachers also interacted with students through different channels such as email, the LMS, their personal websites and Facebook, providing students with further reference materials using these digital technologies as well.

The students in the study demonstrated their autonomy to adapt to the new learning context. They controlled and personalised their own learning by exercising agency, digital competence and personalised learning networks. Agency can be seen in their use of different strategies to learn the subject matter through English both independently and collaboratively. They invested time in self-study, learned business concepts in different ways, and used English and Vietnamese strategically to effectively understand the subject matter. The students also manipulated group work activities in class. As for digital competence, the students used digital technologies in both the academic and social domains. At university, they mainly deployed technologies to search for information, present information, and learn both English terms and subject matter. Outside class time, at home or with peers, the students collaborated with others and undertook extra learning online. Finally, each student in the study had their personalised learning network. They created connections with different people to seek assistance in their learning. They benefited from these diverse forms of collaboration and social interaction across their learning networks. 


\section{Chapter 6. Discussion}

This chapter discusses significant findings in connection with the ROAD-MAPPING framework (Dafouz \& Smit, 2020) (see Chapter 2) designed as a conceptual framework to underpin the investigation of English-Medium Education in Multilingual University Settings (EMEMUS). This framework therefore fits my study of academic programmes taught through English at a Vietnamese university. Through the lens of ROAD-MAPPING, I begin by addressing the multifaceted nature of EMI programmes in the Vietnamese HE context before turning to the teaching and learning of EMI courses in the digital age in the changing context of Vietnamese society.

\subsection{The implementation of EMI programmes in the Vietnamese context}

English-medium education (EME) in HE occurs in a wide range of contexts across the globe. Each context offers EME programmes with different characteristics and specificities responding to the influence of factors such as policies, academic disciplines, societal roles of English and the relationships between international and local students and their lecturers. In Vietnam, English as a medium of instruction has been adopted as a "proactive approach to internationalisation of higher education" for the last two decades (Tran \& Nguyen, 2018). However, there is little empirical evidence on how EMI is implemented in Vietnam's HE sector (Dang \& Moskovsky, 2019). The first part of this chapter therefore aims to discuss the contextual features specific to EMI programmes in Vietnam using the ROAD-MAPPING framework as a means to "capture and analyse the dynamic, multi-layered and diverse nature of EMEMUS" (Dafouz \& Smit, 2020, p. 59). This holistic framework conceptualises six intersecting, but independent dimensions including: Roles of English, Academic Disciplines, (language) Management, Agents, Practices and Processes, Internationalisation and Glocalisation. In the ROAD-MAPPING framework (see Figure 10), Discourses is placed in the centre as the intersecting point of all six dimensions (Dafouz \& Smit, 2016). Discourses are social practices including policy documents, notes, interviews, discussions, and other oral and written texts. In my study, the main point of access is the discourses of teachers and students participating in the EMI programmes at a Vietnamese university. In other words, findings discussed in this chapter are EMI experiences of the teachers and students collected from interviews, focus group discussions, and classroom observations. 


\section{Figure 10}

The ROAD-MAPPING framework for EMEMUS (Dafouz \& Smit, 2016, p. 404)

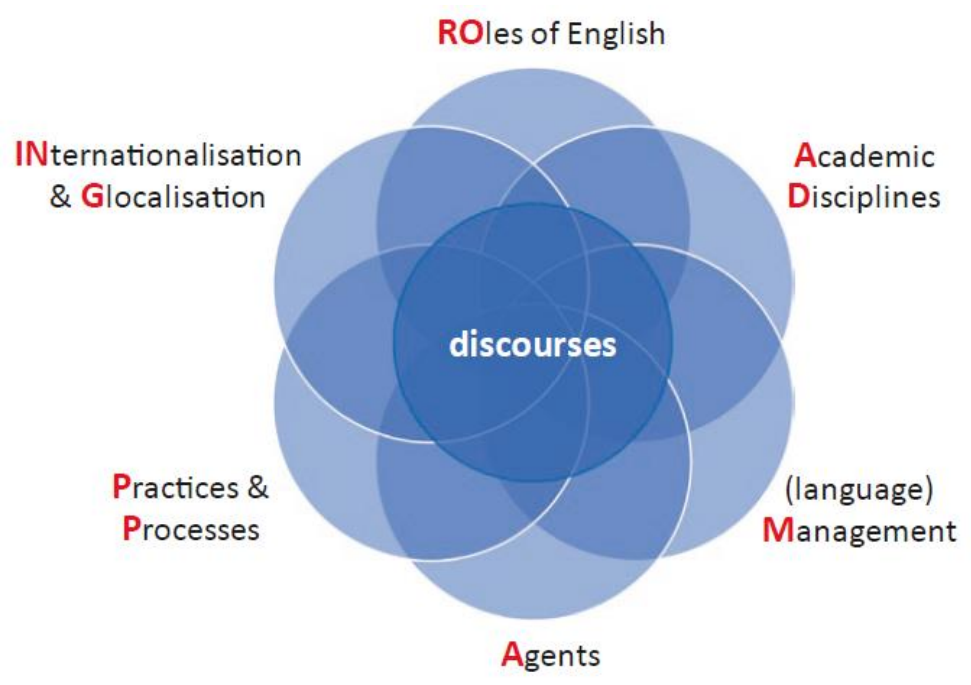

\subsubsection{Roles of English}

English has a privileged status as a global language in academia and HE (Dafouz \& Smit, 2020). In EMI settings, English performs a number of functions. It is the language of teaching and learning, a means of regulating student enrolment or managing staff recruitment, and/or a lingua franca for staff and students (Baker \& Hüttner, 2017). In Vietnam, despite the increased use of English as a result of globalisation and economic growth, English as a foreign language "is used in relatively limited domains such as in foreign trade and tourism" (Hashim \& Low, 2014, p. 424). In the education system, while Vietnamese is still the dominant language of instruction, English is compulsory as a standalone subject. English is taught and learnt distinctly from other subjects in the curriculum. However, the shift in the instructional language from Vietnamese to English has marked changes in the roles of English in HE. English can now be seen both as an instrument or by-product of content teaching and learning and used for monolingual or bilingual instruction.

\subsubsection{English: an instrument and a by-product}

All the participants viewed English as an instrument for academic purposes and as something that provided professional opportunities. In EMI courses, English was a tool for teachers to deliver content and for students to engage in learning content. Specifically, in the focus university, the teachers and students advocated the use of English as they believed that as an international language, especially in the business world, English was appropriate for teaching and learning economics subjects. This perception is reinforced by many studies that have shown 
the dominance of the English language in the field of business education (Wilkinson, 2011). The global status of English also offered teachers and students opportunities to integrate into the world outside Vietnam. This was one of the salient drivers motivating them to participate in EMI programmes and both students and teachers believed that teaching and learning in English would enhance their professional mobility. The students especially felt that learning in English would afford them opportunities to compete in the global job market. These perceptions and aspirations are in accordance with the national Vietnamese HE policy whereby English is portrayed/conceptualised as a global language and its use is encouraged to facilitate the production and transmission of scientific knowledge with perceived benefits for teachers' and students' professional and academic advancement (Dang \& Moskovsky, 2019).

The participants expected that proficiency in English would be a by-product of learning business through the EMI programmes. Even though the teachers and students stated that they understood English to be one of the targets of the courses, they did not set any objectives that would monitor English teaching and learning. This might have arisen from the common belief that students develop their English proficiency automatically through exposure to English in the courses (Lasagabaster, 2017; Nguyen, 2018). This belief is consistent with Krashen's (1985) input hypothesis that theorises that students develop language competence unconsciously when exposed to language input at an appropriate level of difficulty (Coşgun \& Hasırcl, 2017). Indeed, participants seemed to expect English language development to follow as a natural outcome of the process of learning content through EMI courses (Uchihara \& Harada, 2018) and this may explain why they did not explicitly target English skills in their classes (Nguyen, 2018). However, research suggests that "merely teaching a course previously taught in the national language in English alone is unlikely to achieve significant linguistic benefits" (Chapple, 2015, p. 8). Under some circumstances, both students' content comprehension and their English improvement may even be compromised by EMI (Lei \& Hu, 2014). In other words, contrary to the beliefs of the participants in this study, studies have found that content lecturers need to explicitly provide students with language support if they are to develop English skills in EMI programmes (Cots, 2013).

\subsubsection{English: monolingualism and bilingualism}

Using English as the medium of instruction impacts the use of the first language in the curriculum (Dafouz \& Smit, 2020). As mentioned in this chapter, Vietnamese is still the dominant language of instruction in Vietnam. EMI programmes are not implemented in all parts of the tertiary curriculum and in this study participants learnt some economics majors in English, and 
others in Vietnamese such as Philosophy, Advanced Mathematics, and Political Economics. This inconsistency across the institution created confusion for both the teachers and students, even in EMI classes. They reported that English was not exclusively the language of instruction in EMI classes. Since there were no specific guidelines on how much English should be used in class and Vietnamese was the shared language, few teachers adhered to the English-only policy of in their classes. While some teacher participants tried to solely use English in class, others consciously and flexibly switched their language of instruction between English and Vietnamese. The teachers' practice of using English for content delivery but Vietnamese for assessments may have compromised their students' English development. This phenomenon was reported in Symon and Weinberg's (2013) study: "if the only exposure to English is during lectures, and if assignments and examinations can be submitted in their first language, then improvement in English will be minimal" (p. 23). Meanwhile, there was also a concern among the students about the impact of the language used for communication in classes. They worried how this might affect their future employability. The exclusive use of English might ultimately equip them with English to compete in a global job market but nonetheless restrict their ability to communicate subject matter in Vietnamese in a local organisation. Generally, while the implementation of EMI aimed to promote the use of English as the medium of instruction, the participants did not feel that this was achieved while Vietnamese was still widely used in teaching the curriculum and there were no clear guidelines for language use in the EMI setting.

\subsubsection{Academic disciplines}

According to Loewenberg-Ball et al. (2008), teachers of an academic discipline need a knowledge base in which "knowledge of content, knowledge of teaching, and knowledge of students interact with teachers' specialised knowledge for teaching a particular content area" ( $p$. 3). In Vietnam, an EMI priority has been seen in disciplines like information technology, business administration, banking and finance, accounting (hard-applied), and tourism (soft-applied), which have the potential to benefit Vietnam's socio-economic development (Dang \& Moskovsky, 2019). The tendency to import Western academic programmes for these disciplines in Vietnamese universities has created pressure for teachers to make curricular adaptations and pedagogical changes. The teachers in my study, who were experienced in teaching their subjects in Vietnamese, believed that they had appropriate pedagogical skills for their content area but had not yet learnt to engage the additional skills for teaching in an additional language.

Teaching and learning an academic discipline in a foreign language has an additional dimension that relates to "the different teaching and learning genres, curricular design, and 
assessment methods used in the academic setting" (Dafouz, 2018, p. 175). Each academic discipline has its linguistic conventions for making meaning. These discipline-specific linguistic conventions are reflected in specific genres / discourse types and highly specialised terminology (Dafouz \& Smit, 2016). Teachers need to recognise and understand the features of their disciplinary discourse to be able to "communicate the subject matter in flexible and multifaceted ways" (Turkan et al., 2014, p. 8). These disciplinary features are also reflected in the preferred pedagogies of particular disciplines. For example, the field of business education is generally considered in the quadrant of a soft-applied discipline that strongly focuses on practice and coconstruction of knowledge in the classroom (Gleeson, 2015). Effective EMI/CLIL teachers are those who can help students learn disciplinary knowledge, raise their awareness of the characteristics of academic language, and facilitate the academic language of the disciplines as well (Turkan et al., 2014). A body of recent literature in Systemic Functional Linguistics differentiates language features specific to communicating in science, mathematics, English language arts, and social studies (Brisk, 2014; Schleppegrell, 2020). This suggests that subject teachers need to identify the linguistic features of their subject so that they can make these explicit to their students. However, the subject lecturers in my study did not appear to be aware of the language forms and genres typical of their subject, or suitable teaching strategies that might enhance students' learning of content in English.

From the students' perspective, learning disciplinary knowledge required them not only to understand the concepts but also to apply and communicate the knowledge in practice (Komori-Glatz, 2017). These EMI students expected to learn subject matter and also to learn how to convey meaning in the content area in English. In other words, they wanted to read, write, listen, speak, and even think in the language of the discipline (Turkan et al., 2014). This explains why some students in the study favoured learning in English only as they wanted to learn how to process information in English. Even though there were no specific objectives for learning English, the students implicitly appreciated that there were specific language demands in the content areas. This raises implications for content lecturers in Vietnamese contexts who have not been prepared to teach language as well as their subject (Nguyen et al., 2016).

\subsubsection{Language management}

Results from all the interviews showed participant awareness of the university language policy promoting the use of English. The participants were much less aware, however, of how this policy should be enacted. The university used students' English language proficiency as a gatekeeper for entry to and exit from academic programmes. This aligns with the Ministry of 
Education and Training's (MOET, 2014) requirement that students entering EMI-based programmes should be assessed according to the six levels of language proficiency from the Common European Framework of Reference for Languages (CEFR) (Nguyen, 2018). However, this policy is applied inconsistently as students' results in English tests namely the Test of English for International Communication (TOEIC), the Test of English as a Foreign Language (TOELF), and the International English Language Testing System (IELTS) are all accepted by Vietnamese universities. The use of TOEIC, TOEFL or IELTS to place students has always been controversial (Choi \& Park, 2013). The validity of these tests is also questionable in that some tests appear to focus on testtaking strategies rather than purposeful English proficiency (Shim \& Baik, 2003), and may assess against a notional native speaker norm instead of taking into account other varieties of English (Lowenberg, 2002). For example, despite its increasing popularity as a university entrance assessment tool in many Asian universities including Vietnam, the TOEIC test still uses a multiple choice format to assess listening and reading skills, and speaking and writing skills tests are optional (Hsieh, 2017). Further, little significant correlation has been found between students' IELTS scores and their academic performance (Feast, 2002). These findings are pertinent in light of the particular research context under investigation in that placement tests were designed in the format of TOEIC tests and students also had the option to submit IELTS or TOEFL scores to establish their English proficiency. Because these tests do not assess the English proficiency of the students in the discipline of business studies, the use of such tests did not guarantee that students had sufficient disciplinary literacy in English to manage learning in EMI.

\subsubsection{Agents (Actors)}

Teachers and students are the two salient social players engaged in EMEMUS (Dafouz \& Smit, 2020). In other words, they are the agents who play a significant role in shaping EMI (Bradford \& Brown, 2018b). In Vietnam, teachers are viewed as the actors who bring their expertise to the implementation of EMI; and students are those not only with an interest but also power to influence the process of EMI development (Dang \& Moskovsky, 2019). In this study, both teachers and students as key agents participated in EMI according to their beliefs and knowledge.

\subsubsection{The teachers}

The teachers involved made deliberate pedagogical decisions in response to the new English medium of instruction in their economics courses. These decisions reflected their beliefs and knowledge about teaching within their academic disciplines. 


\section{Teacher beliefs}

As discussed in Chapter 2, teachers' beliefs play a significant role in predicting, reflecting, and determining their actual practices (Kim et al., 2013; Wilkins, 2008). Study findings reveal significant impacts of the subject teachers' disciplinary beliefs and self-efficacy on their teaching activities.

\section{Disciplinary beliefs.}

Teachers' disciplinary beliefs appear to be reflected in their teaching approaches in the new context of EMI. In my study, the economics teachers believed that teaching disciplinary subject matter was the priority. As content teachers, the teacher participants believed that the goals of their courses were to enhance students' comprehension of content knowledge. Such disciplinary thinking directed their intentions to focus on designing activities for disciplinary content teaching rather than language teaching. As a result, only two participants thought that they needed to make significant changes to what and how they were teaching. This finding is similar to what Dafouz et al. (2016) found in their study on university teachers' beliefs about language and content in EMEMUS. They reported that the lecturers refused to change because they "identify their teaching focus as remaining on content exclusively" (p. 137). The subject teachers did not include any English teaching objectives in their lectures and neither did they pay attention to students' English use in class. This result aligns with other EMI research where subject teachers assume that they are not responsible for the teaching of English (Ali, 2013), and that they do not need to support students' English (Dearden \& Macaro, 2016).

\section{Self-efficacy}

Teachers' sense of self-efficacy seems to shape their teaching practices in the EMI environment. The teachers in my study demonstrated high levels of self-efficacy in teaching their subjects and transferred this confidence to teaching their subject through English. All of them worked hard in looking for appropriate strategies and approaches to enhance students' comprehension, engage them in interaction, and develop their autonomy. Despite admitting the challenges in teaching the EMI courses, they all expressed great confidence in their capability to undertake and execute this teaching responsibility. This can be explained by the fact that all of them were experienced in teaching their subjects in Vietnamese, so they were confident about their strong content knowledge. In this case, the teachers' self-efficacy was consistent with their disciplinary ways of thinking, which strengthened their belief that they could teach content knowledge even through another language. Interestingly, such beliefs seemed to result in different pedagogical decisions. For example, two teachers conducted their lectures in similar 
formats to those they used when teaching in Vietnamese. This does not mean that they tried to avoid complicated tasks, but they believed that the subject matter was equally new and difficult for students in both Vietnamese and English, so it was not necessary to make accommodations to their tried and true teaching approaches. This result is consistent with Dafouz et al. (2016) and also reflects a common belief in EMI programmes that teaching in English is merely translating lectures from students' mother tongue into English (Chapple, 2015). In general, the teachers' selfefficacy is likely to reinforce their beliefs in their disciplinary teaching but might hinder them in changing their teaching beliefs and practices to accommodate teaching and learning appropriate to EMI.

\section{Teacher knowledge}

In line with teachers' beliefs, their knowledge exerts certain impacts on their instructional practices. In the context of EMI, subject teachers appear to make pedagogical decisions based on their content and pedagogical content knowledge.

\section{Content knowledge}

Teaching EMI courses requires teachers to possess knowledge not only about subject matter in their discipline, but also about English as a subject. This knowledge has the potential to complement students' comprehension of content knowledge and facilitate their development of English competence. In other words, in addition to strong background knowledge of the subject matter, teachers need to know how English works as a system in order to explore how to teach different linguistic elements (Banegas, 2020). This requires teachers to understand the language registers needed, be able to identify language features used in disciplinary discourses, and be able to "draw on meaningful metalanguage to raise [students'] consciousness about the ways English is used in the texts they read and write" (Schleppegrell, 2020, p. 17). In keeping with their established roles as content professors, the teachers in my study demonstrated strong disciplinary knowledge, but knew little about English as a subject. What mattered to them was how English was used to explain business concepts, and so they guided the students to focus on technical vocabulary and think critically to comprehend these concepts thoroughly. Having sound content knowledge and experience in teaching, the teachers therefore believed that all they needed a good command of English to be successful in conducting EMI classes. This appears to be a common belief held by many stakeholders in EMI all over the world and has led to a strong focus on general English proficiency in many countries including Vietnam. However, a more salient issue related to teacher knowledge in EMI programmes might be how content teachers can learn to perform the job of language teacher to help students learn subject matter through English. 


\section{Pedagogical content knowledge}

Although the teachers displayed confidence in their pedagogical content knowledge, their teaching practices in EMI programmes seemed to be restricted to content teaching. They believed that their strong disciplinary knowledge background and teaching experience provided them with pedagogical knowledge to teach the subject effectively in English. Their beliefs were seated in pedagogical content knowledge specific to their subject, which led to subject-specific teaching practices. For instance, when designing activities, they wanted to make new content accessible to students, differentiate teaching instruction to meet students' needs, and facilitate students' analytical thinking and problem-based learning, as well as engaging students in collaborative and interactive learning. However, the teachers appeared unaware of the importance of pedagogical content knowledge specific to teaching English and teaching through English, which meant they were unable to help students improve their English proficiency. The teachers did not realise that they needed to equip themselves with the Disciplinary Linguistic Knowledge to know about the academic discourse of a discipline so that they could maximise students' access to content and ability to communicate the language of a particular discipline (Turkan et al., 2014). In short, the participating teachers did not demonstrate an awareness of the difference between teaching content and teaching content through English. Instead, they believed that EMI required only a change in the language of instruction.

\subsubsection{The students}

The students are the other key actors participating in the EMI programmes (Dang \& Moskovsky, 2019). In this study, the students worked hard searching for effective learning strategies to achieve the course objectives, and to help them adapt to the new context of learning through English. The findings showed that they demonstrated high levels of autonomy by personalising their own learning, demonstrating digital competence in searching for resources, and creating personalised learning networks. This autonomy reflects positive self-efficacy and digital competence.

\section{Self-efficacy}

Study findings provides evidence that students' self-efficacy potentially benefit their learning. Students with high self-efficacy are able to engage cognitively, behaviourally, and motivationally in their learning (Linnenbrink \& Pintrich, 2003). Despite the novelty of the EMI context, the focus group participants appeared not only motivated but also fully engaged in the courses. Their belief in the future benefits afforded by EMI boosted and sustained their motivation to persevere. Although they admitted feeling concerned about learning subject 
matter through a foreign language, they worked hard to achieve their goals and gain good results. Some students with low levels of English were still willing to take the EMI courses as they were confident in their capability to complete the academic tasks and assignments. They reported that learning through English encouraged them to be more responsible, hard-working, and focused in their study. Such a high sense of self-efficacy appeared to be at the foundation of students' motivation and encouraged them to proactively engage in learning.

Self-efficacy also appeared to influence students' use of learning strategies. Students possessing strong self-efficacy have been found to be persistent in coping with obstacles and distraction, as well as being likely to purposefully employ particular strategies (Caprara et al., 2008). The students in this study had to look for appropriate learning strategies to understand content knowledge through English. They not only had to learn a great number of business concepts and principles, but also needed to learn these concepts in English. They had to adjust their learning habits and search for effective ways to overcome these challenges. For instance, many students reread the texts at home, looked up the meanings of key words, linked important points together, and memorised them in English, or translated them into Vietnamese to understand them more thoroughly. This self-efficacy motivated the students to regulate their learning, facilitated the selection and use of learning strategies, and engaged them in the learning process.

\section{Digital competence}

Digital competence has been found to be among the key intellectual resources supporting autonomous learning. Digital competence is defined as "the skills, knowledge, and attitudes that make learners able to use digital media for participation, work, and problem solving, independently and in collaboration with others in a critical, responsible, and creative manner" (Hatlevik et al., 2015, p. 124). According to this definition, digital competence is an important component of students' learning. The students participating in EMI courses had to contend with new content learning, limited English proficiency, and lack of guidance and support. Fortunately, they had access to multiple resources on the Internet, which enabled them to utilise their technical skills in their learning both in social and academic domains. These students played an innovative role in the learning process through actively regulating their own learning. For instance, they discovered how to create their own learning networks with the assistance of digital technologies so that they could interact and collaborate with others, demonstrating high levels of digital competence in their learning practices. These digital skills appeared to be strong enablers for students to proactively manage their learning and confidently deal with the challenge of EMI. 
This independent use of digital technologies emerges as a salient factor facilitating students' autonomy in learning.

\subsubsection{Practices and processes}

According to the ROAD-MAPPING framework, the dimension of Practices and Processes refers to "the teaching and learning activities that construct and are constructed by specific EMEMUS realities" (Dafouz et al., 2016, p. 407). These activities reflect different types of classroom practices facilitating the joint development and construction of knowledge. Dafouz and Smit (2016) have distinguished three key relevant practices in EMI settings. The first type of practice reveals the "localised process to develop a shared repertoire" (p. 407) in which English is used not only as a disciplinary and educational language but also as a lingua franca for academic and social communicative purposes (p. 407). The second type of practice emphasises teachers' beliefs about how students learn and how to best support students. The final type focuses on the development of academic literacy skills affected by the teachers' ability to integrate content and language teaching, or the relationship between subject specialists and language. To exercise these types of practices, EMI teachers are required to make significant changes to their teaching approaches. However, changes in pedagogical practices appear to challenge most teachers as they work within the many constraints of the educational system, academic culture, and students' background. The following section discusses the teacher participants' experience of shifting their pedagogical approaches to accommodate the EMI context which required them to balance teacher-centred and student-centred approaches, content or quality interaction, and make the decision whether to use English-only instruction or integrate Vietnamese and English in their classes.

\subsubsection{Teacher-centred or student-centred approach}

In Vietnam, student-centred approaches were advocated in the Higher Education Reform Agenda by the Ministry of Education and Training in 2005 (Tran et al., 2018). Recent decades have witnessed increasing student-centred approaches in HE in many Asian countries like China, Malaysia, Singapore, Hong Kong, Taiwan, Korea and Vietnam (Pham, 2011). In Vietnam, a further objective for implementing student-centred learning approaches in tertiary institutions is to create a stimulating learning environment for students' construction of knowledge and skills (Tran \& Lewis, 2012).

Academics in Vietnamese universities, especially those involved in EMI programmes, have been working on developing student-centred teaching. This is reflected in lecturers' pedagogical practices which employ a range of active teaching strategies to encourage students to take an 
active role in learning. In my study, the teachers deliberately changed their teaching practices to engage students in classroom activities. For instance, Vincent and Nancy used video case studies as the basis for group work and problem-solving activities. In such activities, the students were required to take responsibility for their learning by analysing the tasks, searching for relevant information, collaborating with others in groups, and building ideas. The teachers guided their students' learning by creating an interactive and collaborative environment in which they adjusted their own use of language to accommodate the students' different levels of English as well. Therefore, the teachers appeared see themselves in the new role of facilitators supporting students to develop analytical thinking and problem-solving skills by differentiating their instruction rather than transmitting knowledge to students in the traditional way. This finding is consistent with Wilkinson's (2011) observation that "student-centred approaches such as these enable students to actively develop both content and language knowledge, skills and abilities in EMI environments" (p. 11). Even though the teachers might not have been aware of the characteristics of a student-centred approach, their employment of constructivist pedagogical strategies appeared to support students' development of autonomy in learning, a key construct of student-centred learning (Lee, 2017).

Among those strategies used to help EMI students construct knowledge, scaffolding appears to be the most common teaching practice. In the new EMI context at the university, students' limited background knowledge of either business or English required teachers to scaffold them with "explicit structure[s] to make sense of content, make informed decisions, monitor their progress, and adapt to emergent challenges" (Lee \& Hannafin, 2016, p. 719). To do this, the teachers in my study (who prioritised students' understanding of subject matter) employed a range of comprehension and problem-solving scaffolding strategies to support students. Typically, the teachers in EMI courses used amplification to scaffold students' learning especially with the assistance of multiple digital resources (Smit et al., 2017). For example, all the teachers reported using simple language to give full explanations to students, not simplifying the content of the lessons but sometimes reducing the amount of subject matter in their classes. They created bridges for students to access new concepts by activating their prior knowledge. They also found multimodal materials and created a rich experiential context to trigger students' curiosity about new concepts (Harvey et al., 2016). The students then participated in the process of exploring and building understanding of new concepts using these multimodal materials, examples, or contexts. This helped not only arouse students' interest in the topic (Tomlinson \& Moon, 2013) but also supported students to develop autonomy. 
Even though the teachers worked to change their teaching practices, they knew that the level of student-centredness was limited. Teachers and students reported that they were more familiar with traditional teaching methods. Influenced by Confucianism, Vietnamese HE stresses the importance of teachers as the primary source and transmitter of knowledge in the learning process while students play a passive role as the recipients (Tran et al., 2018). Teachers, therefore, have the ultimate authority and take responsibility for giving knowledge to students who are perceived as unquestioning rote learners receiving knowledge (Tran et al., 2014). This adherence to Confucian principles has resulted in the dominance of a teacher-centred approach in the educational system (Pham, 2010). Teacher-centredness was evident in the EMI classes in this study as despite attempts to promote student-centred learning, the teachers displayed a high level of authority and took charge of students' learning. They relied on the textbook and used question-and-answer techniques in lectures to transfer content knowledge. My classroom observations showed that the teachers and students played traditional roles in knowledge transfer activities and in the one-way interaction style from teachers to students. This supports previous studies that found that a student-centred approach may be adopted on the surface, but teachers and students generally maintain traditional roles in the classroom (Onurkan Aliusta \& Özer, 2017).

In Vietnam, EMI teachers have struggled to shift their pedagogical practices to a studentcentred approach in the face of strong cultural traditions that have established deeply held beliefs. It stands to reason then that when confronted with a new teaching context and insufficient pedagogical support, the subject teachers fell back on their early career and educational experiences. In other words, the teachers appeared to teach in the way they were traditionally taught (Mangan, 2011). This in turn affects the teachers' understanding and implementing of their roles, which eventually leads to few changes in their teaching practices (Tam, 2015). The teachers in my study did not benefit from professional development in innovative EMI teaching methods (Harman \& Nguyen, 2010). Specifically, they were unfamiliar with research-based teaching and learning activities (Tran et al., 2018), and lacked exposure to "practical elements that are needed to enable teachers to adopt and perform student-centred teacher roles in their everyday practices" (Onurkan Aliusta \& Özer, 2017, p. 430). As a result, they selected strategies to fit the new educational context according to their own teaching beliefs and experience rather than adapting their practices in response to professional support in pedagogy (Tran et al., 2018). This implies that teachers in Vietnamese EMI contexts are in need of opportunity for professional development provoking critical reflection, collaboration, 
observation, and action research that may potentially enable them to adopt new knowledge, skills, and beliefs about teaching and learning (Onurkan Aliusta \& Özer, 2017; Tam, 2015).

The students demonstrated a high level of autonomy and independence in their learning, but it was not apparent that they knew how to function in more student-centred classes. Students behaved quite differently inside and outside the classroom. Outside class the EMI students responded proactively to challenges that arose from the new learning context. They searched for effective ways to learn through collaborating with other people in their learning networks. In contrast, when they were in class, the students appeared reluctant to engage in assigned learning activities and seemed passive in the lectures, relying heavily on teachers as the sole source of knowledge. These two contradictory behaviours may represent a conflict between the pervading influence of Confucian philosophy and students' desire to compensate for their limited English proficiency. In Confucianism, students have been taught to respect their teachers by keeping quiet in class and not interrupting the teachers even if they do not understand the topic at all (Pham, 2010). Additionally, the fear of losing face is another cultural factor reportedly preventing students from interacting with both teachers and friends in class, especially when English proficiency is still developing (Pham, 2011). Outside class, access to multiple resources provided digitally able students with opportunities to self-regulate their learning and gain confidence, which appeared to enhance their autonomy and independence.

These factors show a gap between teachers' teaching and students' learning in EMI classes which may hinder students from proactively engaging in classroom activities and fully developing their capacity in learning. The teachers focused on transferring knowledge but did not know how to enable students to take a central role in class. My findings suggest that they could have built on their students' existing skills by integrating digital technologies in their classes and encouraging students' digital competence in learning.

\subsubsection{Content or quality interaction}

Quality interaction increases students' engagement and autonomy, but also enhances students' language skills (Walqui \& Van Lier, 2010). Teaching activities in which students are required to cooperate with each other are considered conducive to learning through another language. Activities like small group discussions, collaborative presentations, and problem-solving tasks are promoted by research as appropriate ways to enhance students' communicative skills in English (Hammond \& Gibbons, 2005). However, to create quality interaction through a foreign language requires teachers to be aware of the language demands of a task, to set specific guidelines for language use, to ensure that group members have clearly defined responsibilities, 
and to provide sufficient scaffolding for students, especially those who possess limited English proficiency or lack confidence. Setting a group task or asking questions to the whole class will not necessarily create opportunities for interaction. The teachers' unfamiliarity with both language features and language teaching pedagogy may explain why the interaction activities conducted in my study were still very limited and why students were not supported to communicate in English. The teachers equated language learning in EMI with business vocabulary development and they were not aware of either the language demands of academic subjects or suitable strategies to teach vocabulary. This is consistent with what other researchers have reported about vocabulary teaching in EMI or CLIL contexts often remaining implicit (Dalton-Puffer, 2008). This indicates that language experts tend to play a limited role in EMI programmes and suggests that collaboration between subject teachers and English teachers may enhance the implementation of EMI courses.

Interaction outside class can also benefit students' learning. According to Hammond and Gibbons (2005), teacher-student interactions can be an effective contingent scaffolding strategy in which teachers respond to students' questions. This may support students' learning and develop their critical thinking (Van Lier, 2004; Wass et al., 2011). Evidence from my study shows that access to digital technologies offered the teachers and students a range of channels for communication and interaction outside classrooms. Nevertheless, this form of interaction between the teachers and students was not designed to support students to improve their English. These interactions were solely for the purpose sharing information, rather than exchanging ideas or interacting with each other to solve meaningful problems. There was an exception when one teacher reported interacting with his students through a social group on Facebook. This activity took place frequently and involved many exchanges relevant to their teaching and learning. Additionally, student-student interaction also plays an important role in students' learning (Schmid et al., 2014). In my study, the interactions among students outside of class via different means of technology appears to have created collaborative learning environments, which may have impacted positively on students' achievement. This suggests that digital interactions could be used more widely to benefit the students in their learning of content knowledge.

Traditional teaching beliefs impacted on teachers' decisions to design interactive tasks in EMI courses. In Vietnam, the education system favours a content-driven approach and teachers are required to focus on subject matter. This is why lectures are the primary means of dissemination of knowledge to students (Tursunov, 2016). Additionally, teachers are always under pressure to cover the vast volume of content mandated in the syllabus and to follow the 
mandated textbooks. Such pressure is exacerbated by the assessment system which heavily emphasises theoretical content knowledge rather than practical skills. As Chapter 5 has discussed, the participant teachers struggled to prepare students for exams at the same time as covering the overloaded syllabus. In this setting, it was not surprising that teachers were not able to integrate opportunities for interaction. For example, Vincent felt so concerned about the students' final scores that he chose to use Vietnamese to ensure his students could understand the subject matter. Nathan reported that he was not able to allocate time for group work in class if the students were to complete the given topics in due time. All of these pressures became barriers preventing teachers from engaging students in quality interactions in class. There are clear implications for policy makers in planning and implementing EMI programmes. Specifically, my findings peak to the need to further consider the balance between language and content instruction, access to teaching materials, and trustworthy assessments.

\subsubsection{English-only or integrating the first language}

The use of the L1 in EMI/CLIL programmes can provide effective scaffolding for learning (Mahan, 2020). Teachers can use the L1 not only to connect students' prior knowledge in the L1 to new knowledge in the L2 (Mahan et al., 2018) but also to allow students to use resources from both languages to understand new concepts (Barr et al., 2012). Strategies like codeswitching (Carter \& Nunan, 2001) or translanguaging (Coyle et al., 2010) have been found to enhance students' content learning (Coyle et al., 2010), facilitate their learning of the target language (Méndez García \& Pavón Vázquez, 2012), and promote teacher-student interaction (Then \& Ting, 2011). In my study, the teachers used Vietnamese for different purposes, mainly to translate academic terms and explain difficult business concepts. Most importantly, they wanted to ensure that their students understood the subject matter.

The teachers appeared to be sensitive about using of L1 in the lectures. This can be attributed to their beliefs and their pedagogical content knowledge. The teachers believed that students would learn English from exposure to English input in EMI classes and therefore tried to avoid using the L1 in lectures and even required their students to use English only in class. However, the teachers justified using the L1 to make their lecture content comprehensible and accessible to students. They used Vietnamese to respond to students when they struggled due to their limited English proficiency. This use of the L1 illustrates how the teachers prioritised their students' academic content learning as they did not use the L1 for the purpose of scaffolding the students' English development. 
No matter how much L1 the teachers used in class, they did not deliberately use this strategy in their teaching activities. Instead, they regularly seemed to make impromptu decisions to switch to Vietnamese when faced with their students' linguistic difficulties or the need explain abstract concepts in their subjects. This is consistent with recent research findings that teachers' practices of using the L1 in EMI/CLIL classes to help students understand complex ideas and notions were "neither systematic nor based on guidelines" (Doiz \& Lasagabaster, 2017, p. 4). The teachers in my study seemed unaware of research-informed strategies for using the L1 in language teaching and learning, even in EMI/CLIL contexts. This also raises the need for a language policy or guidelines for the implementation of EMI programmes in tertiary education settings (Gierlinger, 2015).

\subsubsection{Internationalisation and glocalisation}

In Vietnam, adopting EMI has been considered not only as a positive reaction to the internationalisation of HE (Tran \& Nguyen, 2018) but also as a wise response to the globalisation and regionalisation (Le, 2012) that drive HE institutions to improve their world ranking and meet the demand of local contexts. Common initiatives include promoting English use across institutions, improving students' English skills to meet requirements of foreign recruiters or global market, and enhancing academics' publishing and research collaboration (Dafouz \& Smit, 2020). To implement EMI, Vietnamese policy makers started by examining the academic programmes of high-ranked universities in different countries, then they imported materials from foreign countries, and developed curricula for Vietnamese contexts. These strategies aimed at improving the quality of teaching and learning, enhancing students' and staff professional mobility, and accelerating the process of internationalisation. However, implementing EMI has required a great deal of work from teacher participants in adapting curricula to meet the needs of local students while maintaining the academic values of local institutions. Without this work from teachers, programmes taught at Vietnamese universities may be at risk from the imposition of Westernised approaches and paradigms, and suffer from curricular homogenisation (Leask, 2015).

EMI programmes in Vietnam have not been shaped to accommodate local circumstances. This is evidenced in my study as the key actors in the EMI programmes (the participant teachers and students) were not fully conversant with the rationale for EMI. The levels of teachers' and students' English proficiency did not appear to guarantee the quality of these EMI classes. Although most of the teachers felt confident using English to teach their subjects, their levels of English did not allow them to prepare or deliver their lectures as effectively as they did in Vietnamese. According to Barnard (2014), "the ability to read widely and write at length in a 
second language does not necessarily transfer to effectively explaining key concepts to students in such a way as to make the lectures comprehensible" (Barnard, 2014, p. 15). The students also realised that their own level of English language was not sufficient for them to understand the lectures thoroughly. This aligns with research suggesting that teachers and students participating in content classes in English require a good command both of English in general and of the discourse of their disciplines in particular (Morita, 2004). As Chapter 5 has explained, a gap was evident between the participants' general proficiency in English and their English for academic disciplinary communication.

Another challenge was the teachers' lack of pedagogical knowledge about teaching through English, which ideally would have been addressed in their preparation for EMI teaching. This resulted in the teachers' struggle to engage students in interactive learning activities, and to jointly facilitate students' learning in both content and English.

Textbook-based education is the norm in Vietnam but needs further consideration in the process of glocalising EMI in Vietnam. Vietnamese educators are used to teaching from a textbook and having access to wrap-around materials such as a teachers' guidebook, test-banks, and suggested case-study tasks. Imported curricula using foreign textbooks do not include adequate materials or resources to support their familiar style of textbook-based education and this caused difficulties for the participants. Teachers and students struggled with the subject content of the prescribed English-language textbook, and were uncertain as to how to deal with that content (Forman, 2014). The subject matter included individualistic, aspirational, and Western discourses foreign to Vietnamese and Confucian culture. This left the teachers to adapt these materials as best they could without systematic support. Their experiences align with those of participants in other studies which suggest that materials do not receive adequate attention (Tomlinson, 2012), despite their significant role in tertiary teaching and learning. In my study, evidence reveals that EMI teachers and students compensated for this lack by deploying a range of digital technologies to access multiple resources on the Internet to curate and develop their own teaching and learning resources. This finding calls for a systematic focus in Vietnam on providing appropriate EMI materials and supplementary resources and also supporting teachers in materials development. More importantly, this study shows the significant role that digital resources can play in assisting the teachers and students to adapt to glocalising EMI programmes to fit the Vietnamese context. 


\subsubsection{Summary}

This section has reflected on the implementation of EMI in this Vietnamese context through the ROADMAPPING framework (see Figure 11). The teachers' and students' practices were discussed through the six intersecting dimensions of Roles of English, Academic Disciplines, (language) Management, Agents, Practices and Processes, Internationalisation and Glocalisation. This section took each dimension in turn, drawing on the teaching and learning experiences of teachers and students in these EMI programmes.

\section{Figure 11}

EMI in Vietnamese contexts through the ROAD-MAPPING framework

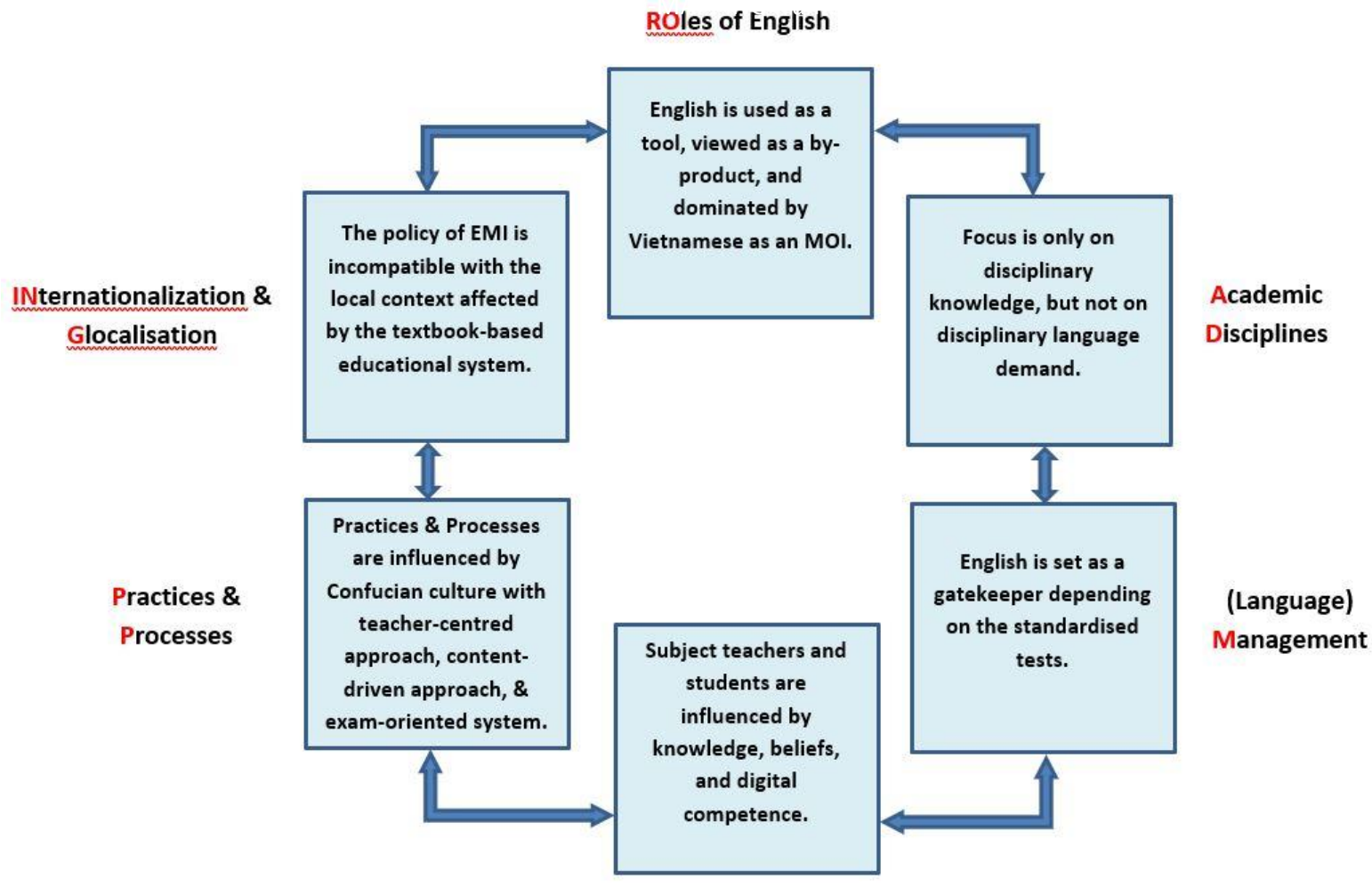

Agents

The participants distinguished several different Roles of English. They thought it was a tool for learning and delivering content, an international language, a language for business study, and a by-product of the content learning process. As a medium of instruction, the role of English was still limited as Vietnamese remained the dominant language in the curriculum. This led to an apparent confusion among the participants about how and how much English should be used in EMI classes.

EMI in Vietnam was applied to more Academic Disciplines, but the priority was on areas which could contribute to the Vietnam's socio-economic development. While each discipline is 
distinguished by specific linguistic conventions and discourses, the teachers as content experts did not seem aware of the pedagogical content knowledge for teaching English and teaching in English. As a result, the students received limited support in language development even though the teachers paid attention to important vocabulary in different content areas.

With respect to Language Management, the participants understood the purpose of promoting English use across the university for academic purposes. English proficiency served as a gatekeeper of students' enrolment or graduation; however, standardised tests such as TOEIC, TOEFL, and IELTS may not have provided the most accurate assessment of the language skills required for studying particular disciplines. The students did not feel that their English levels were appropriate for learning in English in the EMI context and this presented challenges for both the teachers and students.

In the dimension of Agents, the teachers and students were key actors in the EMI setting of my study, bringing their existing knowledge and beliefs to the new learning and teaching experience. The teachers' strong content knowledge and teaching experience led them to prioritise teaching subject matter. This accordingly influenced their students' approaches to learning. Without sufficient English support in the new learning environment, students demonstrated high levels of self-efficacy as they took responsibility for their own learning. Their high levels of digital competence for accessing multiple resources online seemed to facilitate their autonomy in learning.

In Practices and Processes, the teachers and students worked hard to respond to the new language of instruction. The teachers tried different teaching strategies to implement a studentcentred approach. Although the teachers managed teaching and learning in EMI courses, there were few substantive changes in their teaching practices, possibly because of conflicting influences from Confucian educational practices, the belief in a teacher-centred and contentdriven approach, and the exam-oriented system. These factors appeared to inhibit the teachers from changing their teaching practices and also affected students' EMI learning experiences. The students engaged differently with learning activities inside and outside of class. While they still took a passive role of receiving knowledge in class, they proactively self-regulated their learning outside of the classroom by adopting a range of activities, accessing multiple resources, and employing different modes of learning. The students' integration of digital technology in their learning contrasted with their teachers' digital pedagogical practices.

The participant teachers and students in EMI programmes were not conversant with the university policy on Internationalisation and Glocalisation. While EMI was considered as a strategy 
to enhance the institution's world ranking and profile and improve the quality of teaching and learning, the policy makers had failed to fully prepare their teachers and students to meet the new demands on their English proficiency and the adoption of EMI pedagogy, and did not foresee that new teaching and learning materials would be necessary. This created challenges for students and teachers who were used to a textbook-based educational system in EMI contexts.

Despite the increasing popularity of EMI in Vietnam, it is still an emerging phenomenon requiring further investigation. Study findings identify challenges that key stakeholders were facing in EMI programmes. These challenges were derived from the inconsistent top-down policy and different long-established elements in the educational system. Moreover, the implementation of EMI has been influenced by the digital age, neoliberal policies, and the acceleration of internationalisation and globalisation priorities. These influences shape the reality of EMI teaching and learning practices, which will be discussed in detail in the next section.

\subsection{Teaching and learning of EMI courses in the digital age}

In the digital age, the development of technology is changing aspects of society including education (Bates, 2015, p. 14). In HE, universities are required to keep pace with two major trends in the globalised academic world: the on-going technological evolution in teaching and learning; and the diffusion and popularity of EMI (Querol-Julián \& Camiciottoli, 2019). The increasing use of instructional technology and communication tools in HE settings has offered teachers and students not only a range of web-based resources to support their teaching and learning, but also different digital platforms for hosting virtual classes or online courses. The trend of integrating technology into universities runs parallel with the rise of EMI in the process of internationalisation in HE when English is increasingly used as the medium of instruction (Querol-Julián \& Camiciottoli, 2019). The question is how these two significant trends interact with each other in the same educational context. In this section, I attempt to answer this question by discussing how teachers and students' practices in EMI programmes intertwined to adapt to the changing context in Vietnam, especially at a time when an array of technological innovations has been integrated into the educational system. I will first address the impacts of neoliberalism, internationalisation, and globalisation. I will then discuss the pedagogical shift in teachers' knowledge and beliefs, as well as multimodal modes of learning adopted by the students' in their access to technology. Finally, I will examine the agency of both the teachers and students as relates to the implementation of $E M I$ in the digital age. 


\subsubsection{The changing context}

Social changes together with the advances in technology in the digital age have exerted considerable influence on how communities, nations, and societies function and interact (Starkey, 2012). As an important part of any society, the educational system faces the constant challenge of change (Bates, 2015). In this respect, The Strategy for Education Development for Vietnam 2011-2020 mandated an urgent need for reforming the nation's HE system. As a result, it has become a priority for $\mathrm{HE}$ to embrace new learning styles, enhance interactive teaching modes and adopt information and communication technologies into teaching and learning (Harman \& Nguyen, 2010). To do this, most Vietnamese universities are required to implement strategic plans to reform teaching and learning. This has led to a dynamic context in which teachers and students need to respond to different factors such as neoliberalism, internationalisation, and globalisation (see Figure 12).

\section{Figure 12}

The changing context affecting pedagogical practices in the digital age

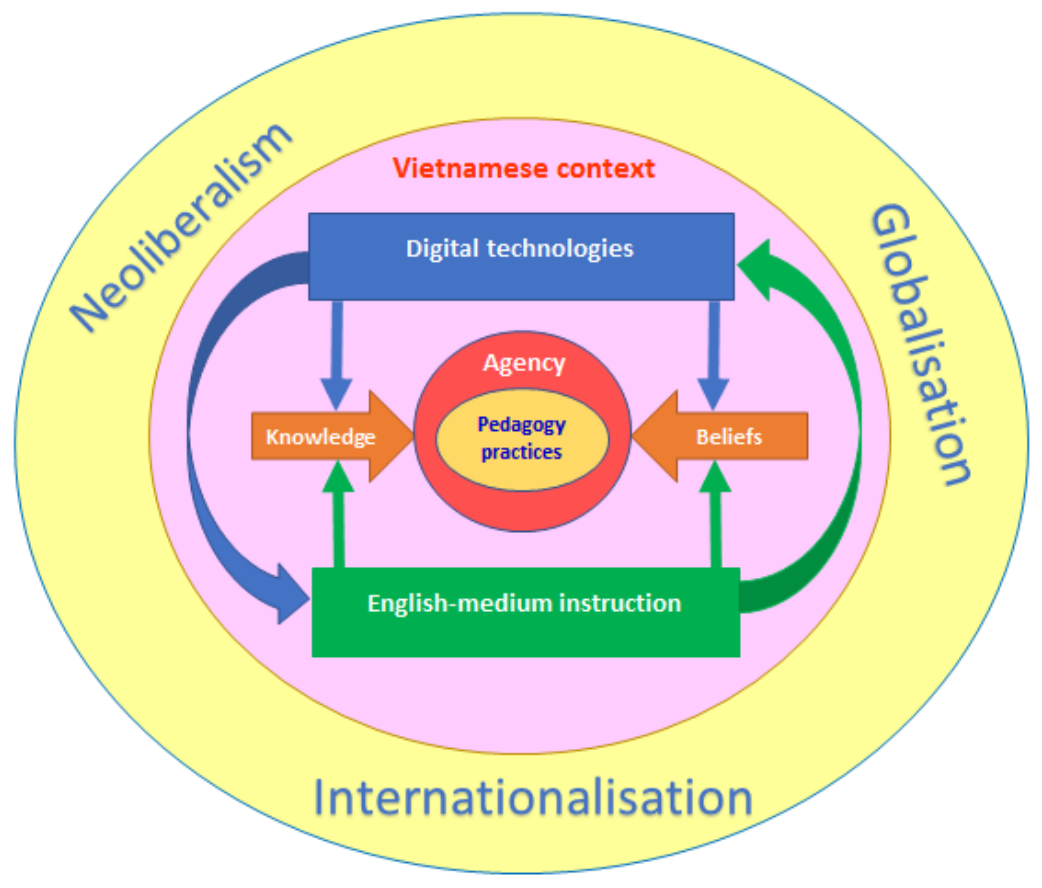

\subsubsection{Neoliberalism, Internationalisation and Globalisation}

In line with globalisation, neoliberalism affects HE. Neoliberalism is a political and economic ideology that "privileges the market as the most efficient platform for distributing social goods and minimizes the role of government responsibility in ensuring collective well-being" (Weis \& Fine, 2012, p. 188). In the neoliberal system, individual responsibility is emphasised while 
support from the state is diminished (Saltman, 2014). Education in a neoliberal context is viewed as a market concern (Golden \& Womack, 2016) or a large cognitive process driven by practices which are codified and commodified to appeal to different groups of learners who are considered as consumers receiving knowledge commodities from teachers (Golden, 2018). In this sense, neoliberalism underpins the privileging of competition and commercialisation of education in $\mathrm{HE}$ (Cannella \& Koro-Ljungberg, 2017). Responding to this ideological system, Vietnamese universities have gained institutional autonomy allowing them to make their own decisions in personnel recruitment, finance, administration, and curricula (Nguyen et al., 2016). This has resulted in $\mathrm{HE}$ institutions including policies promoting internationalisation and EMI programmes. The case study university with its autonomy followed the neoliberal agenda of competing through university rankings and promoting a high-quality university profile. To do this, the policy makers used EMI policy to increase the student intake and accelerate the internationalisation process. As a result, the teachers were expected to adapt to this neoliberal environment by guaranteeing the quality of teaching and satisfying their students as consumers.

Internationalisation driven by globalisation has gained strategic significance in the development of HE institutions across the world (Wihlborg \& Robson, 2018). Universities aim to enhance the quality of education and research, and thus make a meaningful contribution to society (de Wit \& Hunter, 2015). To do this, they tend to utilise technology to accelerate internationalisation and globalisation (Robson, 2016). In Vietnam, internationalisation is viewed as a salient approach to reform the HE system, enhance its capacity and standards, and assist "universities to keep pace with international and regional developments" (Tran \& Nguyen, 2018, p. 94). To do this, $\mathrm{HE}$ institutions have been promoting a range of strategies and policies including the use of English for academic and communication purposes together with integrating digital technology into both administrative and academic activities. At the university research context, alongside the implementation of EMI programmes, a learning management system (LMS) had been introduced as an e-learning platform intended to allow for different forms of teaching and learning. This policy required the teachers to employ technology in their teaching practices.

EMI programmes in Vietnam are intended to prepare students to join a competitive global job market, enhance international collaboration in academia, and improve university profiles and ranking (Lasagabaster, 2017). To achieve these objectives, teachers and students are required to update their knowledge and skills of English. At the university in my research, this is what happened in that the implementation of EMI programmes required teachers and students to adapt to the new context. Yet all the participating teachers and students reported that they 
received very little institutional support to adjust to the EMI environment. This finding reflects a common issue reported in research on EMI in different contexts. For example, Karabassova (2018) explored teachers' conceptualisation of integration of CLIL programmes in Kazakhstan and reported a lack of rationale, communication and preparation from the educational institutions which left the teachers with minimal awareness of the pedagogical intentions behind the programmes, leading in turn to a prioritisation of content knowledge and refusal to each English in the course. This appears to be consistent with Hallinger's (2010) observations about education reform in Southeast Asian countries. Staff are not prepared for the process of educational reform in Southeast Asia and lack skills, readiness and resources to manage change (Hallinger, 2010).

\subsubsection{Pedagogical change}

Adopting EMI in the digital age suggests that teachers are incentivised to adjust their knowledge and beliefs in response to the influences of neoliberalism, internationalisation and globalisation in the digital age. In this section I address teachers' pedagogical shifts through the lens of the TPACK model and highlight significant changes in their digital knowledge. Mishra and Koehler (2006) introduced the TPACK framework by adding technology as a new domain to Shulman (1986) original framework (see Chapter 2). The TPACK framework highlights the intersection of content knowledge, pedagogical knowledge, and technological knowledge and offers sound guidelines for effective teaching with technology (Bibi \& Khan, 2017).

\subsubsection{TPACK}

The TPACK framework integrates technology into teachers' knowledge domains. Teachers need to accommodate all knowledge components to cope with the changing context of the digital age. They need to know how to select appropriate technologies to integrate into teaching specific subject matter to enhancing students' learning. As a context-specific framework, all the TPACK components can be affected by different factors in a particular educational setting.

The necessity of implementing EMI programmes in the digital age has put pressure on teachers' content knowledge. Subject teachers are expected to possess a strong background in content knowledge, but this is changing rapidly due to the development of digital technologies. Thanks to the widespread interaction and access to information through the Internet, knowledge is continually expanded "as new and novel connections open new interpretations and understandings to create new knowledge" (Starkey, 2012, p. 26). This means that each individual is an owner of knowledge in networks where they connect with others to exchange and build up new knowledge. A network in the digital age might include not only an individual's personal network but also virtual networks via the Internet. Additionally, knowledge in the digital network 
is represented and applied in different media such as video, audio, animations, and graphics, and the Internet (Bates, 2015). This consequently leads to a constant change of content knowledge with which teachers must update themselves (Niess \& Gillow-Wiles, 2019). For example, Nathan reported that different groups of experts interpreted topics in his subject differently on the Internet, so he needed to read carefully to direct students to appropriate interpretations. Given that students are exposed to different sources of information online, teachers need to know how to guide them to discern content that is relevant for their learning. This requires teachers to draw on substantive disciplinary knowledge in making pedagogical decisions.

In addition to technology, language is a powerful mediator of academic knowledge (Bates, 2015). Every academic discipline covers a specific body of conventions and assumptions about the knowledge within that subject domain. For this reason, academic knowledge is understood and disseminated through symbolic representation such as language or mathematic symbols. In other words, the language used to represent disciplinary knowledge influences how people understand academic knowledge (Bates, 2015). In EMI programmes, the change of language of instruction from Vietnamese to English has influenced how the subject is understood and challenged the teachers and students in their teaching and learning practices. The teachers needed to learn what and how academic English is used to represent familiar subject matter. Therefore, when the economics subjects were taught in English, the teacher participants required a different level of academic knowledge. This suggests that teachers were expected to learn significantly different content knowledge to teach EMI courses.

Teachers need to know how to teach disciplinary knowledge to students: pedagogical content knowledge. In EMI programmes, teachers need pedagogical content knowledge for teaching that knowledge in English. The Higher Education Reform Agenda issued by the Ministry of Education and Training in Vietnam (MOET, 2014) mandated a change in pedagogy that embraced a student-centred approach and use of digital technologies in tertiary academic programmes including EMI courses. Vietnamese teachers therefore have been under pressure to update their pedagogy by attending workshops, seminars, and conferences. This also entails a change in teacher technological knowledge in order to meet the requirements not only of the policy but also in content knowledge. For instance, teachers in EMI courses need to learn pedagogical strategies appropriate for engaging students in active learning via different modes such as face-to-face, the LMS, giving instruction through English, and explaining business concepts to students. This is a challenge reported by all the teachers in my study. Lack of English proficiency, limited support in accessing essential teaching materials and little pedagogical knowledge in 
teaching in English made them struggle and they tried to address these issues by themselves. Consequently, they deliberately changed elements of their teaching practice to facilitate students' understanding of content knowledge in English, which implies a readiness to adapt their pedagogical content knowledge.

In the digital age, teachers need more than basic technological skills to enhance their pedagogical practices when teaching students with various interests and capabilities (Heitink et al., 2016). Teachers with TPACK are able "to select appropriate technologies that fit with the pedagogy and content in a specific context" (p. 71). My research findings reveal that the teachers actively and deliberately selected and deployed digital technologies in their teaching practices such as scaffolding students' content learning and vocabulary with multimodalities, facilitating their problem-solving and analytical thinking skills with video study cases, or engaging them in collaboration and interaction with quizzes or social network groups. This gradually helped the teachers gain hands-on experience in teaching with technologies and improved their ability to select appropriate technologies to teach specific content knowledge. Most importantly, given their ability to use digital technology to access multiple resources, the teachers made a major pedagogical shift away from textbook-based teaching and took charge of curating and developing their own teaching resources that they believed were relevant for their students.

\subsubsection{Beliefs about teaching with technology}

Teaches' beliefs are a critical factor influencing their decisions about integrating technologies into their teaching practices. The finding from interviews with the teachers and classroom observations in this study shows that the teachers integrated digital technologies into their teaching activities to a limited extent. This could be attributable to their personal beliefs about teaching. Teachers are motivated to integrate technology in their classroom when they see its potential for optimising teaching activities and enhancing students' learning (Joo et al., 2018). When teachers realise this, their beliefs drive teachers to gain TPACK and apply it into their teaching (Maeng et al., 2013). This appeared to be true for Vincent. He wanted to know how to use applications to edit and create subtitles for clips for case study discussions, as he believed that using video cases was effective in class. Conversely, teachers' beliefs might hinder their desire to integrate digital technology in class. For example, Nathan thought that integrating technology in class would be too time-consuming, so he chose not to use it much since the subject matter was already overwhelming. These beliefs about technology, which may have been formed through the teachers" "experiences with technology inside or outside of the classroom and as a student or as a teacher", shaped their pedagogical practices (Farah, 2012). 
Teachers' self-efficacy in using technology affects their pedagogical decisions, as selfefficacy is a significant predictor of teacher use of technology (Li et al., 2019). Teachers tend to use technology when they feel confident in their ability to apply it in classroom activities (Valtonen et al., 2015). In other words, teachers' technology-related self-efficacy impacts their intention to use technology in classroom activities. In my study, while Vincent and Nancy felt digitally competent and frequently used technology in class, Taylor reported that she was not confident in using technology for teaching. It appeared that the teachers' technological knowledge and TPACK affected their self-efficacy in using technology, which is in line with previous studies on the relationship between TPACK and teachers' technology self-efficacy (Joo et al., 2018; Sahin et al., 2013).

Teachers' beliefs about using technology might change in response to contextual factors (Chiu \& Churchill, 2016). If digital technology is available and accessible, teachers are likely to shift their pedagogical practices to include activities not only to engage students in learning but also to stimulate their participation in the process of constructing meaning and knowledge. In other words, accessible technological tools might enable teachers to change their teaching beliefs from a teacher-centred approach towards a more open-ended, student-oriented or constructivist approach (Ertmer et al., 2014). For example, all the teachers in my study endeavoured to include multimodalities and multimedia in their lectures to make them more interactive and engaging to students, although their use was somewhat limited. They made an effort to create opportunities for students to develop higher order thinking skills and gain autonomy in their learning with the assistance of digital technologies. Additionally, classroom observations suggested that the case study teachers also tried to transform their teaching using technologies such as video case discussions, interactive assessments, or social network forums rather than spending class time just lecturing and transmitting knowledge to students. In this situation, the change of instructional language in EMI programmes might have been another influence on teachers' beliefs about the use of digital technology. Particularly, perceiving the significance of business vocabulary in EMI classes, Taylor (albeit without confidence in digital technology) reported designing online quizzes for students to revise terms and concepts. This suggests that teachers make pedagogical decisions in digital technologies according their beliefs about teaching and learning with technology (Tondeur et al., 2017). In my study, the university policy played an influential part in changing the teachers' practices (and possibly beliefs) in facilitating a shift in pedagogy towards studentcentred orientation. This suggests that the university might play a role in supporting teachers to integrate technology in their classes (Tondeur et al., 2017). 


\subsubsection{Multiple modes of learning}

Technological advances in the digital age have offered teachers and students different modes of teaching and learning, each of which offers different affordances (Baragash \& AlSamarraie, 2018). In this study, the students who encountered difficulties in EMI classes reported seeking support and learning autonomously from different digital platforms including the LMS or English Discoveries Online. This supports the results of previous studies that found students in the digital age are able to enhance their learning by independently accessing different learning modes or digital platforms (Hoic-Bozic et al., 2016; Zainuddin \& Perera, 2017) and suggests that teachers would do well to combine different modes of learning to support students (Alducin-Ochoa \& Vázquez-Martínez, 2016). In other words, teachers should consider designing multiple modes of instruction to enhance students' autonomy in their learning.

Digital platforms enable a multimodal learning environment that supports students in EMI courses. Multimodal learning refers to the learning through multiple modes of teaching and learning materials and multiple methods to access information (Bloomfield et al., 2013). In the digital age, these multiple modes and methods include visual, audio, text or speech, and movement channels (Marchetti \& Cullen, 2016). Such modalities are expected to stimulate communication, which potentially enhances learning "by improving interaction between teachers and learners, learners and input materials, and classroom communication in general" (p. 40). In their study, Marchetti and Cullen (2016) found that the students preferred learning new language from image prompts, videos, and visual stimuli which made it easy for them to participate in communicative activities. In my research, the students in EMI courses expressed a preference for frequent use of multimodalities to support learning content knowledge in English. This finding supports studies on EMI / CLIL contexts that multimodal learning can be used as an effective scaffolding strategy (Fernández-Fontecha et al., 2020; Forey \& Polias, 2017).

\subsubsection{Agentic actors}

Teachers and students play the role of agentic actors in response to changes in the new context of EMI. While the teachers displayed their adaptation as agents of changes, the students showed their influences on the teaching and learning practices.

\subsubsection{Teachers as agents of change}

As agents of change, teachers have to be agentic to adapt to the changing institutional context. In a neoliberal institution, educational reforms target economic well-being and competitiveness in a global economy (Bartell et al., 2019). These reforms prioritise branding the institution as a high quality institution with updated pedagogy and professional staff to attract 
both domestic and international student consumers, while adhering to financial policies of raising revenue and reducing costs (Ross \& Gibson, 2007). In the university research context, this created pressure on teachers who are the agents responsible for delivering education and rated on how well they teach (Taylor, 2013). This university had the autonomy to make significant changes to the academic curriculum, management, and students and staff policy. Given their awareness of the university policy of reforming the academic quality through the introduction of EMI programmes, the teachers became agentive in adapting their teaching practices to this policy. For example, when the LMS was mandated for teaching and learning, the teacher participants responded by uploading parts of their lessons onto the e-learning platform even though this added to their workload. This implies that teachers make decisions about how they demonstrate different forms of agency to manage work-related demands (Vähäsantanen, 2013).

The study shows that the teachers worked by themselves to deal with the challenges arising from new policies. This indicates the demonstration of individual agency which reflects the process in which people deliberately bear their influences on their own functioning and the environmental events (MacBlain, 2018). The teachers independently addressed issues in the EMI context rather than seeking support from their managers or collaborating with others. This is an unexpected finding, as Vietnam is known as a collective society in which people are interdependent and relationships are premised on assumptions of common bonds. In contrast, individualism foregrounds personal independence (Mascolo \& Li, 2004). In my study, the teachers showed high levels of autonomy and independence in their job, which enabled them to deal with challenges by themselves. Technological advances provided them with more resources and choices, which allowed them to solve problems independently. Interestingly, this may reflect an apparent transition from collectivism to individualism in Vietnamese society under the growing influence of globalisation and neoliberal ideologies. Economic growth and the availability of multiple resources offers people more choices to make decisions and this seems to encourage individualism, even in a collectivist society (Hofstede, 2001). Another explanation for the teachers' individual efforts may be that the teachers perceived EMI policy to be an institutional duty, which they as a staff member needed to enact as a tribute to the university. Further, in attending collective events such as EMI training courses or syllabus design groups, the teachers demonstrated their collective agency, working with others to achieve things and shape their future (MacBlain, 2018). This may have prepared them for managing EMI courses to a certain extent. Collective agency provides individual teachers with an academic environment so that they can enact their individual agency to take appropriate actions (Fu \& Clarke, 2019). This suggests 
that more opportunities for collaboration are needed to support teachers to work with other colleagues or experts to enhance the quality of teaching, especially in EMI programmes where subject teachers are in need of support to act as an active agent of change.

Despite the teachers' efforts to cope with contextual changes, their professional agency appeared to be limited. Teachers' professional agency is the teachers' ability to act in innovative ways, and even to resist external norms and regulations that are understood to contrast or conflict with professionally justifiable action (Dovemark, 2010). In educational settings, Vähäsantanen (2015) refers to teachers' professional agency from three perspectives : the teacher's opportunity to influence their own work, the teacher's involvement with educational changes, and the teacher's negotiation of professional identity. To be an active agent of change, a teacher needs to have the power to take action and influence their own work, make decisions and choices about their work, and contemplate their professional identities (Vähäsantanen, 2015). In my study, the teachers had very little scope to demonstrate their professional agency in adapting to EMI. They had few opportunities to make decisions associated with the policy of implementing EMI programmes, which was a top-down reform determined by the university. However, the teachers were agentive in deciding how to teach their subjects, organise their classes, and instruct their students. The teachers showed progressive professional agency in their approval of the changes, which in turn led them to actively and innovatively engage with the reform. They all felt motivated to participate actively in the EMI programmes. However, the extent to which the teachers participated in practice varied as the inexperienced teachers participated more energetically than their experienced colleagues. With respect to the negotiation of professional identity, all the teachers were considered pioneers in the new programmes and so assumed a professional identity in line with this trend. They considered participation in EMI programmes as an opportunity to confirm their identity as professionals. Generally, the findings show that the teachers as individuals took a proactive role in EMI programmes, but experienced weak professional agency in changing the process at an institutional level. This suggests that HE institutions could play a greater role in facilitating academics to perform as professionals especially in the new neoliberal environment in Vietnam.

\subsubsection{Students as influential agents}

In a neoliberal university, students, who are considered consumers of educational services, exert significant influence on how academic programmes function. This is reflected through the use of student feedback to evaluate the quality of teaching, learning conditions, course content, and curriculum in HE institutions. The implementation of EMI in Vietnam aimed 
to attract both international and domestic students and prioritised their student customers. Students in EMI programmes therefore act as actors with some influence (Dang \& Moskovsky, 2019) and exercise their agency either individually or collectively (Jiang \& Zhang, 2019). In my study, the students influenced the teachers' decisions in EMI classes. For example, Vincent's students gave him feedback at the end of the first lectures which he used to change his teaching methods. In the case of Nancy, the students negotiated language use in class with her at the beginning of the course. The students' agency might be construed as occurring when they recognise their influence on the teaching and learning activities and their roles as active agents in EMI courses. This is in line with one of Van Lier's (2008) core features of agency: that students are aware of their responsibility within the environment.

Students' agency is enacted through their making choices to do with strategies and resources to handle course-related difficulties $(G a o, 2010)$. All the focus group students displayed their agency in using a range of digital resources and tools to enhance their learning through English. They had a strong sense of agency as proactive learners in the digital age. This finding is partly supported in previous studies where students "enacted agency to use resources and strategies to learn English in the EMI context where English learning is largely left in their own charge" (Jiang \& Zhang, 2019, p. 333). My research findings show a difference in that the student participants' selection of strategies mainly focused on content learning rather than enhancing English competence. This aligns with their subject teachers' emphasis on learning content.

The students' agency in this study is further evident in their self-regulation of their learning. The students in the study developed their own strategies to learn subject matter and overcome difficulties such as challenging content knowledge, and the use of English as the medium of instruction. To do this, they accessed multiple resources in their own time. This finding is in line with the notion that neoliberalism in HE gives "students more choice and more control over learning" (Klemenčič, 2015, p. 20). Students are empowered to be autonomous and gain more responsibility over their learning when technology, especially the Internet, provides them with opportunity to be agentive (Klemenčič, 2015).

Student agency and its development in EMI contexts is enacted in different modes. Even though the students in my study commonly demonstrate individual agency in learning, they also tend to exercise collective agency. The students in my study created a collective personal learning network where they collaborated and interacted with others, which was enabled by social media and digital tools. In this case, collaboration in the networks helped them become agentive in adapting to the new context of EMI. In other words, collective agency supported them to exercise 
individual agency. Moreover, the students with high levels of English demonstrated a strong sense of collective agency by taking responsibility to initiate group work. They proactively organised tasks and allocated members to complete them. On the other hand, the students with limited English proficiency enacted proxy agency - the act of influencing other people with resources and knowledge to take action on their behalf (MacBlain, 2018) - when they encouraged the group leaders to solve a particular problem or secure a particular outcome (Klemenčič, 2015).

\subsection{Summary and implications}

The findings of this study provide insights into the EMI experiences of a group of teachers and students in a Vietnamese university. All the participants believed that they were able to adapt to the new teaching and learning environment where English was used as a medium of instruction in economics courses. The teachers perceived that they could transfer their content knowledge and teaching experience in Vietnamese into the teaching of subject matter through English. However, they were not aware of the specific disciplinary language demands and English teaching pedagogy that accompanied this transfer. They skilfully adapted teaching materials and adjusted teaching strategies to support their students' content knowledge but failed to provide students with support for their English development. This may have resulted from the limited preparation available to teachers participating in the EMI programmes. Even though teachers had high levels of self-efficacy, they lacked pedagogical content knowledge for teaching English and teaching through English, and an understanding of EMI pedagogy. This is likely to have repercussions for the learning opportunities offered in these EMI courses.

The students made the decision to manage their own learning. They started EMI courses with an expectation of improving both content knowledge and English competence. Nonetheless, limited English proficiency, insufficient teacher support and lack of objectives for learning English saw them struggle to learn subject matter through English. They searched for ways to meet the requirements of the course and turned to digital solutions. This did not fulfil their aim of developing English competence but meant that the students took responsibility for their own learning. They accessed multiple resources, prepared for lectures, and created personalised and collective learning networks for support. The students showed autonomy in building their own network in which they could collectively interact and collaborate with other people.

The findings indicate that both the teachers and students were responding to the contextual changes in the digital age. Firstly, the university's neoliberal focus on competition and commercialisation created pressure on the teachers to meet the demand of their consumer students. Secondly, the processes of internationalisation and globalisation required both the 
teachers and students to equip themselves with new skills and knowledge including English competence. Finally, technological advances provided participants access to multiple resources and multimodal platforms that facilitated their adaptation to the changing context. The teachers and students in this study enacted agency in different ways, adopting different roles in the teaching and learning process. Without changing completely, the teachers began to promote a student-centred approach in which they encouraged students to co-construct knowledge, moving away from their traditional role of transmitting knowledge. Meanwhile, the students demonstrated autonomy by proactively using a range of digital technologies to engage in multimodal learning. Although these changes were still in the beginning stages, they can be perceived as demonstrating support for the institutional changes.

Generally, the study's key findings show the teachers' and students' uses of digital technologies for various purposes in coping with challenges in the emerging context of EMI. Both the teachers and students turned to be agentic in response to contextual changes influenced by globalisation, internationalisation, neoliberalism, and the digital age. The integration of English as a medium of instruction made them change their teaching and learning practices; and the deployment of digital technologies enabled them to make those changes. This provides significant insights into practices in EMI classrooms in the Vietnamese context.

Study findings derived from the multifaceted combination of teachers' and students' perspectives and voices are valuable in highlighting the nature of EMI implementation in the Vietnamese context. The introduction of EMI programmes at the university is aligned with the orientation of the Ministry of Training and Education in which the promotion of English use is prioritised in domains meeting the socio-economic development requirements such as economics. However, the reality of implementing EMI appears to be a shortcut accelerating the process of internationalisation, which reveals gaps between the policies and the real practices. The insufficiency of preparation and support for teachers and students, the lack of teaching and learning resources, the inconsistency in the EMI curriculum and language policy are some of the challenges hindering the success of EMI implementation. This highlights the extent to which national and institutional policies connect with the affordances and constraints of the local Vietnamese context and calls for the reconsideration of the EMI policies at all levels in which there should be a combination of top-down and bottom-up directions to involve voices of all significant stakeholders in English-medium education.

The ROAD-MAPPING framework has provided a valuable lens to scrutinise the findings of the study. Dafouz and Smit (2020) described ROAD-MAPPING as a conceptual framework not only 
for addressing language-related concerns in EMI contexts but also for exploring the changes and actions that may take place in these contexts. Using this lens, the study's findings have revealed crucial implications for theoretical research, institutional policies, and EMI practices, each of which is discussed further below.

\subsubsection{Theoretical implications}

The theoretical framework of ROAD-MAPPING has highlighted the multifaceted nature of EMI programmes in this Vietnamese context. Reflecting the teachers' and students' practices through the six intersecting dimensions of ROAD-MAPPING, the study revealed the tensions and synergies between the EMI policy promoted as a strategy for internationalisation and globalisation in the local neoliberal setting. This study highlights the need for research on how local forces affect the glocalisation of EMI programmes. These influences include:

- academic roles of English;

- educational reforms;

- impacts of cultural factors on education;

- $\quad$ social demands of the local institutional and workplace contexts;

- and knowledge and beliefs of key agents (teachers and students).

The study makes a further contribution to the field in the explicit recognition and exploration of the role of digital technologies in the developing Vietnamese EMI setting. This focus sheds important light on ways in which participants harness the affordances of digital technologies as they address the constraints arising from the process of implementing EMI. The initiatives shown especially by student participants speak directly to globalised HE where digital technologies play a significant part in shaping and accelerating societal change.

\subsubsection{Institutional policies}

The study also has significant implications for EMI policy makers in Vietnam. One implication is that a coherent language policy is necessary for developing and adopting EMI courses in academic programmes. The language challenges and constraints reported by the teachers and students in this study demonstrate that EMI requires teachers to engage in a process that is more complex than translating lectures into another language. Policy makers need to support content teachers to learn more about the how languages work and how content is taught in another language: pedagogical language knowledge if students are to learn language as well as content. Another implication relates to promoting digital technologies to enhance teaching and learning. While the university had invested in digitising the academic environment, the teachers and students' use of educational technologies was variable. This suggests that policy makers 
should re-examine the adoption of EMI with a view to integrating pedagogically valuable technologies. The study's findings point to three particularly valuable areas for this integration, namely, curriculum design, teacher and student support, and teaching and learning resources.

Firstly, the change from teaching in Vietnamese to teaching in English requires further careful consideration in designing the curriculum. As Chapter 5 has shown, the current curriculum revealed an absence of objectives for the development of English. In addition, despite being promoted by the policy, the e-learning system tended to be underused by both the teachers and students. Given these constraints in the implementation of EMI courses, institutional policy makers would do well to consider the following:

- revising the curriculum, supporting content teachers with professional development relating to how language is taught, and attending to the integration of digital technologies;

- revising the language policy through discussions with content and language teachers to specify a guideline on language use in EMI classrooms;

- encouraging a collaborative approach between content and English specialists to plan how English should be used for different purposes in the course (e.g., content delivery, classroom interaction, and assessment);

- developing a specific model of integrating the virtual learning environment into the curriculum so that teachers and students learn how to utilise various digital platforms and modes of learning in the process of teaching and learning.

Secondly, policy makers need to support teachers and students in EMI courses. Teachers and students are required to demonstrate a certain level of English proficiency to perform their teaching and learning through English. In this study, teachers and students agreed that their English proficiency was inadequate to allow them to take full advantage of EMI. The institutional policy needs to consider supporting students with their English learning so that they can fully participate in EMI courses. This might be achieved in the following ways:

- implementation of a language support scheme, or a tutoring programme operating alongside EMI classes;

- provision of English modules in which students are able to access extra help from English mentors;

- availability of reference materials such as English worksheets or subject glossaries;

- EMI policy revision of English modules, so they align better with subject matter; 
- accreditation of English specialists to play a role in the implementation of EMI;

- greater connection between English modules and EMI courses and collaboration between subject teachers and English teachers to enhance the language support for students, lessen pressure on EMI teachers, and increase the effectiveness of EMI in enhancing both content and language learning.

The teachers wanted more practical support. These Vietnamese EMI teachers were content specialists and non-native speakers of English who felt that their command of English would be sufficient for teaching their subject through English. As EMI was new to these teachers, however, they lacked knowledge and skills related to EMI pedagogy, and pedagogy for teaching English and teaching through English.

Another issue related to the teaching of EMI in the digital age is that many teachers were not prepared to integrate digital technology into their teaching to meet the needs of a generation of learners with good digital competence. These teachers in the EMI programmes required opportunities for professional development to develop their English, update their pedagogical knowledge for EMI, learn how to use digital technology effectively for teaching in the digital age, and develop agency to enhance their performance in the changing context. The university might take the following on board:

- offering a range of professional development activities such as workshops or seminars in EMI practices and pedagogy, TESOL, and digital technology in teaching and learning;

- promoting community of practice for teachers to share with each other;

- or providing subject teachers with one-on-one consultations with EMI experts.

Teaching and learning resources play an important role in the adoption of EMI in Vietnamese contexts. The use of imported textbooks is prevalent, and these textbooks do not include topics relevant to the local setting. This suggests that the policy of selecting imported textbooks for the curriculum and imposing them on teachers and students may create challenges for EMI. The university should consider the following:

- empowering teachers to adapt and develop teaching resources to fit the local environment;

- supporting the development of digital resources to inform EMI programmes. and seeking teachers' active engagement in the process of implementing appropriate changes in designing and developing materials for EMI courses. 


\subsubsection{EMI practices}

Teachers and students in EMI programmes have to change their teaching and learning practices to work in the digital age. An effective EMI curriculum is designed to promote a studentcentred environment where students develop the autonomy to learn both language and content (Wilkinson, 2011). EMI teachers and students need to orient their practices towards studentcentred learning. They need to adopt different roles in teaching and learning so teachers become facilitators that understand how to promote language learning as well as subject learning and students are proactive actors co-constructing new knowledge. Improved access to multiple resources can facilitate their control of multimodal teaching and learning materials to develop both English and content knowledge. Additionally, the availability and accessibility of different learning modes offering teachers and students a multimodal learning environment may stimulate a change in practice appropriate for EMI contexts (as shown in Figure 13). To do this, the teachers and students need to exploit the full potential of technological advances in the digital age to support their teaching and learning activities.

\section{Figure 13}

Digital technologies affecting EMI practices

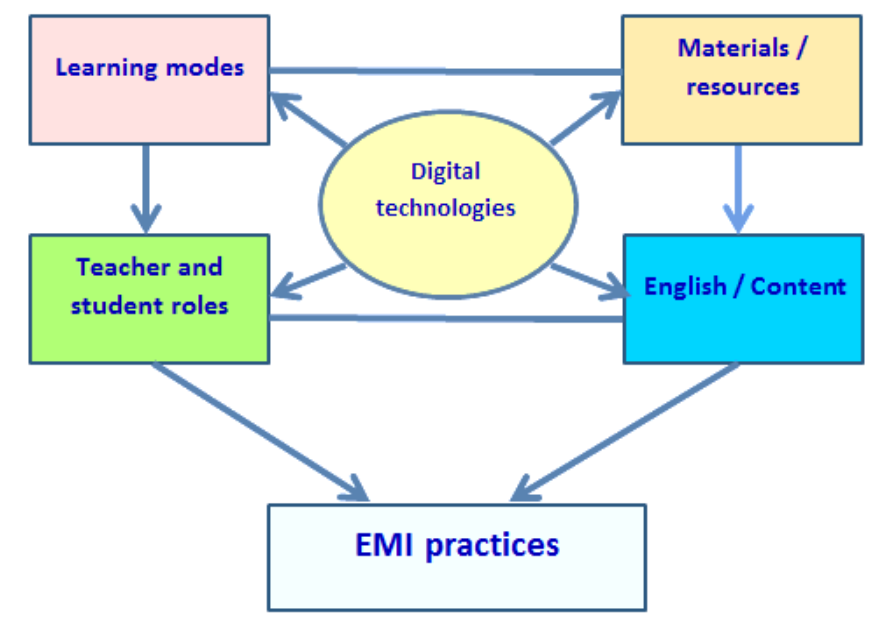

The findings of the study have yielded practical implications for teachers in EMI contexts. Teachers may benefit from an understanding of the potential of constructivist teaching strategies to enhance students' comprehension of content knowledge, facilitate their analytical thinking and problem-solving skills, and engage them in interaction and collaboration. The teachers may find it useful to do the following:

- learn to recognise language demands and linguistic features of their disciplines; 
- learn English teaching strategies applicable for EMI such as code-switching and translanguaging in which they can discuss the use of L1 with students in class and deliberately use L1 as a language resource;

- maximise classroom activities that provide opportunities for their students to interact with each other in English

- engage in communities of practice and intentionally collaborate with other colleagues for professional development;

- learn how to integrate digital technology in scaffolding students' learning of not only content but also English.

The students in EMI courses demonstrated autonomy and exercised agency in the new learning contexts. They possessed strong digital competence and knew how to utilise it in selfregulating their learning and personalising learning networks. However, lack of preparation and investment in English development challenged their learning in this EMI setting. This suggests that EMI students need to focus on the following:

- continue improving their English proficiency by maximising their use of English in classroom activities where there are opportunities to interact with each other in English;

- deploying their collective learning networks and social interactions outside classrooms to enhance communicative skills in English as well;

- and taking a proactive role in order to accomplish the dual objectives of content and language learning in the EMI context. 


\section{Chapter 7. Conclusion}

English medium instruction (EMI) is a global trend in higher education which coincides with the digital age. This study explores the implementation of EMI in the digital age in a Vietnamese higher education (HE) context. In this chapter, I return to my research questions which were addressed throughout the thesis. I then outline the contributions that the study findings bring to the research area, before detailing some limitations of the study and recommendations for future research.

This thesis examines the uses of digital technologies in an EMI context in Vietnamese HE. Within this focus, the study investigates three sub questions, namely around how teachers and students use digital technologies, and how teachers and students perceive students' learning to have developed through digital technologies in an EMI environment. Findings show that the teachers and students deployed a range of digital technologies for teaching and learning such as digital devices (computers, laptops, smartphones, tablets), search engines (Google, Wikipedia), presentation tools (PowerPoint, Prezi), organisation tools (Google drive, Dropbox), social networks (YouTube, Facebook), and the learning management system (LMS). The purposes for integrating these technologies into their practices varied from accessing multiple resources, delivering information, to interacting and collaborating with other people. These uses of technology functioned to support teaching and learning when EMI was at the early stages of implementation.

Study findings provide evidence that teachers and students used digital technologies for EMI teaching and learning in different ways. The teachers tended to use technology to curate and develop materials, present subject matter, organise classes, and communicate with students. They believed that using technology to supplement EMI practices improved their students' content knowledge, their learning of English vocabulary, as well as their engagement and motivation. As for the students, they used technology to search for materials, upload and download information and resources, and to organise lesson content. They proactively utilised technology to personalise their learning by accessing informal online activities and engaging with collective learning networks, which enabled them to collaborate and gain support for learning. The students believed that digital technologies played an integral part in enhancing their understanding of subject matter and improving their English vocabulary and skills. The study found that the use of digital technologies helped satisfy some criteria of EMI courses with respect to the rigour of academic content, high expectations, and the opportunities to engage with different modes of learning in the courses. Quality interaction was achieved through the students' 
digital networks outside classes. Those findings contribute to the area of EMI implementation in the digital age which may be facing changes in a globalised world. Since relatively little research has investigated the use of EMI at the level of practice in an East Asian context such as Vietnam, or the use of digital technologies to assist with the successful delivery of a CLIL/EMI curriculum, it is hoped that the study has made a valuable contribution to the field.

\subsection{Contributions of the study}

This study extends understanding of international trends in pedagogy in response to a changing global context. Increasingly, globalisation and rapid technological changes in the 21st century call for the rethinking of pedagogy in education (Crawford, 2017). One of the challenges that HE faces is how to foster students' ability to communicate, reason effectively, judge the accuracy of information, solve problems, and collaborate in diverse teams (Pellegrino et al., 2001). According to Starkey (2012), technologies in the digital age have facilitated a shift in the acquisition of knowledge from a positivist position to a constructivist position. This means that learners are more likely to actively engage in meaning and knowledge construction rather than passively receiving information transferred from their teachers. Therefore, it is important that teachers move beyond transmission-based teaching or teacher-centred approaches toward transformative teaching or learner-centred approaches in their practices (Tarling \& Ng'ambi, 2016). In this study, the deployment of digital technologies enabled teachers to make changes in their teaching practices to accommodate the challenges of EMI teaching. Access to digital resources appeared to facilitate change for these teachers. The teachers deliberately used digital technologies in their classes to make new content accessible to learners, facilitate their analytical thinking, engage them in problem solving tasks, and encourage them to collaborate in interactive activities. They tried to create a student-centred learning environment in which students could develop high order thinking skills and autonomy by using technologies in learning. This changing practice demonstrates the reality of EMI development in Vietnamese HE towards a technologyenabled pedagogy. This finding not only informs would-be participants in EMI of EMI practices in actual classroom contexts but also reflects changing teaching practices in response to the changing context of HE.

This research reveals drivers for change to teachers' practices in EMI courses. In education, teachers not only make their pedagogical decisions according to their technical, content, and pedagogical content knowledge and beliefs about teaching their subject, but they also change their practice in response to external social pressures including an imperative to keep up with technological change (Tynjälä \& Gijbels, 2012). For example, changes in the world of work 
requires teachers to change their teaching practices to prepare students with essential skills and knowledge needed in the global job market. The development of digital technologies is a facilitator of teaching and learning changes (Tynjälä \& Gijbels, 2012). In an educational environment where teachers have strong knowledge on technology, pedagogy and content and access to technological resources, there are opportunities for them to clarify their pedagogy and use technology to improve their instruction (Trinidad \& Ngo, 2019). Apart from the institution and global context, a new generation of technologically-savvy learners are likely to push their teachers to keep up with this fast paced change and adapt their teaching to provide motivation and a necessary challenge (Fullan \& Langworthy, 2013).

The teachers in my study endeavoured to adapt their teaching in response to changes in practice demanded by EMI courses and the neoliberal HE system. The change of language of instruction affected not only the nature of their subjects but also the pedagogical practices used to communicate content knowledge to learners. Given a textbook in English without teachers' guidelines or reference resources, the teachers worked to assist students to comprehend subject matter presented in English. They therefore searched for new ways to mitigate the changes brought about by EMI. They took advantage of access to multiple resources offered on the Internet and this access made a valuable contribution to their teaching practice in EMI courses. However, the pedagogical decisions the teachers made were limited or enhanced by their ability to use EMI pedagogies and also their skills in connecting technology to pedagogical practices. They mainly used technology as supplementary tools to support their teaching activities or maintain their usual practices. There were few differences in the pedagogy that the teachers applied in their practice to enable the transformation of teaching. I have argued that this is possibly because of the Confucian influences on educational practices, a belief in a teacher-centred and contentdriven approach, and the exam-oriented system, all long established in Vietnamese education. This suggests important implications in the need for provision of professional development opportunities. In particular, teachers can be supported in enhancing their technological pedagogical knowledge through training in selecting or developing context-specific teaching materials.

Learning in a new context commonly requires students to take more responsibility for their own learning. In the digital age, students may have to participate in unfamiliar and changing classroom environments; interact and collaborate in student-centred tasks, problem solve, direct their own learning, and take different roles in the learning process (Kennewell et al., 2008). To do this, they need support from digital resources or people around them such as parents, teachers, 
and peers. The students in this study faced the additional challenge of learning economics through a foreign language, a novel experience for all participants. Receiving insufficient language support from the teachers and the institution, they managed to cope with the many changes in the learning context by themselves. The findings indicate that the students displayed a high level of digital competence in managing their learning and seeking support outside class. Therefore, teachers in the new learning context should consider how they can support to develop their students' learning autonomy.

Study findings show that the teachers and students became agentic as they adapted their teaching and learning to the new EMI context. In education, teachers play an important role as agents of change. To demonstrate agency when facing change, teachers need to take intentional action to make significant differences (Toom et al., 2015), nurture their professional identities and innovate their professional practices (Day \& Kington, 2008). In this study, the teachers made independent pedagogical decisions and took a proactive role in facing challenges in the EMI environment. Alongside personal factors like beliefs and knowledge, easy access to multiple resources from digital technologies enabled them to address issues in the context. The students had a strong sense of agency as proactive learners in the digital age. They were autonomous and collective in their learning and made innovative use of technology in the EMI environment, which in turn resulted in collective support in coping with the new EMI environment.

The teachers and students applied their autonomy differently in dealing with the changing context. While the teachers managed to face challenges by themselves, the students tried to seek help through social interaction and collaboration. From a cultural perspective, teachers and students from collective societies often try to change themselves to fit into a context (Jiang, 2016). This might explain why the teachers and students in my study attempted to adapt independently to the EMI programmes despite understanding them as the responsibility of the whole university. From another perspective, collectivism also views academic learning as a social process that includes family, peers, community, school settings, and larger social institutions (Jiang, 2016; Mascolo \& Li, 2004). The students as autonomous learners were prone to collective learning network to manage their learning. Meanwhile, the teachers tended to conduct little collaborative work in their teaching possibly because of their high levels of autonomy. This raises some implications not only for institutional policy for professional development which encourages teachers' collaboration but also for the learning support scheme and teaching practices which offer students opportunities to access collaborative support and tasks. 
The main strength of this study is it presents the findings in a broader light and reflects upon the implications of national EMI policy and pedagogy practices. The ROAD-MAPPING framework (Dafouz \& Smit, 2020) was used to explore the characteristics of EMI programmes implemented in this Vietnamese HE context. In particular, the dimensions of Agents, and Practices and Processes highlighted the teachers' pedagogical decisions and students' experiences in the English-medium courses. Evidence revealed that the participants made significant changes to their teaching practices to achieve the content learning objectives of their courses. These changes seemed attributable to their knowledge and beliefs, the availability of teaching and learning resources, the institutional EMI policies, and the local educational systems. These influences related to four further dimensions of ROAD-MAPPING, namely Roles of English, Academic disciplines, Language Management, and Internationalisation and Glocalisation. Through the lens of the framework, this study's findings highlight the multifaceted nature of EMI programmes in the Vietnamese context, which contribute to the comprehensive analysis of EME realities.

This study reveals the significant impact of glocalisation in shaping EMI policies in Vietnamese $\mathrm{HE}$ institutions. The introduction of EMI at the participating university was the policy makers' response to internationalisation where global academic programmes were imported into this local Vietnamese HE institution. A number of contextual factors influenced the process of EMI implementation such as the role of English as language of instruction in most academic programmes, the textbook-based system, the preparation for both subject teachers and students, and the importance of English development and requirement in EMI curriculum and language policy. Despite the increasing popularity of English as a global language, it is still considered a foreign language in Vietnam and Vietnamese continues to be the predominant language of instruction in most academic programmes. Vietnamese teachers and students have always relied heavily on assigned textbooks for the courses. Teaching from an imported textbook written in English created a challenge for teachers and students. Changing to English as a medium of instruction therefore requires the sourcing and development of teaching and learning materials, and elaborate preparations by teaching staff. There is also a need to emphasise the development and provision of a learning support scheme for students in the system.

This study provides empirical support for the view that there tends to be a programme expectation that teachers and students should have high proficiency English levels in EMI. However, actual English language proficiency does not always match the English language demands of the content materials. This is consistent with the policy makers' and teachers' lack of awareness that language practices are specific to particular disciplines. In addition, both the policy 
makers and academics are unaware of the specific pedagogy required for EMI teaching and entirely overlook the language component of EMI in the implementation. This results in subjectdominant EMI teaching practices in that the teachers and students focus on disciplinary knowledge and expected English skills to follow. Addressing this mismatch requires more nuanced policies not only for teacher recruitment and student admission requirements, but also in supporting the language needs of both teachers and students in the process of implementing EMI programmes. All in all, there are special characteristics in the Vietnamese education context that complicate the implementation of these programmes. This suggests that the synergy of 'global' and 'local' factors needs careful attention if EMI is to work in practice.

\subsection{Recommendations for further research}

This study raises some recommendations for further research. First, there is a need for research investigating how EMI works in Vietnamese HE contexts over time. While this study reveals the characteristics of EMI programmes at the beginning stages of implementation, it is crucial to reflect on how they change in the local context. The findings from this study could be supplemented with findings from larger-scale research projects as the participation of more stakeholders and institutions would offer a comprehensive landscape of EMI in Vietnamese HE. Second, further research is in need to measure the effectiveness of different aspects in the EMI environment. While a few studies explore EMI via participants' perceptions and observations, little literature focuses on assessing the students learning of the components of both language and content, or the influences of digital technologies on students' learning during EMI classes. This may require the development of specific qualitative or mixed methods measurement tools and experimental research methodology. Finally, researchers should consider examining EMI pedagogy in the digital age to meet the changing global context. Research foci such as these could inform the implementation of EMI in higher education that meets the demands from internationalisation and globalisation. 


\section{References}

Admiraal, W., Westhoff, G., \& de Bot, K. (2006). Evaluation of bilingual secondary education in the Netherlands: Students' language proficiency in English 1. Educational Research and Evaluation, 12(1), 75-93. https://doi.org/10.1080/13803610500392160

Aguilar, M. (2017). Engineering lecturers' views on CLIL and EMI. International Journal of Bilingual Education Bilingualism, 20(6), 722-735.

https://doi.org/10.1080/13670050.2015.1073664

Aguilar, M., \& Muñoz, C. (2014). The effect of proficiency on CLIL benefits in Engineering students in Spain. International Journal of Applied Linguistics, 24(1), 1-18. https://doi.org/10.1111/ijal.12006

Ahmad, S. Z. (2016). The flipped classroom model to develop Egyptian EFL students' listening comprehension. English Language Teaching, 9(9), 166-178.

\section{https://doi.org/10.5539/elt.v9n9p166}

Airey, J. (2011). Talking about teaching in English: Swedish university lecturers' experiences of changing teaching language. Ibérica: Revista de la Asociación Europea de Lenguas para Fines Específicos(22), 35-54.

Akinde, T. A., \& Adetimirin, A. E. (2019). Administrative and infrastructural factors affecting library and information science educators' actual use of educational support systems for teaching in Nigerian universities. Journal of Librarianship and Information Science, 51(1), 180-195. https://doi.org/10.1177/0961000618773140

Al-Baekani, A., \& Ridwan, I. (2018). Integrated of mobile phone as interactive media in extensive listening. SHS Web of Conferences, 42(9), 1-7.

\section{https://doi.org/10.1051/shsconf/20184200006}

Al-Hariri, M. T., \& Al-Hattami, A. A. (2017). Impact of students' use of technology on their learning achievements in physiology courses at the University of Dammam. Journal of Taibah University Medical Sciences, 12(1), 82-85.

https://doi.org/10.1016/i.jtumed.2016.07.004

Al-Masheikhi, F., Al-Mahrooqi, R., \& Denman, C. J. (2014). Investigating college of science student attitudes towards using English as a medium of instruction. The $2014 \mathrm{WEI}$ International Academic Conference, New Orleans, USA.

Alducin-Ochoa, J. M., \& Vázquez-Martínez, A. I. (2016). Academic performance in blendedlearning and face-to-face university teaching. Asian Social Science, 12(3), 207-221. https://doi.org/10.5539/ass.v12n3p207 
Ali, F., \& Ahmad, W. (2017). Effect of technology enhanced language learning on vocabulary acquisition of EFL Learners. International Journal of Applied Linguistics \& English Literature, 6(3), 262-272. https://doi.org/10.7575/aiac.ijalel.v.6n.3p.262

Ali, N. L. (2013). A changing paradigm in language planning: English-medium instruction policy at the tertiary level in Malaysia. Current Issues in Language Planning, 14(1), 73-92. https://doi.org/10.1080/14664208.2013.775543

Ángel, M.-A., Ana María Duarte, H., María Dolores Guzmán, F., \& Ignacio, A. (2019). Mobile learning in university contexts based on the unified theory of acceptance and use of technology (UTAUT). Journal of New Approaches in Educational Research, 8(1), 7-17. https://doi.org/10.7821/naer.2019.1.317

Angela, N.-E., \& Alejandra, C.-T. (2018). Pedagogical uses of digital technologies by pre and inservice teachers: Literature review from a global perspective to the Chilean context. Proceedings, 2(21), 1335. https://doi.org/10.3390/proceedings2211335

Baguma, R., Bagarukayo, E., Namubiru, P., Brown, C., \& Mayisela, T. (2019). Using Whatsapp in teaching to develop higher order thinking skills: A literature review using the activity theory lens. International Journal of Education and Development using Information and Communication Technology, 15(2), 98-116.

Baker, W., \& Hüttner, J. (2017). English and more: A multisite study of roles and conceptualisations of language in English medium multilingual universities from Europe to Asia. Journal of Multilingual Multicultural Development, 38(6), 501-516.

https://doi.org/10.1080/01434632.2016.1207183

Bandura, A. (1986). Social foundations of thought and action: A social cognitive theory. Englewood Cliffs, N.J. : Prentice-Hall.

Bandura, A. (2010). Self-efficacy. The Corsini encyclopedia of psychology, 1-3.

\section{https://doi.org/10.1002/9780470479216.corpsy0836}

Banegas, D. L. (2020). Content knowledge in English language teacher education: International experiences (1st ed.). Bloomsbury Publishing.

Baragash, R. S., \& Al-Samarraie, H. (2018). An empirical study of the impact of multiple modes of delivery on student learning in a blended course. The Reference Librarian, 59(3), 149162. https://doi.org/10.1080/02763877.2018.1467295

Barnard, R. (2014). English medium instruction in Asian universities: Some concerns and a suggested approach to dual-medium instruction. Indonesian Journal of Applied Linguistics, 4(1), 10-22. 
Barr, S., Eslami, Z. R., \& Joshi, R. M. (2012). Core strategies to support English language learners. The Educational Forum, 76(1), 105-117. https://doi.org/10.1080/00131725.2011.628196

Bartell, T., Cho, C., Drake, C., Petchauer, E., \& Richmond, G. (2019). Teacher agency and resilience in the age of neoliberalism. Journal of teacher education, 70(4), 302-305. https://doi.org/10.1177/0022487119865216

Başıbek, N., Dolmacı, M., Cengiz, B. C., Bür, B., Dilek, Y., \& Kara, B. (2014). Lecturers' perceptions of English medium instruction at engineering departments of higher education: A study on partial English medium instruction at some state universities in Turkey. ProcediaSocial Behavioral Sciences, 116, 1819-1825.

\section{https://doi.org/10.1016/i.sbspro.2014.01.477}

Bataineh, R. F., \& Mayyas, M. B. (2017). The utility of blended learning in EFL reading and grammar: A case for Moodle. Teaching English with Technology, 17(3), 35-49.

Bates, T. (2015). Teaching in a digital age: Guidelines for designing teaching and learning for a digital age. BC Open Textbooks.

Baumert, J., Kunter, M., Blum, W., Brunner, M., Voss, T., Jordan, A., Klusmann, U., Krauss, S., Neubrand, M., \& Tsai, Y.-M. (2010). Teachers' mathematical knowledge, cognitive activation in the classroom, and student progress. American educational research journal, 47(1), 133-180. https://doi.org/10.3102/0002831209345157

Belhiah, H., \& Elhami, M. (2015). English as a medium of instruction in the Gulf: When students and teachers speak. Language Policy, 14(1), 3-23. https://doi.org/10.1007/s10993-014$\underline{9336-9}$

Berrett, B., Murphy, J., \& Sullivan, J. (2012). Administrator insights and reflections: Technology integration in schools. The Qualitative Report, 17(1), 200-221.

Bibi, S., \& Khan, S. H. (2017). TPACK in action: A study of a teacher educator's thoughts when planning to use ICT. Australasian Journal of Educational Technology, 33(4), 70-87. https://doi.org/10.14742/ajet.3071

Bloomfield, J. G., Cornish, J. C., Parry, A. M., Pegram, A., \& Moore, J. S. (2013). Clinical skills education for graduate-entry nursing students: Enhancing learning using a multimodal approach. Nurse Education Today, 33(3), 247-252.

\section{https://doi.org/10.1016/i.nedt.2011.11.009}

Bond, M., Buntins, K., Bedenlier, S., Zawacki-Richter, O., \& Kerres, M. (2020). Mapping research in student engagement and educational technology in higher education: A systematic 
evidence map. International Journal of Educational Technology in Higher Education, 17(1), 1-30. https://doi.org/10.1186/s41239-019-0176-8

Bond, M., Marín, V., Dolch, C., Bedenlier, S., \& Zawacki-Richter, O. (2018). Digital transformation in German higher education: Student and teacher perceptions and usage of digital media. International Journal of Educational Technology in Higher Education, 15(1), 1-20. https://doi.org/10.1186/s41239-018-0130-1

Borg, S. (2016). English medium instruction in Iraqi Kurdistan: Perspectives from lecturers at state universities. British Council.

Bozdoğan, D., \& Karlıdağ, B. (2013). A case of CLIL practice in the Turkish context: Lending an ear to students. Asian EFL Journal, 15(4), 89-110.

Bradford, A., \& Brown, H. (2018a). English-medium instruction in Japanese higher education: Policy, challenges and outcomes. Multilingual Matters. https://doi.org/10.21832/9781783098958

Bradford, A., \& Brown, H. (2018b). ROAD-MAPPING English-medium instruction in Japan. In A. Bradford \& H. Brown (Eds.), English-medium instruction in Japanese higher education: Policy, challenges and outcomes (pp. 3-13). Multilingual Matters. https://doi.org/10.21832/9781783098958

Bray-Clark, N., \& Bates, R. r. (2003). Self-efficacy beliefs and teacher effectiveness: Implications for professional development. Professional Educator, 26(1), 13-22.

Brisk, M. E. (2014). Engaging students in academic literacies: Genre-based pedagogy for K-5 classrooms. Routledge.

Bruner, J. (1985). Child's talk: Learning to use language. Child Language Teaching and Therapy, 1(1), 111-114.

Bruton, A. (2011). Are the differences between CLIL and non-CLIL groups in Andalusia due to CLIL? A reply to Lorenzo, Casal and Moore (2010). Applied Linguistics, 32(2), 236-241. https://doi.org/10.1093/applin/amr007

Cannella, G. S., \& Koro-Ljungberg, M. (2017). Neoliberalism in higher education: Can we understand? Can we resist and survive? Can we become without neoliberalism? Cultural Studies $\leftrightarrow$ Critical Methodologies, 17(3), 155-162.

\section{https://doi.org/10.1177/1532708617706117}

Caprara, G. V., Fida, R., Vecchione, M., Del Bove, G., Vecchio, G. M., Barbaranelli, C., \& Bandura, A. (2008). Longitudinal analysis of the role of perceived self-efficacy for self-regulated 
learning in academic continuance and achievement. Journal of educational psychology, 100(3), 525-534. https://doi.org/10.1037/0022-0663.100.3.525

Carter, R., \& Nunan, D. (2001). The Cambridge guide to teaching English to speakers of other languages. Cambridge University Press.

Casey, D., \& Murphy, K. (2009). Issues in using methodological triangulation in research. Nurse researcher, 16(4), 40-55. https://doi.org/10.7748/nr2009.07.16.4.40.c7160

Catalan, R. M. J., \& Fontecha, A. F. (2015). Lexical phrases in compositions by CLIL and non-CLIL EFL learners. In D. Marsh, M. a. L. Pérez Cañado, \& J. Ráez Padilla (Eds.), CLIL in Action: Voices from the Classroom (1 ed., pp. 82-97). Cambridge Scholars Publishing.

Cave, P. N., Evans, N. W., Dewey, D. P., \& Hartshorn, K. J. (2017). Motivational partnerships: Increasing ESL student self-efficacy. ELT Journal, 72(1), 83-96. https://doi.org/10.1093/elt/ccx027

Chapple, J. (2015). Teaching in English is not necessarily the teaching of English. International Education Studies, 8(3), 1-13. https://doi.org/10.5539/ies.v8n3p1

Chen, C.-M., Liu, H., \& Huang, H.-B. (2019). Effects of a mobile game-based English vocabulary learning app on learners' perceptions and learning performance: A case study of Taiwanese EFL learners. ReCALL (Cambridge, England), 31(2), 170-188. https://doi.org/10.1017/S0958344018000228

Chen, H., \& Boore, J. (2010). Translation and back-translation in qualitative nursing research: Methodological review. Journal Of Clinical Nursing, 19(1-2), 234-239. https://doi.org/10.1111/j.1365-2702.2009.02896.x

Chen Hsieh, J. S., Wu, W.-C. V., \& Marek, M. W. (2017). Using the flipped classroom to enhance EFL learning. Computer Assisted Language Learning, 30(1-2), 1-21.

\section{https://doi.org/10.1080/09588221.2015.1111910}

Chiu, T. K. F., \& Churchill, D. (2016). Adoption of mobile devices in teaching: Changes in teacher beliefs, attitudes and anxiety. Interactive Learning Environments, 24(2), 317-327. https://doi.org/10.1080/10494820.2015.1113709

Cho, D. W. (2012). English-medium instruction in the university context of Korea: Tradeoff between teaching outcomes and media-initiated university ranking. Journal of Asia TEFL, 9(4), 135-163.

Choi, H., Kim, J., Bang, K. S., Park, Y. H., Lee, N. J., \& Kim, C. (2015). Applying the flipped learning model to an English-medium nursing course. Journal of Korean Academy of Nursing, 45(6), 939-948. https://doi.org/10.4040/jkan.2015.45.6.939 
Choi, H. J., \& Park, J.-H. (2013). Historical analysis of the policy on the college entrance system in South Korea. International Education Studies, 6(11), 106 -121.

https://doi.org/10.5539/ies.v6n11p106

Choi, S. J. (2013). Issues and challenges in offering English-medium instruction: A close examination of the classroom experiences of professors. Studies in English Language Literature, 39(2), 275-306.

Choudhuri, D., Glauser, A., \& Peregoy, J. (2004). Guidelines for writing a qualitative manuscript for the Journal of Counseling \& Development. Journal of Counseling and Development, 82(4), 443-446. https://doi.org/10.1002/j.1556-6678.2004.tb00332.x

Chow, J., Tse, A., \& Armatas, C. (2018). Comparing trained and untrained teachers on their use of LMS tools using the Rasch analysis. Computers \& Education, 123(2018), 124-137. https://doi.org/10.1016/i.compedu.2018.04.009

Chu, T.-H., \& Chen, Y.-Y. (2016). With good we become good: Understanding e-learning adoption by theory of planned behavior and group influences. Computers \& Education, 92, 37-52. https://doi.org/10.1016/i.compedu.2015.09.013

Chuang, H.-H., Weng, C.-Y., \& Huang, F.-C. (2015). A structure equation model among factors of teachers' technology integration practice and their TPCK. Computers \& Education, 86(2015), 182-191. https://doi.org/10.1016/i.compedu.2015.03.016

Chuang, Y.-T. (2017). MEMIS: A mobile-supported English-medium instruction system. Telematics and Informatics, 34(2), 640-656. https://doi.org/10.1016/j.tele.2016.10.007

Clark-Gordon, C. V., Bowman, N. D., Hadden, A. A., \& Frisby, B. N. (2019). College instructors and the digital red pen: An exploratory study of factors influencing the adoption and nonadoption of digital written feedback technologies. Computers \& Education, 128, 414426. https://doi.org/10.1016/i.compedu.2018.10.002

Clarke, V., \& Braun, V. (2017). Thematic analysis. The Journal of Positive Psychology: Qualitative Positive Psychology., 12(3), 297-298. https://doi.org/10.1080/17439760.2016.1262613

Cobb, T. (2009). Web vocabprofile: An adaptation of Heatley \& Nation's (1994) Range.

\section{https://www.lextutor.ca}

Cooner, T. S. (2010). Creating opportunities for students in large cohorts to reflect in and on practice: Lessons learnt from a formative evaluation of students' experiences of a technology-enhanced blended learning design. British Journal of Educational Technology, 41(2), 271-286. https://doi.org/10.1111/j.1467-8535.2009.00933.x 
Cope, D. G. (2014). Methods and meanings: Credibility and trustworthiness of qualitative research. Oncology nursing forum, 41(1), 89-91.

Corbetta, P. (2003). Social research: Theory, methods and techniques. Sage.

Coşgun, G., \& Hasırcı, B. (2017). The impact of English medium instruction (EMI) on students' language abilities. International Journal of Curriculum and Instruction, 9(2), 11-20.

Cots, J. M. (2013). Introducing English-medium instruction at the University of Lleida, Spain: Intervention, beliefs and practices. In A. Doiz, D. Lasagabaster, \& J. M. Sierra (Eds.), English-medium instruction at universities: Global challenges. Multilingual Matters.

Coyle, D., Hood, P., \& Marsh, D. (2010). Content and language integrated learning. Cambrige University Press.

Crawford, R. (2017). Rethinking teaching and learning pedagogy for education in the twenty-first century: Blended learning in music education. Music Education Research, 19(2), 195-213. https://doi.org/10.1080/14613808.2016.1202223

Creese, A., \& Martin, P. (2003). Multilingual classroom ecologies: Inter-relationships, interactions and ideologies. International Journal of Bilingual Education and Bilingualism, 6(3-4), 161-167. https://doi.org/10.1080/13670050308667778

Creswell, J. W. (2018a). Qualitative inquiry \& research design : Choosing among five approaches (4th ed.). Sage Publications.

Creswell, J. W. (2018b). Research design : Qualitative, quantitative, and mixed methods approaches (4th ed.). Sage Publications.

Creswell, J. W., Hanson, W. E., Plano, V. L. C., \& Morales, A. (2007). Qualitative research designs selection and implementation. The counseling psychologist, 35(2), 236-264. https://doi.org/10.1177/0011000006287390

Cubeles, A., \& Riu, D. (2016). Teachers' use of technology in the university classroom. The Fourth International Conference on technological ecosystems for enhancing multiculturality, Spain.

Czerniewicz, L., \& Brown, C. (2009). A study of the relationship between institutional policy, organisational culture and e-learning use in four South African universities. Computers \& Education, 53(1), 121-131. https://doi.org/10.1016/j.compedu.2009.01.006

Dafouz, E. (2018). English-medium instruction and teacher education programmes in higher education: Ideological forces and imagined identities at work. International Journal of Bilingual Education Bilingualism, 21(5), 540-552.

https://doi.org/10.1080/13670050.2018.1487926 
Dafouz, E., Camacho, M., \& Urquia, E. (2014). 'Surely they can't do as well': A comparison of business students' academic performance in English-medium and Spanish-as-firstlanguage-medium programmes. Language Education, 28(3), 223-236.

\section{https://doi.org/10.1080/09500782.2013.808661}

Dafouz, E., Hüttner, J., \& Smit, U. (2016). University teachers' beliefs of language and content integration in English-medium education in multilingual university settings. In T. Nikula, E. Dafouz, P. Moore, \& U. Smit (Eds.), Conceptualising integration in CLIL multilingual education (pp. 123-143). Multilingual Matters.

Dafouz, E., \& Smit, U. (2016). Towards a dynamic conceptual framework for English-medium education in multilingual university settings. Applied Linguistics, 37(3), 397-415. https://doi.org/10.1093/applin/amu034

Dafouz, E., \& Smit, U. (2020). ROAD-MAPPING English medium education in the internationalised university. Springer International Publishing. https://doi.org/10.1007/978-3-030-23463-8

Dallinger, S., Jonkmann, K., Hollm, J., \& Fiege, C. (2016). The effect of content and language integrated learning on students' English and history competences-Killing two birds with one stone? Learning and Instruction, 41, 23-31.

Dalton-Puffer, C. (2008). Outcomes and processes in content and language integrated learning (CLIL): Current research from Europe. Heidelberg: Carl Winter.

Dalton-Puffer, C. (2011). Content-and-language integrated learning: From practice to principles? Annual Review of applied linguistics, 31(2011), 182-204.

https://doi.org/10.1017/S0267190511000092

Dalton-Puffer, C., Nikula, T., \& Smit, U. (2010). Language use and language learning in CLIL classrooms (Vol. 7). John Benjamins Publishing.

Dang, T. H., \& Moskovsky, C. (2019). English-medium instruction in Vietnamese higher education: A ROAD-MAPPING perspective. Issues in Educational Research, 29(4), 13191336.

Dang, T. K. A., Nguyen, H. T. M., \& Le, T. T. T. (2013). The impacts of globalisation on EFL teacher education through English as a medium of instruction: An example from Vietnam. Current Issues in Language Planning, 14(1), 52-72. https://doi.org/10.1080/14664208.2013.780321

Daniela Dal Forno, K., Cristiane Cardoso de, P., Stela Maris de Mello, P., Eliane Tatsch, N., Raquel Einloft, K., \& Laura Ferreira, C. (2017). Focus group on qualitative research: Experience 
report. Revista Brasileira de Enfermagem, 70(2), 424-429. https://doi.org/10.1590/00347167-2016-0091

Darling-Hammond, L., Hammerness, K., Grossman, P., Rust, F., \& Shulman, L. S. (2005). The design of teacher education programs. In L. Darling-Hammond \& J. Bransford (Eds.), Preparing teachers for a changing world: What teachers should learn be able to do (pp. 390-441). Jossey-Bass.

Day, C., \& Kington, A. (2008). Identity, well-being and effectiveness: The emotional contexts of teaching. Pedagogy, culture \& society, 16(1), 7-23.

\section{https://doi.org/10.1080/14681360701877743}

de Wit, H., \& Hunter, F. (2015). The future of internationalization of higher education in Europe. International higher education(83), 2-3. https://doi.org/10.6017/ihe.2015.83.9073

Dearden, J. (2014). English as a medium of instruction-a growing global phenomenon. British Council.

Dearden, J., \& Macaro, E. (2016). Higher education teachers' attitudes towards English medium instruction: A three-country comparison. Studies in Second Language Learning Teaching, 6(3), 455-486. https://doi.org/10.14746/sllt.2016.6.3.5

Delgado-Márquez, B. L., Escudero-Torres, M. A., \& Hurtado-Torres, N. E. (2013). Being highly internationalised strengthens your reputation: An empirical investigation of top higher education institutions. Higher Education, 66(5), 1-15. https://doi.org/10.1007/s10734$\underline{013-9626-8}$

Deng, F., Chai, C. S., Tsai, C.-C., \& Lee, M.-H. (2014). The relationships among Chinese practicing teachers' epistemic beliefs, pedagogical beliefs and their beliefs about the use of ICT. Journal of Educational Technology \& Society, 17(2), 245-256.

Denzin, N. K., \& Lincoln, Y. S. (2013). The landscape of qualitative research (4th ed.). Sage Publications.

Denzin, N. K., \& Lincoln, Y. S. (2018). The Sage handbook of qualitative research (5th ed.). Sage Publications.

Do, H. M., \& Do, Q. T. N. (2014). Higher and tertiary education in Vietnam. In R. King, J. Lee, S. Marginson, \& R. Naidoo (Eds.), Higher Education in Vietnam (pp. 29-53). Springer. https://doi.org/10.1057/9781137436481 2

Doiz, A., \& Lasagabaster, D. (2017). Management teams and teaching staff: do they share the same beliefs about obligatory CLIL programmes and the use of the L1? Language and Education, 31(2), 93-109. https://doi.org/10.1080/09500782.2017.1290102 
Doiz, A., Lasagabaster, D., \& Sierra, J. M. (2011). Internationalisation, multilingualism and English-medium instruction. World Englishes, 30(3), 345-359.

https://doi.org/10.1111/j.1467-971X.2011.01718.x

Dovemark, M. (2010). Teachers' collective actions, alliances and resistance within neo-liberal ideas of education: The example of the individual programme. European Educational Research Journal, 9(2), 232-244. https://doi.org/10.2304/eerj.2010.9.2.232

Eickelmann, B. (2011). Supportive and hindering factors to a sustainable implementation of ICT in schools. Journal for Educational Research Online, 3(1), 75-103.

Englund, C., Olofsson, A. D., \& Price, L. (2017). Teaching with technology in higher education: Understanding conceptual change and development in practice. Higher Education Research \& Development, 36(1), 73-87. https://doi.org/10.1080/07294360.2016.1171300

Ertmer, P. A., \& Ottenbreit-Leftwich, A. T. (2010). Teacher technology change: How knowledge, confidence, beliefs, and culture intersect. Journal of Research on Technology in Education, 42(3), 255-284. https://doi.org/10.1080/15391523.2010.10782551

Ertmer, P. A., Ottenbreit-Leftwich, A. T., \& Tondeur, J. (2014). Teachers' beliefs and uses of technology to support 21st-century teaching and learning. In H. Fives \& M. G. Gill (Eds.), International handbook of research on teacher beliefs (pp. 403-418). Routledge.

Evans, S., \& Morrison, B. (2011). Meeting the challenges of English-medium higher education: The first-year experience in Hong Kong. English for Specific Purposes, 30(3), 198-208. https://doi.org/10.1016/i.esp.2011.01.001

Evans, T. L. (2014). Teaching and learning with digital technologies in the intermediate school classroom : An Activity Theory analysis of classroom interactions [Doctoral dissertation]. Victoria University of Wellington.

Farah, A. (2012). Factors influencing teachers' technology self-efficacy: A case study (Publication Number 3494891) [Doctoral thesis, Liberty University]. ProQuest Dissertations \& Theses Global.

Farley, H., Murphy, A., Johnson, C., Carter, B., Lane, M., Midgley, W., Hafeez-Baig, A., Dekeyser, S., \& Koronios, A. (2015). How do students use their mobile devices to support learning? A case study from an Australian regional university. Journal of interactive media in education, 2015(1), 13. https://doi.org/10.5334/jime.ar

Feast, V. (2002). The impact of IELTS scores on performance at university. International Education Journal, 3(4), 70-85. 
Fernández-Fontecha, A., O’Halloran, K. L., Wignell, P., \& Tan, S. (2020). Scaffolding CLIL in the science classroom via visual thinking: A systemic functional multimodal approach. Linguistics and Education, 55, 1-10. https://doi.org/10.1016/j.linged.2019.100788

Ferrer-Torregrosa, J., Torralba, J., Jimenez, M., García, S., \& Barcia, J. (2015). ARBOOK:

Development and assessment of a tool based on augmented reality for anatomy. Journal of Science Education and Technology, 24(1), 119-124. https://doi.org/10.1007/s10956$\underline{014-9526-4}$

Fisher, R., Perényi, Á., \& Birdthistle, N. (2018). The positive relationship between flipped and blended learning and student engagement, performance and satisfaction. Active Learning in Higher Education(2018), 1-17. https://doi.org/10.1177/1469787418801702

Forey, G., \& Polias, J. (2017). Multi-semiotic resources providing maximal input in teaching science through English. In A. Llinares \& T. Morton (Eds.), Applied linguistics perspectives on CLIL (pp. 145-164). John Benjamins Publishing Company.

\section{https://doi.org/10.1075/IIlt.47}

Forman, R. (2014). How local teachers respond to the culture and language of a global English as a foreign language textbook. Language, Culture and Curriculum, 27(1), 72-88. https://doi.org/10.1080/07908318.2013.868473

Frankenberg-Garcia, A., Lew, R., Roberts, J. C., Rees, G. P., \& Sharma, N. (2019). Developing a writing assistant to help EAP writers with collocations in real time. 31(1), 23-39.

\section{https://doi.org/10.1017/S0958344018000150}

Froment, F., García González, A. J., \& Bohórquez, M. R. (2017). The use of social networks as a communication tool between teachers and students: A literature review. Turkish Online Journal of Educational Technology, 16(4), 126-144.

Fu, G., \& Clarke, A. (2019). Individual and collective agencies in China's curriculum reform: A case of physics teachers. Journal of Research in Science Teaching, 56(1), 45-63.

https://doi.org/10.1002/tea.21467

Fullan, M., \& Langworthy, M. (2013). Towards a new end: New pedagogies for deep learning. Seattle, Washington: Collaborative Impact.

Galvis, H. A. (2012). Understanding beliefs, teachers' beliefs and their impact on the use of computer technology (Hacia la compresión de las creencias, convicciones del educador y su impacto en el uso de la tecnología). Profile Issues in Teachers' Professional Development, 14(2), 95-112. 
Gao, X. A. (2010). Strategic language learning: The roles of agency and context. Multilingual Matters.

García, O. (2009). Bilingual education in the 21st century : A global perspective. Wiley-Blackwell Publications.

García, O., \& Lin, A. (2019). Translanguaging in Bilingual Education. In O. García, A. Lin, \& S. May (Eds.), Bilingual and Multilingual Education. Encyclopedia of Language and Education. (pp. 117-130). Springer International Publishing. https://doi.org/10.1007/978-3-319$\underline{02324-3}$

Garrote Jurado, R., Pettersson, T., Regueiro Gomez, A., \& Scheja, M. (2014). Classification of the features in learning management systems.

Gee, J. P. (2014). Social linguistics and literacies : Ideology in discourses (5th ed.). Routledge.

Ghorbani, M. R., \& Alavi, S. Z. (2014). Feasibility of adopting English-medium instruction at Iranian universities. Current Issues in Education, 17(1), 1-17.

Gierlinger, E. (2015). 'You can speak German, sir': on the complexity of teachers' L1 use in CLIL. Language and Education, 29(4), 347-368.

https://doi.org/10.1080/09500782.2015.1023733

Gimeno, A., Seiz, R., Siqueira, J. M. d., \& Martínez, A. (2010). Content and language integrated learning in higher technical education using the inGenio online multimedia authoring tool. Procedia - Social and Behavioral Sciences, 2(2), 3170-3174.

https://doi.org/10.1016/j.sbspro.2010.03.484

Gleeson, M. (2010). Language and content? : how do curriculum teachers of year 12 English language learners combine the two disciplines? [Doctoral dissertation]. Victoria University of Wellington.

Gleeson, M. (2015). 'It's the nature of the subject': Secondary teachers' disciplinary beliefs and decisions about teaching academic language in their content classes. Australian Journal of Language and Literacy, The, 38(2), 104.

Gleeson, M., \& Davison, C. (2016). A conflict between experience and professional learning: Subject teachers' beliefs about teaching English language learners. Relc Journal, 47(1), 43-57. https://doi.org/10.1177/0033688216631221

Golden, N. A. (2018). Narrating neoliberalism: Alternative education teachers' conceptions of their changing roles. Teaching Education, 29(1), 1-16. 
Golden, N. A., \& Womack, E. (2016). Cultivating literacy and relationships with adolescent scholars of color. The English Journal, 105(3), 36-42.

Golonka, E. M., Bowles, A. R., Frank, V. M., Richardson, D. L., \& Freynik, S. (2014). Technologies for foreign language learning: A review of technology types and their effectiveness. Computer Assisted Language Learning, 27(1), 70-105. https://doi.org/10.1080/09588221.2012.700315

Green, J. C., Aziz, T., Joseph, J., Ravanam, A., Shahab, S., \& Straus, L. (2018). YouTube enhanced case teaching in health management and policy. Health Professions Education, 4(1), 4858. https://doi.org/10.1016/j.hpe.2017.02.006

Griffin, D. K., Mitchell, D., \& Thompson, S. J. (2009). Podcasting by synchronising PowerPoint and voice: What are the pedagogical benefits? Computers \& Education, 53(2), 532-539. https://doi.org/10.1016/i.compedu.2009.03.011

Guba, E. G. (1981). Criteria for assessing the trustworthiness of naturalistic inquiries. ECTJ, 29(2), 75-91. https://doi.org/10.1007/BF02766777

Guest, G., Namey, E., Taylor, J., Eley, N., \& McKenna, K. (2017). Comparing focus groups and individual interviews: Findings from a randomized study. International Journal of Social Research Methodology, 20(6), 693-708.

https://doi.org/10.1080/13645579.2017.1281601

Gupta, M., \& Dharamveer. (2017). Prospective teacher's attitude towards the use of ICT: A comparative study between C.C.S. University, Meerut and Kurukshetra University, Kurukshetra. Educational Quest: An International Journal of Education and Applied Social Sciences, 8(1), 81-88. https://doi.org/10.5958/2230-7311.2017.00014.9

Hajer, M. (2000). Creating a language-promoting classroom: Content-area teachers at work. In J. K. Hall \& L. S. Verplaetse (Eds.), Second and foreign language learning through classroom interaction (pp. 265-286). Routledge.

Hallinger, P. (2010). Making education reform happen: Is there an 'Asian' way? School leadership and management, 30(5), 401-418. https://doi.org/10.1080/13632434.2010.502524

Hamid, M. O., Jahan, I., \& Islam, M. M. (2013). Medium of instruction policies and language practices, ideologies and institutional divides: Voices of teachers and students in a private university in Bangladesh. Current Issues in Language Planning, 14(1), 144-163. https://doi.org/10.1080/14664208.2013.771417

Hamilton-Ekeke, J., \& Mbachu, C. (2015). The place of information, communication and technology (ICT) in teaching and learning in Nigerian tertiary institutions. American 
Journal of Educational Research, 3(3), 340-347. https://doi.org/10.12691/education-3-3 $-13$

Hammond, J., \& Gibbons, P. (2005). What is scaffolding. Teachers' voices, 8(2005), 8-16. Hannafin, M., Hannafin, K., \& Gabbitas, B. (2009). Re-examining cognition during studentcentered, Web-based learning. Educational Technology Research and Development, 57(6), 767-785. https://doi.org/10.1007/s11423-009-9117-x

Harman, K., \& Nguyen, B. T. N. (2010). Reforming teaching and learning in Vietnam's higher education system. In G. Harman, M. Hayden, \& T. N. Pham (Eds.), Reforming higher education in Vietnam (pp. 65-86). Springer. https://doi.org/10.1007/978-90-481-3694-0

Harris, J., Mishra, P., \& Koehler, M. (2009). Teachers' technological pedagogical content knowledge and learning activity types: Curriculum-based technology integration reframed. Journal of Research on Technology in Education, 41(4), 393-416. https://doi.org/10.1080/15391523.2009.10782536

Harskamp, A., \& House, N. (2019). Interviews in qualitative research. Educational Psychology in Practice, 35(4), 440-441. https://doi.org/10.1080/02667363.2019.1625240

Harvey, M., Coulson, D., \& McMaugh, A. (2016). Towards a theory of the ecology of reflection: Reflective practice for experiential learning in higher education. Journal of University Teaching \& Learning Practice, 13(2), 2-24.

https://doi.org/http://ro.uow.edu.au/jutlp/vol13/iss2/2

Hashim, A., \& Low, E. L. (2014). Introduction: English in Southeast Asia. World Englishes, 33(4), 423-425. https://doi.org/10.1111/weng.12104

Hassani, K., Nahvi, A., \& Ahmadi, A. (2016). Design and implementation of an intelligent virtual environment for improving speaking and listening skills. Interactive Learning Environments, 24(1), 252-271. https://doi.org/10.1080/10494820.2013.846265

Hatlevik, O. E., Guðmundsdóttir, G. B., \& Loi, M. h. (2015). Examining factors predicting students' digital competence. Journal of Information Technology Education: Research, 14(14), 123-137.

Heitink, M., Voogt, J., Verplanken, L., van Braak, J., \& Fisser, P. (2016). Teachers' professional reasoning about their pedagogical use of technology. Computers education, 101(2016), 70-83. https://doi.org/10.1016/i.compedu.2016.05.009

Heller, M. (2008). Language and the nation-state: Challenges to sociolinguistic theory and practice. Journal of Sociolinguistics, 12(4), 504-524. https://doi.org/10.1111/j.1467$\underline{9841.2008 .00373 . x}$ 
Henderson, M., Selwyn, N., \& Aston, R. (2017). What works and why? Student perceptions of 'useful' digital technology in university teaching and learning. Studies in higher education, 42(8), 1567-1579. https://doi.org/10.1080/03075079.2015.1007946

Henderson, M., Selwyn, N., Finger, G., \& Aston, R. (2015). Students' everyday engagement with digital technology in university: Exploring patterns of use and 'usefulness'. Journal of Higher Education Policy and Management, 37(3), 308-319.

https://doi.org/10.1080/1360080X.2015.1034424

Hengsadeekul, C., Koul, R., \& Kaewkuekool, S. (2014). Motivational orientation and preference for English-medium programs in Thailand. International Journal of Educational Research, 66(2014), 35-44. https://doi.org/10.1016/j.ijer.2014.02.001

Hennink, M., Hutter, I., \& Bailey, A. (2020). Qualitative research methods (2nd ed.). Sage Publications

Hofstede, G. (2001). Culture's consequences: Comparing values, behaviors, institutions and organizations across nations. Sage publications.

Hoic-Bozic, N., Holenko Dlab, M., \& Mornar, V. (2016). Recommender system and Web 2.0 tools to enhance a blended learning model. IEEE Transactions on Education, 59(1), 39-44. https://doi.org/10.1109/TE.2015.2427116

Horton, J., Macve, R., \& Struyven, G. (2004). Qualitative research: Experiences in using semistructured interviews. In C. Humphrey \& B. Lee (Eds.), The real life guide to accounting research (pp. 339-357). Elsevier. https://doi.org/10.1016/B978-008043972-3/50022-0

Houghton, C., Casey, D., Shaw, D., \& Murphy, K. (2013). Rigour in qualitative case-study research. Nurse researcher, 20(4), 12-17.

https://doi.org/10.7748/nr2013.03.20.4.12.e326

Hove, S. E., \& Anda, B. (2005). Experiences from conducting semi-structured interviews in empirical software engineering research. 11th IEEE International Software Metrics Symposium Como, Italy.

Hsieh, C. N. (2017). The case of Taiwan: Perceptions of college students about the use of the TOEIC $^{\circledR}$ tests as a condition of graduation. ETS Research Report Series, 2017(1), 1-12. https://doi.org/10.1002/ets2.12179

Hsu, L. (2016). Examining EFL teachers' technological pedagogical content knowledge and the adoption of mobile-assisted language learning: A partial least square approach. Computer Assisted Language Learning, 29(8), 1287-1297. https://doi.org/10.1080/09588221.2016.1278024 
Hu, G., \& Lei, J. (2014). English-medium instruction in Chinese higher education: A case study. Higher Education, 67(5), 551-567. https://doi.org/10.1007/s10734-013-9661-5

Hu, G., Li, L., \& Lei, J. (2014). English-medium instruction at a Chinese University: Rhetoric and reality. Language Policy, 13(1), 21-40. https://doi.org/10.1007/s10993-013-9298-3

Huang, D.-F. (2015). Exploring and assessing effectiveness of English medium instruction courses: The students' perspectives. Procedia-Social Behavioral Sciences, 173(2015), 7178. https://doi.org/10.1016/i.sbspro.2015.02.033

Huges, S., \& Madrid, D. (2015). The written production of CLIL. In D. Marsh, M. a. L. Pérez Cañado, \& J. Ráez Padilla (Eds.), CLIL in Action: Voices from the Classroom (pp. 98-111). Cambridge Scholars Publishing.

Hunt, K. W. (1966). Recent measures in syntactic development. Elementary English, 43(7), 732739.

Islam, M. (2013). English medium instruction in the private universities in Bangladesh. Indonesian Journal of Applied Linguistics, 3(1), 126-137. https://doi.org/10.17509/ijal.v3i1.195

Jääskelä, P., Häkkinen, P., \& Rasku-Puttonen, H. (2017). Teacher beliefs regarding learning, pedagogy, and the use of technology in higher education. Journal of Research on Technology in Education, 49(3-4), 198-211.

https://doi.org/10.1080/15391523.2017.1343691

Jensen, J. (2019). A systematic literature review of the use of semantic web technologies in formal education. British Journal of Educational Technology, 50(2), 505-517. https://doi.org/10.1111/bjet.12570

Jexenflicker, S., \& Dalton-Puffer, C. (2010). Comparing the writing of CLIL and non-CLIL students in higher colleges of technology. In C. Dalton-Puffer, T. Nikula, \& U. Smit (Eds.), Language use language learning in CLIL classrooms (pp. 169-190). John Benjamins Publishing.

Jiang, A. L., \& Zhang, L. J. (2019). Chinese students' perceptions of English learning affordances and their agency in an English-medium instruction classroom context. Language and Education, 33(4), 322-339. https://doi.org/10.1080/09500782.2019.1578789

Jiang, H. (2016). Revisiting individualism and collectivism: A multinational examination of preservice teachers' perceptions on student academic performances. Intercultural Education, 27(1), 101-110. https://doi.org/10.1080/14675986.2016.1144327 
Joe, Y., \& Lee, H.-K. (2013). Does English-medium instruction benefit students in EFL contexts? A case study of medical students in Korea. The Asia-Pacific Education Researcher, 22(2), 201-207. https://doi.org/10.1007/s40299-012-0003-7

Joo, Y. J., Park, S., \& Lim, E. (2018). Factors influencing preservice teachers' intention to use technology: TPACK, teacher self-efficacy, and technology acceptance model. Educational Technology \& Society, 21(3), 48.

Jung, I., \& Lee, Y. (2015). YouTube acceptance by university educators and students: A crosscultural perspective. Innovations in Education and Teaching International, 52(3), 243253. https://doi.org/10.1080/14703297.2013.805986

Kajornboon, A. B. (2005). Using interviews as research instruments. E-journal for Research Teachers, 2(1), 1-9.

Kalolo, J. (2019). Digital revolution and its impact on education systems in developing countries. Education and Information Technologies, 24(1), 345-358. https://doi.org/10.1007/s10639-018-9778-3

Kang, S., \& Park, H. (2005). English as the medium of instruction in Korean engineering education. Korean Journal of Applied Linguistics, 21(1), 155-174.

Karabassova, L. (2018). Teachers' conceptualization of content and language integrated learning (CLIL): Evidence from a trilingual context. International Journal of Bilingual Education and Bilingualism(2018), 1-13. https://doi.org/10.1080/13670050.2018.1550048

Karjanto, N., \& Simon, L. (2018). English-medium instruction calculus: Is flipping helpful? arXiv.org. https://arxiv.org/pdf/1611.08377.pdf

Karmani, S. (2010). On perceptions of the socialising effects of English-medium education on students at a Gulf Arab University with particular reference to the United Arab Emirates (Publication Number U570003) [Doctoral dissertation, University of Exeter]. ProQuest Dissertations \& Theses Global.

Kennewell, S., Tanner, H., Jones, S., \& Beauchamp, G. (2008). Analysing the use of interactive technology to implement interactive teaching. Journal of Computer assisted learning, 24(1), 61-73. https://doi.org/10.1111/j.1365-2729.2007.00244.x

Kılıçkaya, F. (2015). Computer-based grammar instruction in an EFL context: Improving the effectiveness of teaching adverbial clauses. Computer Assisted Language Learning, 28(4), 325-340. https://doi.org/10.1080/09588221.2013.818563 
Kim, C., Kim, M. K., Lee, C., Spector, J. M., \& DeMeester, K. (2013). Teacher beliefs and technology integration. Teaching teacher education, 29, 76-85. https://doi.org/10.1016/j.tate.2012.08.005

Kim, E. G., \& Shin, A. (2014). Seeking an effective program to improve communication skills of non-English-speaking graduate Engineering students: The case of a Korean Engineering school. IEEE Transactions on Professional Communication, 57(1), 41-55.

https://doi.org/10.1109/TPC.2014.2310784

Kim, P., Hagashi, T., Carillo, L., Gonzales, I., Makany, T., Lee, B., \& Gàrate, A. (2011).

Socioeconomic strata, mobile technology, and education: a comparative analysis.

Educational Technology Research and Development, 59(4), 465-486.

https://doi.org/10.1007/s11423-010-9172-3

King, A. (2000). The accidental derogation of the lay actor: A critique of Giddens's concept of structure. Philosophy of the Social Sciences, 30(3), 362-383.

https://doi.org/10.1177/004839310003000302

Kirkpatrick, A. (2011). English as an Asian lingua franca and the multilingual model of ELT. Language Teaching, 44(2), 212-224. https://doi.org/10.1017/S0261444810000145

Kirkwood, A., \& Price, L. (2012). The influence upon design of differing conceptions of teaching and learning with technology. In A. D. Olofsson \& J. O. Lindberg (Eds.), Informed design of educational technologies in higher education: Enhanced learning and teaching (pp. 120). IGI Global.

Kirkwood, A., \& Price, L. (2014). Technology-enhanced learning and teaching in higher education: What is 'enhanced' and how do we know? A critical literature review. Learning, Media and Technology, 39(1), 6-36.

\section{https://doi.org/10.1080/17439884.2013.770404}

Klemenčič, M. (2015). What is student agency? An ontological exploration in the context of research on student engagement. In M. Klemenčič, S. Bergan, \& R. Primožič (Eds.), Student engagement in Europe: Society, higher education and student governance (pp. 11-29). Council of Europe Publishing.

Klemenčič, M. (2017). From student engagement to student agency: Conceptual considerations of European policies on student-centered learning in higher education. Higher Education Policy, 30(1), 69-85. 
Koh, J. (2019). Four pedagogical dimensions for understanding flipped classroom practices in higher education: A systematic review. Kuram ve Uygulamada Egitim Bilimleri, 19(4), 1433. https://doi.org/10.12738/estp.2019.4.002

Komori-Glatz, M. F. (2017). English as a business lingua franca in multicultural student teamwork [Unpublished doctoral thesis]. University of Vienne.

Kongsuebchart, J., \& Suppasetseree, S. (2018). The effect of a weblog-based electronic portfolio on Thai EFL undergraduate students' English writing skills. Computer Assisted Language Learning Electronic Journal, 19(2), 28-46.

Köroglu, Z. Ç., \& Çakir, A. (2017). Implementation of flipped instruction in language classrooms: An alternative way to develop speaking skills of pre-service English language teachers. International Journal of Education and Development using Information and Communication Technology, 13(2), 42-55.

Krashen, S. D. (1985). The input hypothesis: Issues and implications. Addison-Wesley Longman Krefting, L. (1991). Rigor in qualitative research: The assessment of trustworthiness. American journal of occupational therapy, 45(3), 214-222. https://doi.org/10.5014/ajot.45.3.214

Kreijns, K., Van Acker, F., Vermeulen, M., \& van Buuren, H. (2013). What stimulates teachers to integrate ICT in their pedagogical practices? The use of digital learning materials in education. Computers in Human Behavior, 29(1), 217-225.

\section{https://doi.org/10.1016/j.chb.2012.08.008}

Krueger, R. A. (2014). Focus groups: A practical guide for applied research. Sage publications.

Lai, K. W., \& Hong, K. S. (2015). Technology use and learning characteristics of students in higher education: Do generational differences exist? British Journal of Educational Technology, 46(4), 725-738. https://doi.org/10.1111/bjet.12161

Larsen, M. A. (2016). Globalisation and internationalisation of teacher education: A comparative case study of Canada and Greater China. Teaching Education, 27(4), 396-409.

https://doi.org/10.1080/10476210.2016.1163331

Lasagabaster, D. (2011). English achievement and student motivation in CLIL and EFL settings. Innovation in language Learning Teaching, 5(1), 3-18.

\section{https://doi.org/10.1080/17501229.2010.519030}

Lasagabaster, D. (2017). The impact of the spread of English-medium instruction on Spanish universities. Language Teaching, 50(2017), 11-18. 
Lázaro, A. (2012). Faster and further morphosyntactic development of CLIL vs. EFL BasqueSpanish bilinguals learning English in high-school. International Journal of English Studies, 12(1), 79-96. https://doi.org/10.6018/ijes.12.1.125621

Le, D. M. (2012). English as a medium of instruction at tertiary education system in Vietnam. Journal of Asia TEFL, 9(2), 97-122.

Leask, B. (2015). Internationalizing the curriculum. Routledge.

Lee, E., \& Hannafin, M. (2016). A design framework for enhancing engagement in studentcentered learning: Own it, learn it, and share it. Educational Technology Research and Development, 64(4), 707-734. https://doi.org/10.1007/s11423-015-9422-5

Lei, J., \& Hu, G. (2014). Is English-medium instruction effective in improving Chinese undergraduate students' English competence? International Review of Applied Linguistics in Language Teaching, 52(2), 99-126. https://doi.org/10.1515/iral-2014-0005

Li, Y., Garza, V., Keicher, A., \& Popov, V. (2019). Predicting high school teacher use of technology: Pedagogical beliefs, technological beliefs and attitudes, and teacher training. Technology, Knowledge and Learning, 24(3), 501-518. https://doi.org/10.1007/s10758-018-9355-2

Lin, L. H., \& Morrison, B. (2010). The impact of the medium of instruction in Hong Kong secondary schools on tertiary students' vocabulary. Journal of English for Academic Purposes, 9(4), 255-266. https://doi.org/10.1016/i.jeap.2010.09.002

Lincoln, Y. S., \& Guba, E. G. (2007). Naturalistic inquiry. The Blackwell encyclopedia of sociology. https://doi.org/10.1002/9781405165518.wbeosn006

Linnenbrink, E. A., \& Pintrich, P. R. (2003). The role of self-efficacy beliefs instudent engagement and learning in the classroom. Reading Writing Quarterly, 19(2), 119-137.

\section{https://doi.org/10.1080/10573560308223}

Liping, P. S., Pirkko, S., \& Heli, R. (2018). How to trigger students' interest in digital learning environments: A systematic literature review. Seminar.net, 14(1), 62-84.

Liu, H., Lin, C.-H., \& Zhang, D. (2017). Pedagogical beliefs and attitudes toward information and communication technology: A survey of teachers of English as a foreign language in China. Computer Assisted Language Learning, 30(8), 745-765.

\section{https://doi.org/10.1080/09588221.2017.1347572}

Liu, M.-H. (2016). Blending a class video blog to optimize student learning outcomes in higher education. The Internet and Higher Education, 30(2016), 44-53.

https://doi.org/10.1016/j.iheduc.2016.03.001 
Liu, Y., \& Fang, F. (2020). Translanguaging Theory and Practice: How Stakeholders Perceive Translanguaging as a Practical Theory of Language. Relc Journal(2020), 1-9. https://doi.org/10.1177/0033688220939222

Loewenberg-Ball, D., Thames, M. H., \& Phelps, G. (2008). Content knowledge for teaching: What makes it special. Journal of teacher education, 59(5), 389-407.

\section{https://doi.org/10.1177/0022487108324554}

Longhurst, R. (2009). Interviews: In-depth, semi-structured. In R. Kitchin \& N. Thrift (Eds.), International Encyclopedia of Human Geography (pp. 580-584). Elsevier Science.

Lötter, M. J., \& Jacobs, L. (2020). Using smartphones as a social constructivist pedagogical tool for inquiry-supported problem-solving: An exploratory study. Journal of Teaching in Travel and Tourism. https://doi.org/10.1080/15313220.2020.1715323

Lowenberg, P. H. (2002). Assessing English proficiency in the expanding circle. World Englishes, 21(3), 431-435.

Ly, T. H., \& Habibah, A. J. (2013). Attitudes towards ICT integration into curriculum and usage among university lecturers in Vietnam. International Journal of Instruction, 6(2), 53-66.

Macaro, E., \& Akincioglu, M. (2018). Turkish university students' perceptions about Englishmedium instruction: Exploring year group, gender and university type as variables. Journal of Multilingual Multicultural Development, 39(3), 256-270.

\section{https://doi.org/10.1080/01434632.2017.1367398}

Macaro, E., Curle, S., Pun, J., An, J., \& Dearden, J. (2018). A systematic review of English medium instruction in higher education. Language Teaching, 51(1), 36-76.

https://doi.org/10.1017/S0261444817000350

MacBlain, S. (2018). Albert Bandura and social learning theory. In S. MacBlain (Ed.), Learning Theories For Early Years Practice (1st ed., pp. 63-65). Sage Publications.

Mackey, J. (2015). Leading change with digital technologies in education. SET Tesearch Information for Teachers(2), 17-25. https://doi.org/10.18296/set.0014

Mackinnon, A., \& Le, H. V. (2014). The sociocultural context of higher education in Vietnam: A case for collaborative learning in physics courses. International journal of educational studies, 1(3), 145-161.

Maeng, J. L., Mulvey, B. K., Smetana, L. K., \& Bell, R. L. (2013). Preservice teachers' TPACK: Using technology to support inquiry instruction. Journal of Science Education and Technology, 22(6), 838-857. https://doi.org/10.1007/s10956-013-9434-z 
Mahan, K. R. (2020). The comprehending teacher: Scaffolding in content and language integrated learning (CLIL). The Language Learning Journal(2020), 1-15.

Mahan, K. R., Brevik, L. M., \& Ødegaard, M. (2018). Characterizing CLIL teaching: New insights from a lower secondary classroom. International Journal of Bilingual Education and Bilingualism(2018), 1-18. https://doi.org/10.1080/13670050.2018.1472206

Maier, C., Laumer, S., Eckhardt, A., \& Weitzel, T. (2015). Giving too much social support: Social overload on social networking sites. European Journal of Information Systems, 24(5), 447-464. https://doi.org/10.1057/ejis.2014.3

Manca, S., \& Ranieri, M. (2016). Facebook and the others. Potentials and obstacles of social media for teaching in higher education. Computers \& Education, 95(2016), 216-230. https://doi.org/10.1016/j.compedu.2016.01.012

Mangan, A. (2011). Moving towards student-centred learning: A case study. The International Conference on English Pedagogy, Dublin, Ireland.

Marcelo, C., \& Yot-Domínguez, C. (2019). From chalk to keyboard in higher education classrooms: Changes and coherence when integrating technological knowledge into pedagogical content knowledge. Journal of Further and Higher Education, 43(7), 975988. https://doi.org/10.1080/0309877X.2018.1429584

Marcelo, C., Yot, C., \& Mayor, C. (2015). University teaching with digital technologies. Comunicar, 23(45), 117-124. https://doi.org/10.3916/C45-2015-12

Marchetti, L., \& Cullen, P. (2016). A multimodal approach in the classroom for creative learning and teaching. CASALC Review, Czech and Slovak Association of Language Centres, 41(2016), 39-51.

Margaryan, A., Littlejohn, A., \& Vojt, G. (2011). Are digital natives a myth or reality? University students' use of digital technologies. Computers \& Education, 56(2), 429-440. https://doi.org/10.1016/i.compedu.2010.09.004

Mascolo, M. E., \& Li, J. E. (2004). Culture and developing selves: Beyond dichotomization. JosseyBass.

Matt, G., \& Orna, O. b. (2017). Academic engagement and technology: Revisiting the technological, pedagogical and content knowledge framework (TPACK) in higher education (HE): The academics' perspectives. IAFOR Journal of Education, 5(2017), 133159. https://doi.org/10.22492/ije.5.si.06 
McIntosh, M. J., \& Morse, J. M. (2015). Situating and constructing diversity in semi-structured interviews. Global qualitative nursing research, 2(2015), 1-12.

https://doi.org/10.1177/2333393615597674

McKnight, K., O'Malley, K., Ruzic, R., Horsley, M. K., Franey, J. J., \& Bassett, K. (2016). Teaching in a digital age: How educators use technology to improve student learning. Journal of Research on Technology in Education, 48(3), 194-211. https://doi.org/10.1080/15391523.2016.1175856

Méndez García, M. D. C., \& Pavón Vázquez, V. (2012). Investigating the coexistence of the mother tongue and the foreign language through teacher collaboration in CLIL contexts: Perceptions and practice of the teachers involved in the plurilingual programme in Andalusia. International Journal of Bilingual Education and Bilingualism, 15(5), 573-592. https://doi.org/10.1080/13670050.2012.670195

Merriam, S. B. (2010). Qualitative case studies. In P. Peterson, E. Baker, \& B. McGaw (Eds.), International Encyclopedia of Education (pp. 456 - 462). Elsevier Science. https://doi.org/http://dx.doi.org/10.1016/B978-0-08-044894-7.01532-3

Merriam, S. B. (2014). Qualitative research a guide to design and implementation. Jossey Bass Ltd.

Miles, M. B. (2014). Qualitative data analysis : A methods sourcebook (3rd ed.). Sage Publications.

Miles, M. B., Huberman, A. M., \& Saldana, J. (2013). Qualitative data analysis: A methods sourcebook. Sage Publications.

Miranda, H. P., \& Russell, M. (2012). Understanding factors associated with teacher-directed student use of technology in elementary classrooms: A structural equation modeling approach. British Journal of Educational Technology, 43(4), 652-666.

https://doi.org/10.1111/j.1467-8535.2011.01228.x

Mishra, P., \& Koehler, M. J. (2006). Technological pedagogical content knowledge: A framework for teacher knowledge. Teachers College Record, 108(6), 1017-1054.

MOET. (2014). Circular No. 23/2014/TT-BGDET on "Regulations on high-quality programs in universities". Ministry of Education and Training.

Morita, N. (2004). Negotiating participation and identity in second language academic communities. TESOL Quarterly, 38(4), 573-603. https://doi.org/10.2307/3588281

Nelson, K., Courier, M., \& Joseph, G. W. (2019). An investigation of digital literacy needs of students. Journal of Information Systems Education, 22(2), 95-110. 
Neumann, D. L., \& Hood, M. (2009). The Effects of using a Wiki on student engagement and learning of report writing skills in a university statistics course. Australasian Journal of Educational Technology, 25(3), 382-398. https://doi.org/10.14742/ajet.1141

Newble, D. (1989). A handbook for teachers in universities \& colleges : A guide to improving teaching methods. Kogan Pagens.

Newman, T., \& Beetham, H. (2017). Student digital experience tracker 2017: The voice of 22,000 UK learners. Joint Information System Committee.

Ng, W. (2012). Can we teach digital natives digital literacy? Computers \& Education, 59(3), $1065-$ 1078. https://doi.org/10.1016/j.compedu.2012.04.016

Ng, W. (2015). New Digital Technology in Education Conceptualizing Professional Learning for Educators (1st ed.). Springer International Publishing. https://doi.org/10.1007/978-3319-05822-1

Ng, W., Nicholas, H., \& Williams, A. (2010). School experience influences on pre-service teachers' evolving beliefs about effective teaching. Teaching teacher education, 26(2), 278-289. https://doi.org/10.1016/j.tate.2009.03.010

Nguyen, H. (2009). Marketization of higher education in Vietnam in the era of neoliberal globalization: Policy \& practice (Publication Number MS23734) [Master's thesis, Simon Fraser University]. ProQuest Dissertations Publishing.

Nguyen, H. T. (2018). English-medium-instruction management: The missing piece in the internationalisation puzzle of Vietnamese higher education. In L. T. Tran \& S. Marginson (Eds.), Internationalisation in Vietnamese Higher Education (pp. 119-137). Springer. https://doi.org/10.1007/978-3-319-78492-2

Nguyen, H. T., Hamid, M. O., \& Moni, K. (2016). English-medium instruction and self-governance in higher education: The journey of a Vietnamese university through the institutional autonomy regime. Higher Education, 72(5), 669-683.

Nguyen, H. T., Walkinshaw, I., \& Pham, H. H. (2017). EMI programs in a Vietnamese university: Language, pedagogy and policy issues. In B. Fenton-Smith, P. Humphreys, \& I. Walkinshaw (Eds.), English medium instruction in higher education in Asia-Pacific (pp. 37-52). Springer.

Nguyen, N., \& Tran, L. T. (2018). Looking inward or outward? Vietnam higher education at the superhighway of globalization: Culture, values and changes. Journal of Asian Public Policy, 11(1), 28-45. https://doi.org/10.1080/17516234.2017.1332457 
Nguyen, Q. K., \& Nguyen, Q. C. (2008). Education in Vietnam: Development history, challenges, and solutions. An African exploration of the East Asian education experience(2008), 109154.

Niess, M. L., \& Gillow-Wiles, H. (2019). Online Instructional Strategies for Enhancing Teachers' TPACK: Experiences, Discourse, and Critical Reflection. In M. L. Niess \& H. Gillow-Wiles (Eds.), Handbook of Research on TPACK in the Digital Age (pp. 257-278). IGI Global.

Nurjannah, I., Husniyah, F., \& Harjanto, T. (2017). Teacher-Centered learning and studentcentered learning approaches in nursing school: Which one is better. Belitung Nursing Journal, 3(2), 65-72. https://doi.org/10.33546/bnj.59

Nyumba, T. O., Wilson, K., Derrick, C. J., \& Mukherjee, N. (2018). The use of focus group discussion methodology: Insights from two decades of application in conservation. Methods in Ecology and Evolution, 9(1), 20-32. https://doi.org/10.1111/2041$\underline{210 X .12860}$

Okada, Y., Sawaumi, T., \& Ito, T. (2017). Effects of observing model video presentations on Japanese EFL learners' oral performance. Electronic Journal of Foreign Language Teaching, 14(2), 129-144.

Onurkan Aliusta, G., \& Özer, B. (2017). Student-centred learning (SCL): Roles changed? Teachers and Teaching, 23(4), 422-435. https://doi.org/10.1080/13540602.2016.1205014

Paliwoda-Pękosz, G., \& Stal, J. (2015). ICT in supporting content and language integrated learning: Experience from Poland. Information Technology for Development: ICT in Transition Economies, 21(3), 403-425. https://doi.org/10.1080/02681102.2014.1003521

Parahoo, K. (2014). Nursing research: Principles, process and issues. Palgrave Macmillan.

Parkes, M., Stein, S., \& Reading, C. (2015a). Student preparedness for university e-learning environments. The Internet and Higher Education, 25(2015), 1-10.

https://doi.org/10.1016/j.iheduc.2014.10.002

Parkes, M., Stein, S., \& Reading, C. (2015b). Student preparedness for university e-learning environments. The Internet and Higher Education, 25(C), 1-10.

https://doi.org/10.1016/j.iheduc.2014.10.002

Patton, M. (2015). Qualitative research \& evaluation methods: Integrating theory and practice: The definitive text of qualitative inquiry frameworks and options. Sage Publications.

Peeraer, J., \& Van Petegem, P. (2011). ICT in teacher education in an emerging developing country: Vietnam's baseline situation at the start of 'The Year of ICT'. Computers \& Education, 56(4), 974-982. https://doi.org/10.1016/i.compedu.2010.11.015 
Peeraer, J., \& Van Petegem, P. (2015). Integration or transformation? Looking in the future of information and communication technology in education in Vietnam. Evaluation and Program Planning, 48, 47-56. https://doi.org/10.1016/j.evalprogplan.2014.09.005

Pellegrino, J. W., Chudowsky, N., \& Glaser, R. (2001). Knowing what students know: The science and design of educational assessment. National Academy Press.

Persson, V., \& Nouri, J. (2018). A systematic review of second language learning with mobile technologies. International Journal Of Emerging Technologies In Learning, 13(2), 188210. https://doi.org/10.3991/ijet.v13i02.8094

Pessoa, S., Miller, R. T., \& Kaufer, D. (2014). Students' challenges and development in the transition to academic writing at an English-medium university in Qatar. International Review of Applied Linguistics in Language Teaching, 52(2), 127-156. https://doi.org/10.1515/iral-2014-0006

Pham, H., \& Fry, G. (2004). Education and economic, political, and social change in Vietnam. Educational Research for Policy and Practice, 3(3), 199-222. https://doi.org/10.1007/s10671-005-0678-0

Pham, T. H. T. (2010). Implementing a student-centered learning approach at Vietnamese higher education institutions: Barriers under. Journal of Futures Studies, 15(1), 21-38.

Pham, T. H. T. (2011). Issues to consider when implementing student-centred learning practices at Asian higher education institutions. Journal of Higher Education Policy and Management, 33(5), 519-528. https://doi.org/10.1080/1360080X.2011.605226

Polit, D. F., \& Beck, C. T. (2008). Nursing research: Generating and assessing evidence for nursing practice. Lippincott Williams \& Wilkins.

Polit, D. F., \& Beck, C. T. (2016). Nursing research : Generating and assessing evidence for nursing practice (10th ed.). Wolters Kluwer Health.

Porter, W. W., \& Graham, C. R. (2016). Institutional drivers and barriers to faculty adoption of blended learning in higher education. British Journal of Educational Technology, 47(4), 748-762. https://doi.org/10.1111/bjet.12269

Price, L., \& Kirkwood, A. (2014). Using technology for teaching and learning in higher education: A critical review of the role of evidence in informing practice. Higher Education Research \& Development, 33(3), 549-564. https://doi.org/10.1080/07294360.2013.841643

Probst, B. (2015). The eye regards itself: Benefits and challenges of reflexivity in qualitative social work research. Social Work Research, 39(1), 37-48.

https://doi.org/10.1093/swr/svu028 
Querol-Julián, M., \& Camiciottoli, B. C. (2019). The impact of online technologies and English medium instruction on university lectures in international learning contexts: A systematic review. ESP Today, 7(1), 2-23. https://doi.org/10.18485/esptoday.2019.7.1.1

Raes, A., Schellens, T., De Wever, B., \& Vanderhoven, E. (2012). Scaffolding information problem solving in web-based collaborative inquiry learning. Computers \& Education, 59(1), 82-

94. https://doi.org/10.1016/j.compedu.2011.11.010

Ranalli, J. (2018). Automated written corrective feedback: How well can students make use of it? Computer Assisted Language Learning, 31(7), 653-674.

https://doi.org/10.1080/09588221.2018.1428994

Richardson, M., Abraham, C., \& Bond, R. (2012). Psychological correlates of university students' academic performance: A systematic review and meta-analysis. Psychological bulletin, 138(2), 353-387. https://doi.org/10.1037/a0026838

Robson, S. (2016). Becoming international: Academic perceptions and practices of internationalising higher education. European Educational Research Association Conference, Dublin.

Rogier, D. (2012). The effects of English-medium instruction on language proficiency of students enrolled in higher education in the UAE (Publication Number U621250) [Doctoral dissertation, University of Exeter]. ProQuest Dissertations \& Theses Global.

Rolfe, G. (2006). Validity, trustworthiness and rigour: Quality and the idea of qualitative research. Journal of advanced nursing, 53(3), 304-310. https://doi.org/10.1111/j.13652648.2006.03727.x

Rose, H., \& McKinley, J. (2018). Japan's English-medium instruction initiatives and the globalization of higher education. Higher Education, 75(1), 111-129.

https://doi.org/10.1007/s10734-017-0125-1

Rosenthal, M. (2016). Qualitative research methods: Why, when, and how to conduct interviews and focus groups in pharmacy research. Currents in pharmacy teaching and learning, 8(4), 509-516. https://doi.org/10.1016/j.cptl.2016.03.021

Ross, E. W., \& Gibson, R. J. (2007). Neoliberalism and education reform. Hampton Press.

Rossman, G. B., \& Rallis, S. F. (2011). Learning in the field: An introduction to qualitative research. Sage Publications.

Rustam, S., \& Mengke, Y. (2020). Review of studies on technology-enhanced language learning and teaching. Sustainability, 12(2), 524-546. https://doi.org/10.3390/su12020524 
Ryan, G. W., \& Bernard, H. R. (2003). Techniques to identify themes. Field methods, 15(1), 85109.

Sahin, I., Celik, I., Oguz Akturk, A., \& Aydin, M. (2013). Analysis of relationships between technological pedagogical content knowledge and educational internet use. Journal of Digital Learning in Teacher Education, 29(4), 110-117. https://doi.org/10.1080/21532974.2013.10784714

Saltan, F., Türkyılmaz, T., Karaçaltı, C., \& Bilir, K. (2018). Use of current educational technology in science education: A scoping review. Çukurova University. Faculty of Education Journal, 47(1), 308-336. https://doi.org/10.14812/cuefd.304015

Saltman, K. J. (2014). The Austerity School. Sympokē, 22(1-2), 41-57. https://doi.org/10.5250/symploke.22.1-2.0041

Sameephet, B. (2020). On the fluidity of languages: A way out of the dilemma in English medium instruction classrooms in Thailand [Doctoral dissertation]. University of Waikato.

San Isidro, X., \& Lasagabaster, D. (2019). Code-switching in a CLIL multilingual setting: A longitudinal qualitative study. International Journal of Multilingualism, 16(3), 336-356. https://doi.org/10.1080/14790718.2018.1477781

Sarhandi, P. S. A., Bajnaid, A., \& Elyas, T. (2017). Impact of Smartphone Based Activities on EFL Students' Engagement. English Language Teaching, 10(6), 103-117.

\section{https://doi.org/10.5539/elt.v10n6p103}

Schleppegrell, M. J. (2020). The knowledge base for language teaching: What is the English to be taught as content? Language Teaching Research, 24(1), 17-27. https://doi.org/10.1177/1362168818777519

Schmid, E. C. (2011). Video-stimulated reflection as a professional development tool in interactive whiteboard research. ReCALL, 23(03), 252-270.

\section{https://doi.org/10.1017/S0958344011000176}

Schmid, R. F., Bernard, R. M., Borokhovski, E., Tamim, R. M., Abrami, P. C., Surkes, M. A., Wade, C. A., \& Woods, J. (2014). The effects of technology use in postsecondary education: A meta-analysis of classroom applications. Computers and education, 72(2014), 271-291. https://doi.org/10.1016/j.compedu.2013.11.002

Schmitt, N., Schmitt, D., \& Clapham, C. (2001). Developing and exploring the behaviour of two new versions of the vocabulary levels test. Language Testing, 18(1), 55-88. https://doi.org/10.1177/026553220101800103 
Selwyn, N. (2010). Looking beyond learning: notes towards the critical study of educational technology. Journal of Computer assisted learning, 26(1), 65-73. https://doi.org/10.1111/j.1365-2729.2009.00338.x

Selwyn, N. (2016). Digital downsides: Exploring university students' negative engagements with digital technology. Teaching in Higher Education, 21(8), 1006-1021. https://doi.org/10.1080/13562517.2016.1213229

Shadiev, R., Hwang, W.-Y., \& Liu, T.-Y. (2018). A study of the use of wearable devices for healthy and enjoyable English as a foreign language learning in authentic contexts. Educational Technology \& Society, 21(4), 217-231.

Shelton, C. (2014). Teacher thinking about technology in higher education: Putting pedagogy and identity in context [Doctoral dissertation]. University College London.

Shen, H., Yuan, Y., \& Ewing, R. (2015). English learning websites and digital resources from the perspective of Chinese university EFL practitioners. 27(2), 156-176.

\section{https://doi.org/10.1017/S0958344014000263}

Shenton, A. K. (2004). Strategies for ensuring trustworthiness in qualitative research projects. Education for information, 22(2), 63-75. https://doi.org/10.3233/EFl-2004-22201

Shim, R. J., \& Baik, M. J. (2003). English education in South Korea. . In H. W. Kam \& R. Y. L. Wong (Eds.), English language teaching in East Asia today: Changing policies and practices (pp. 234-256). Singapore: Eastern Universities Press.

Shulman, L. S. (1986). Those who understand: Knowledge growth in teaching. Educational Researcher, 15(2), 4-14. https://doi.org/10.3102/0013189X015002004

Shulman, L. S. (1987). Knowledge and teaching: Foundations of the new reform. Harvard educational review, 57(1), 1-23.

\section{https://doi.org/https://doi.org/10.17763/haer.57.1.j463w79r56455411}

Shulman, L. S. (2013). Those who understand: Knowledge growth in teaching. Journal of Education, 193(3), 1-11.

Smale, M. A. (2017). Digital technology as affordance and barrier in higher education (1st ed.). Springer International Publishing. https://doi.org/10.1007/978-3-319-48908-7

Smirnova, Z., Vaganova, O., Eltanskaya, E., Lizunkov, V., \& Parsieva, L. (2019). Implementation of a student-centered approach in blended learning in a higher educational institution. SHS Web of Conferences, 69. https://doi.org/10.1051/shsconf/20196900112 
Smit, N., van de Grift, W., de Bot, K., \& Jansen, E. (2017). A classroom observation tool for scaffolding reading comprehension. System, 65(2017), 117-129. https://doi.org/10.1016/i.system.2016.12.014

Smit, U., \& Dafouz, E. (2012). Integrating content and language in higher education: An introduction to English-medium policies, conceptual issues and research practices across Europe. Aila Review, 25(1), 1-12.

Smylie, M. A. (1989). Teachers' views of the effectiveness of sources of learning to teach. The elementary school journal, 89(5), 543-558. https://doi.org/10.1086/461591

Soffer, T., \& Nachmias, R. (2018). Effectiveness of learning in online academic courses compared with face-to-face courses in higher education. Journal of Computer assisted learning, 34(5), 534-543. https://doi.org/10.1111/jcal.12258

Song, Y., \& Looi, C.-K. (2012). Linking teacher beliefs, practices and student inquiry-based learning in a CSCL environment: A tale of two teachers. International Journal of Computer-Supported Collaborative Learning, 7(1), 129-159.

https://doi.org/10.1007/s11412-011-9133-9

Spiteri, M. (2020). Literature review on the factors affecting primary teachers' use of digital technology. Technology, Knowledge and Learning, 25(1), 115-128.

https://doi.org/10.1007/s10758-018-9376-x

Stake, R. E. (1995). The art of case study research. Sage Publications.

Stake, R. E. (2005). Qualitative case studies. In N. K. Denzin \& Y. S. Lincoln (Eds.), The Sage handbook of qualitative research (3rd ed.). Sage Publications

Stake, R. E. (2013). Multiple case study analysis. Guilford press.

Starkey, L. (2010). Teachers' pedagogical reasoning and action in the digital age. Teachers and Teaching: theory and practice, 16(2), 233-244.

https://doi.org/10.1080/13540600903478433

Starkey, L. (2011). Evaluating learning in the 21st century: A digital age learning matrix. Technology, Pedagogy and Education, 20(1), 19-39. https://doi.org/10.1080/1475939X.2011.554021

Starkey, L. (2012). Teaching and learning in the digital age. Routledge.

Sumuer, E. (2018). Factors related to college students' self-directed learning with technology. Australasian Journal of Educational Technology, 34(4), 29-43. 
Sweeney, D. (2017). How university teachers and students use educational technology in university classroom contexts to optimise learning : a study of purposes, principles, processes and perspectives (Publication Number 13912169) [Doctoral dissertation, University of Leicester]. ProQuest Dissertations \& Theses Global.

Symon, M., \& Weinberg, L. (2013). Teaching in English: Perceptions from Israel. Forum Winter, (2013), 22-24.

Tai, H.-Y. (2015). Writing development in syntactic complexity, accuracy and fluency in a content and language integrated learning class. International Journal of Language Linguistics, 2(3), 149-156.

Taimalu, M., \& Luik, P. (2019). The impact of beliefs and knowledge on the integration of technology among teacher educators: A path analysis. Teaching and teacher education, 79(2019), 101-110. https://doi.org/10.1016/j.tate.2018.12.012

Tam, A. C. F. (2015). The role of a professional learning community in teacher change: $A$ perspective from beliefs and practices. Teachers and Teaching, 21(1), 22-43.

\section{https://doi.org/10.1080/13540602.2014.928122}

Tarling, I., \& Ng'ambi, D. (2016). Teachers pedagogical change framework: A diagnostic tool for changing teachers' uses of emerging technologies. British Journal of Educational Technology, 47(3), 554-572. https://doi.org/10.1111/bjet.12454

Tarnopolsky, O. B., \& Goodman, B. A. (2014). The ecology of language in classrooms at a university in eastern Ukraine. Language Education, 28(4), 383-396. https://doi.org/10.1080/09500782.2014.890215

Tatzl, D., \& Messnarz, B. (2013). Testing foreign language impact on engineering students' scientific problem-solving performance. European Journal of Engineering Education, 38(6), 620-630. https://doi.org/10.1080/03043797.2012.719001

Taylor, L., \& Clark, S. (2010). Educational Design of Short, Audio-Only Podcasts: The Teacher and Student Experience. Australasian Journal of Educational Technology, 26(3), 386. https://doi.org/10.14742/ajet.1082

Taylor, M. W. (2013). Replacing the 'teacher-proof'curriculum with the 'curriculumproof'teacher: Toward more effective interactions with mathematics textbooks. Journal of curriculum studies, 45(3), 295-321. https://doi.org/10.1080/00220272.2012.710253

Teo, T. (2010). A path analysis of pre-service teachers' attitudes to computer use: Applying and extending the technology acceptance model in an educational context. Interactive Learning Environments, 18(1), 65-79. https://doi.org/10.1080/10494820802231327 
Teo, T., Sang, G., Mei, B., \& Hoi, C. K. W. (2019). Investigating pre-service teachers' acceptance of Web 2.0 technologies in their future teaching: A Chinese perspective. Interactive Learning Environments, 27(4), 530-546.

\section{https://doi.org/10.1080/10494820.2018.1489290}

Then, D. C.-O., \& Ting, S.-H. (2011). Code-switching in English and science classrooms: More than translation. International Journal of Multilingualism, 8(4), 299-323. https://doi.org/10.1080/14790718.2011.577777

Thøgersen, J., \& Airey, J. (2011). Lecturing undergraduate science in Danish and in English: A comparison of speaking rate and rhetorical style. English for Specific Purposes, 30(3), 209-221. https://doi.org/10.1016/j.esp.2011.01.002

Thorpe, M. (2002). From independent learning to collaborative learning. Distributed learning: Social cultural approaches to practice, 131.

Timmermans, S., \& Tavory, I. (2012). Theory construction in qualitative research: From grounded theory to abductive analysis. Sociological Theory, 30(3), 167-186.

\section{https://doi.org/10.1177/0735275112457914}

Tobin, G. A., \& Begley, C. M. (2004). Methodological rigour within a qualitative framework. Journal of advanced nursing, 48(4), 388-396. https://doi.org/10.1111/j.1365$\underline{2648.2004 .03207 . x}$

Tomlinson, C., \& Moon, T. (2013). Differentiation and classroom assessment. In J. H. McMillan (Ed.), SAGE handbook of research on classroom assessment (pp. 415-430). Sage Publications.

Tondeur, J., Braak, J., Ertmer, P., \& Ottenbreit-Leftwich, A. (2017). Understanding the relationship between teachers' pedagogical beliefs and technology use in education: $A$ systematic review of qualitative evidence. Educational Technology Research and Development, 65(3), 555-575. https://doi.org/10.1007/s11423-016-9481-2

Toom, A., Pyhältö, K., \& Rust, F. O. C. (2015). Teachers' professional agency in contradictory times. Teachers and Teaching, 21(6), 615-623.

\section{https://doi.org/10.1080/13540602.2015.1044334}

Tour, E. (2015). Digital mindsets: Teachers' technology use in personal life and teaching. Language Learning \& Technology, 19(3), 124-139. https://doi.org/10125/44437

Tran, L. H. N., Phan, T. N. P., \& Tran, H. L. K. (2018). Implementing the student-centred teaching approach in Vietnamese universities: The influence of leadership and management 
practices on teacher engagement. Educational Studies, 46(2), 1-17.

https://doi.org/10.1080/03055698.2018.1555453

Tran, L. T., Le, T. T. T., \& Nguyen, N. T. (2014). Curriculum and pedagogy. In N. Clark (Ed.), Higher education in Vietnam (pp. 86-107). Springer.

Tran, L. T., \& Nguyen, H. T. (2018). Internationalisation of higher education in Vietnam through English-medium instruction (EMI): Practices, tensions and implications for local language policies. In I. Liyanage (Ed.), Multilingual Education Yearbook 2018 (pp. 91-106). Springer. https://doi.org/https://doi.org/10.1007/978-3-319-77655-2 6

Tran, T. D. (1995). Ve anh huong cua Nho giao doi voi con nguoi Viet Nam trong lich su. Triet hoc, 4, 58-61.

Tran, V. D., \& Lewis, R. (2012). The effects of jigsaw learning on students' attitudes in a Vietnamese higher education classroom. International Journal of Higher Education, 1(2), 9-20. https://doi.org/10.5430/ijhe.v1n2p9

Trinidad, J. E., \& Ngo, G. R. (2019). Technology's roles in student-centred learning in higher education. International Journal of Action Research, 15(1), 81-94.

Tripp, T. R., \& Rich, P. J. (2012). The influence of video analysis on the process of teacher change. Teaching and teacher education, 28(5), 728-739.

https://doi.org/10.1016/j.tate.2012.01.011

Truong, T. D., Hallinger, P., \& Sanga, K. (2017). Confucian values and school leadership in Vietnam: Exploring the influence of culture on principal decision making. Educational Management Administration \& Leadership, 45(1), 77-100. https://doi.org/10.1177/1741143215607877

Tsai, Y.-R. (2019). Promotion of learner autonomy within the framework of a flipped EFL instructional model: Perception and perspectives. Computer Assisted Language Learning(2019), 1-32. https://doi.org/10.1080/09588221.2019.1650779

Tschannen-Moran, M. (2001). Collaboration and the need fortrust. Journal of Educational administration, 39(4), 308-331. https://doi.org/10.1108/EUM0000000005493

Turan, Z., \& Akdag-Cimen, B. (2019). Flipped classroom in English language teaching: A systematic review. Computer Assisted Language Learning. https://doi.org/10.1080/09588221.2019.1584117

Türk, E., \& Erçetin, G. (2014). Effects of interactive versus simultaneous display of multimedia glosses on $\mathrm{L} 2$ reading comprehension and incidental vocabulary learning. Computer 
Assisted Language Learning, 27(1), 1-25.

https://doi.org/10.1080/09588221.2012.692384

Turkan, S., De Oliveira, L. C., Lee, O., \& Phelps, G. (2014). Proposing a knowledge base for teaching academic content to English language learners: Disciplinary linguistic knowledge. Teachers College Record, 116(3), 1-30.

Tursunov, M. (2016). A comparative analysis of teacher-centered and learner-centered classes. Міжнародний науковий журнал, 6(3), 65-67.

Tynjälä, P., \& Gijbels, D. (2012). Changing world: Changing pedagogy. In P. Tynjala, M.-L. Stenström, \& M. Saarnivaara (Eds.), Transitions and transformations in learning and education (pp. 205-222). Springer.

Uchihara, T., \& Harada, T. (2018). Roles of vocabulary knowledge for success in English-medium instruction: Self-perceptions and academic outcomes of Japanese undergraduates. TESOL Quarterly, 52(3), 564-587. https://doi.org/10.1002/tesq.453

Vähäsantanen, K. (2013). Vocational teachers' professional agency in the stream of change [Unpublished dotoral thesis]. University of Jyväskylä.

Vähäsantanen, K. (2015). Professional agency in the stream of change: Understanding educational change and teachers' professional identities. Teaching and teacher education, 47(2015), 1-12. https://doi.org/10.1016/j.tate.2014.11.006

Vaismoradi, M., Jones, J., Turunen, H., \& Snelgrove, S. (2016). Theme development in qualitative content analysis and thematic analysis. 6(5), 100-110.

https://doi.org/10.5430/jnep.v6n5p100

Valtonen, T., Kukkonen, J., Kontkanen, S., Sormunen, K., Dillon, P., \& Sointu, E. (2015). The impact of authentic learning experiences with ICT on pre-service teachers' intentions to use ICT for teaching and learning. Computers \& Education, 81(2015), 49-58. https://doi.org/10.1016/i.compedu.2014.09.008

Valtonena, T., Kukkonena, J., \& Wulffb, A. (2006). High school teachers' course designs and their professional knowledge of online teaching. Informatics in Education, 5(2), 313-328.

Van Lier, L. (2004). The semiotics and ecology of language learning. Utbildning \& Demokrati, 13(3), 79-103.

Van Lier, L. (2008). Agency in the classroom. In J. P. Lantolf \& M. E. Poehner (Eds.), Sociocultural theory and the teaching of second languages (pp. 1163-1186). Equinox Publications. 
Vasodavan, V., DeWitt, D., \& Alias, N. (2019). TPACK In higher education: Analysis of the collaborative tools used by lecturers. JuKu: Jurnal Kurikulum \& Pengajaran Asia Pasifik, 7(1), 9-17.

Venkatesh, V., Morris, M. G., Davis, G. B., \& Davis, F. D. (2003). User acceptance of information technology: Toward a unified view. MIS Quarterly, 27(3), 425-478.

https://doi.org/10.2307/30036540

Vietnamese Government. (2008). Decision No. 1400/QD-TTg on "Teaching and learning foreign languages in the national education system, period 2008-2020".

http://www.chinhphu.vn/portal/page/portal/chinhphu/hethongvanban?class_id=1\&

Vietnamese Government. (2012). Chiến lược phát triển giáo dục 2011-2020 (Strategy for education development 2011-2020).

http://vanban.chinhphu.vn/portal/page/portal/chinhphu/hethongvanban?class id=2\& $\underline{\text { mode }=\text { detail } \& \text { document id }=160806}$

Voogt, J., Fisser, P., Pareja Roblin, N., Tondeur, J., \& Van Braak, J. (2013). Technological pedagogical content knowledge: A review of the literature. Journal of Computer assisted learning, 29(2), 109-121. https://doi.org/10.1111/j.1365-2729.2012.00487.x

Vu, N. T., \& Burns, A. (2014). English as a medium of instruction: Challenges for Vietnamese tertiary lecturers. The journal of Asia TEFL, 11(3), 1-31.

Walker, R., Jenkins, M., \& Voce, J. (2018). The rhetoric and reality of technology-enhanced learning developments in UK higher education: Reflections on recent UCISA research findings (2012-2016). Interactive Learning Environments, 26(7), 858-868. https://doi.org/10.1080/10494820.2017.1419497

Walkinshaw, I., Fenton-Smith, B., \& Humphreys, P. (2017). EMI issues and challenges in AsiaPacific higher education: An introduction. In B. Fenton-Smith, P. Humphreys, \& I. Walkinshaw (Eds.), English Medium Instruction in Higher Education in Asia-Pacific (Vol. 21, pp. 1-18). Springer. https://doi.org/10.1007/978-3-319-51976-0

Walqui, A., \& Van Lier, L. (2010). Scaffolding the academic success of adolescent English language learners: A pedagogy of promise. WestEd.

Wang, W., \& Curdt-Christiansen, X. L. (2019). Translanguaging in a Chinese-English bilingual education programme: A university-classroom ethnography. International Journal of Bilingual Education and Bilingualism, 22(3), 322-337. 
Washington, G. Y. (2019). The learning management system matters in face-to-face higher education courses. Journal of Educational Technology Systems, 48(2), 255-275. https://doi.org/10.1177/0047239519874037

Wass, R., Harland, T., \& Mercer, A. (2011). Scaffolding critical thinking in the zone of proximal development. Higher Education Research \& Development, 30(3), 317-328. https://doi.org/10.1080/07294360.2010.489237

Weis, L., \& Fine, M. (2012). Critical bifocality and circuits of privilege: Expanding critical ethnographic theory and design. Harvard educational review, 82(2), 173-201. https://doi.org/10.17763/haer.82.2.v1jx34n441532242

Werther, C., Denver, L., Jensen, C., \& Mees, I. M. (2014). Using English as a medium of instruction at university level in Denmark: The lecturer's perspective. Journal of Multilingual Multicultural Development, 35(5), 443-462. https://doi.org/10.1080/01434632.2013.868901

Wihlborg, M., \& Robson, S. (2018). Internationalisation of higher education: Drivers, rationales, priorities, values and impacts. European Journal of Higher Education, 8(1), 8-18. https://doi.org/10.1080/21568235.2017.1376696

Wilkins, J. (2008). The relationship among elementary teachers' content knowledge, attitudes, beliefs, and practices. Journal of Mathematics Teacher Education, 11(2), 139-164.

\section{https://doi.org/10.1007/s10857-007-9068-2}

Wilkinson, R. (2011). If all business education were in English, would it matter? ITL International Journal of Applied Linguistics, 161(1), 111-123. https://doi.org/10.1075/itl.161.07wil

Williams, E. N., \& Morrow, S. L. (2009). Achieving trustworthiness in qualitative research: A panparadigmatic perspective. Psychotherapy Research, 19(4-5), 576-582.

\section{https://doi.org/10.1080/10503300802702113}

Winstone, N., \& Bretton, H. (2013). Strengthening the transition to university by confronting the expectation-reality gap in psychology undergraduates. Psychology Teaching Review, 19(2), 2-14.

Won, E.-S., \& Kim, J.-R. (2018). The effectiveness of self-directed English learning through SNS: Adopting Facebook based on gamification. International Journal of Mobile and Blended Learning (IJMBL), 10(3), 1-10. https://doi.org/10.4018/IJMBL.2018070101 
Wood, D., Bruner, J. S., \& Ross, G. (1976). The role of tutoring in problem solving. Journal of child psychology psychiatry, 17(2), 89-100. https://doi.org/10.1111/j.14697610.1976.tb00381.x

Wu, J. G. (2018). Mobile collaborative learning in a Chinese tertiary EFL context. TESL-EJ, 22(2), $1-15$.

Wyatt, H. T., Krauskopf, B. P., Gaylord, M. N., Ward, M. A., Huffstutler-Hawkins, M. S., \& Goodwin, M. L. (2010). Cooperative M-Learning with nurse practitioner students. Nursing Education Perspective, 31(2), 109-113.

Yang, J., Wang, Q., Wang, J., Huang, M., \& Ma, Y. (2019). A study of K-12 teachers' TPACK on the technology acceptance of E-schoolbag. Interactive Learning Environments(2019), 1-15. https://doi.org/10.1080/10494820.2019.1627560

Yang, W. (2015). Content and language integrated learning next in Asia: Evidence of learners' achievement in CLIL education from a Taiwan tertiary degree programme. International Journal of Bilingual Education Bilingualism, 18(4), 361-382.

\section{https://doi.org/10.1080/13670050.2014.904840}

Yeh, C.-C. (2014). Taiwanese students' experiences and attitudes towards English-medium courses in tertiary education. Relc Journal, 45(3), 305-319.

\section{https://doi.org/10.1177/0033688214555358}

Yin, R. K. (2013). Case study research: Design and methods. Sage publications.

Yin, R. K. (2018). Case study research and applications: Design and methods (6th ed.). Sage Publications.

Yusop, F., \& Razak, R. (2020). The role of TPACK in affecting pre-service language teachers' ICT integration during teaching practices: Indonesian context. Education and Information Technologies, 25(3), 1929-1949. https://doi.org/10.1007/s10639-019-10040-2

Zainuddin, Z., \& Perera, C. J. (2017). Exploring students' competence, autonomy and relatedness in the flipped classroom pedagogical model. Journal of Further and Higher Education, 43(1), 1-12. https://doi.org/10.1080/0309877X.2017.1356916

Zajda, J., \& Rust, V. (2016). Globalisation and Higher Education Reforms (Vol. 15). Springer International Publishing, Cham. https://doi.org/10.1007/978-3-319-28191-9

Zhonggen, Y. (2018). Differences in serious game-aided and traditional English vocabulary acquisition. Computers \& Education, 127(2018), 214-232.

https://doi.org/10.1016/j.compedu.2018.07.014 
Ziegler, N. (2016). Synchronous computer-mediated communication and interaction: A metaanalysis. Studies in Second Language Acquisition, 38(3), 553-586.

https://doi.org/10.1017/S027226311500025X 


\section{Appendices}

\section{Appendix A. Information sheet}

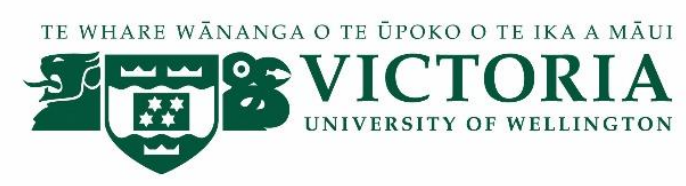

Dear

Project title:

\section{DIGITAL TECHNOLOGIES IN AN ENGLISH-MEDIUM INSTRUCTIONN CONTEXT}

A CASE STUDY OF VIETNAMESE HIGHER EDUCATION TEACHERS AND STUDENTS

Thank you for your interest in this project. Please read this information before deciding whether or not to take part. If you decide to participate, thank you. If you decide not to take part, thank you for considering my request.

\section{Who am I?}

My name is Tho Vo and I am a lecturer at the School of Foreign Languages for Economics, University of Economics Ho Chi Minh City. I am currently a Doctoral student in Education at Victoria University of Wellington. This research project is work towards my thesis.

\section{What is the aim of the project?}

This project aims to explore the use of digital technologies in a context where several subjects are learnt and taught in English (using a content and language integrated learning approach - CLIL approach) at tertiary level in a Vietnamese university. First, I would like to examine how both teachers and students perceive and adapt to the emerging context of CLIL. Then I will investigate the ways that digital technologies are used for learning and teaching in this context. This research has been approved by the Victoria University of Wellington Human Ethics Committee [provide approval number].

I am seeking your permission to conduct research within the University of Economics Ho Chi Minh City. If you agree to take part in, I will select six subject teachers to involve in my research as case studies. Each teacher will be invited to participate in two interviews. I will ask them about their perceptions and experience of using digital technologies in contexts where they have to teach their subjects in English. Six groups of students will be invited to focus-group interviews. I will ask them about their use of digital technologies in learning activities in content and language integrated learning context. Each interview will take $30-45$ minutes. All interviews will be audio-recorded. One classroom observation will be conducted with each teachers and his/her class. The teachers will select a class in which they conduct one 3-hour lesson for observations. All observations will be 
video recorded. You can withdraw from the study by contacting me at any point before $28^{\text {th }}$ February 2018.

\section{What will happen to the information you give?}

Throughout the project all attempts will be made to minimize the disruptive impact on teaching and learning activities of your teachers and students. Once the study is complete, your university will be provided with a summary of the research findings and any publications that result from the study. This research is confidential. This means that the researchers named below will be aware of your identity, but the research data will be aggregated, and your identity will not be disclosed in any reports, presentations, or public documentation. Your university will not be identified in any work generated from this study. Only my supervisors and I will read the notes or transcript of the interview. The interview transcripts, summaries and any recordings will be kept securely and destroyed 2 years after the research ends.

\section{What will the project produce?}

The information from my research will be used in my PhD dissertation.

\section{If you accept this invitation, what are your rights as a research participant?}

You do not have to accept this invitation if you don't want to. If you do decide to participate, you have the right to:

- $\quad$ withdraw from the study before $28^{\text {th }}$ February 2018;

- $\quad$ ask any questions about the study at any time;

- be able to read any reports or a full version of this research by emailing the researcher to request a copy.

If you have any questions, either now or in the future, please feel free to contact either:

\section{Student:}

Name: Tho Vo

University email address:

Tho.Vo@vuw.ac.nz

\section{Supervisor:}

Name: Dr. Louise Starkey -Dr. Margaret Gleeson

Role: Lecturers

School: Education

Phone:

Louise.Starkey@vuw.ac.nz

Margaret.Gleeson@vuw.ac.nz

\section{Human Ethics Committee information}

If you have any concerns about the ethical conduct of the research, you may contact the Victoria University HEC Convener: Associate Professor Susan Corbett. Email susan.corbett@vuw.ac.nz or telephone +64-4-463 5480 . 


\section{Appendix B. Consent form}

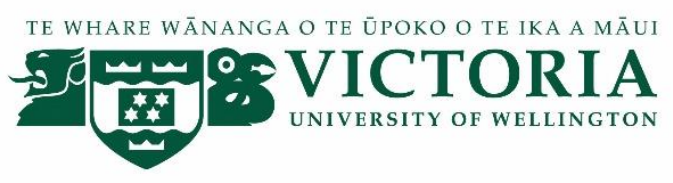

\section{DIGITAL TECHNOLOGIES IN AN ENGLISH-MEDIUM INSTRUCTION CONTEXT}

A case study of Vietnamese higher education teachers and students

\section{CONSENT TO GETTING ACCESS TO TEACHERS AND STUDENTS}

This consent form will be held for 4 years.

Researcher: Tho Vo, School of Education, Victoria University of Wellington.

* I have read the Information Sheet and the project has been explained to me. My questions have been answered to my satisfaction. I understand that I can ask further questions at any time.

* I permit the researcher to get access to teachers and students at the university to invite them to participate in his study.

I understand that:

* I may withdraw from this study at any point before $28^{\text {th }}$ February 2018 , without giving any reason.

* Any information I provide will be kept confidential to the researcher and his supervisor. I understand that the results will be used for a PhD report and a summary of the results may be used in academic reports and/or presented at conferences.

* My university's name will not be used in reports, nor will any information that would identify my university.

* Teachers and students at my university participating in the study must be guaranteed to be under ethics consideration.

* I would like to receive a full copy of the thesis and have added my email address below.

Signature of participant:

Name of participant:

Date:

Contact details: 
Appendix C. Observation checklist for field-notes

TE WHARE WĀNANGA O TE ÜPOKO O TE IKA A MĀUI

ST VICTORIA

* * $\propto$ University of Wellington

OBSERVATION CHECKLIST FOR FIELD-NOTES

\begin{tabular}{|l|l|l|l|l|l|}
\hline PREPARATION & & SCAFFOLDING & & GROUPING OPTIONS & \\
\hline Adaptation of content & 1 & Modelling & 5 & Whole class & 9 \\
\hline Links to background & 2 & Guided practice & 6 & Small groups & 10 \\
\hline Links to past learning & 3 & Independent practice & 7 & Partners & 11 \\
\hline Strategies incorporated & 4 & Comprehensible input & 8 & Independent & 12 \\
\hline PROCESSES & & APPLICATION & & ASSESSMENT & \\
\hline Reading & 13 & Hands-on & 17 & Individual & 21 \\
\hline Writing & 14 & Meaningful & 18 & Group & 22 \\
\hline Speaking & 15 & Links to objectives & 19 & Written & 23 \\
\hline Listening & 16 & Promotes engagement & 20 & Oral & 24 \\
\hline
\end{tabular}

\begin{tabular}{|l|l|l|l|}
\hline Activities & Time & Use of language & Use of digital technologies \\
\hline & & & \\
\hline & & & \\
\hline & & & \\
\hline & & & \\
\hline & & & \\
\hline
\end{tabular}


Appendix D. Students' information worksheet and focus group rules

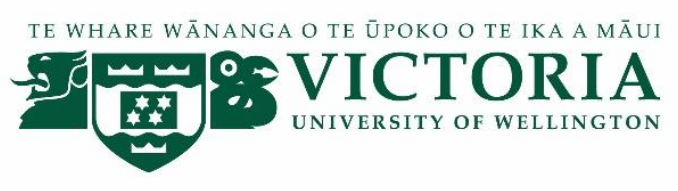

STUDENTS' INFORMATION WORKSHEET

PERSONAL INFORMATION

\begin{tabular}{|l|l|}
\hline Name & \\
\hline Class & \\
\hline Major & \\
\hline Email & \\
\hline Phone number & \\
\hline
\end{tabular}

\section{FIELD OF CONFIDENCE}

\begin{tabular}{|l|l|l|}
\hline 1 & Subject content & \\
\hline 2 & Literacy of digital technologies & \\
\hline 3 & Language competence & \\
\hline
\end{tabular}

\begin{tabular}{|c|c|c|c|c|c|c|}
\hline 1 & $\begin{array}{l}\text { How often do you } \\
\text { use digital } \\
\text { technologies on } \\
\text { daily basis? }\end{array}$ & Never & Rarely & Sometimes & Often & Always \\
\hline 2 & $\begin{array}{l}\text { How often do you } \\
\text { use digital } \\
\text { technologies in } \\
\text { learning? }\end{array}$ & Never & Rarely & Sometimes & Often & Always \\
\hline \multirow{8}{*}{3} & \multirow{8}{*}{$\begin{array}{l}\text { How do you feel } \\
\text { about using digital } \\
\text { technologies in } \\
\text { learning? }\end{array}$} & $\begin{array}{c}\text { Very } \\
\text { unhelpful }\end{array}$ & & & & $\begin{array}{l}\text { Very } \\
\text { helpful }\end{array}$ \\
\hline & & 1 & 2 & 3 & 4 & 5 \\
\hline & & $\begin{array}{c}\text { Very } \\
\text { uninteresting }\end{array}$ & & & & $\begin{array}{c}\text { Very } \\
\text { interesting }\end{array}$ \\
\hline & & 1 & 2 & 3 & 4 & 5 \\
\hline & & $\begin{array}{c}\text { Very } \\
\text { inconvenient }\end{array}$ & & & & $\begin{array}{c}\text { Very } \\
\text { convenient }\end{array}$ \\
\hline & & 1 & 2 & 3 & 4 & 5 \\
\hline & & Very easy & & & & $\begin{array}{c}\text { Very } \\
\text { difficult }\end{array}$ \\
\hline & & 1 & 2 & 3 & 4 & 5 \\
\hline
\end{tabular}

\section{INTEREST IN PARTICIPATING IN FOCUS GROUP INTERVIEWS}

Are you willing to participate in a focus group interview?

Yes $\square$ No $\square$ 


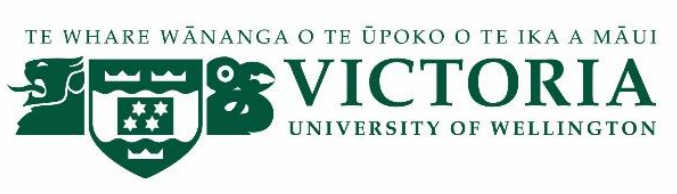

\section{GROUND RULES FOR FOCUS GROUPS}

\section{WELCOME}

Thanks for agreeing to be part of the focus group. We appreciate your willingness to participate.

\section{PURPOSE OF FOCUS GROUPS}

The reason we are having these focus groups is to find out how you use digital technologies in your learning activities and what experience you have when studying in a content and language integrated learning setting.

We need your input and want you to share your honest and open thoughts with us.

\section{GROUND RULES}

1. WE WANT YOU TO DO THE TALKING.

Let us hear from everyone!

One person at a time.

I may call on you if I have not heard from you in a while.

2. THERE ARE NO RIGHT OR WRONG ANSWERS.

Everyone's ideas and experiences are valuable.

It is important to hear all sides - including both positives and negatives.

We will not always agree, but we must always show respect for one another.

3. WHAT IS SHARED IN THIS ROOM STAYS IN THIS ROOM.

We will be recording this session so we do not miss anything.

Please keep everything you hear today confidential.

We will summarize themes without identifying individuals by name. 


\section{Appendix E. Questions and prompts for interviews}

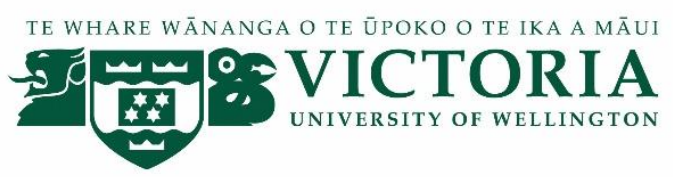

\section{QUESTIONS FOR INTERVIEWS}

First teacher interviews

1. Can you tell me about your experience of teaching in English?

2. Why did you volunteer to teach your subject in English?

3. What challenges and opportunities do you face when teaching business in English?

4. Tell me more about the process of preparing the lessons you will teach.

5. Which digital technologies do you use in your teaching?

6. Why do you use the digital technologies?

7. How do these digital technologies assist in student learning?

8. How do you expect students to use digital technologies?

9. What do you want students to learn from the lesson I will observe?

Second teacher interview

1. Please watch these extracts and tell me why you chose those ways to explain economics concepts to students?

2. Please watch these extracts and tell me why you used those digital technologies?

3. How successful do you think the lesson was?

4. What do you think students learnt well from the lesson? How do you know that?

5. What might you do differently?

6. How did teaching this lesson in English change your previous approach to teaching this topic?

\section{Student focus-group interviews}

1. What did you learn from the previous lesson?

2. What digital technologies do you use for learning?

3. How do you use digital technologies? / Why?

4. Which language(s) do you use when doing such things?

5. What do you do to help you to understand business concepts?

6. How does teaching in English affect your learning? 